\title{
Dirigent Proteins Guide Asymmetric Heterocoupling for the Synthesis of Complex Natural Product Analogues
}

\author{
Stacie S. Kim and Elizabeth S. Sattely*
}

Department of Chemical Engineering and Howard Hughes Medical Institute, Stanford University, Stanford, California, 94305, United States

${ }^{*}$ Correspondence should be addressed to E.S.S. (sattely@stanford.edu)

\section{Table of Contents}

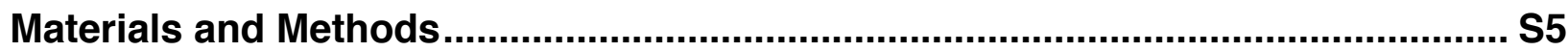

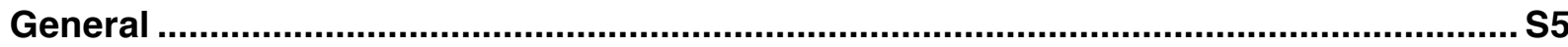

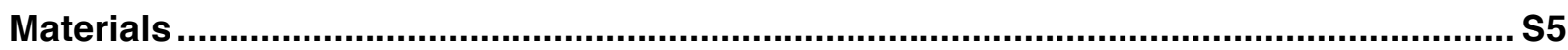

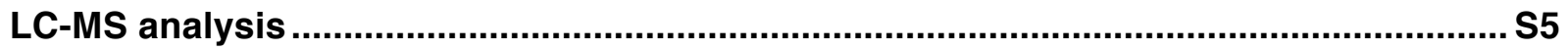

Preparative HPLC purification............................................................................... 6

Crude plant extraction for PhPLR .............................................................................. S6

In-vitro LC-MS end-point analysis of combined PhDIR-PhPLR activity..........................S6

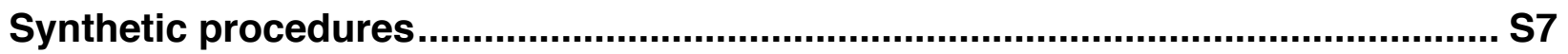

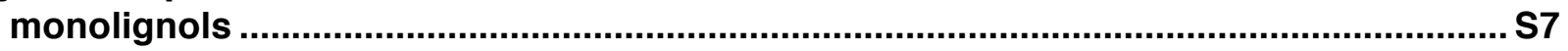

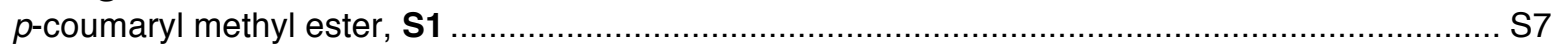

methyl (E)-3-(4-hydroxy-3-(trifluoromethoxy)phenyl)acrylate, S2 …………………………......S7

methyl (E)-3-(3-fluoro-4-hydroxyphenyl)acrylate, S3 …………………………………….... S7

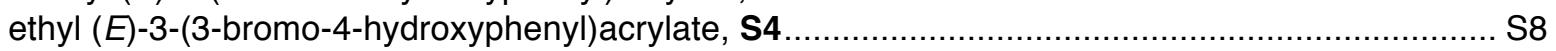

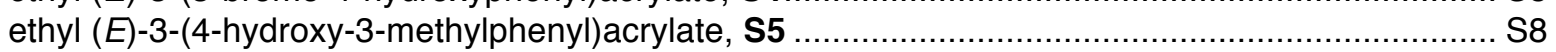

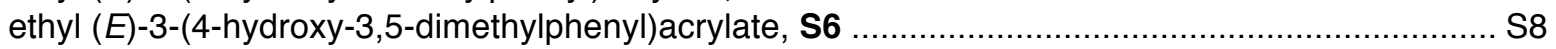

ethyl (E)-3-(3-fluoro-4-hydroxy-5-methoxyphenyl)acrylate, S7 ……………………………….... S9

ethyl (E)-3-(3-ethoxy-4-hydroxyphenyl)acrylate, S8 ……….................................................. S9

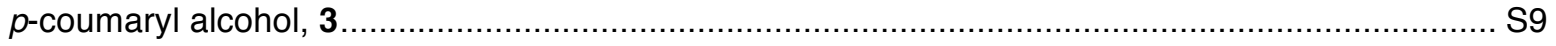

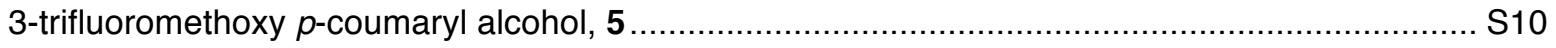

3-fluoro $p$-coumaryl alcohol, 6 .................................................................................... $\$ 10$

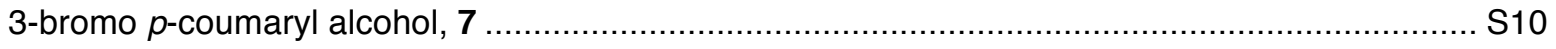

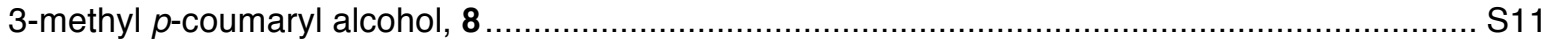

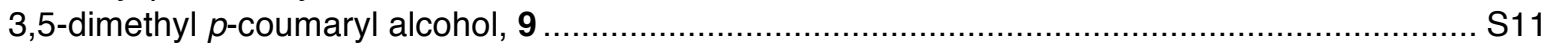

3-fluoro coniferyl alcohol, 10 ..................................................................................... S11

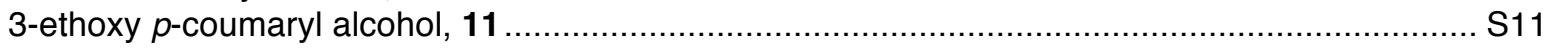

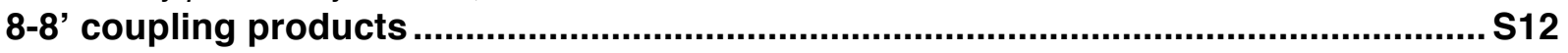

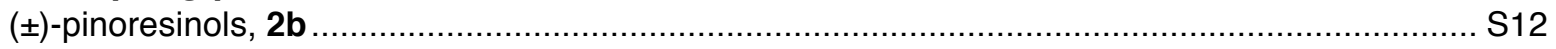

( \pm -dehydrodiconiferyl alcohols, 2c ........................................................................... 512

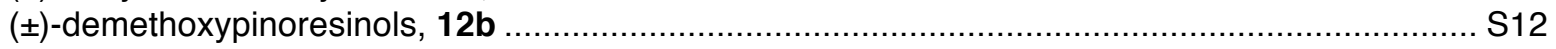

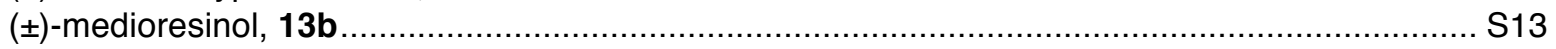

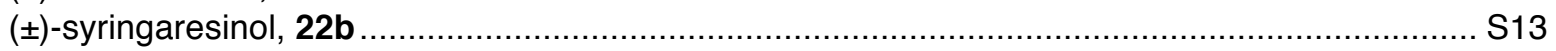

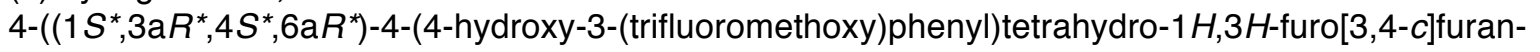

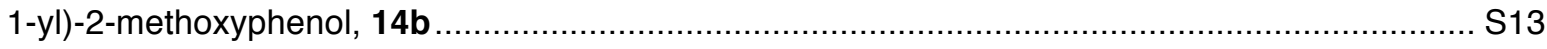


2-fluoro-4-((1 $\left.S^{\star}, 3 a R^{*}, 4 S^{\star}, 6 a R^{*}\right)$-4-(4-hydroxy-3-methoxyphenyl)tetrahydro-1 $H, 3 H$-furo[3,4-c]furan-1yl)phenol, 15b S14 2-bromo-4-((1S*,3a $\left.R^{*}, 4 S^{*}, 6 a R^{*}\right)$-4-(4-hydroxy-3-methoxyphenyl)tetrahydro-1 $H, 3 H$-furo[3,4-c]furan1-yl)phenol, 16b S14 4-((1S*,3a $\left.R^{\star}, 4 S^{\star}, 6 \mathrm{a} R^{\star}\right)-4-(4-h y d r o x y-3-m e t h o x y p h e n y l) t e t r a h y d r o-1 H, 3 H$-furo[3,4-c]furan-1-yl)-2-

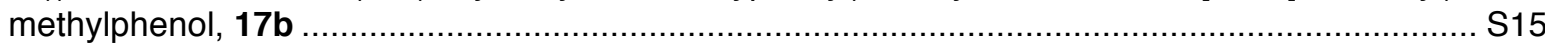
4,4'-((1S $\left.S^{\star}, 3 \mathrm{a} R^{\star}, 4 S^{*}, 6 \mathrm{a} R^{*}\right)$-tetrahydro-1H,3H-furo[3,4-c]furan-1,4-diyl)bis(2-methylphenol), 26b... S15 (E)-4-(3-(hydroxymethyl)-5-(3-hydroxyprop-1-en-1-yl)-7-methoxy-2,3-dihydrobenzofuran-2-yl)-2methylphenol, 17c S15

(E)-4-(3-(hydroxymethyl)-5-(3-hydroxyprop-1-en-1-yl)-7-methyl-2,3-dihydrobenzofuran-2-yl)-2-

methoxyphenol, 17c' .... S15

(E)-4-(3-(hydroxymethyl)-5-(3-hydroxyprop-1-en-1-yl)-7-methyl-2,3-dihydrobenzofuran-2-yl)-2methylphenol, 26c $S 15$ 4-((1 $\left.S^{\star}, 3 \mathrm{a} R^{\star}, 4 S^{\star}, 6 \mathrm{a} R^{*}\right)$-4-(4-hydroxy-3-methoxyphenyl)tetrahydro-1H,3H-furo[3,4-c]furan-1-yl)-2,6dimethylphenol, 18b S16

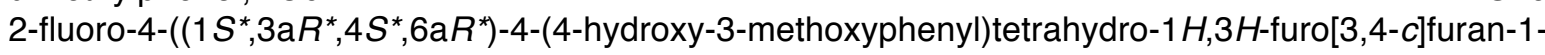
yl)-6-methoxyphenol, 19b S16

(E)-2-fluoro-4-(3-(hydroxymethyl)-5-(3-hydroxyprop-1-en-1-yl)-7-methoxy-2,3-dihydrobenzofuran-2-

yl)-6-methoxyphenol, 19c S16 4,4'-((1 $\left.S^{\star}, 3 \mathrm{a} R^{\star}, 4 S^{\star}, 6 \mathrm{a} R^{\star}\right)$-tetrahydro-1H,3H-furo[3,4-c]furan-1,4-diyl)bis(2-fluoro-6-methoxyphenol),

28b S16

2-ethoxy-4-((1 $\left.S^{\star}, 3 a R^{\star}, 4 S^{*}, 6 a R^{\star}\right)$-4-(4-hydroxy-3-methoxyphenyl)tetrahydro-1 $H, 3 H$-furo[3,4- $c$ ]furan-

1-yl)phenol, 20b S17

4,4'-((1 $\left.S^{*}, 3 \mathrm{a} R^{*}, 4 S^{*}, 6 \mathrm{a} R^{*}\right)$-tetrahydro-1H,3H-furo[3,4-c]furan-1,4-diyl)bis(2-ethoxyphenol), 29b... S17 (E)-2-ethoxy-4-(3-(hydroxymethyl)-5-(3-hydroxyprop-1-en-1-yl)-7-methoxy-2,3-dihydrobenzofuran-2yl)phenol, 20c S17

(E)-4-(7-ethoxy-3-(hydroxymethyl)-5-(3-hydroxyprop-1-en-1-yl)-2,3-dihydrobenzofuran-2-yl)-2methoxyphenol, 20c'

4,4'-((1 $\left.S^{\star}, 3 \mathrm{a} R^{\star}, 4 S^{\star}, 6 \mathrm{a} R^{\star}\right)$-tetrahydro-1H,3H-furo[3,4-c]furan-1,4-diyl)bis(2-ethoxyphenol), 29b... S17 (E)-2-ethoxy-4-(7-ethoxy-3-(hydroxymethyl)-5-(3-hydroxyprop-1-en-1-yl)-2,3-dihydrobenzofuran-2-

yl)phenol, 29c S17

$(+)$-pinoresinol, (+)-2b S18

2-fluoro-4-((1S,3aR,4S,6aR)-4-(4-hydroxy-3-methoxyphenyl)tetrahydro-1H,3H-furo[3,4-c]furan-1-yl)6-methoxyphenol, (+)-19b S18 4,4'-((1S,3aR,4S,6aR)-tetrahydro-1H,3H-furo[3,4-c]furan-1,4-diyl)bis(2-fluoro-6-methoxyphenol), (+)-

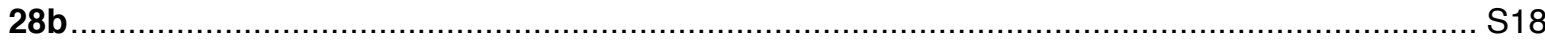
4-((1S,3a R,4S,6a R)-4-(4-hydroxy-3-methoxyphenyl)tetrahydro-1H,3H-furo[3,4-c]furan-1-yl)-2methylphenol, (+)-17b S19 2-ethoxy-4-((1S,3aR,4S,6aR)-4-(4-hydroxy-3-methoxyphenyl)tetrahydro-1H,3H-furo[3,4-c]furan-1-

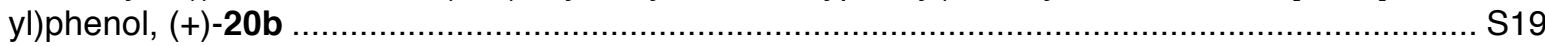
4,4'-((1S,3aR,4S,6aR)-tetrahydro-1H,3H-furo[3,4-c]furan-1,4-diyl)bis(2-ethoxyphenol), (+)-29b .. S19

\section{Supplementary Figures and Table.} S21

Figure S1. Chemical structure naming convention for all coupling products .......................... S21 Figure S2. PhDIR mediates regioselective coupling of 1 and heterocoupling of 1 with 3, 4, 5, 7 and 9.

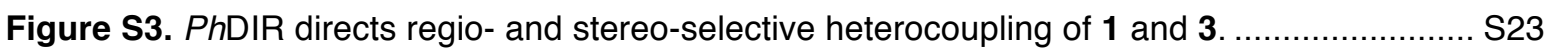
Figure S4. PhDIR directs regio- and stereo-selective heterocoupling of 1 and 4 ..................... S24

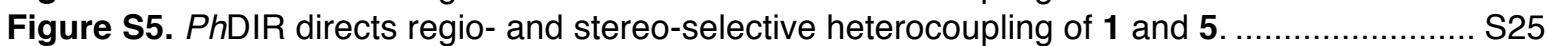

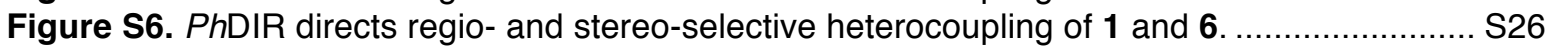

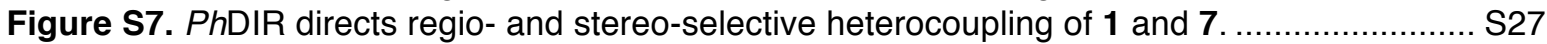

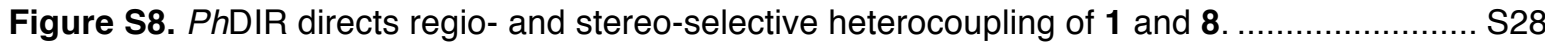

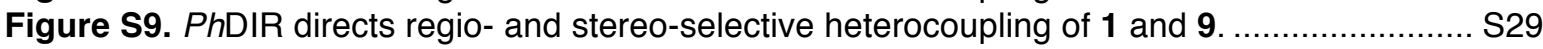
Figure S10. PhDIR mediates regio- and stereoselective homocoupling of 8,10 , and $11 \ldots \ldots \ldots \ldots$. S 30 
Figure S11. Two peaks separated by chiral chromatography are racemic S32

Figure S12. MS/MS fragmentation patterns support the structure assignment of 8-5' coupling products.

Figure S13. TVLac oxidation of 1 and 6 (1:9 molar ratio) at various $\mathrm{pH}$ does not alter the relative oxidation rates.

Figure S14. Time-course of TVLac oxidation of 1 and 6 at various ratios............................... S35

Figure S15. Optimization of PhDIR-mediated heterocoupling of $\mathbf{1}$ and $\mathbf{8}$ S36

Figure S16. Time-course of oxidative coupling reactions by TVLac in absence or presence of PhDIR

Figure S17. Standard curves of monolignols in TVLac oxidation assays .................................. S38

Figure S18. Representative full UV chromatograms of coupling reactions catalyzed by TVLac and mediated by PhDIR.

Table S1. Slopes and y-intercepts of linear equations from the standard curves S41

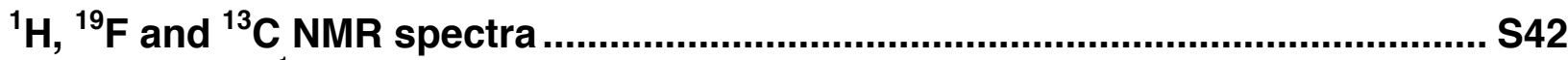

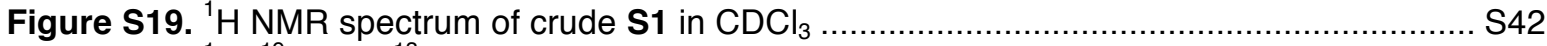

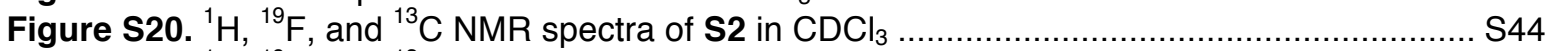

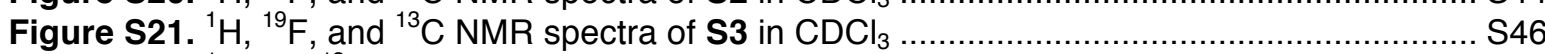

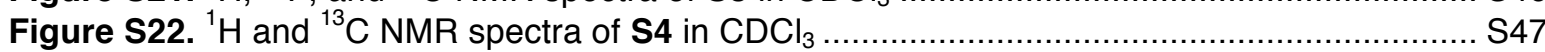

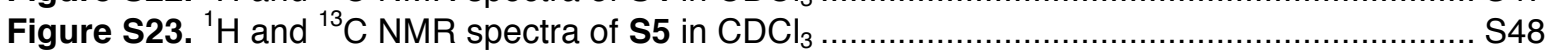

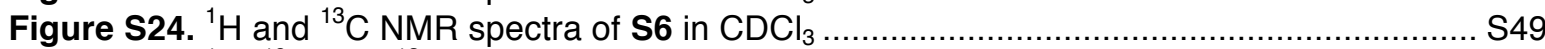

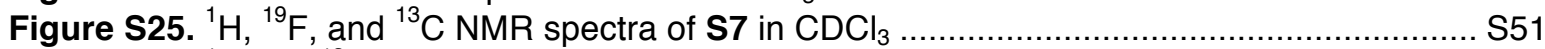

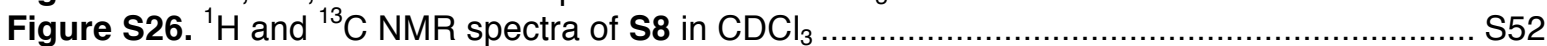

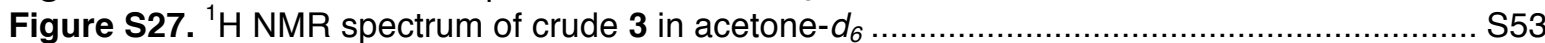

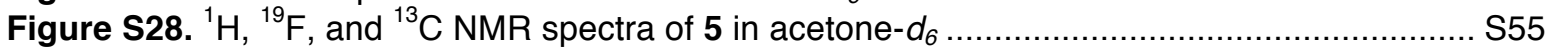

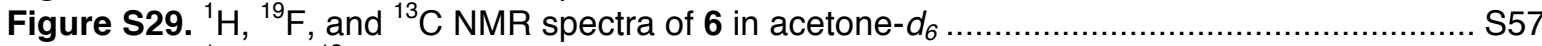

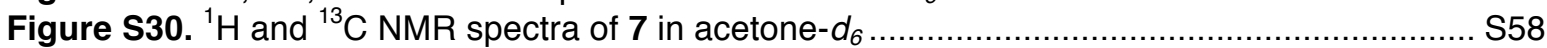

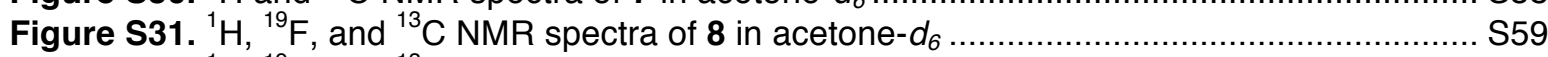

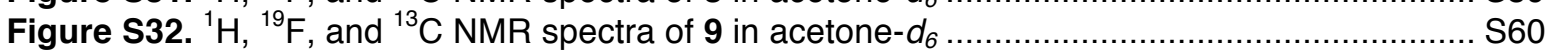

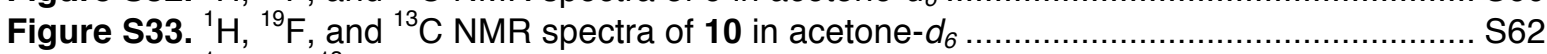

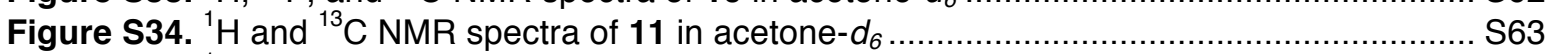

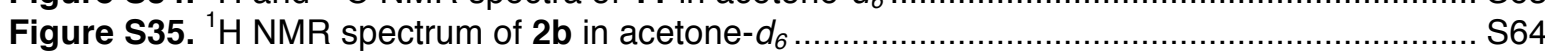

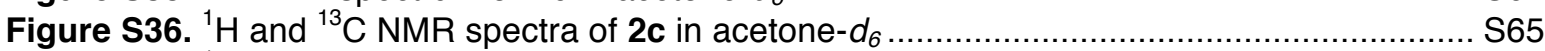

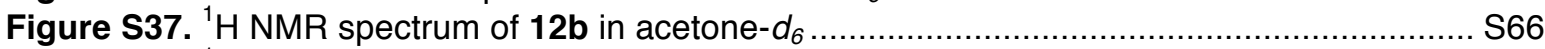

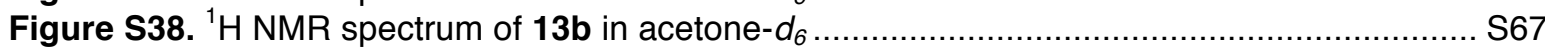

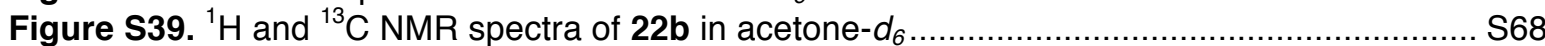

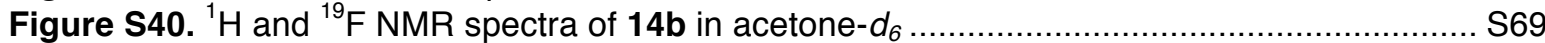

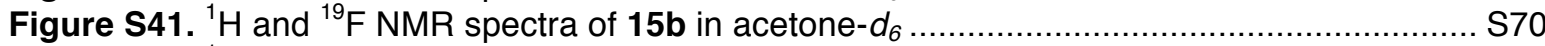

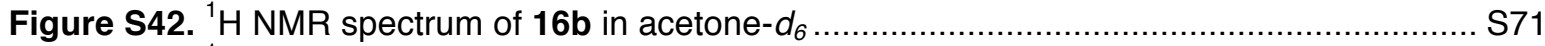

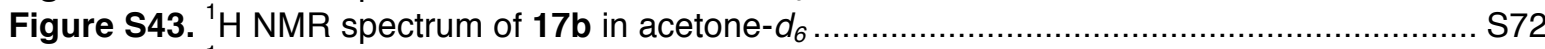

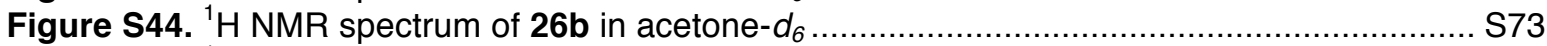

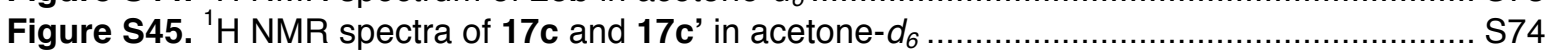

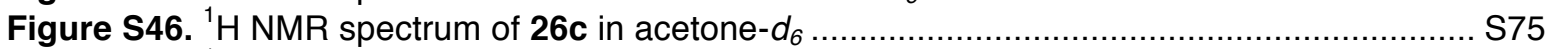

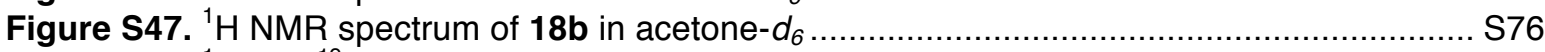

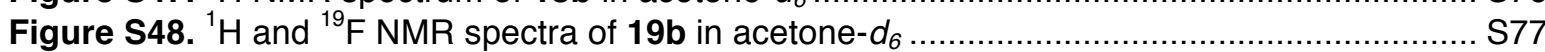

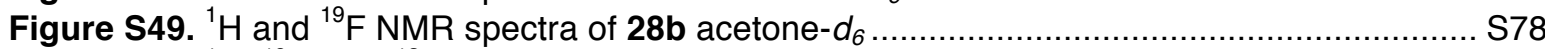

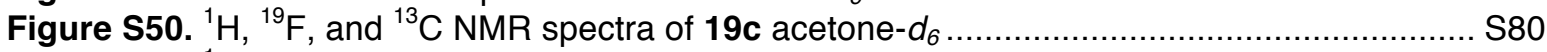

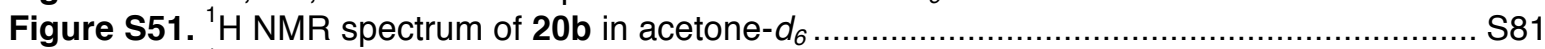

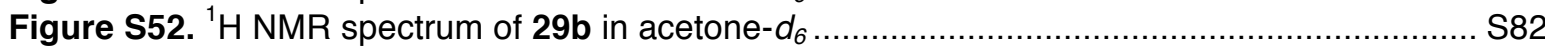

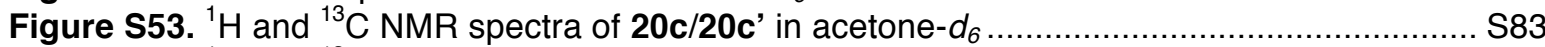

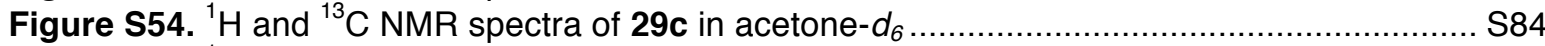

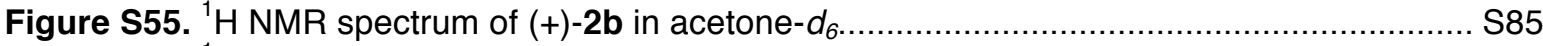

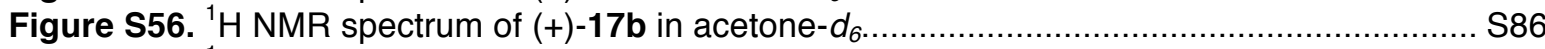

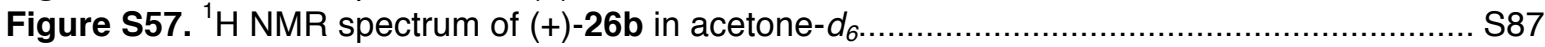




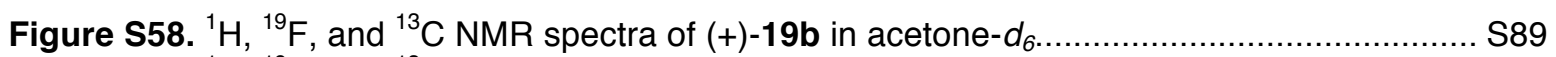

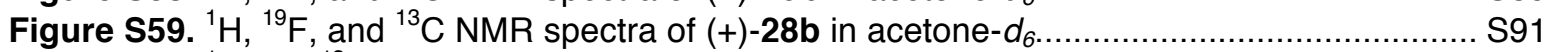

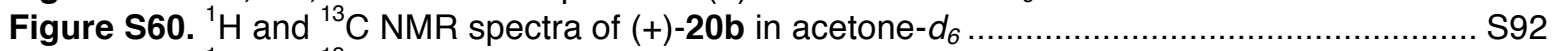

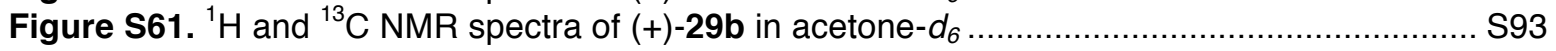

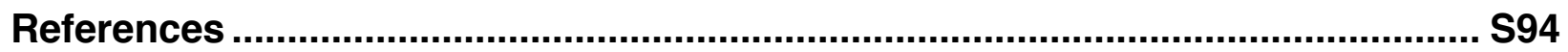




\section{Materials and Methods \\ General}

${ }^{1} \mathrm{H}$ NMR spectra were recorded at room temperature on a Varian $400(400 \mathrm{MHz})$, an Inova $500(500 \mathrm{MHz})$, or an Inova $600(600 \mathrm{MHz})$ spectrometer. Chemical shifts are reported in ppm downfield from tetramethylsilane using solvent resonance as internal standards $\left(\mathrm{CDCl}_{3}: \delta 7.26 \mathrm{ppm}\right.$ or acetone- $d_{6}: \delta 2.05$ ppm)

Proton-decoupled ${ }^{13} \mathrm{C}$ NMR spectra were recorded at room temperature on an Inova $500(126 \mathrm{MHz})$ or an Inova $600(151 \mathrm{MHz})$ spectrometer. Chemical shifts are reported in ppm downfield from tetramethylsilane using solvent resonance as internal standards (acetone- $d_{6}: \delta 29.84 \mathrm{ppm}$ ).

${ }^{19} \mathrm{~F}$ NMR spectra were recorded at room temperature on a Varian $400(376 \mathrm{MHz})$ or an Inova 500 (470 $\mathrm{MHz}$ ) spectrometer. Chemical shifts are reported in ppm, uncorrected, without an internal standard.

All synthetic, work-up and purification procedures were carried out with at least reagent-grade solvents. Standard column chromatography techniques with SilicaFlash ${ }^{\circledR}$ P60 silica gel $(40-63 \mu \mathrm{m})$ were used for purification unless otherwise noted.

\section{Materials}

Coniferyl alcohol (1, Ark Pharm), sinapyl alcohol (4, Santa Cruz Biotechnology), (+)-pinoresinol (2b, Sigma), 4-bromo-2-fluorophenol (Sigma-Aldrich), methyl acrylate (Aldrich), DIBAL-H (Aldrich), tri-o-tolyl-phosphine (Sigma-Aldrich), 4-bromo-2-(trifluoromethoxy)phenol (Alfa Aesar), 3-bromo-4-hydroxybenzaldehyde (Sigma-Aldrich), 4-hydroxy-3,5-dimethylbenzaldehyde (Alfa Aesar), 4-hydroxy-3-methylbenzaldehyde (Sigma-Aldrich), 3-fluoro-4-hydroxy-5-methoxybenzaldehyde (Matrix Scientific), 3-ethoxy-4hydroxybenzaldehyde (Acros), palladium diacetate (Sigma-Aldrich), (carbethoxymethylene)triphenylphosphorane (Sigma-Aldrich), p-coumaric acid (Alfa Aesar), and acyl chloride (Acros) were used as received without further purification. Monomers $\mathbf{3}$ and 5-11 were synthesized in house (see Synthetic Procedures below). Laccase from Trametes versicolor (TVac, Sigma; lot: 1438410V; activity: $660 \mathrm{mU} / \mathrm{mg}$ ), horseradish peroxide (HRP, GoldBio), hydrogen peroxide $\left(\mathrm{H}_{2} \mathrm{O}_{2}\right.$, Fisher) and ammonium persulfate (APS, TCl America) were used as received. $\mathrm{CDCl}_{3}$ and acetone- $d_{6}$ were purchased from Cambridge Isotope Laboratories.

\section{LC-MS analysis}

End-point enzyme assays were analyzed by reversed-phase column chromatography on a coupled Agilent 6520 Accurate-Mass q-ToF ESI mass spectrometer, using a $5 \mu \mathrm{m}, 2 \times 100 \mathrm{~mm}$ Gemini NX-C18 column (Phenomenex) and a $3 \mu \mathrm{m}, 4.6 \times 100 \mathrm{~mm}$ Chiralpak IC-3 column (Daicel). Kinetic assays were analyzed by reversed-phase column chromatography on a coupled Agilent 6545 Accurate-Mass q-ToF ESI mass spectrometer, using a $1.8 \mu \mathrm{m}, 2.1$ x $50 \mathrm{~mm}$ ZORBAX Eclipse Plus C18 Rapid Resolution HD column (Agilent) for regioisomeric separation and without column separation (column connector) for TVLac oxidation.

For $\mathrm{C} 18$ column separation, water with $0.1 \%$ formic acid $(\mathrm{A})$ and acetonitrile with $0.1 \%$ formic acid $(\mathrm{B})$ were used as mobile phases at a flow rate of $0.4 \mathrm{ml} / \mathrm{min}$ with the following gradient: $0-1 \mathrm{~min}, 3 \% \mathrm{~B} ; 1-21 \mathrm{~min}$, 3$50 \% \mathrm{~B} ; 21-22 \mathrm{~min}, 50-97 \% \mathrm{~B} ; 22-27 \mathrm{~min}, 97 \% \mathrm{~B} ; 27-28 \mathrm{~min}, 97-3 \% \mathrm{~B}$; and $28-32 \mathrm{~min}, 3 \% \mathrm{~B}$. MS parameters were as the following: mass range, $50-1700 \mathrm{~m} / \mathrm{z}$; drying gas, $300{ }^{\circ} \mathrm{C}$ and $11 \mathrm{~L} / \mathrm{min}$; nebulizer, 35 psig; capillary, $3500 \mathrm{~V}$; fragmentor, $150 \mathrm{~V}$; skimmer, $65 \mathrm{~V}$; octopole 1RF Vpp, $750 \mathrm{~V}$; $699.3 \mathrm{~ms}$ per spectrum. The eluent in the first minute of each run was discarded to avoid salt contamination in the mass spectrometer.

For chiral column separation, same mobile phases were used at a flow rate of $1 \mathrm{ml} / \mathrm{min}$ and a column temperature of $40{ }^{\circ} \mathrm{C}$ with the following gradient: 0-1 min, 3\% B; 1-14 min, 3-70\% B; $14-15 \mathrm{~min}, 70-95 \% \mathrm{~B}$; 15-18 $\mathrm{min}, 95 \% \mathrm{~B} ; 18-19 \mathrm{~min}, 95-3 \% \mathrm{~B}$; and $19-23 \mathrm{~min}, 3 \% \mathrm{~B}$. MS parameters were as the following: mass range, $102-750 \mathrm{~m} / \mathrm{z}$; drying gas, $300^{\circ} \mathrm{C}$ and $11 \mathrm{~L} / \mathrm{min}$; nebulizer, $35 \mathrm{psig}$; capillary, $3500 \mathrm{~V}$; fragmentor, $150 \mathrm{~V}$; skimmer, $65 \mathrm{~V}$; octopole $1 \mathrm{RF} \mathrm{Vpp}, 750 \mathrm{~V} ; 699.3 \mathrm{~ms}$ per spectrum. The eluent in the first 1.5 minutes of each run were discarded to avoid salt contamination in the mass spectrometer. 
For tandem mass spectrometry (MS/MS) analysis, 0, 10, 20 and $40 \mathrm{~V}$ collision energies were used with an $\mathrm{m} / \mathrm{z}$ window of 1.3 centered on the $\mathrm{m} / \mathrm{z}$ analyzed. Unless otherwise noted, MS/MS spectra shown are at 10 $\mathrm{V}$ collision energy.

For kinetic assays, same mobile phases were used at a flow rate of $0.8 \mathrm{ml} / \mathrm{min}$ and a column temperature of $50{ }^{\circ} \mathrm{C}$ with the following gradient: $0-51 \mathrm{~s}, 30-97 \% \mathrm{~B} ; 51-54 \mathrm{~s}, 97-30 \% \mathrm{~B}$; and $54-60 \mathrm{~s}, 30 \%$. MS parameters were as the following: mass range, $100-700 \mathrm{~m} / \mathrm{z}$; drying gas, $300{ }^{\circ} \mathrm{C}$ and $11 \mathrm{~L} / \mathrm{min}$; nebulizer, $35 \mathrm{psig}$; sheath gas, $350^{\circ} \mathrm{C}$ and $11 \mathrm{~L} / \mathrm{min}$; capillary, $3500 \mathrm{~V}$; nozzle, $1000 \mathrm{~V}$; fragmentor, $150 \mathrm{~V}$; skimmer, $65 \mathrm{~V}$; octopole 1RF Vpp, $750 \mathrm{~V} ; 699.3 \mathrm{~ms}$ per spectrum. The eluent in the first 6 seconds of each run was discarded to avoid salt contamination in the mass spectrometer.

\section{Preparative HPLC purification}

For preparative HPLC purification, an Agilent 1260 Infinity preparative-scale HPLC system with an Agilent 1100 diode array detector and a Clipeus C18 $10 \mu \mathrm{m} 250$ × 20 mm column (Higgins Analytical) was used. Unless otherwise noted, water with $0.1 \%$ formic acid $(A)$ and acetonitrile with $0.1 \%$ formic acid $(B)$ were used as mobile phase at a flow rate of $4.3 \mathrm{ml} / \mathrm{min}$ with the following method: $0-10 \mathrm{~min}, 20-30 \% \mathrm{~B} ; 10-60$ min, 30\% B isocratic; 60-65 min, 30-97\% B; and 65-80 min, 97\% B.

For mass-triggered fraction collection, an Agilent single quad MSD 6120 was used with a split ratio of 200:1 and an isopump (Agilent 1260 Infinity II) with a flow rate of $0.3 \mathrm{ml} / \mathrm{min}$ and dilution factor of $15: 1$. MS parameters were as the following: peak width, $0.1 \mathrm{~min}$; cycle time, $0.78 \mathrm{sec} / \mathrm{cycle}$; drying gas, $350^{\circ} \mathrm{C}$ and $12 \mathrm{~L} / \mathrm{min}$; nebulizer, $35 \mathrm{psig}$; capillary, $3000 \mathrm{~V}$. For multisignal acquisition, concurrent SIM/scan modes were used. Signal 1 (positive ion SIM mode, 50\% cycle time) on sample target masses with the following parameters: fragmentor, $70 \mathrm{~V}$; gain 1 . Signal 2 (positive ion scan mode, $50 \%$ cycle time) with the following parameters: mass range, 100-700 m/z; fragmentor, 70V; threshold, 150; step size 0.2; gain, 1; scan speed, $1300 \mathrm{u} / \mathrm{sec}$.

\section{Crude plant extraction for PhPLR}

$P$. hexandrum pinoresinol-lariciresinol reductase (PhPLR) was transiently expressed in $N$. benthamiana by agro-infiltration $\left(\mathrm{OD}_{600 \mathrm{~nm}}=0.3\right)$ for 5 days. Leaves were cut into halves, flash-frozen in liquid nitrogen, and homogenized in a ball-mill homogenizer (Retsch MM 400) at $25 \mathrm{~Hz}$ for $2 \mathrm{~min}$. Each half-leaf homogenate was resuspended in $500 \mu \mathrm{l}$ of ice-cold protein extraction buffer $(100 \mathrm{mM}$ Tris- $\mathrm{HCl}, \mathrm{pH} 7.5,10 \%$ glycerol, 1 $\mathrm{mM}$ PMSF, and $14 \mathrm{mM} \beta$-mercaptoethanol) and kept on ice for $30 \mathrm{~min}$ with occasional gentle inversion. The crude extract was transferred to a clean tube and centrifuged at $12,000 \mathrm{x} \mathrm{g}$ for $10 \mathrm{~min}$ at $4^{\circ} \mathrm{C}$ to clear cellular debris. The supernatant was ultrafiltrated (Amicon Ultra-0.5ml 10-kD) at 14,000 $\mathrm{g}$ g for $10 \mathrm{~min}$ at $4^{\circ} \mathrm{C}$. The retentate was aliquoted, flash-frozen in liquid nitrogen and stored at $-80^{\circ} \mathrm{C}$ until use at 5 -fold dilution.

\section{In-vitro LC-MS end-point analysis of combined PhDIR-PhPLR activity}

Crude PhDIR assay products prepared as described above were used directly. After incubation of the PhDIR reactions at $30^{\circ} \mathrm{C}$ for $40 \mathrm{~min}$, each reaction was diluted by 2.75 -fold to a final concentration of 40 $\mathrm{mM}$ Tris- $\mathrm{HCl}$ at $\mathrm{pH}$ 8.0, $0.9 \mathrm{mM} \mathrm{NADPH}$, and 5-fold diluted PLR crude extract concentrate, with water. Reactions were incubated at $30^{\circ} \mathrm{C}$ for $2 \mathrm{~h}$ and quenched with an equal volume of acetonitrile. The quenched reactions were centrifuged at $15,000 \mathrm{rpm}$ for $10 \mathrm{~min}$, and the supernatants were filtered for LC-MS analysis on both the positive and negative ion modes. 


\section{Synthetic procedures}

\section{monolignols}

p-coumaryl methyl ester, $\mathbf{S} \mathbf{1}$

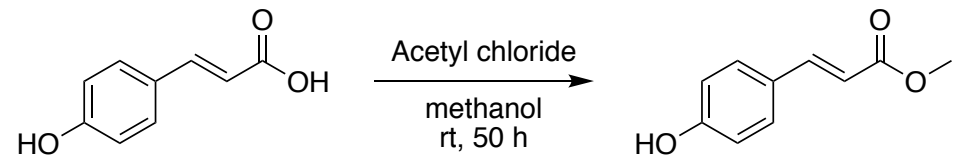

S1

Compound $\mathbf{S 1}$ was prepared by Fischer esterification, following the protocols by Quideau et al. ${ }^{1}$ To a solution of $p$-coumaric acid $(5.0 \mathrm{~g}, 30.5 \mathrm{mmol}, 1$ equiv) in $100 \mathrm{ml}$ methanol, acetyl chloride $(6.0 \mathrm{ml}, 84.6$ mmol, 2.8 equiv) was added. After stirring for $1 \mathrm{~h}$ at $\mathrm{rt}$, an additional portion of acetyl chloride $(5.0 \mathrm{ml}, 70.5$ $\mathrm{mmol}, 2.3$ equiv) was added. After $50 \mathrm{~h}$ of stirring at $\mathrm{rt}$, the reaction mixture was concentrated in vacuo and used without purification. TLC (EtOAc/hexanes, $2: 1 \mathrm{v} / \mathrm{v}): \mathrm{R}_{\mathrm{f}}=0.55 .{ }^{1} \mathrm{H} \mathrm{NMR}\left(500 \mathrm{MHz}, \mathrm{CDCl}_{3}\right) \delta 7.64(\mathrm{~d}, J$ $=16.0 \mathrm{~Hz}, 1 \mathrm{H}), 7.43(\mathrm{~d}, J=8.8 \mathrm{~Hz}, 2 \mathrm{H}), 6.85(\mathrm{~d}, J=8.8 \mathrm{~Hz}, 2 \mathrm{H}), 6.31(\mathrm{~d}, J=16.0 \mathrm{~Hz}, 1 \mathrm{H}), 3.80(\mathrm{~s}, 3 \mathrm{H})$. All spectroscopic data were consistent with reported literature values. ${ }^{2}$

methyl (E)-3-(4-hydroxy-3-(trifluoromethoxy)phenyl)acrylate, $\mathbf{S 2}$

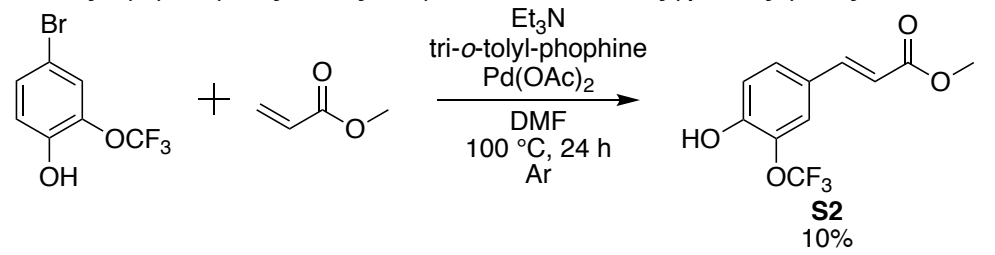

Compound $\mathbf{S 2}$ was prepared by Heck reaction, following the protocols by Ramamoorthy et al. ${ }^{3}$ with slight modifications. Briefly, to a solution of bromo-trifluoromethoxy-phenol (500 mg, $1.95 \mathrm{mmol}, 1$ equiv) and methyl acrylate ( $350 \mu \mathrm{l}, 3.89 \mathrm{mmol}, 2$ equiv) in $7.9 \mathrm{ml}$ dry DMF, triethylamine (542 $\mu \mathrm{l}$, 2 equiv), tri-o-tolylphosphine ( $30 \mathrm{mg}, 0.05$ equiv), and palladium diacetate ( $44 \mathrm{mg}, 0.1$ equiv) were added. The mixture was degassed for 30 min by bubbling argon through while stirring and then placed in an oil bath pre-heated to $100{ }^{\circ} \mathrm{C}$ and stirred overnight. At this time, palladium catalysts were removed from the crude mixture by vacuum filtration, and the filtrate was diluted with toluene and concentrated in vacuo. This step was repeated three times to remove DMF via low-boiling azeotrope. The resulting residue was diluted with ethyl acetate and successively washed with water and brine solution. The first water wash was then backextracted three times with ethyl acetate. The organic layer was dried over anhydrous sodium sulfate, gravity filtered, and concentrated in vacuo. The crude residue was purified by silica gel flash column chromatography eluting with a gradient from 20 to $40 \%$ EtOAc/hexanes to yield $\mathbf{S 2}$ as a light-yellow powder $(51.9 \mathrm{mg}, 10 \%$ yield) TLC (EtOAc/hexanes, $2: 3 \mathrm{v} / \mathrm{v}): \mathrm{R}_{\mathrm{f}}=0.45 .{ }^{1} \mathrm{H}$ NMR $\left(400 \mathrm{MHz}, \mathrm{CDCl}_{3}\right) \delta 7.59(\mathrm{~d}, J=$ $16.0 \mathrm{~Hz}, 1 \mathrm{H}), 7.43-7.32(\mathrm{~m}, 2 \mathrm{H}), 7.05(\mathrm{~d}, J=8.8 \mathrm{~Hz}, 1 \mathrm{H}), 6.32(\mathrm{~d}, J=16.0 \mathrm{~Hz}, 1 \mathrm{H}), 6.01(\mathrm{~s}, 1 \mathrm{H}), 3.81(\mathrm{~s}$, 3H). ${ }^{19} \mathrm{~F} \mathrm{NMR}\left(470 \mathrm{MHz}, \mathrm{CDCl}_{3}\right) \delta-57.95$ (s, 3F). ${ }^{13} \mathrm{C} \mathrm{NMR}\left(126 \mathrm{MHz}, \mathrm{CDCl}_{3}\right) \delta 167.4,149.6,143.3,136.7$, 128.2, 127.9, 121.0, 120.7 (q, $J=259.6 \mathrm{~Hz}), 117.9,117.2,51.9$.

methyl (E)-3-(3-fluoro-4-hydroxyphenyl)acrylate, S3

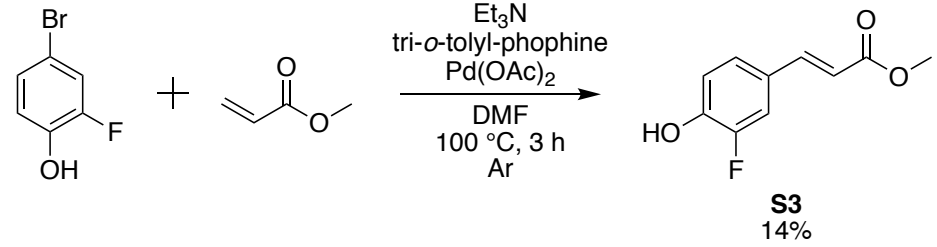

Compound $\mathbf{S 3}$ was prepared following similar protocols as $\mathbf{S 2}$, yielding S3 as a light-yellow powder (210.5 $\mathrm{mg}, 14 \%$ yield) TLC (EtOAc/hexanes, 2:3 v/v): $\mathrm{R}_{\mathrm{f}}=0.45 .{ }^{1} \mathrm{H}$ NMR $\left(400 \mathrm{MHz}, \mathrm{CDCl}_{3}\right) \delta 7.58(\mathrm{~d}, \mathrm{~J}=16.0 \mathrm{~Hz}$, $1 \mathrm{H}), 7.26(\mathrm{dd}, J=11.4,2.0 \mathrm{~Hz}, 1 \mathrm{H}), 7.23-7.17(\mathrm{~m}, 1 \mathrm{H}), 7.00(\mathrm{app} \mathrm{t}, J=8.7 \mathrm{~Hz}, 1 \mathrm{H}), 6.29(\mathrm{~d}, J=16.0 \mathrm{~Hz}$, $1 \mathrm{H}), 6.17(\mathrm{~s}, 1 \mathrm{H}), 3.80(\mathrm{~s}, 3 \mathrm{H}) .{ }^{19} \mathrm{~F} \mathrm{NMR}\left(470 \mathrm{MHz}, \mathrm{CDCl}_{3}\right) \delta-139.76(\mathrm{dd}, J=11.4,8.7 \mathrm{~Hz}) .{ }^{13} \mathrm{C} \mathrm{NMR}(126$ 
$\left.\mathrm{MHz}, \mathrm{CDCl}_{3}\right) \delta 167.6,151.2(\mathrm{~d}, J=238.9 \mathrm{~Hz}), 145.7(\mathrm{~d}, J=14.6 \mathrm{~Hz}), 143.7(\mathrm{~d}, J=2.3 \mathrm{~Hz}), 127.9(\mathrm{~d}, J=$ $6.4 \mathrm{~Hz}$ ), $125.6(\mathrm{~d}, J=3.0 \mathrm{~Hz}), 117.7(\mathrm{~d}, J=1.9 \mathrm{~Hz}), 116.8,114.7(\mathrm{~d}, J=18.6 \mathrm{~Hz}), 51.8$. All spectroscopic data were consistent with reported literature values. ${ }^{3}$

ethyl (E)-3-(3-bromo-4-hydroxyphenyl)acrylate, S4

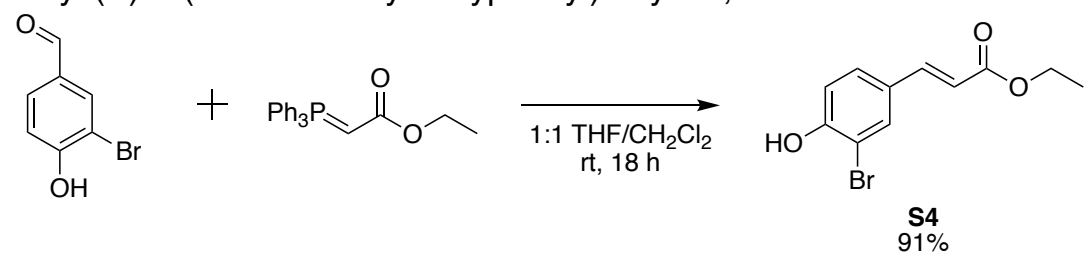

Compound S4 was prepared by an E-selective olefination protocol adapted from the works of RomanovMichailidis et al. ${ }^{4}$ Briefly, to a solution of ethyl (triphenyl-phosphoranylidene)acetate $(1.04 \mathrm{~g}, 2.99 \mathrm{mmol}, 1.2$ equiv) in $4.2 \mathrm{ml}$ dry THF and $4.2 \mathrm{ml}$ dry $\mathrm{CH}_{2} \mathrm{Cl}_{2}$, 3-bromo-4-hydroxybenzaldehyde (500 mg, $2.49 \mathrm{mmol}, 1$ equiv) was added. Upon dissolution of the benzaldehyde, the reaction turned dark yellow. The reaction was allowed to stir at rt overnight. At this time, the solvent was removed in vacuo, and the residue was purified by silica gel flash column chromatography eluting with 20\% EtOAc/hexanes to yield $\mathbf{S} 4$ as a white powder (616 mg, 91.3\% yield). TLC (EtOAc/hexanes, $1: 3 \mathrm{v} / \mathrm{v}): \mathrm{Rf}=0.32 .{ }^{1} \mathrm{H}$ NMR $\left(500 \mathrm{MHz}, \mathrm{CDCl}_{3}\right) \delta 7.65(\mathrm{~d}, J=$ $2.1 \mathrm{~Hz}, 1 \mathrm{H}), 7.55(\mathrm{~d}, J=16.0 \mathrm{~Hz}, 1 \mathrm{H}), 7.40(\mathrm{dd}, J=8.4,2.1 \mathrm{~Hz}, 1 \mathrm{H}), 7.02(\mathrm{~d}, J=8.4 \mathrm{~Hz}, 1 \mathrm{H}), 6.30(\mathrm{~d}, J=$ $16.0 \mathrm{~Hz}, 1 \mathrm{H}), 5.84(\mathrm{~s}, 1 \mathrm{H}), 4.25(\mathrm{q}, J=7.1 \mathrm{~Hz}, 2 \mathrm{H}), 1.33(\mathrm{t}, J=7.1 \mathrm{~Hz}, 3 \mathrm{H}) .{ }^{13} \mathrm{C} \mathrm{NMR}\left(126 \mathrm{MHz}, \mathrm{CDCl}_{3}\right) \delta$ $167.1,154.0,142.8,131.9,129.2,128.9,117.4,116.6,110.9,60.7,14.5$.

ethyl (E)-3-(4-hydroxy-3-methylphenyl)acrylate, $\mathbf{5 5}$

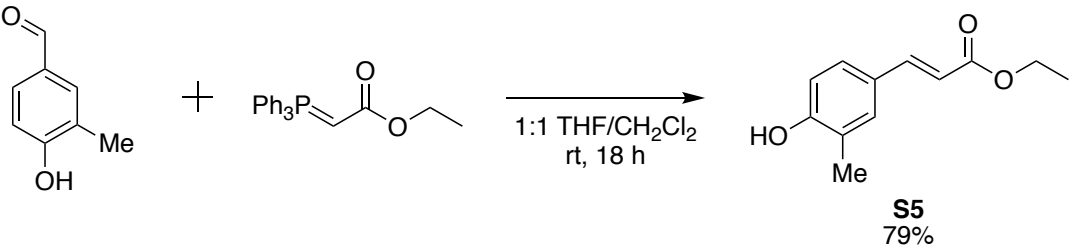

Compound S5 was prepared following similar protocols as $\mathbf{S 4}$ and purified by silica gel flash column chromatography eluting with a gradient from 5\% to 10\% EtOAc/hexanes to yield S5 as a white powder (599.2 mg, 79.2\% yield) TLC (EtOAc/hexanes, 1:4 v/v): $\mathrm{R}_{\mathrm{f}}=0.25 .{ }^{1} \mathrm{H}$ NMR $\left(600 \mathrm{MHz}, \mathrm{CDCl}_{3}\right) \delta 7.56(\mathrm{~d}, J$ $=16.0 \mathrm{~Hz}, 1 \mathrm{H}), 7.20(\mathrm{~d}, J=8.4 \mathrm{~Hz}, 1 \mathrm{H}), 6.75(\mathrm{~d}, J=8.4 \mathrm{~Hz}, 1 \mathrm{H}), 6.24(\mathrm{~d}, J=16.0 \mathrm{~Hz}, 1 \mathrm{H}), 5.94(\mathrm{~s}, 1 \mathrm{H})$, $4.21(\mathrm{q}, J=7.1 \mathrm{~Hz}, 2 \mathrm{H}), 2.21(\mathrm{~s}, 3 \mathrm{H}), 1.29(\mathrm{t}, J=7.1 \mathrm{~Hz}, 3 \mathrm{H}) .{ }^{13} \mathrm{C} \mathrm{NMR}\left(151 \mathrm{MHz}, \mathrm{CDCl}_{3}\right) \delta 168.1,156.5$, $145.1,131.2,127.7,127.1,124.8,115.5,115.2,60.7,15.9,14.5$.

ethyl (E)-3-(4-hydroxy-3,5-dimethylphenyl)acrylate, $\mathbf{S 6}$

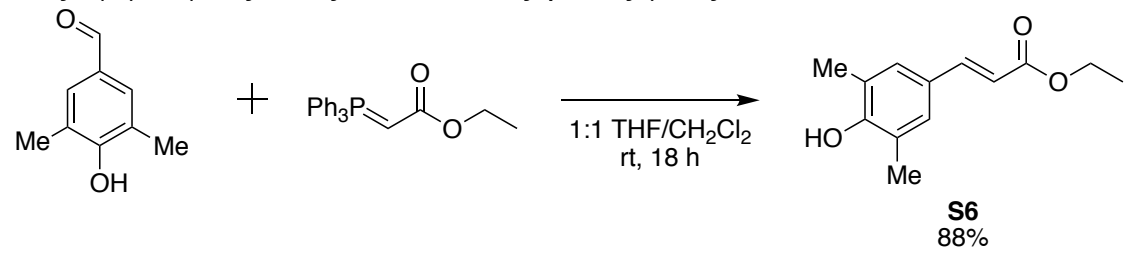

Compound $\mathbf{S 6}$ was prepared following similar protocols as $\mathbf{S 4}$ and purified by silica gel flash column chromatography eluting with $10 \% \mathrm{EtOAc} /$ hexanes to yield $\mathbf{S 6}$ as a white powder $(644.1 \mathrm{mg}, 87.9 \%$ yield) TLC (EtOAc/hexanes, 1:4 v/v): $R_{f}=0.29 .{ }^{1} \mathrm{H}$ NMR $\left(500 \mathrm{MHz}, \mathrm{CDCl}_{3}\right) \delta 7.58(\mathrm{~d}, J=16.0 \mathrm{~Hz}, 1 \mathrm{H}), 7.17(\mathrm{~s}$, $2 \mathrm{H}), 6.28(\mathrm{~d}, J=16.0 \mathrm{~Hz}, 1 \mathrm{H}), 5.23(\mathrm{~s}, 1 \mathrm{H}), 4.25(\mathrm{q}, J=7.2 \mathrm{~Hz}, 2 \mathrm{H}), 2.25(\mathrm{~s}, 6 \mathrm{H}), 1.33(\mathrm{t}, J=7.2 \mathrm{~Hz}, 3 \mathrm{H})$. ${ }^{13} \mathrm{C}$ NMR $\left(126 \mathrm{MHz}, \mathrm{CDCl}_{3}\right) \delta 167.8,154.6,145.0,131.3,129.0,123.7,115.3,60.4,16.0,14.5$. 
ethyl (E)-3-(3-fluoro-4-hydroxy-5-methoxyphenyl)acrylate, S7

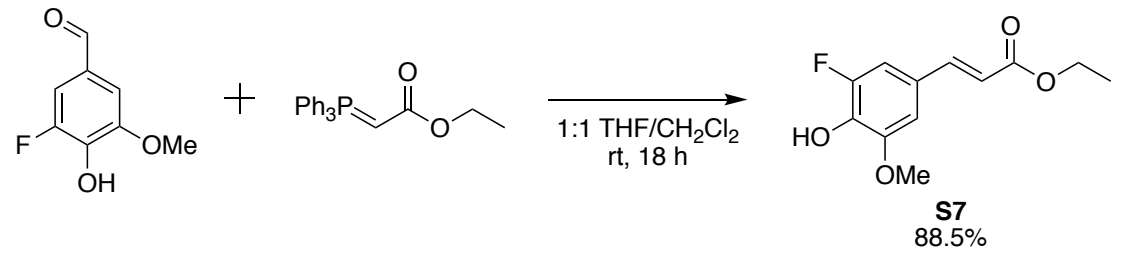

Compound $\mathbf{S 7}$ was prepared following similar protocols as S4 and purified by silica gel flash column chromatography eluting with a gradient from $10 \%$ to $20 \%$ EtOAc/hexanes to yield S7 as a white powder (624.9 mg, 88.5\% yield). TLC (EtOAc/hexanes, $2: 3 \mathrm{v} / \mathrm{v}): \mathrm{R}_{\mathrm{f}}=0.38 .{ }^{1} \mathrm{H}$ NMR $\left(400 \mathrm{MHz}, \mathrm{CDCl}_{3}\right) \delta 7.54(\mathrm{~d}, J$ $=15.9 \mathrm{~Hz}, 1 \mathrm{H}), 6.94(\mathrm{dd}, J=10.9,1.8 \mathrm{~Hz}, 1 \mathrm{H}), 6.83(\mathrm{app} \mathrm{t}, J=1.8 \mathrm{~Hz}, 1 \mathrm{H}), 6.28(\mathrm{~d}, J=15.9 \mathrm{~Hz}, 1 \mathrm{H}), 5.79$ (s, $1 \mathrm{H}), 4.25(\mathrm{q}, J=7.1 \mathrm{~Hz}, 2 \mathrm{H}), 3.93(\mathrm{~s}, 3 \mathrm{H}), 1.33(\mathrm{t}, J=7.1 \mathrm{~Hz}, 3 \mathrm{H}) .{ }^{19} \mathrm{~F} \mathrm{NMR}\left(470 \mathrm{MHz}, \mathrm{CDCl}_{3}\right) \delta-136.59$ (d, $J=10.9 \mathrm{~Hz}) \cdot{ }^{13} \mathrm{C}$ NMR $\left(126 \mathrm{MHz}, \mathrm{CDCl}_{3}\right) \delta 167.0,150.6(\mathrm{~d}, J=241.8 \mathrm{~Hz}), 148.4(\mathrm{~d}, J=6.0 \mathrm{~Hz}), 143.7$ (d, $J=2.4 \mathrm{~Hz}$ ), 135.9 (d, $J=13.9 \mathrm{~Hz}), 126.2(\mathrm{~d}, J=8.3 \mathrm{~Hz}), 117.3,109.5$ (d, $J=19.1 \mathrm{~Hz}), 106.1(\mathrm{~d}, J=1.9$ $\mathrm{Hz}), 60.6,56.6,14.4$.

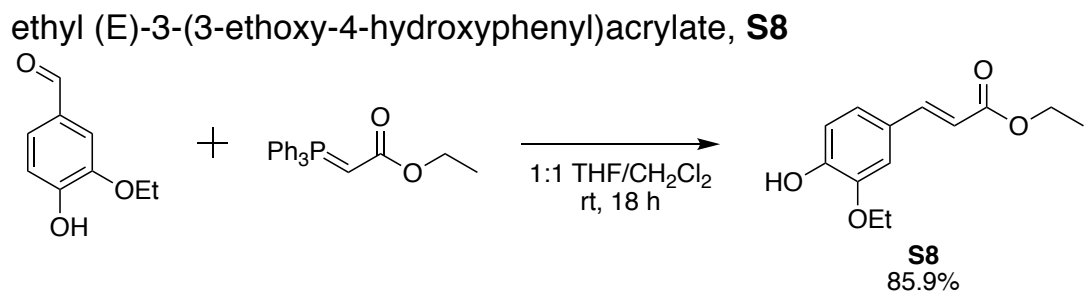

Compound S8 was prepared following similar protocols as S4 and purified by silica gel flash column chromatography eluting with $10 \%$ EtOAc/hexanes to yield $\mathbf{S 8}$ as a white powder (609 $\mathrm{mg}, 85.9 \%$ yield) TLC (EtOAc/hexanes, 2:3 v/v): $R_{f}=0.43 .{ }^{1} \mathrm{H}$ NMR $\left(500 \mathrm{MHz}, \mathrm{CDCl}_{3}\right) \delta 7.59(\mathrm{~d}, J=15.9 \mathrm{~Hz}, 1 \mathrm{H}), 7.04(\mathrm{dd}, J=$ $8.2,1.9 \mathrm{~Hz}, 1 \mathrm{H}), 7.00(\mathrm{~d}, J=1.9 \mathrm{~Hz}, 1 \mathrm{H}), 6.91(\mathrm{~d}, J=8.2 \mathrm{~Hz}, 1 \mathrm{H}), 6.26(\mathrm{~d}, J=15.9 \mathrm{~Hz}, 1 \mathrm{H}), 6.06(\mathrm{~s}, 1 \mathrm{H})$, $4.24(\mathrm{q}, J=7.1 \mathrm{~Hz}, 2 \mathrm{H}), 4.12(\mathrm{q}, J=7.0 \mathrm{~Hz}, 2 \mathrm{H}), 1.45(\mathrm{t}, J=7.0 \mathrm{~Hz}, 3 \mathrm{H}), 1.32(\mathrm{t}, J=7.1 \mathrm{~Hz}, 3 \mathrm{H}) .{ }^{13} \mathrm{C}$ NMR $\left(126 \mathrm{MHz}, \mathrm{CDCl}_{3}\right) \delta 167.4,148.1,146.1,144.8,127.0,122.9,115.5,114.7,110.3,64.6,60.4,14.8,14.4$.

p-coumaryl alcohol, 3

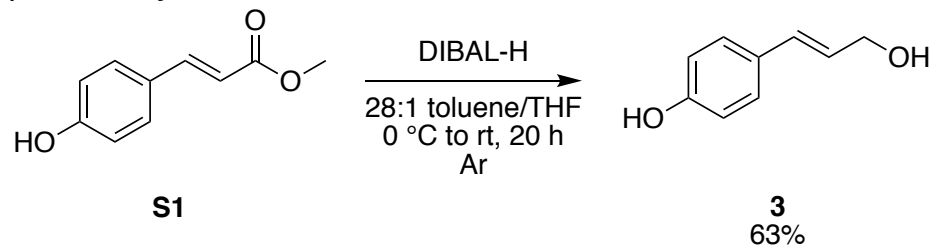

Compound $\mathbf{3}$ was prepared by DIBAL-H reduction of $\mathbf{S} 1$ to the corresponding alcohol, following similar protocols by Quideau et al. ${ }^{1}$ A pre-dried round bottom flask was charged with crude $\mathbf{S} 1(1.0 \mathrm{~g}, 5.6 \mathrm{mmol}$, 1.0 equiv) and put under argon. Dry toluene $(56 \mathrm{ml})$ and dry THF $(2 \mathrm{ml})$ were added via syringe, and the solution was cooled to $0^{\circ} \mathrm{C}$ in an ice bath. DIBAL-H (neat, $4.0 \mathrm{ml}, 22.5 \mathrm{mmol}, 4.0$ equiv) was added dropwise via syringe at $0{ }^{\circ} \mathrm{C}$ over $3 \mathrm{~min}$. Upon complete addition, the ice bath was removed, and the reaction stirred at $\mathrm{rt}$ for $20 \mathrm{~h}$. At this time, the reaction was quenched with 10\% Rochelle's salt solution and extracted with ethyl acetate. The organic layer was dried over anhydrous sodium sulfate, gravity filtered, and concentrated in vacuo. The crude residue was purified by silica gel flash column chromatography eluting with $40 \%$ EtOAc/hexanes to yield 3 as a light-yellow powder $(530 \mathrm{mg}, 63 \%$ yield). TLC (EtOAc/hexanes, $2: 1 \mathrm{v} / \mathrm{v}): \mathrm{R}_{\mathrm{f}}=0.27 .{ }^{1} \mathrm{H} \mathrm{NMR}\left(400 \mathrm{MHz}\right.$, Acetone- $\left.d_{6}\right) \delta 8.35(\mathrm{~s}, 1 \mathrm{H}), 7.27(\mathrm{~d}, J=8.6 \mathrm{~Hz}, 2 \mathrm{H}), 6.79(\mathrm{~d}, J=8.6$ $\mathrm{Hz}, 2 \mathrm{H}), 6.50(\mathrm{dt}, J=15.9,1.6 \mathrm{~Hz}, 1 \mathrm{H}), 6.20(\mathrm{dtd}, J=15.9,5.6,0.8 \mathrm{~Hz}, 1 \mathrm{H}), 4.19$ (td, $J=5.6,1.6 \mathrm{~Hz}, 2 \mathrm{H})$, $3.77-3.69(\mathrm{~m}, 1 \mathrm{H})$. HRMS $(\mathrm{m} / \mathrm{z}):\left[\mathrm{M}-\mathrm{H}_{2} \mathrm{O}+\mathrm{H}\right]^{+}$calcd. for $\mathrm{C}_{9} \mathrm{H}_{9} \mathrm{O}^{+}, 133.0648$; found, 133.0647. All spectroscopic data were consistent with reported literature values. ${ }^{1}$ 
3-trifluoromethoxy p-coumaryl alcohol, 5<smiles>COC(=O)/C=C/c1ccc(O)c(OC(F)(F)F)c1</smiles>

Compound $\mathbf{5}$ was prepared by DIBAL-H reduction of $\mathbf{S 2}$ to the corresponding alcohol. A solution of $\mathbf{S 2}(44.4$ $\mathrm{mg}, 0.169 \mathrm{mmol}, 1$ equiv) in $1.1 \mathrm{ml}$ dry toluene and $50 \mu \mathrm{l}$ dry THF was stirred at $-78^{\circ} \mathrm{C}$ while being degassed with argon for $15 \mathrm{~min}$. A solution of DIBAL-H (1.5 M in toluene, $564 \mu \mathrm{l}, 0.847 \mathrm{mmol}, 5$ equiv) was added dropwise. Upon completion of DIBAL-H addition, the reaction was brought to rt. After $5 \mathrm{~h}$, the reaction was quenched with ethyl acetate under argon and diluted with $100 \mathrm{ml}$ of 1:1 ethyl acetate:sat'd Rochelle's salt solution. The emulsion was vigorously stirred overnight. At this time, the separated organic layer was dried over anhydrous sodium sulfate, gravity filtered, and concentrated in vacuo. The crude residue was purified by silica gel flash column chromatography eluting with $40 \%$ EtOAc/hexanes to yield $\mathbf{5}$ as a light-yellow powder $\left(29.9 \mathrm{mg}, 75.5 \%\right.$ yield). ${ }^{1} \mathrm{H}$ NMR $\left(500 \mathrm{MHz}\right.$, Acetone- $\left.d_{6}\right) \delta 9.03(\mathrm{~s}, 1 \mathrm{H}), 7.33(\mathrm{t}, J=2.1 \mathrm{~Hz}, 1 \mathrm{H})$, $7.29(\mathrm{dd}, J=8.4,2.1 \mathrm{~Hz}, 1 \mathrm{H}), 7.02(\mathrm{~d}, J=8.4 \mathrm{~Hz}, 1 \mathrm{H}), 6.55(\mathrm{dt}, J=15.9,1.7 \mathrm{~Hz}, 1 \mathrm{H}), 6.30(\mathrm{dt}, J=15.9$, $5.3 \mathrm{~Hz}, 1 \mathrm{H}), 4.21(\mathrm{dd}, J=5.3,1.7 \mathrm{~Hz}, 2 \mathrm{H}), 3.90(\mathrm{~s}, 1 \mathrm{H}) .{ }^{19} \mathrm{~F} \mathrm{NMR}\left(470 \mathrm{MHz}\right.$, Acetone- $\left.d_{6}\right) \delta-59.22 .{ }^{13} \mathrm{C}$ NMR $\left(126 \mathrm{MHz}\right.$, Acetone- $\left.d_{6}\right) \delta 149.8,137.6,130.9,130.1,128.5,127.0,122.8(q, J=255.5 \mathrm{~Hz}), 121.3$, 118.7, 63.1. HRMS (m/z): $\left[\mathrm{M}-\mathrm{H}_{2} \mathrm{O}+\mathrm{H}\right]^{+}$calcd. for $\mathrm{C}_{10} \mathrm{H}_{8} \mathrm{~F}_{3} \mathrm{O}_{2}{ }^{+}, 217.0471$; found, 217.0464 .

3-fluoro p-coumaryl alcohol, 6<smiles>COC(=O)/C=C/c1ccc(O)c(F)c1</smiles>

Compound $\mathbf{6}$ was prepared by DIBAL-H reduction of $\mathbf{S} 3$ into alcohol following similar protocols as $\mathbf{5}$, using neat DIBAL-H, to give 6 as a light-yellow powder $\left(159.8 \mathrm{mg}, 96 \%\right.$ yield). ${ }^{1} \mathrm{H}$ NMR $\left(500 \mathrm{MHz}\right.$, Acetone- $\left.d_{6}\right) \delta$ $8.69(\mathrm{~s}, 1 \mathrm{H}), 7.19(\mathrm{dd}, J=12.5,2.1 \mathrm{~Hz}, 1 \mathrm{H}), 7.06(\mathrm{dd}, J=8.4,2.1 \mathrm{~Hz}, 1 \mathrm{H}), 6.93(\mathrm{app} \mathrm{t}, J=8.4 \mathrm{~Hz}, 1 \mathrm{H})$, $6.50(\mathrm{dt}, J=15.9,1.7 \mathrm{~Hz}, 1 \mathrm{H}), 6.26(\mathrm{dt}, J=15.9,5.4 \mathrm{~Hz}, 1 \mathrm{H}), 4.21(\mathrm{dd}, J=5.4,1.7 \mathrm{~Hz}, 1 \mathrm{H}), 3.92(\mathrm{~s}, 1 \mathrm{H})$. ${ }^{19} \mathrm{~F}$ NMR $\left(470 \mathrm{MHz}\right.$, Acetone- $\left.d_{6}\right) \delta-139.05(\mathrm{dd}, J=12.5,8.4 \mathrm{~Hz}) .{ }^{13} \mathrm{C}$ NMR $(126 \mathrm{MHz}$, Acetone) $\delta 152.4(\mathrm{~d}$, $J=239.7 \mathrm{~Hz}$ ), $145.0(\mathrm{~d}, J=13.4 \mathrm{~Hz}), 130.9(\mathrm{~d}, J=6.2 \mathrm{~Hz}), 129.6,128.9(\mathrm{~d}, J=2.2 \mathrm{~Hz}), 123.7(\mathrm{~d}, J=3.1$ $\mathrm{Hz}), 118.6(\mathrm{~d}, J=3.1 \mathrm{~Hz}), 114.1(\mathrm{~d}, J=18.6 \mathrm{~Hz}), 63.2$. $\mathrm{HRMS}(\mathrm{m} / \mathrm{z}):\left[\mathrm{M}-\mathrm{H}_{2} \mathrm{O}+\mathrm{H}\right]^{+}$calcd. for $\mathrm{C}_{9} \mathrm{H}_{8} \mathrm{FO}^{+}$, 151.0554; found, 151.0561 .

3-bromo p-coumaryl alcohol, 7

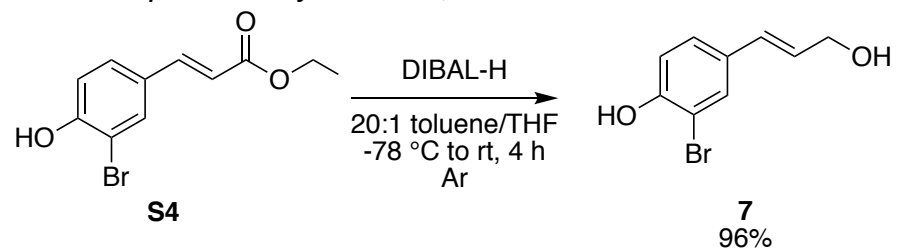

Compound $\mathbf{7}$ was prepared by DIBAL-H reduction of $\mathbf{S} \mathbf{4}$ into alcohol following similar protocols as $\mathbf{5}$ and purified by silica gel flash column chromatography eluting with $30 \%$ EtOAc/hexanes to yield 7 as a white powder (161.9 mg, 95.8\% yield). TLC (EtOAc/hexanes, 2:3 v/v): $R_{f}=0.22 .{ }^{1} \mathrm{H}$ NMR (500 MHz, Acetone- $\left.d_{6}\right)$ $\delta 8.90(\mathrm{~s}, 1 \mathrm{H}), 7.56(\mathrm{~d}, J=2.2 \mathrm{~Hz}, 1 \mathrm{H}), 7.28(\mathrm{dd}, J=8.4,2.2 \mathrm{~Hz}, 1 \mathrm{H}), 6.96(\mathrm{~d}, J=8.4 \mathrm{~Hz}, 1 \mathrm{H}), 6.50(\mathrm{dt}, J$ $=15.9,1.8 \mathrm{~Hz}, 1 \mathrm{H}), 6.27(\mathrm{dt}, J=15.9,5.4 \mathrm{~Hz}, 1 \mathrm{H}), 4.21(\mathrm{dd}, J=5.4,1.8 \mathrm{~Hz}, 2 \mathrm{H}), 3.90(\mathrm{~s}, 1 \mathrm{H}) .{ }^{13} \mathrm{C} \mathrm{NMR}$ $\left(126 \mathrm{MHz}\right.$, Acetone- $\left.d_{6}\right) \delta 154.1,131.8,131.5,129.7,128.4,127.4,117.3,110.6,63.1 . \mathrm{HRMS}$ (m/z): [M$\left.\mathrm{H}_{2} \mathrm{O}+\mathrm{H}\right]^{+}$calcd. for $\mathrm{C}_{9} \mathrm{H}_{8} \mathrm{BrO}^{+}, 210.9753\left({ }^{79} \mathrm{Br}\right)$ and $212.9733\left({ }^{81} \mathrm{Br}\right)$; found, 210.9738 and 212.9718 . 
3-methyl p-coumaryl alcohol, 8

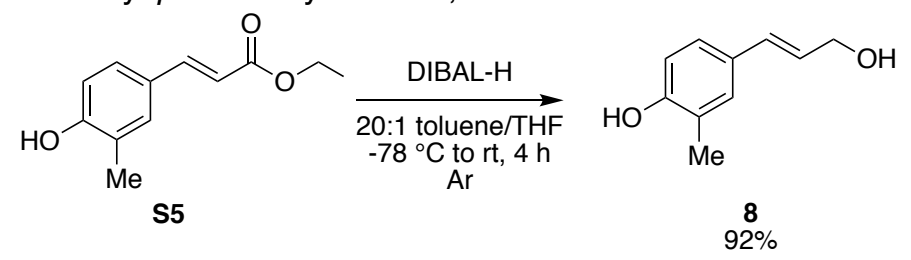

Compound $\mathbf{8}$ was prepared by DIBAL-H reduction of $\mathbf{S 5}$ into alcohol following similar protocols as $\mathbf{5}$ and purified by silica gel flash column chromatography eluting with $30 \% \mathrm{EtOAc/hexanes} \mathrm{to} \mathrm{yield} 8$ as a white powder (146.9 mg, 92.4\% yield). TLC (EtOAc/hexanes, 2:3 v/v): $\mathrm{R}_{\mathrm{f}}=0.26 .{ }^{1} \mathrm{H}$ NMR $\left(500 \mathrm{MHz}\right.$, Acetone- $\left.d_{6}\right)$ $\delta 8.27(\mathrm{~s}, 1 \mathrm{H}), 7.17(\mathrm{~d}, J=2.1 \mathrm{~Hz}, 1 \mathrm{H}), 7.07(\mathrm{dd}, J=8.2,2.1 \mathrm{~Hz}, 1 \mathrm{H}), 6.76(\mathrm{~d}, J=8.2 \mathrm{~Hz}, 1 \mathrm{H}), 6.47(\mathrm{dt}, J$ $=15.9,1.6 \mathrm{~Hz}, 1 \mathrm{H}), 6.18(\mathrm{dt}, J=15.9,5.5 \mathrm{~Hz}, 1 \mathrm{H}), 4.18($ app t, $J=5.5 \mathrm{~Hz}, 1 \mathrm{H}), 3.80(\mathrm{t}, J=5.5 \mathrm{~Hz}, 2 \mathrm{H})$, $2.18(\mathrm{~s}, 3 \mathrm{H}) .{ }^{13} \mathrm{C}$ NMR $\left(126 \mathrm{MHz}\right.$, Acetone- $\left.d_{6}\right) \delta 155.8,130.4,129.7,129.6,127.5,125.8,125.0,115.5$, 63.5, 16.2. HRMS (m/z): $\left[\mathrm{M}-\mathrm{H}_{2} \mathrm{O}+\mathrm{H}\right]^{+}$calcd. for $\mathrm{C}_{10} \mathrm{H}_{11} \mathrm{O}^{+}, 147.0804$; found, 147.0811. ${ }^{1} \mathrm{H}$ NMR spectroscopic data were consistent with reported literature values. ${ }^{5}$

3,5-dimethyl p-coumaryl alcohol, 9

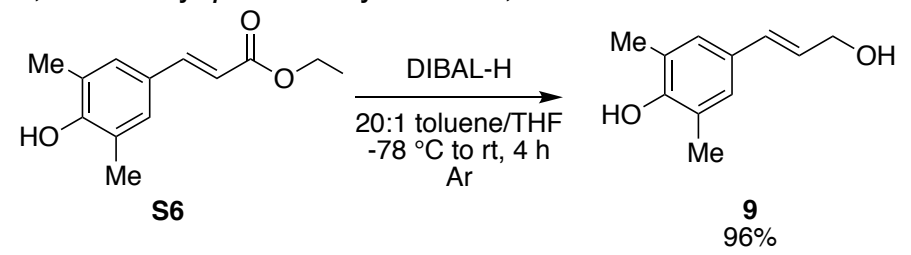

Compound $\mathbf{9}$ was prepared by DIBAL-H reduction of $\mathbf{S} \mathbf{6}$ into alcohol following similar protocols as $\mathbf{5}$ and purified by silica gel flash column chromatography eluting with $20 \%$ EtOAc/hexanes to yield $\mathbf{9}$ as a paleyellow powder (155.4 mg, 95.9\% yield). TLC (EtOAc/hexanes, 2:3 v/v): $R_{f}=0.34 .{ }^{1} \mathrm{H}$ NMR $(600 \mathrm{MHz}$, Acetone- $\left.d_{6}\right) \delta 7.27(\mathrm{~s}, 1 \mathrm{H}), 7.01(\mathrm{~s}, 2 \mathrm{H}), 6.44(\mathrm{dt}, J=15.9,1.7 \mathrm{~Hz}, 1 \mathrm{H}), 6.18(\mathrm{dt}, J=15.9,5.7 \mathrm{~Hz}, 1 \mathrm{H}), 4.18$ (td, $J=5.7,1.7 \mathrm{~Hz}, 2 \mathrm{H}), 3.73(\mathrm{t}, J=5.7 \mathrm{~Hz}, 1 \mathrm{H}), 2.21(\mathrm{~s}, 6 \mathrm{H}) .{ }^{13} \mathrm{C}$ NMR $\left(151 \mathrm{MHz}\right.$, Acetone- $\left.d_{6}\right) \delta 153.8$, 130.5, 129.7, 127.6, 127.4, 124.8, 63.6, 16.6. HRMS (m/z): $\left[\mathrm{M}-\mathrm{H}_{2} \mathrm{O}+\mathrm{H}\right]^{+}$calcd. for $\mathrm{C}_{11} \mathrm{H}_{13} \mathrm{O}^{+}, 161.0961$; found, 161.0952. ${ }^{1} \mathrm{H}$ NMR spectroscopic data were consistent with reported literature values. ${ }^{5}$

3-fluoro coniferyl alcohol, 10

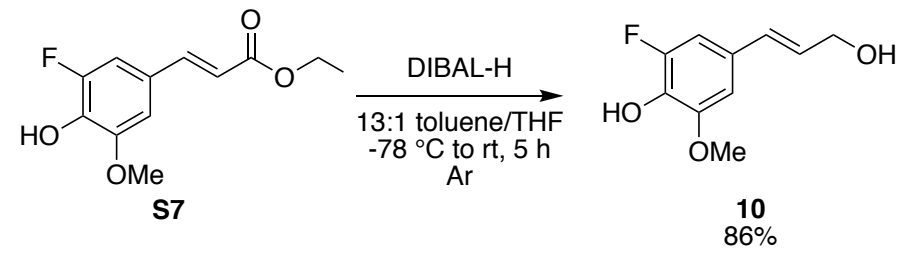

Compound $\mathbf{1 0}$ was prepared by DIBAL-H reduction of $\mathbf{S 7}$ into alcohol following similar protocols as $\mathbf{5}$ to yield 10 as a light-yellow powder $\left(141.2 \mathrm{mg}, 86 \%\right.$ yield). ${ }^{1} \mathrm{H}$ NMR $\left(400 \mathrm{MHz}\right.$, Acetone- $\left.d_{6}\right) \delta 8.14(\mathrm{~s}, 1 \mathrm{H})$, $6.86(\operatorname{app~t}, J=1.7 \mathrm{~Hz}, 1 \mathrm{H}), 6.81(\mathrm{dd}, J=11.5,1.7 \mathrm{~Hz}, 1 \mathrm{H}), 6.49(\mathrm{~d}, J=15.9 \mathrm{~Hz}, 1 \mathrm{H}), 6.29(\mathrm{dt}, J=15.9$, $5.3 \mathrm{~Hz}, 1 \mathrm{H}), 4.23(\mathrm{~d}, J=5.3 \mathrm{~Hz}, 2 \mathrm{H}), 4.18(\mathrm{~s}, 1 \mathrm{H}), 3.86(\mathrm{~s}, 3 \mathrm{H}) .{ }^{19} \mathrm{~F}$ NMR $\left(376 \mathrm{MHz}\right.$, Acetone- $\left.d_{6}\right) \delta-138.53$ (app d, $J=11.5 \mathrm{~Hz}) .{ }^{13} \mathrm{C}$ NMR $\left(126 \mathrm{MHz}\right.$, Acetone- $\left.d_{6}\right) \delta 152.2(\mathrm{~d}, J=238.4 \mathrm{~Hz}), 150.2(\mathrm{~d}, J=6.5 \mathrm{~Hz})$, 134.9 (d, $J=13.9 \mathrm{~Hz}), 129.8,129.5(\mathrm{~d}, J=8.5 \mathrm{~Hz}), 129.3$ (d, $J=2.5 \mathrm{~Hz}), 107.4(\mathrm{~d}, J=19.4 \mathrm{~Hz}), 106.1$ (d, $J=1.5 \mathrm{~Hz}), 63.1,56.7$. HRMS (m/z): $\left[\mathrm{M}-\mathrm{H}_{2} \mathrm{O}+\mathrm{H}\right]^{+}$calcd. for $\mathrm{C}_{10} \mathrm{H}_{10} \mathrm{FO}_{2}{ }^{+}, 181.0659$; found, 181.0657 .

3-ethoxy p-coumaryl alcohol, 11

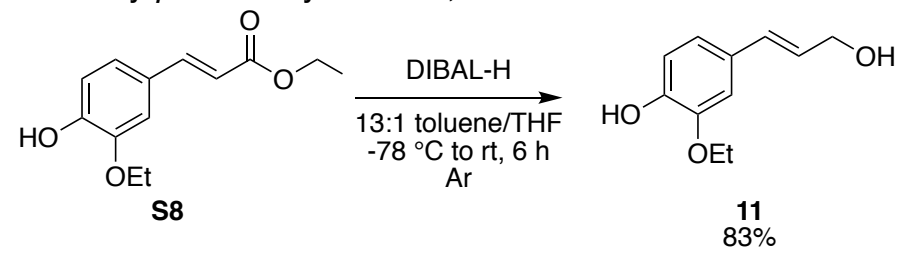


Compound $\mathbf{1 1}$ was prepared by DIBAL-H reduction of $\mathbf{S 8}$ into alcohol following similar protocols as $\mathbf{5}$ to give 11 as a light-yellow powder $\left(136.6 \mathrm{mg}, 83.3 \%\right.$ yield). ${ }^{1} \mathrm{H}$ NMR $\left(400 \mathrm{MHz}\right.$, Acetone- $\left.d_{6}\right) \delta 7.61(\mathrm{~s}, 1 \mathrm{H})$, $7.04(\mathrm{~d}, J=2.0 \mathrm{~Hz}, 1 \mathrm{H}), 6.85(\mathrm{dd}, J=8.2,2.0 \mathrm{~Hz}, 1 \mathrm{H}), 6.76(\mathrm{~d}, J=8.2 \mathrm{~Hz}, 1 \mathrm{H}), 6.48(\mathrm{dt}, J=15.9,1.7 \mathrm{~Hz}$, $1 \mathrm{H}), 6.21$ (dt, $J=15.9,5.6 \mathrm{~Hz}, 1 \mathrm{H}), 4.18(\mathrm{td}, J=5.6,1.6 \mathrm{~Hz}, 2 \mathrm{H}), 4.12(\mathrm{q}, J=7.0 \mathrm{~Hz}, 2 \mathrm{H}), 3.77(\mathrm{t}, J=5.6$ $\mathrm{Hz}, 1 \mathrm{H}), 1.37(\mathrm{t}, J=7.0 \mathrm{~Hz}, 3 \mathrm{H}) .{ }^{13} \mathrm{C}$ NMR $\left(126 \mathrm{MHz}\right.$, Acetone- $\left.d_{6}\right) \delta 147.6,147.3,130.4,130.2,128.1$, 120.6, 115.8, 111.0, 65.0, 63.4, 15.1. HRMS (m/z): $\left[\mathrm{M}-\mathrm{H}_{2} \mathrm{O}+\mathrm{H}\right]^{+}$calcd. for $\mathrm{C}_{11} \mathrm{H}_{13} \mathrm{O}_{2}{ }^{+}, 177.0910$; found, 177.0908. All spectroscopic data were consistent with reported literature values. ${ }^{5}$

\section{8-8' coupling products}

$( \pm)$-pinoresinols, 2 b

$( \pm)$-dehydrodiconiferyl alcohols, 2c
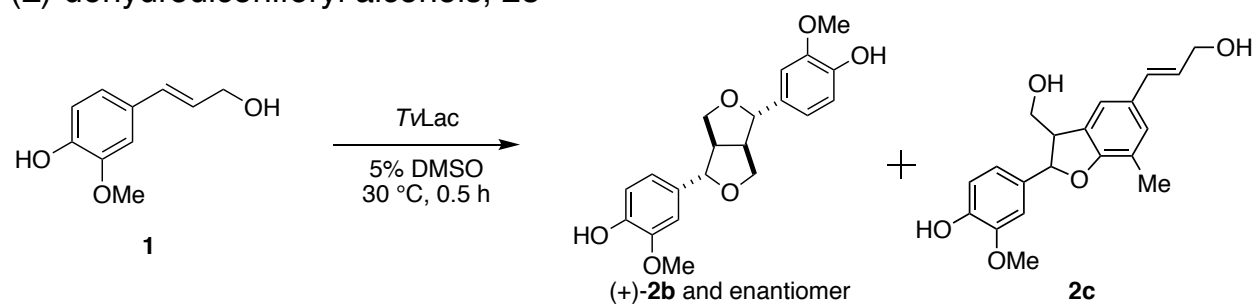

TVLac (1667 $\mu \mathrm{l}, 5 \mathrm{mU} / \mu \mathrm{l}$ in water) and MES buffer stock solution (2.5 ml, $1 \mathrm{M} \mathrm{MES,} \mathrm{pH} \mathrm{5.5)} \mathrm{were} \mathrm{diluted}$ with $19.6 \mathrm{ml}$ of deionized water. The reaction was initiated by addition of coniferyl alcohol 1 (200 mM in DMSO, $0.25 \mathrm{mmol}, 2$ equiv) and was shaken at $300 \mathrm{rpm}$ at $30^{\circ} \mathrm{C}$ for $0.5 \mathrm{~h}$. The aqueous reaction mixture was extracted three times with ethyl acetate right away to prevent further oxidation of the coupling products. Combined organic layers were dried over anhydrous sodium sulfate and concentrated in vacuo. The residue was resuspended in minimal volume of methanol and acetonitrile for prep HPLC purification to give, in the order of elution, $\mathbf{2 c}(1.0 \mathrm{mg}, 2.2 \%$ yield) and $\mathbf{2 b}(1.1 \mathrm{mg}, 2.5 \%$ yield).

$(+)-2 b /(-)-2 b:{ }^{1} \mathrm{H}$ NMR $\left(600 \mathrm{MHz}\right.$, Acetone- $\left.d_{6}\right) \delta 7.48(\mathrm{~s}, 2 \mathrm{H}), 6.99(\mathrm{~d}, J=1.9 \mathrm{~Hz}, 2 \mathrm{H}), 6.84(\mathrm{dd}, J=8.1,1.9$ $\mathrm{Hz}, 2 \mathrm{H}), 6.79(\mathrm{~d}, J=8.1 \mathrm{~Hz}, 2 \mathrm{H}), 4.67(\mathrm{~d}, J=4.2 \mathrm{~Hz}, 2 \mathrm{H}), 4.23-4.17(\mathrm{~m}, 2 \mathrm{H}), 3.85(\mathrm{~s}, 6 \mathrm{H}), 3.80(\mathrm{dd}, J=$ 9.1, 3.9 Hz, $2 \mathrm{H}), 3.11-3.07(\mathrm{~m}, 2 \mathrm{H})$. HRMS $(\mathrm{m} / \mathrm{z})$ : $\left[\mathrm{M}-\mathrm{H}_{2} \mathrm{O}+\mathrm{H}\right]^{+}$calcd. for $\mathrm{C}_{20} \mathrm{H}_{21} \mathrm{O}_{5}{ }^{+}, 341.1384$; found, 341.1384. All spectroscopic data were consistent with reported literature values. ${ }^{6}$

Note: in the structural representations of the racemate $( \pm)$-pinoresinols and their analogues, we have used bold bonds ( - ) and hashed bonds (....$\cdot)$ to denote the relative configurations of the enantiomers. The enantiomeric or enantioenriched products, for which stereochemistry was determined based on analogy with $(+)-\mathbf{2 b}$, are represented with solid wedges $(-)$ and hashed wedges $(\cdots+\cdot)$.

2c: ${ }^{1} \mathrm{H}$ NMR $\left(500 \mathrm{MHz}\right.$, Acetone- $\left.d_{6}\right) \delta 7.62(\mathrm{br} \mathrm{s}, 1 \mathrm{H}), 7.04(\mathrm{~d}, J=2.1 \mathrm{~Hz}, 1 \mathrm{H}), 6.98(\mathrm{~s}, 1 \mathrm{H}), 6.95(\mathrm{~s}, 1 \mathrm{H})$, $6.88(\mathrm{dd}, J=8.2,2.1 \mathrm{~Hz}, 1 \mathrm{H}), 6.81(\mathrm{~d}, J=8.2 \mathrm{~Hz}, 1 \mathrm{H}), 6.53($ app d, $J=16.0 \mathrm{~Hz}, 1 \mathrm{H}), 6.24(\mathrm{dt}, J=16.0,5.5$ $\mathrm{Hz}, 1 \mathrm{H}$ ), $5.56(\mathrm{~d}, J=6.3 \mathrm{~Hz}, 1 \mathrm{H}), 4.20(\operatorname{app~d}, J=5.4 \mathrm{~Hz}, 2 \mathrm{H}), 4.12$ (app s, 1H), $3.86(\mathrm{~s}, 3 \mathrm{H}), 3.91-3.79$ $(\mathrm{m}, 2 \mathrm{H}), 3.82(\mathrm{~s}, 3 \mathrm{H}), 3.53(\mathrm{q}, J=6.3 \mathrm{~Hz}, 1 \mathrm{H}) .{ }^{13} \mathrm{C} \mathrm{NMR}\left(126 \mathrm{MHz}\right.$, Acetone- $\left.d_{6}\right) \delta 149.0,148.3,147.3$, 145.2, 134.4, 131.9, 130.5, 130.4, 128.4, 119.6, 116.1, 115.7, 111.6, 110.5, 88.5, 64.6, 63.4, 56.4, 56.3, 54.8. HRMS (m/z): $\left[\mathrm{M}-\mathrm{H}_{2} \mathrm{O}+\mathrm{H}\right]^{+}$calcd. for $\mathrm{C}_{20} \mathrm{H}_{21} \mathrm{O}_{5}{ }^{+}$, 341.1384; found, 341.1373. ${ }^{1} \mathrm{H}$ NMR spectroscopic data were consistent with reported literature values. ${ }^{6}$

( \pm )-demethoxypinoresinols, $\mathbf{1 2 b}$
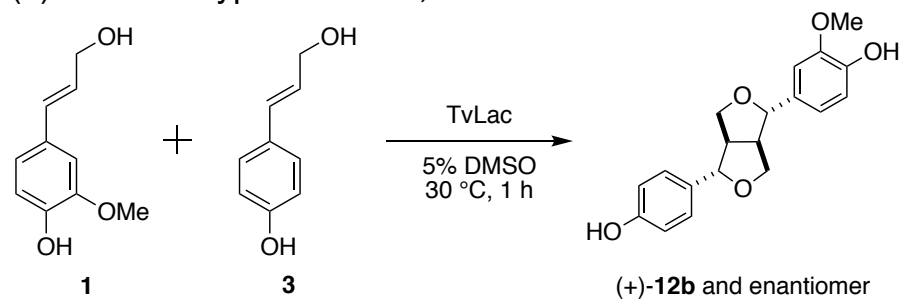

(+)-12b and enantiomer

Racemic demethoxypinoresinols (12b) were prepared by TVLac oxidation of coniferyl alcohol 1 (200 mM in DMSO, $0.05 \mathrm{mmol}, 1$ equiv) and p-coumaryl alcohol 3 (1 M in DMSO, $0.25 \mathrm{mmol}, 5$ equiv) following similar protocols as $\mathbf{2 b}$. The reaction crude concentrated in vacuo following ethyl acetate extraction was first purified by silica gel column chromatography (Biotage Selekt with Sfär silica HC Duo $20 \mu \mathrm{m} 5 \mathrm{~g}$ ) to separate 
regioisomers (mainly, 8-8' coupling products from 8-5' and 8-0-4' coupling products) with the following gradient: 0-2 column volume (CV), 10-30\% EtOAc/Hexanes; 2-7 CV, 30-40\% EtOAc/Hexanes; 7-10 CV, 40-100\% EtOAc/Hexanes; and 10-20 CV, 100\% EtOAc. The fractions containing 8-8' coupling products were concentrated in vacuo and resuspended in minimal volume of methanol and acetonitrile for masstriggered prep HPLC purification to give racemic $12 \mathrm{~b}\left(0.1 \mathrm{mg}, 0.6 \%\right.$ yield). ${ }^{1} \mathrm{H} \mathrm{NMR}\left(600 \mathrm{MHz}\right.$, Acetone- $\left.d_{6}\right)$ $\delta 8.27(\mathrm{~s}, 1 \mathrm{H}), 7.49(\mathrm{~s}, 1 \mathrm{H}), 7.22(\mathrm{~d}, J=8.6 \mathrm{~Hz}, 2 \mathrm{H}), 7.00(\mathrm{~s}, 1 \mathrm{H}), 6.86-6.77(\mathrm{~m}, 4 \mathrm{H}), 4.67(\mathrm{~d}, J=4.8 \mathrm{~Hz}$, $2 \mathrm{H}), 4.19(\mathrm{q}, J=8.6 \mathrm{~Hz}, 2 \mathrm{H}), 3.85(\mathrm{~s}, 3 \mathrm{H}), 3.82-3.77(\mathrm{~m}, 2 \mathrm{H}), 3.12-3.04(\mathrm{~m}, 2 \mathrm{H}) . \mathrm{HRMS}(\mathrm{m} / \mathrm{z})$ : [M$\left.\mathrm{H}_{2} \mathrm{O}+\mathrm{H}\right]^{+}$calcd. for $\mathrm{C}_{19} \mathrm{H}_{19} \mathrm{O}_{4}{ }^{+}, 311.1278$; found, 311.1255 .

$( \pm)$-medioresinol, 13b

$( \pm$ )-syringaresinol, 22b
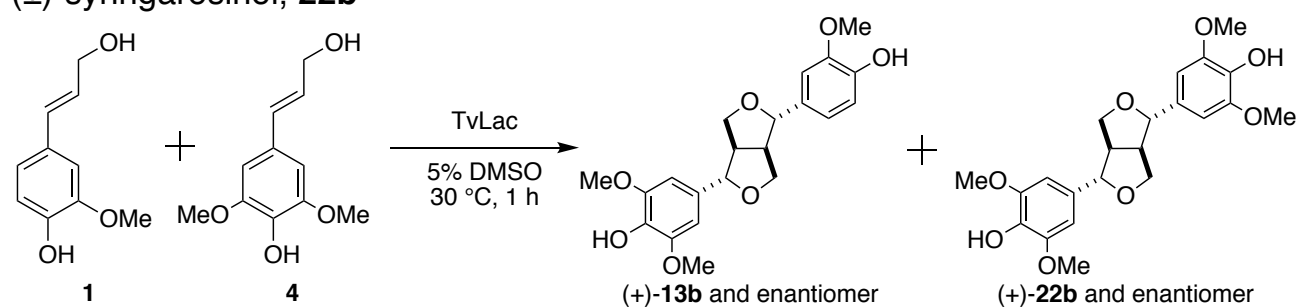

Racemic medioresinols (13b) and syringaresinols (22b) were prepared by T L Lac oxidation of coniferyl alcohol 1 (1 M in DMSO, 0.5 mmol, 5 equiv) and sinapyl alcohol 4 (200 mM in DMSO, 0.1 mmol, 1 equiv) following similar protocols as $\mathbf{1 2 b}$ to give, in the order of elution on C18 chromatography, racemic $\mathbf{2 2 b}$ (6.4 $\mathrm{mg}, 15.3 \%$ yield) and racemic $13 \mathrm{~b}(0.3 \mathrm{mg}, 0.8 \%$ yield $)$.

$(+)-13 \mathrm{~b} /(-)-13 \mathrm{~b}:{ }^{1} \mathrm{H}$ NMR $\left(600 \mathrm{MHz}\right.$, Acetone- $\left.d_{6}\right) \delta 7.48(\mathrm{~s}, 1 \mathrm{H}), 7.10(\mathrm{~s}, 1 \mathrm{H}), 6.99(\mathrm{~s}, 1 \mathrm{H}), 6.84(\mathrm{~d}, J=8.0$ $\mathrm{Hz}, 1 \mathrm{H}), 6.79(\mathrm{~d}, J=8.0 \mathrm{~Hz}, 1 \mathrm{H}), 6.69(\mathrm{~s}, 2 \mathrm{H}), 4.67(\mathrm{~d}, J=4.7 \mathrm{~Hz}, 2 \mathrm{H}), 4.25-4.18(\mathrm{~m}, 2 \mathrm{H}), 3.88-3.81$ $(\mathrm{m}, 2 \mathrm{H}), 3.84(\mathrm{~s}, 3 \mathrm{H}), 3.82(\mathrm{~s}, 6 \mathrm{H}), 3.13-3.06(\mathrm{~m}, 2 \mathrm{H})$. HRMS (m/z): $\left[\mathrm{M}-\mathrm{H}_{2} \mathrm{O}+\mathrm{H}\right]^{+}$calcd. for $\mathrm{C}_{21} \mathrm{H}_{23} \mathrm{O}_{6}{ }^{+}$, 371.1489; found, 371.1508. All spectroscopic data were consistent with reported literature values. ${ }^{7}$

(+)-22b/(-)-22b: ${ }^{1} \mathrm{H}$ NMR (500 MHz, Acetone- $\left.d_{6}\right) \delta 7.11(\mathrm{~s}, 2 \mathrm{H}), 6.68(\mathrm{~s}, 4 \mathrm{H}), 4.67(\mathrm{~d}, J=3.9 \mathrm{~Hz}, 2 \mathrm{H}), 4.26$ $-4.19(\mathrm{~m}, 2 \mathrm{H}), 3.84(\mathrm{~d}, J=3.9 \mathrm{~Hz}, 2 \mathrm{H}), 3.82(\mathrm{~s}, 12 \mathrm{H}), 3.12-3.07(\mathrm{~m}, 2 \mathrm{H}) .{ }^{13} \mathrm{C}$ NMR $(126 \mathrm{MHz}$, Acetone$\left.d_{6}\right) \delta 148.7,136.2,133.3,104.5,86.8,72.4,56.7,55.3$. HRMS $(\mathrm{m} / \mathrm{z})$ : $\left[\mathrm{M}-\mathrm{H}_{2} \mathrm{O}+\mathrm{H}\right]^{+}$calcd. for $\mathrm{C}_{22} \mathrm{H}_{25} \mathrm{O}_{7}{ }^{+}, 401.1595$; found, 401.1579. All spectroscopic data were consistent with reported literature values.

4-((1 $\left.S^{\star}, 3 a R^{\star}, 4 S^{\star}, 6 a R^{\star}\right)$-4-(4-hydroxy-3-(trifluoromethoxy)phenyl)tetrahydro-1 $H, 3 H$-furo[3,4-c]furan-1-yl)2-methoxyphenol, 14b<smiles>COc1cc(/C=C/CO)ccc1O</smiles>

Racemic coupling products $14 \mathrm{~b}$ were prepared by TVLac oxidation of coniferyl alcohol 1 (200 mM in DMSO, $0.05 \mathrm{mmol}, 1$ equiv) and $\mathbf{5}$ (1 $\mathrm{M}$ in DMSO, $0.25 \mathrm{mmol}, 5$ equiv) following similar protocols as $\mathbf{1 2 b}$. For prep HPLC purification, an isocratic elution at $40 \% \mathrm{~B}$ was used to give $0.2 \mathrm{mg}$ ( $1 \%$ yield). ${ }^{1} \mathrm{H} \mathrm{NMR}(600 \mathrm{MHz}$, Acetone- $\left.d_{6}\right) \delta 8.92(\mathrm{~s}, 1 \mathrm{H}), 7.49(\mathrm{~s}, 1 \mathrm{H}), 7.31(\mathrm{~s}, 1 \mathrm{H}), 7.25(\mathrm{~d}, J=8.5 \mathrm{~Hz}, 1 \mathrm{H}), 7.05(\mathrm{~d}, J=8.5 \mathrm{~Hz}, 1 \mathrm{H}), 7.00$ (s, 1H), $6.84(\mathrm{~d}, J=8.2 \mathrm{~Hz}, 1 \mathrm{H}), 6.79(\mathrm{~d}, J=8.2 \mathrm{~Hz}, 1 \mathrm{H}), 4.74(\mathrm{~d}, J=4.8 \mathrm{~Hz}, 1 \mathrm{H}), 4.67(\mathrm{~d}, J=4.8 \mathrm{~Hz}, 1 \mathrm{H})$, $4.26-4.16(\mathrm{~m}, 2 \mathrm{H}), 3.84(\mathrm{~s}, 3 \mathrm{H}), 3.85-3.80(\mathrm{~m}, 2 \mathrm{H}), 3.13-3.07(\mathrm{~m}, 2 \mathrm{H}) .{ }^{19} \mathrm{~F}$ NMR $(470 \mathrm{MHz}$, Acetone$\left.d_{6}\right) \delta$-58.78. HRMS (m/z): [M- $\left.\mathrm{H}_{2} \mathrm{O}+\mathrm{H}\right]^{+}$calcd. for $\mathrm{C}_{20} \mathrm{H}_{18} \mathrm{~F}_{3} \mathrm{O}_{5}{ }^{+}$, 395.1101; found, 395.1092. 


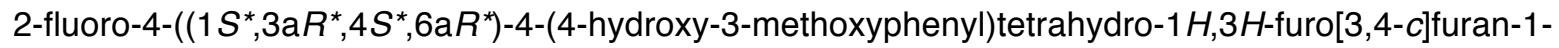
yl)phenol, 15b

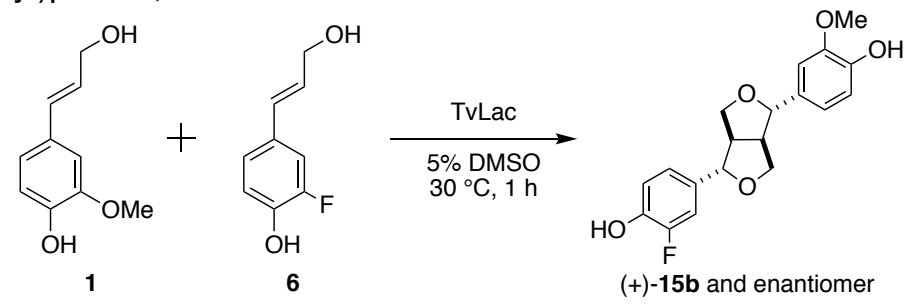

Racemic coupling products $15 \mathrm{~b}$ were prepared by TVLac oxidation of coniferyl alcohol 1 (200 mM in DMSO, $0.05 \mathrm{mmol}, 1$ equiv) and 6 (1 $\mathrm{M}$ in DMSO, $0.25 \mathrm{mmol}, 5$ equiv) following similar protocols as $12 \mathrm{~b}$ to give $0.4 \mathrm{mg}\left(2.3 \%\right.$ yield). ${ }^{1} \mathrm{H}$ NMR $\left(600 \mathrm{MHz}\right.$, Acetone- $\left.d_{6}\right) \delta 8.57(\mathrm{~s}, 1 \mathrm{H}), 7.49(\mathrm{~s}, 1 \mathrm{H}), 7.13(\mathrm{~d}, J=12.2 \mathrm{~Hz}, 1 \mathrm{H})$, $7.04(\mathrm{~d}, J=8.2 \mathrm{~Hz}, 1 \mathrm{H}), 6.99(\mathrm{~s}, 1 \mathrm{H}), 6.96(\operatorname{app~t}, J=8.8 \mathrm{~Hz}, 1 \mathrm{H}), 6.84(\mathrm{~d}, J=8.2 \mathrm{~Hz}, 1 \mathrm{H}), 6.79(\mathrm{~d}, J=8.2$ $\mathrm{Hz}, 1 \mathrm{H}), 4.70(\mathrm{~d}, J=4.4 \mathrm{~Hz}, 1 \mathrm{H}), 4.66(\mathrm{~d}, J=4.4 \mathrm{~Hz}, 1 \mathrm{H}), 4.23(\mathrm{t}, J=7.5 \mathrm{~Hz}, 1 \mathrm{H}), 4.18(\mathrm{t}, J=7.5 \mathrm{~Hz}, 1 \mathrm{H})$, $3.85(\mathrm{~s}, 3 \mathrm{H}), 3.84-3.80(\mathrm{~m}, 2 \mathrm{H}), 3.13-3.04(\mathrm{~m}, 2 \mathrm{H}) .{ }^{19} \mathrm{~F} \mathrm{NMR}\left(470 \mathrm{MHz}\right.$, Acetone- $\left.d_{6}\right) \delta-138.49(\mathrm{dd}, J=$ 12.2, $8.8 \mathrm{~Hz})$. HRMS (m/z): $\left[\mathrm{M}-\mathrm{H}_{2} \mathrm{O}+\mathrm{H}\right]^{+}$calcd. for $\mathrm{C}_{19} \mathrm{H}_{18} \mathrm{FO}_{4}{ }^{+}, 329.1184$; found, 329.1218 .

2-bromo-4-((1 $\left.S^{\star}, 3 a R^{*}, 4 S^{\star}, 6 \mathrm{a} R^{\star}\right)$-4-(4-hydroxy-3-methoxyphenyl)tetrahydro-1 $H, 3 H$-furo[3,4-c]furan-1yl)phenol, 16b

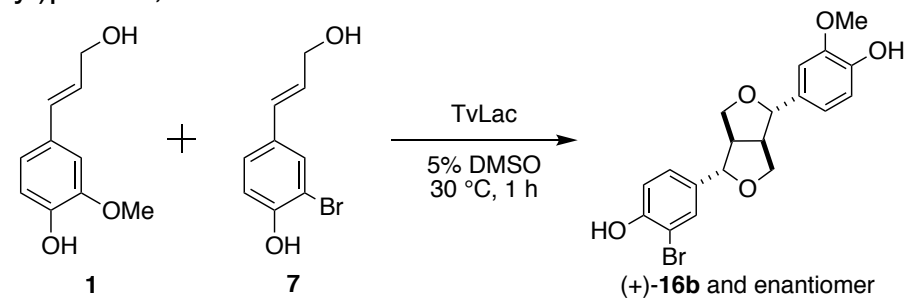

Racemic coupling products $16 \mathrm{~b}$ were prepared by TVLac oxidation of coniferyl alcohol 1 (200 mM in DMSO, $0.05 \mathrm{mmol}, 1$ equiv) and 7 (1 $\mathrm{M}$ in DMSO, $0.25 \mathrm{mmol}, 5$ equiv) following similar protocols as $\mathbf{1 2 b}$ to give $0.3 \mathrm{mg}\left(1.5 \%\right.$ yield). For prep HPLC purification, an isocratic elution at $35 \% \mathrm{~B}$ was used. ${ }^{1} \mathrm{H}$ NMR $(600 \mathrm{MHz}$, Acetone- $\left.d_{6}\right) \delta 8.86(\mathrm{~s}, 1 \mathrm{H}), 7.53(\mathrm{~s}, 1 \mathrm{H}), 7.49(\mathrm{~s}, 1 \mathrm{H}), 7.23(\mathrm{~d}, J=8.7 \mathrm{~Hz}, 1 \mathrm{H}), 7.01-6.96(\mathrm{~m}, 2 \mathrm{H}), 6.84(\mathrm{~d}$, $J=8.2 \mathrm{~Hz}, 1 \mathrm{H}), 6.79(\mathrm{~d}, J=8.2 \mathrm{~Hz}, 1 \mathrm{H}), 4.70(\mathrm{~d}, J=4.7 \mathrm{~Hz}, 1 \mathrm{H}), 4.67(\mathrm{~d}, J=4.7 \mathrm{~Hz}, 1 \mathrm{H}), 4.23(\mathrm{dd}, J=$ 9.3, $6.1 \mathrm{~Hz}, 1 \mathrm{H}$ ), $4.18(\mathrm{dd}, J=9.3,6.1 \mathrm{~Hz}, 1 \mathrm{H}), 3.84(\mathrm{~s}, 3 \mathrm{H}), 3.82(\mathrm{dd}, J=9.3,4.0 \mathrm{~Hz}, 2 \mathrm{H}), 3.14-3.05(\mathrm{~m}$, $2 \mathrm{H}$ ). HRMS (m/z): $\left[\mathrm{M}-\mathrm{H}_{2} \mathrm{O}+\mathrm{H}\right]^{+}$calcd. for $\mathrm{C}_{19} \mathrm{H}_{18} \mathrm{BrO}_{4}{ }^{+}, 389.0383$ (with ${ }^{79} \mathrm{Br}$ ) and 391.0363 (with ${ }^{81} \mathrm{Br}$ ); found, 389.0397 and 391.0358 , respectively. 
4-((1 $\left.S^{\star}, 3 \mathrm{a} R^{\star}, 4 S^{*}, 6 \mathrm{a} R^{\star}\right)$-4-(4-hydroxy-3-methoxyphenyl)tetrahydro-1 $H, 3 H$-furo[3,4-c]furan-1-yl)-2methylphenol, 17b

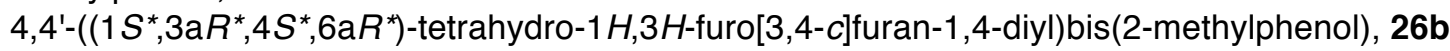
$(E)$-4-(3-(hydroxymethyl)-5-(3-hydroxyprop-1-en-1-yl)-7-methoxy-2,3-dihydrobenzofuran-2-yl)-2methylphenol, 17c

(E)-4-(3-(hydroxymethyl)-5-(3-hydroxyprop-1-en-1-yl)-7-methyl-2,3-dihydrobenzofuran-2-yl)-2methoxyphenol, 17c'

(E)-4-(3-(hydroxymethyl)-5-(3-hydroxyprop-1-en-1-yl)-7-methyl-2,3-dihydrobenzofuran-2-yl)-2methylphenol, 26c
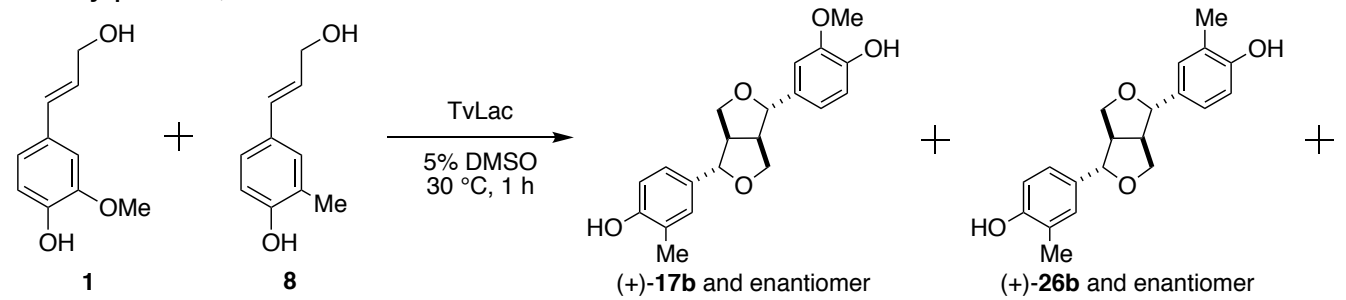

(+)-17b and enantiomer

(+)-26b and enantiomer
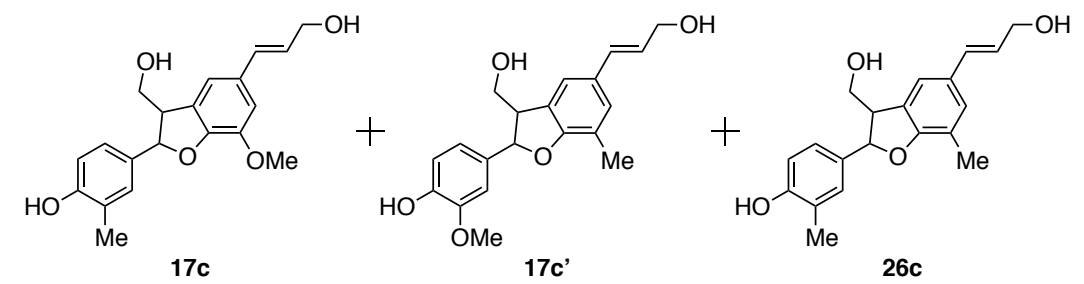

Racemic coupling products were prepared by TVLac oxidation of coniferyl alcohol 1 (200 mM in DMSO, $0.05 \mathrm{mmol}, 1$ equiv) and 8 (1 M in DMSO, $0.25 \mathrm{mmol}, 5$ equiv) following similar protocols as $12 \mathrm{~b}$. From the initial silica gel chromatography, the fractions containing 8-8' coupling products (17b and 26b) were combined, concentrated in vacuo, and purified by mass-triggered prep HPLC to give, in the order of elution, $17 \mathrm{~b}(0.3 \mathrm{mg}, 1.8 \%$ yield) and $\mathbf{2 6 b}(0.9 \mathrm{mg}, 5.5 \%$ yield). The fractions containing $8-5$ ' coupling products (17c, 17c', and 26c) were sequentially purified by mass-triggered prep HPLC to give, in the order of elution, 17c (0.6 mg, 3.5\% yield), 17c' (0.4 mg, $2.3 \%$ yield), and $26 \mathrm{c}(0.5 \mathrm{mg}, 3.1 \%$ yield).

$(+)-17 \mathrm{~b} /(-)-17 \mathrm{~b}:{ }^{1} \mathrm{H}$ NMR $\left(600 \mathrm{MHz}\right.$, Acetone- $\left.d_{6}\right) \delta 8.15(\mathrm{~s}, 1 \mathrm{H}), 7.50(\mathrm{~s}, 1 \mathrm{H}), 7.11(\mathrm{~s}, 1 \mathrm{H}), 7.03(\mathrm{~d}, J=8.2$ $\mathrm{Hz}, 1 \mathrm{H}), 6.99(\mathrm{~s}, 1 \mathrm{H}), 6.84(\mathrm{~d}, J=8.2 \mathrm{~Hz}, 1 \mathrm{H}), 6.78(\mathrm{app} \mathrm{dd}, J=8.2,4.5 \mathrm{~Hz}, 2 \mathrm{H}), 4.66(\mathrm{~d}, J=4.8 \mathrm{~Hz}, 1 \mathrm{H})$, $4.63(\mathrm{~d}, J=4.8 \mathrm{~Hz}, 1 \mathrm{H}), 4.22-4.15(\mathrm{~m}, 2 \mathrm{H}), 3.85(\mathrm{~s}, 3 \mathrm{H}), 3.78(\mathrm{dd}, J=9.0,4.1 \mathrm{~Hz}, 2 \mathrm{H}), 3.12-3.00(\mathrm{~m}$, $2 \mathrm{H}), 2.19(\mathrm{~s}, 3 \mathrm{H})$. HRMS (m/z): $\left[\mathrm{M}-\mathrm{H}_{2} \mathrm{O}+\mathrm{H}\right]^{+}$calcd. for $\mathrm{C}_{20} \mathrm{H}_{21} \mathrm{O}_{4}{ }^{+}, 325.1434$; found, 325.1456.

(+)-26b/(-)-26b: ${ }^{1} \mathrm{H}$ NMR (600 MHz, Acetone- $\left.d_{6}\right) \delta 8.12(\mathrm{~s}, 2 \mathrm{H}), 7.12(\mathrm{~s}, 2 \mathrm{H}), 7.03(\mathrm{~d}, J=8.2 \mathrm{~Hz}, 2 \mathrm{H}), 6.78$ (d, $J=8.2 \mathrm{~Hz}, 2 \mathrm{H}$ ), $4.62(\mathrm{~d}, J=3.5 \mathrm{~Hz}, 2 \mathrm{H}$ ), $4.19-4.15(\mathrm{~m}, 2 \mathrm{H}), 3.76$ (dd, $J=9.0,2.9 \mathrm{~Hz}, 2 \mathrm{H}$ ), $3.07-3.02$ (m, 2H), $2.19(\mathrm{~s}, 6 \mathrm{H})$. HRMS (m/z): [M- $\left.\mathrm{H}_{2} \mathrm{O}+\mathrm{H}\right]^{+}$calcd. for $\mathrm{C}_{20} \mathrm{H}_{21} \mathrm{O}_{3}{ }^{+}, 309.1485$; found, 309.1502 .

17c: ${ }^{1} \mathrm{H}$ NMR $\left(600 \mathrm{MHz}\right.$, Acetone- $\left.d_{6}\right) \delta 8.23(\mathrm{~s}, 1 \mathrm{H}), 7.15(\mathrm{~s}, 1 \mathrm{H}), 7.06(\mathrm{~d}, J=8.2 \mathrm{~Hz}, 1 \mathrm{H}), 6.98(\mathrm{~s}, 1 \mathrm{H})$, $6.94(\mathrm{~s}, 1 \mathrm{H}), 6.79(\mathrm{~d}, J=8.2 \mathrm{~Hz}, 1 \mathrm{H}), 6.52(\mathrm{~d}, J=15.9 \mathrm{~Hz}, 1 \mathrm{H}), 6.24(\mathrm{dt}, J=15.9,5.6 \mathrm{~Hz}, 1 \mathrm{H}), 5.53(\mathrm{~d}, J=$ $6.4 \mathrm{~Hz}, 1 \mathrm{H}), 4.20(\mathrm{t}, J=4.9 \mathrm{~Hz}, 2 \mathrm{H}), 4.09(\mathrm{~s}, 1 \mathrm{H}), 3.86(\mathrm{~s}, 3 \mathrm{H}), 3.84-3.78(\mathrm{~m}, 2 \mathrm{H}), 3.73(\mathrm{~s}, 1 \mathrm{H}), 3.50(\mathrm{q}, J$ $=6.3 \mathrm{~Hz}, 1 \mathrm{H}), 2.18(\mathrm{~s}, 3 \mathrm{H})$. HRMS $(\mathrm{m} / \mathrm{z}):\left[\mathrm{M}-\mathrm{H}_{2} \mathrm{O}+\mathrm{H}\right]^{+}$calcd. for $\mathrm{C}_{20} \mathrm{H}_{21} \mathrm{O}_{4}{ }^{+}, 325.1434$; found, 325.1454 .

17c': ${ }^{1} \mathrm{H}$ NMR $\left(600 \mathrm{MHz}\right.$, Acetone- $\left.d_{6}\right) \delta 7.57(\mathrm{~s}, 1 \mathrm{H}), 7.20(\mathrm{~s}, 1 \mathrm{H}), 7.14(\mathrm{~s}, 1 \mathrm{H}), 7.07-7.01(\mathrm{~m}, 2 \mathrm{H}), 6.88$ $(\mathrm{d}, J=8.3 \mathrm{~Hz}, 1 \mathrm{H}), 6.81(\mathrm{~d}, J=8.1 \mathrm{~Hz}, 1 \mathrm{H}), 6.51(\mathrm{~d}, J=15.8 \mathrm{~Hz}, 1 \mathrm{H}), 6.20(\mathrm{dt}, J=15.8,5.6 \mathrm{~Hz}, 1 \mathrm{H}), 5.55$ $(\mathrm{d}, J=6.4 \mathrm{~Hz}, 1 \mathrm{H}), 4.19(\mathrm{t}, J=5.3 \mathrm{~Hz}, 2 \mathrm{H}), 4.11(\mathrm{~s}, 1 \mathrm{H}), 3.88-3.84(\mathrm{~m}, 2 \mathrm{H}), 3.82(\mathrm{~s}, 3 \mathrm{H}), 3.70(\mathrm{~s}, 1 \mathrm{H})$, $3.55-3.48(\mathrm{~m}, 1 \mathrm{H}), 2.21(\mathrm{~s}, 3 \mathrm{H})$. HRMS (m/z): $\left[\mathrm{M}-\mathrm{H}_{2} \mathrm{O}+\mathrm{H}\right]^{+}$calcd. for $\mathrm{C}_{20} \mathrm{H}_{21} \mathrm{O}_{4}{ }^{+}, 325.1434$; found, 325.1453 . 26c: ${ }^{1} \mathrm{H}$ NMR $\left(600 \mathrm{MHz}\right.$, Acetone- $\left.d_{6}\right) \delta 8.22(\mathrm{~s}, 1 \mathrm{H}), 7.20(\mathrm{~s}, 1 \mathrm{H}), 7.14(\mathrm{~s}, 1 \mathrm{H}), 7.07-7.03(\mathrm{~m}, 2 \mathrm{H}), 6.79(\mathrm{~d}$, $J=8.2 \mathrm{~Hz}, 1 \mathrm{H}), 6.50(\mathrm{~d}, J=15.9 \mathrm{~Hz}, 1 \mathrm{H}), 6.20(\mathrm{dt}, J=15.9,5.6 \mathrm{~Hz}, 1 \mathrm{H}), 5.52(\mathrm{~d}, J=6.1 \mathrm{~Hz}, 1 \mathrm{H}), 4.19(\mathrm{t}$, $J=5.5 \mathrm{~Hz}, 2 \mathrm{H}), 4.09(\mathrm{~s}, 1 \mathrm{H}), 3.91-3.77(\mathrm{~m}, 2 \mathrm{H}), 3.70(\mathrm{~s}, 1 \mathrm{H}), 3.48(\mathrm{q}, J=6.1 \mathrm{~Hz}, 1 \mathrm{H}), 2.19(\operatorname{app~s}, 6 \mathrm{H})$. HRMS (m/z): $\left[\mathrm{M}-\mathrm{H}_{2} \mathrm{O}+\mathrm{H}\right]^{+}$calcd. for $\mathrm{C}_{20} \mathrm{H}_{21} \mathrm{O}_{3}{ }^{+}, 309.1485$; found, 309.1498. 
4-((1 $\left.S^{\star}, 3 a R^{\star}, 4 S^{\star}, 6 a R^{\star}\right)$-4-(4-hydroxy-3-methoxyphenyl)tetrahydro-1 $H, 3 H$-furo[3,4-c]furan-1-yl)-2,6dimethylphenol, 18b

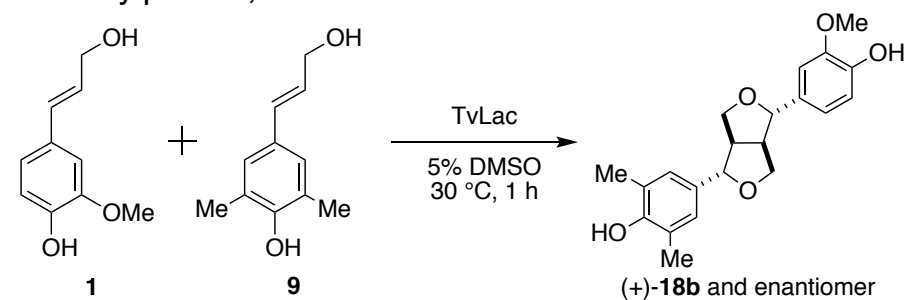

Racemic coupling products $18 \mathrm{~b}$ were prepared by TVLac oxidation of coniferyl alcohol 1 (200 mM in DMSO, $0.05 \mathrm{mmol}, 1$ equiv) and 9 (200 mM in DMSO, $0.25 \mathrm{mmol}, 1$ equiv) following similar protocols as $\mathbf{1 2 b}$ to give $0.4 \mathrm{mg}$ (2.2\% yield). For prep HPLC purification, an isocratic elution at $35 \% \mathrm{~B}$ was used. ${ }^{1} \mathrm{H}$ NMR (600 $\mathrm{MHz}$, Acetone- $\left.d_{6}\right) \delta 7.47(\mathrm{~s}, 1 \mathrm{H}), 7.18(\mathrm{~s}, 1 \mathrm{H}), 6.99(\mathrm{~d}, J=2.2 \mathrm{~Hz}, 1 \mathrm{H}), 6.96(\mathrm{~s}, 2 \mathrm{H}), 6.84(\mathrm{dd}, J=8.2,2.2$ $\mathrm{Hz}, 1 \mathrm{H}), 6.79(\mathrm{~d}, J=8.2 \mathrm{~Hz}, 1 \mathrm{H}), 4.66(\mathrm{~d}, J=4.7 \mathrm{~Hz}, 1 \mathrm{H}), 4.61(\mathrm{~d}, J=4.7 \mathrm{~Hz}, 1 \mathrm{H}), 4.22-4.15(\mathrm{~m}, 2 \mathrm{H})$, $3.85(\mathrm{~s}, 3 \mathrm{H}), 3.78(\mathrm{dd}, J=9.0,4.0 \mathrm{~Hz}, 2 \mathrm{H}), 3.11-3.01(\mathrm{~m}, 2 \mathrm{H}), 2.22(\mathrm{~s}, 6 \mathrm{H}) . \mathrm{HRMS}(\mathrm{m} / \mathrm{z}):\left[\mathrm{M}-\mathrm{H}_{2} \mathrm{O}+\mathrm{H}\right]^{+}$ calcd. for $\mathrm{C}_{21} \mathrm{H}_{23} \mathrm{O}_{4}{ }^{+}$, 339.1591; found, 339.1610.

2-fluoro-4-((1 $\left.S^{\star}, 3 a R^{\star}, 4 S^{\star}, 6 a R^{\star}\right)$-4-(4-hydroxy-3-methoxyphenyl)tetrahydro-1 $H, 3 H$-furo[3,4-c]furan-1-yl)-6methoxyphenol, 19b

(E)-2-fluoro-4-(3-(hydroxymethyl)-5-(3-hydroxyprop-1-en-1-yl)-7-methoxy-2,3-dihydrobenzofuran-2-yl)-6methoxyphenol, 19c

4,4'-((1 $\left.S^{\star}, 3 \mathrm{a} R^{*}, 4 S^{*}, 6 \mathrm{a} R^{*}\right)$-tetrahydro-1 $\mathrm{H}, 3 \mathrm{H}$-furo[3,4-c]furan-1,4-diyl)bis(2-fluoro-6-methoxyphenol), 28b
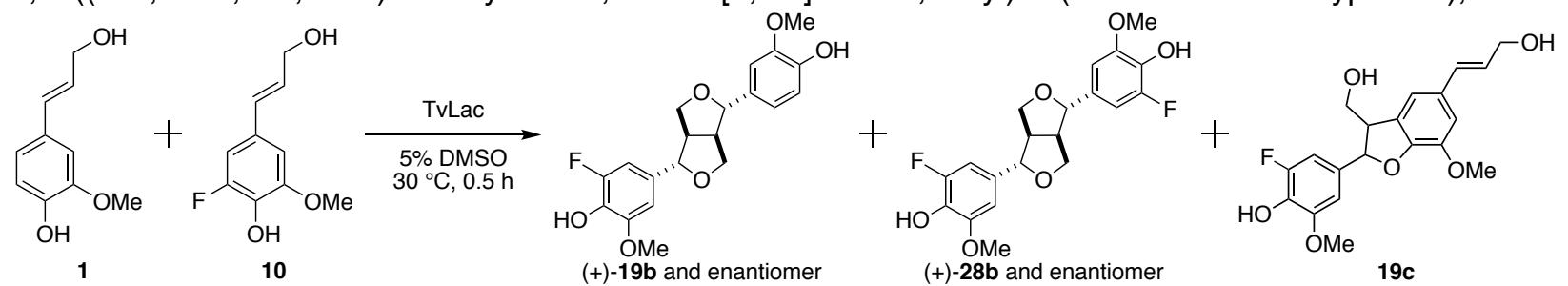

Racemic coupling products were prepared by TVLac oxidation of coniferyl alcohol 1 (200 mM in DMSO, $0.125 \mathrm{mmol}, 1$ equiv) and 10 (200 mM in DMSO, $0.125 \mathrm{mmol}, 1$ equiv) following similar protocols as $\mathbf{1 2 b}$. From the initial silica gel chromatography, the fractions containing 8-8' coupling products (19b and 28b) were purified by mass-triggered prep HPLC to give, in the order of elution, $19 \mathrm{~b}(0.7 \mathrm{mg}, 1.5 \%$ yield $)$ and 28b $(0.6 \mathrm{mg}, 1.2 \%$ yield). The fractions containing 8-5' coupling products (mainly $19 \mathrm{c}$ ) were further purified by mass-triggered prep HPLC to give $19 \mathrm{c}(0.7 \mathrm{mg}, 1.5 \%$ yield $)$.

$(+)-19 \mathrm{~b} /(-)-19 \mathrm{~b}:{ }^{1} \mathrm{H}$ NMR $\left(500 \mathrm{MHz}\right.$, Acetone- $\left.d_{6}\right) \delta 7.90(\mathrm{~s}, 1 \mathrm{H}), 7.54(\mathrm{~s}, 1 \mathrm{H}), 6.99(\mathrm{~d}, J=1.9 \mathrm{~Hz}, 1 \mathrm{H}), 6.87$ $-6.81(\mathrm{~m}, 2 \mathrm{H}), 6.81-6.75(\mathrm{~m}, 2 \mathrm{H}), 4.70(\mathrm{~d}, J=4.0 \mathrm{~Hz}, 1 \mathrm{H}), 4.66(\mathrm{~d}, J=4.3 \mathrm{~Hz}, 1 \mathrm{H}), 4.21(\mathrm{dd}, J=15.8$, $13.9 \mathrm{~Hz}, 2 \mathrm{H}), 3.87(\mathrm{~s}, 3 \mathrm{H}), 3.84(\mathrm{~s}, 3 \mathrm{H}), 3.84-3.80(\mathrm{~m}, 2 \mathrm{H}), 3.12-3.04(\mathrm{~m}, 2 \mathrm{H}) .{ }^{19} \mathrm{~F} \mathrm{NMR}(470 \mathrm{MHz}$, Acetone- $\left.d_{6}\right) \delta-138.36(\mathrm{~d}, J=11.0 \mathrm{~Hz})$. See compound $(+)-19 \mathrm{~b}$ for ${ }^{13} \mathrm{C}$ NMR. HRMS $(\mathrm{m} / \mathrm{z}):\left[\mathrm{M}-\mathrm{H}_{2} \mathrm{O}+\mathrm{H}\right]^{+}$ calcd. for $\mathrm{C}_{20} \mathrm{H}_{20} \mathrm{FO}_{5}{ }^{+}$, 359.1289; found, 359.1286.

(+)-28b/(-)-28b: ${ }^{1} \mathrm{H}$ NMR $\left(500 \mathrm{MHz}\right.$, Acetone- $\left.d_{6}\right) \delta 7.92(\mathrm{~s}, 2 \mathrm{H}), 6.85$ (app t, $\left.J=1.9 \mathrm{~Hz}, 2 \mathrm{H}\right), 6.78$ (dd, $J=$ $11.4,1.9 \mathrm{~Hz}, 2 \mathrm{H}), 4.69(\mathrm{~d}, J=3.9 \mathrm{~Hz}, 2 \mathrm{H}), 4.26-4.19(\mathrm{~m}, 2 \mathrm{H}), 3.87(\mathrm{~s}, 6 \mathrm{H}), 3.86-3.83(\mathrm{~m}, 2 \mathrm{H}), 3.12-$ $3.06(\mathrm{~m}, 2 \mathrm{H}) .{ }^{19} \mathrm{~F}$ NMR $\left(470 \mathrm{MHz}\right.$, Acetone- $\left.d_{6}\right) \delta-138.34($ app d, $J=11.4 \mathrm{~Hz}$ ). See compound $(+)-28 \mathrm{~b}$ for ${ }^{13} \mathrm{C}$ NMR. HRMS (m/z): [M- $\left.\mathrm{H}_{2} \mathrm{O}+\mathrm{H}\right]^{+}$calcd. for $\mathrm{C}_{20} \mathrm{H}_{19} \mathrm{~F}_{2} \mathrm{O}_{5}{ }^{+}, 377.1195$; found, 377.1195 .

19c: ${ }^{1} \mathrm{H}$ NMR $\left(500 \mathrm{MHz}\right.$, Acetone- $\left.d_{6}\right) \delta 8.03(\mathrm{~s}, 1 \mathrm{H}), 6.96(\mathrm{~s}, 2 \mathrm{H}), 6.91(\mathrm{t}, J=2.0 \mathrm{~Hz}, 1 \mathrm{H}), 6.81(\mathrm{dd}, J=11.4$, $2.0 \mathrm{~Hz}, 1 \mathrm{H}), 6.52(\mathrm{~d}, J=16.0 \mathrm{~Hz}, 1 \mathrm{H}), 6.25(\mathrm{dt}, J=16.0,5.5 \mathrm{~Hz}, 1 \mathrm{H}), 5.59(\mathrm{~d}, J=6.3 \mathrm{~Hz}, 1 \mathrm{H}), 4.19$ (app d, $J=4.5 \mathrm{~Hz}, 2 \mathrm{H}), 3.88(\mathrm{~s}, 3 \mathrm{H}), 3.85(\mathrm{~s}, 3 \mathrm{H}), 3.90-3.78(\mathrm{~m}, 2 \mathrm{H}), 3.77(\mathrm{~s}, 1 \mathrm{H}), 3.52(\mathrm{q}, J=6.3 \mathrm{~Hz}, 1 \mathrm{H}) .{ }^{19} \mathrm{~F}$ NMR $\left(470 \mathrm{MHz}\right.$, Acetone- $\left.d_{6}\right) \delta-137.74(\mathrm{~d}, J=11.4 \mathrm{~Hz}) \cdot{ }^{13} \mathrm{C}$ NMR $\left(126 \mathrm{MHz}\right.$, Acetone- $\left.d_{6}\right) \delta 152.3,152.0$ $(\mathrm{d}, J=239.7 \mathrm{~Hz}$ ), 150.3 (d, $J=6.2 \mathrm{~Hz}), 148.7,145.2,134.0$ (d, $J=8.0 \mathrm{~Hz}), 132.2,130.4,130.0,128.6$, 116.0, 111.7, 106.9 (d, $J=20.1 \mathrm{~Hz}), 106.0$ (d, $J=2.0 \mathrm{~Hz}), 87.9,64.7,63.4,56.8,56.4,55.0$. HRMS (m/z): $\left[\mathrm{M}-\mathrm{H}_{2} \mathrm{O}+\mathrm{H}\right]^{+}$calcd. for $\mathrm{C}_{20} \mathrm{H}_{20} \mathrm{FO}_{5}{ }^{+}, 359.1289$; found, 359.1278. 
2-ethoxy-4-((1 $\left.S^{*}, 3 \mathrm{a} R^{*}, 4 S^{*}, 6 \mathrm{a} R^{\star}\right)$-4-(4-hydroxy-3-methoxyphenyl)tetrahydro-1H,3H-furo[3,4-c]furan-1yl)phenol, 20b

4,4'-((1S*,3a $\left.R^{*}, 4 S^{*}, 6 \mathrm{a} R^{*}\right)$-tetrahydro-1 $H, 3 H$-furo[3,4-c]furan-1,4-diyl)bis(2-ethoxyphenol), 29b

(E)-2-ethoxy-4-(3-(hydroxymethyl)-5-(3-hydroxyprop-1-en-1-yl)-7-methoxy-2,3-dihydrobenzofuran-2-

yl)phenol, 20c

(E)-4-(7-ethoxy-3-(hydroxymethyl)-5-(3-hydroxyprop-1-en-1-yl)-2,3-dihydrobenzofuran-2-yl)-2methoxyphenol, 20c'

4,4'-((1S $\left., 3 a R^{\star}, 4 S^{\star}, 6 a R^{\star}\right)$-tetrahydro-1 $H, 3 H$-furo[3,4-c]furan-1,4-diyl)bis(2-ethoxyphenol), 29b

$(E)$-2-ethoxy-4-(7-ethoxy-3-(hydroxymethyl)-5-(3-hydroxyprop-1-en-1-yl)-2,3-dihydrobenzofuran-2-

yl)phenol, 29c

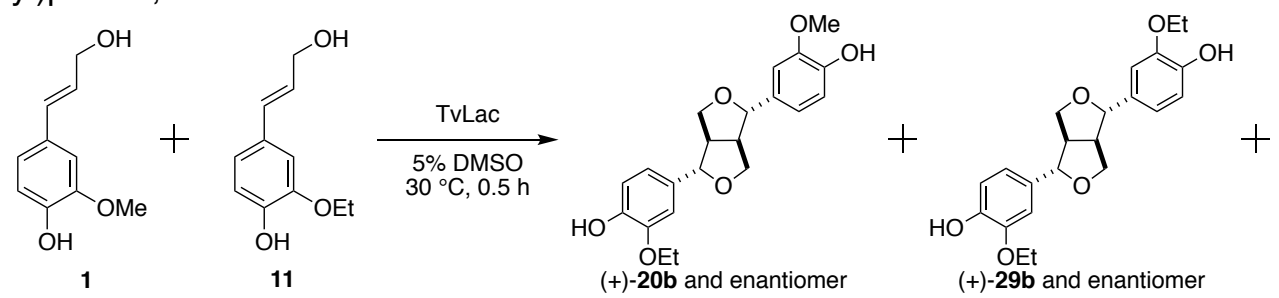

(+)-20b and enantiomer

(+)-29b and enantiomer

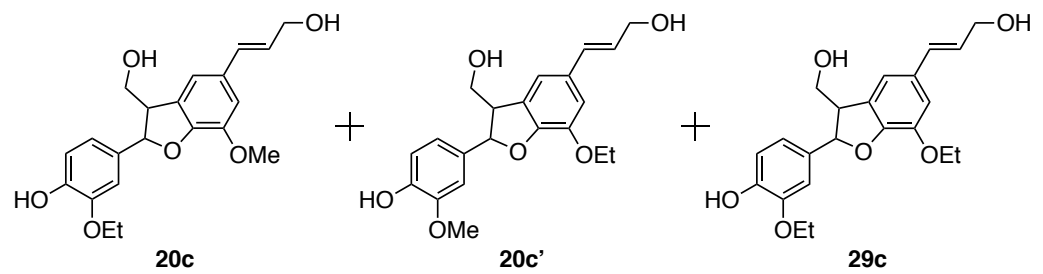

Racemic coupling products were prepared by TVLac oxidation of coniferyl alcohol 1 (200 mM in DMSO, $0.125 \mathrm{mmol}, 1$ equiv) and 11 (200 mM in DMSO, $0.125 \mathrm{mmol}, 1$ equiv) following similar protocols as $\mathbf{1 2 b}$. From the initial silica gel chromatography, the fractions containing 8-8' coupling products (20b and 29b) were purified by mass-triggered prep HPLC to give, in the order of elution, $20 \mathrm{~b}(0.6 \mathrm{mg}, 1.3 \%$ yield $)$ and $29 \mathrm{~b}$ (2.1 mg, 4.3\% yield). The fractions containing 8-5' coupling products $(20 \mathrm{c}, \mathbf{2 0 c}$ ', and $29 \mathrm{c})$ were further purified by mass-triggered prep HPLC to give a mixture of $20 \mathrm{c}$ and $20 \mathrm{c}^{\prime}(0.9 \mathrm{mg}, 1.9 \%$ yield) and $29 \mathrm{c}(2.9$ $\mathrm{mg}, 6.0 \%$ yield).

(+)-20b/(-)-20b: ${ }^{1} \mathrm{H}$ NMR $\left(600 \mathrm{MHz}\right.$, Acetone- $\left.d_{6}\right) \delta 7.49(\mathrm{~s}, 1 \mathrm{H}), 7.47(\mathrm{~s}, 1 \mathrm{H}), 6.99(\mathrm{~d}, J=1.9 \mathrm{~Hz}, 1 \mathrm{H}), 6.97$ $(\mathrm{d}, J=1.9 \mathrm{~Hz}, 1 \mathrm{H}), 6.86-6.80(\mathrm{~m}, 2 \mathrm{H}), 6.79(\operatorname{app~d}, J=8.1 \mathrm{~Hz}, 2 \mathrm{H}), 4.68-4.65(\mathrm{~m}, 1 \mathrm{H}), 4.23-4.16(\mathrm{~m}$, $1 \mathrm{H}), 4.10(\mathrm{q}, J=7.0 \mathrm{~Hz}, 1 \mathrm{H}), 3.84(\mathrm{~s}, 1 \mathrm{H}), 3.80(\mathrm{dd}, J=9.1,3.5 \mathrm{~Hz}, 1 \mathrm{H}), 3.11-3.05(\mathrm{~m}, 1 \mathrm{H}), 1.37(\mathrm{t}, J=$ 7.0 Hz, 1H). HRMS (m/z): [M- $\left.\mathrm{H}_{2} \mathrm{O}+\mathrm{H}\right]^{+}$calcd. for $\mathrm{C}_{21} \mathrm{H}_{23} \mathrm{O}_{5}{ }^{+}, 355.1540$; found, 355.1549.

(+)-29b/(-)-29b: ${ }^{1} \mathrm{H}$ NMR (600 MHz, Acetone- $\left.d_{6}\right) \delta 7.46(\mathrm{~s}, 2 \mathrm{H}), 6.97(\mathrm{~d}, J=1.9 \mathrm{~Hz}, 2 \mathrm{H}), 6.83$ (dd, $J=8.1$, $1.9 \mathrm{~Hz}, 2 \mathrm{H}), 6.78(\mathrm{~d}, J=8.1 \mathrm{~Hz}, 2 \mathrm{H}), 4.66(\mathrm{~d}, J=4.1 \mathrm{~Hz}, 2 \mathrm{H}), 4.21-4.17(\mathrm{~m}, 2 \mathrm{H}), 4.10(\mathrm{q}, J=7.0 \mathrm{~Hz}, 4 \mathrm{H})$, 3.79 (dd, $J=9.1,3.6 \mathrm{~Hz}, 2 \mathrm{H}), 3.10-3.05(\mathrm{~m}, 2 \mathrm{H}), 1.36(\mathrm{t}, J=7.0 \mathrm{~Hz}, 6 \mathrm{H})$. HRMS (m/z): $\left[\mathrm{M}-\mathrm{H}_{2} \mathrm{O}+\mathrm{H}\right]^{+}$calcd. for $\mathrm{C}_{22} \mathrm{H}_{25} \mathrm{O}_{5}{ }^{+}$, 369.1697; found, 369.1709.

20c/20c': 'H NMR (500 MHz, Acetone- $\left.d_{6}\right) \delta 7.61$ (s, 2H), 7.03 (app dd, $\left.J=11.4,2.3 \mathrm{~Hz}, 2 \mathrm{H}\right), 6.98(\mathrm{~s}, 2 \mathrm{H})$, $6.94($ app d, $J=10.9 \mathrm{~Hz}, 2 \mathrm{H}), 6.90-6.86(\mathrm{~m}, 2 \mathrm{H}), 6.81$ (app dd, $J=8.1,2.7 \mathrm{~Hz}, 2 \mathrm{H}), 6.52$ (app dd, $J=$ 15.9, $5.2 \mathrm{~Hz}, 2 \mathrm{H}), 6.24(\operatorname{app~dq}, J=15.9,5.4 \mathrm{~Hz}, 2 \mathrm{H}), 5.55(\mathrm{~d}, J=6.6 \mathrm{~Hz}, 2 \mathrm{H}), 4.19(\operatorname{app~d}, J=5.4 \mathrm{~Hz}, 4 \mathrm{H})$, $4.18-4.10(\mathrm{~m}, 2 \mathrm{H}), 4.13(\mathrm{q}, J=6.8 \mathrm{~Hz}, 2 \mathrm{H}), 4.06(\mathrm{q}, J=7.0 \mathrm{~Hz}, 2 \mathrm{H}), 3.94-3.83(\mathrm{~m}, 4 \mathrm{H}), 3.86(\mathrm{~s}, 3 \mathrm{H})$, $3.82(\mathrm{~s}, 3 \mathrm{H}), 3.76(\mathrm{~s}, 2 \mathrm{H}), 3.52(\mathrm{q}, J=6.2 \mathrm{~Hz}, 2 \mathrm{H}), 1.35$ (app td, $J=7.0,2.1 \mathrm{~Hz}, 6 \mathrm{H}) .{ }^{13} \mathrm{C}$ NMR $(126 \mathrm{MHz}$, Acetone- $\left.d_{6}\right) \delta 147.5,147.4,147.3,146.1,145.2,144.3,143.70,143.67,134.4,131.90,131.87,130.55$, $130.49,130.4,128.4,128.3,119.7,119.5,116.09,116.07,115.7,115.6,113.1,111.6,111.4,110.5,88.5$, $65.10,65.07,64.6,63.4,56.3,56.2,54.83,54.76,15.3,15.0$. (Downfield carbon peaks at $>140 \mathrm{ppm}$ are not well-resolved due to low yields and presence of two structurally similar regioisomers in the sample. See Figure S12c for MS/MS fragmentation pattern.) HRMS (m/z): $\left[\mathrm{M}-\mathrm{H}_{2} \mathrm{O}+\mathrm{H}\right]^{+}$calcd. for $\mathrm{C}_{21} \mathrm{H}_{23} \mathrm{O}_{5}{ }^{+}, 355.1540$; found, 355.1525.

29c: ${ }^{1} \mathrm{H}$ NMR $\left(500 \mathrm{MHz}\right.$, Acetone- $\left.d_{6}\right) \delta 7.57(\mathrm{~s}, 1 \mathrm{H}), 7.02(\mathrm{~d}, J=2.0 \mathrm{~Hz}, 1 \mathrm{H}), 6.98(\mathrm{~s}, 1 \mathrm{H}), 6.92(\mathrm{~d}, J=1.8$ $\mathrm{Hz}, 1 \mathrm{H}), 6.88(\mathrm{dd}, J=8.2,2.0 \mathrm{~Hz}, 1 \mathrm{H}), 6.81(\mathrm{~d}, J=8.2 \mathrm{~Hz}, 1 \mathrm{H}), 6.51(\mathrm{dt}, J=15.9,1.8 \mathrm{~Hz}, 1 \mathrm{H}), 6.23(\mathrm{dt}, J$ $=15.9,5.5 \mathrm{~Hz}, 1 \mathrm{H}), 5.55(\mathrm{~d}, J=6.6 \mathrm{~Hz}, 1 \mathrm{H}), 4.19(\mathrm{~d}, J=5.7 \mathrm{~Hz}, 2 \mathrm{H}), 4.16-4.12(\mathrm{~m}, 2 \mathrm{H}), 4.18-4.06(\mathrm{~m}$, 
$1 \mathrm{H}), 4.07(\mathrm{q}, J=7.0 \mathrm{~Hz}, 2 \mathrm{H}), 3.91-3.79(\mathrm{~m}, 2 \mathrm{H}), 3.73(\mathrm{~s}, 1 \mathrm{H}), 3.52(\mathrm{q}, J=5.7 \mathrm{~Hz}, 1 \mathrm{H}), 1.35(\mathrm{td}, J=7.0$, $3.8 \mathrm{~Hz}, 6 \mathrm{H}) .{ }^{13} \mathrm{C}$ NMR $\left(126 \mathrm{MHz}\right.$, Acetone- $\left.d_{6}\right) \delta 149.3,147.5,147.5,144.3,134.4,131.9,130.6,130.5$, $128.4,119.6,116.2,115.7,113.3,111.5,88.5,65.2,65.1,64.7,63.4,54.8,15.3,15.1$. HRMS (m/z): [M$\left.\mathrm{H}_{2} \mathrm{O}+\mathrm{H}\right]^{+}$calcd. for $\mathrm{C}_{22} \mathrm{H}_{25} \mathrm{O}_{5}{ }^{+}$, 369.1697; found, 369.1682.

(+)-pinoresinol, (+)-2b

2-fluoro-4-((1S,3aR,4S,6aR)-4-(4-hydroxy-3-methoxyphenyl)tetrahydro-1H,3H-furo[3,4-c]furan-1-yl)-6methoxyphenol, (+)-19b

4,4'-((1S,3aR,4S,6aR)-tetrahydro-1H,3H-furo[3,4-c]furan-1,4-diyl)bis(2-fluoro-6-methoxyphenol), (+)-28b

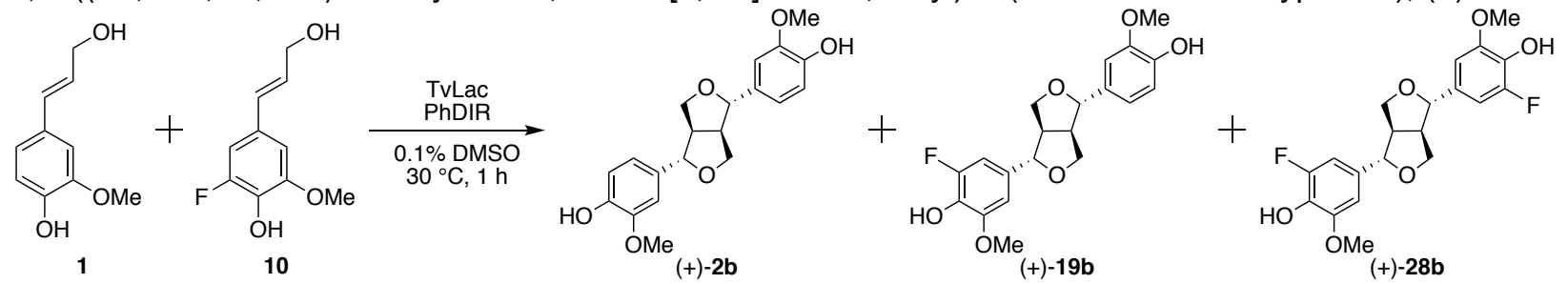

Plant extract containing PhDIR was diluted 10-fold to a final buffer concentration of $0.1 \mathrm{M}$ MES at pH 5.5, containing $0.3 \mathrm{M} \mathrm{NaCl}$ and $0.333 \mathrm{mU} / \mu \mathrm{l}$ TVLac. (While NaCl is a known laccase inhibitor, the present amount of $\mathrm{NaCl}$ did not halt the reaction. The addition of $\mathrm{NaCl}$ was necessary to slow down the undesired oxidation of the coupling products prior to extraction.) The reaction was initiated with the addition of equimolar mixture solution of 1 and 10 (1 M in DMSO, $17.5 \mu \mathrm{l}$ each) and placed at $30{ }^{\circ} \mathrm{C}$, shaking at 300 $\mathrm{rpm}$ for $1 \mathrm{~h}$. The reaction mixture was immediately extracted into ethyl acetate three times to prevent further oxidation of the coupling products by TVLac. Combined organic layers were dried over anhydrous sodium sulfate and concentrated in vacuo. The residue was resuspended in minimal volume of methanol and acetonitrile for prep HPLC injection to give, in the order of elution, $(+)-\mathbf{2 b}(3-9 \%$ yield), $(+)-\mathbf{1 9 b}(13-18 \%$ yield), and (+)-28b (12-19\% yield). Absolute stereochemistry was assigned based on analogy with $(+)-$ pinoresinol, (+)-2b. This protocol was repeated three times.

(+)-2b: ${ }^{1} \mathrm{H}$ NMR $\left(600 \mathrm{MHz}\right.$, Acetone- $\left.d_{6}\right) \delta 7.48(\mathrm{~s}, 2 \mathrm{H}), 6.99(\mathrm{~d}, J=1.9 \mathrm{~Hz}, 2 \mathrm{H}), 6.84$ (dd, $J=8.1,1.9 \mathrm{~Hz}$, 2H), $6.79(\mathrm{~d}, J=8.1 \mathrm{~Hz}, 2 \mathrm{H}), 4.67(\mathrm{~d}, J=4.2 \mathrm{~Hz}, 2 \mathrm{H}), 4.22-4.18(\mathrm{~m}, 2 \mathrm{H}), 3.85(\mathrm{~s}, 6 \mathrm{H}), 3.80(\mathrm{dd}, J=9.1$, $3.9 \mathrm{~Hz}, 2 \mathrm{H}), 3.10-3.07(\mathrm{~m}, 2 \mathrm{H})$. HRMS $(\mathrm{m} / \mathrm{z}):\left[\mathrm{M}-\mathrm{H}_{2} \mathrm{O}+\mathrm{H}\right]^{+}$calcd. for $\mathrm{C}_{20} \mathrm{H}_{21} \mathrm{O}_{5}{ }^{+}, 341.1384$; found, 341.1389 . All spectroscopic data were consistent with reported literature values. ${ }^{6}$

(+)-19b: ${ }^{1} \mathrm{H}$ NMR (500 MHz, Acetone- $\left.d_{6}\right) \delta 7.90(\mathrm{~s}, 1 \mathrm{H}), 7.54(\mathrm{~s}, 1 \mathrm{H}), 6.99(\mathrm{~d}, J=1.9 \mathrm{~Hz}, 1 \mathrm{H}), 6.87-6.80$ $(\mathrm{m}, 2 \mathrm{H}), 6.81-6.75(\mathrm{~m}, 2 \mathrm{H}), 4.70(\mathrm{~d}, J=4.0 \mathrm{~Hz}, 1 \mathrm{H}), 4.66(\mathrm{~d}, J=4.3 \mathrm{~Hz}, 1 \mathrm{H}), 4.26-4.16(\mathrm{~m}, 2 \mathrm{H}), 3.87$ $(\mathrm{s}, 3 \mathrm{H}), 3.84(\mathrm{~s}, 3 \mathrm{H}), 3.85-3.79(\mathrm{~m}, 2 \mathrm{H}), 3.13-3.04(\mathrm{~m}, 2 \mathrm{H}) .{ }^{19} \mathrm{~F}$ NMR $\left(470 \mathrm{MHz}\right.$, Acetone- $\left.d_{6}\right) \delta-138.37$ (d, $J=11.3 \mathrm{~Hz}) .{ }^{13} \mathrm{C}$ NMR $\left(126 \mathrm{MHz}\right.$, Acetone- $\left.d_{6}\right) \delta 152.0(\mathrm{~d}, J=238.4 \mathrm{~Hz}), 150.2(\mathrm{~d}, J=6.2 \mathrm{~Hz}), 148.3$, 146.9, $134.6(\mathrm{~d}, J=13.9 \mathrm{~Hz}), 134.0,133.8(\mathrm{~d}, J=7.4 \mathrm{~Hz}), 119.6,115.5,110.6,106.9(\mathrm{~d}, J=19.8 \mathrm{~Hz})$, $106.1(\mathrm{~d}, J=1.9 \mathrm{~Hz}), 86.6,86.0,72.3(2 \mathrm{C}), 56.7,56.2,55.3,55.1$. HRMS (m/z): $\left[\mathrm{M}-\mathrm{H}_{2} \mathrm{O}+\mathrm{H}\right]^{+}$calcd. for $\mathrm{C}_{20} \mathrm{H}_{20} \mathrm{FO}_{5}{ }^{+}$, 359.1289; found, 359.1288.

(+)-28b: ${ }^{1} \mathrm{H}$ NMR (500 MHz, Acetone- $\left.d_{6}\right) \delta 7.90(\mathrm{~s}, 2 \mathrm{H}), 6.84(\mathrm{t}, \mathrm{J}=1.9 \mathrm{~Hz}, 2 \mathrm{H}), 6.78$ (dd, J=11.4, $1.9 \mathrm{~Hz}$, 2H), $4.69(\mathrm{~d}, J=3.8 \mathrm{~Hz}, 2 \mathrm{H}), 4.26-4.19(\mathrm{~m}, 2 \mathrm{H}), 3.87(\mathrm{~s}, 6 \mathrm{H}), 3.86-3.83(\mathrm{~m}, 2 \mathrm{H}), 3.13-3.06(\mathrm{~m}, 2 \mathrm{H})$. ${ }^{19} \mathrm{~F}$ NMR $\left(470 \mathrm{MHz}\right.$, Acetone- $\left.d_{6}\right) \delta-138.36(\mathrm{~d}, J=11.4 \mathrm{~Hz}) .{ }^{13} \mathrm{C}$ NMR $(126 \mathrm{MHz}$, Acetone $) \delta 152.0(\mathrm{~d}, J=$ $239.1 \mathrm{~Hz}), 150.2(\mathrm{~d}, J=6.2 \mathrm{~Hz}), 134.6(\mathrm{~d}, J=13.8 \mathrm{~Hz}), 133.7(\mathrm{~d}, J=7.6 \mathrm{~Hz}), 107.0(\mathrm{~d}, J=19.8 \mathrm{~Hz}), 106.1$ (d, $J=1.9 \mathrm{~Hz}), 86.0,72.4,56.7,55.1$. HRMS (m/z): $\left[\mathrm{M}-\mathrm{H}_{2} \mathrm{O}+\mathrm{H}\right]^{+}$calcd. for $\mathrm{C}_{20} \mathrm{H}_{19} \mathrm{~F}_{2} \mathrm{O}_{5}{ }^{+}, 377.1195$; found, 377.1193. 
4-((1S,3aR,4S,6aR)-4-(4-hydroxy-3-methoxyphenyl)tetrahydro-1 $H, 3 H$-furo[3,4-c]furan-1-yl)-2methylphenol, $(+)-17 b$<smiles>COc1cc(/C=C/CO)ccc1O</smiles>

Enantioenriched coupling products were prepared by PhDIR-assisted TVLac oxidation reaction following similar protocols as (+)-2b. The reaction was initiated by the addition of a mixture of coniferyl alcohol 1 (200 $\mathrm{mM}$ in DMSO, $0.015 \mathrm{mmol}, 1$ equiv) and 8 (600 mM in DMSO, $0.045 \mathrm{mmol}, 3$ equiv). The reaction crude was purified following similar protocols as $\mathbf{1 2 b}$ to give $(+)-\mathbf{1 7 b}(0.2 \mathrm{mg}, 3.9 \%$ yield $)$ and $(+)-\mathbf{2 6 b}(0.3 \mathrm{mg}$, $6.1 \%$ yield). (Note: the homocoupling product $\mathbf{2 6 b}$ isolated from this preparatory reaction was only slightly enantioenriched with $8 \%$ ee (compared to the increase from $0 \%$ ee (EV) to $31 \%$ ee (PhDIR) at the analytical scale, see Figure $\mathbf{S 1 0}$ for chiral chromatography). Thus, this product was considered not significantly influenced by PhDIR-mediated coupling and excluded from reports in Table 3.) Absolute stereochemistry was assigned based on analogy with (+)-pinoresinol, (+)-2b.

(+)-17b: ' ${ }^{H} \mathrm{H}$ NMR $\left(600 \mathrm{MHz}\right.$, Acetone- $\left.d_{6}\right) \delta 8.12(\mathrm{~s}, 1 \mathrm{H}), 7.48(\mathrm{~s}, 1 \mathrm{H}), 7.11(\mathrm{~s}, 1 \mathrm{H}), 7.03(\mathrm{~d}, J=8.2 \mathrm{~Hz}, 1 \mathrm{H})$, $6.99(\mathrm{~s}, 1 \mathrm{H}), 6.83(\mathrm{~d}, J=8.2 \mathrm{~Hz}, 1 \mathrm{H}), 6.78(\operatorname{app~dd}, J=8.2,5.3 \mathrm{~Hz}, 2 \mathrm{H}), 4.66(\mathrm{~d}, J=4.8 \mathrm{~Hz}, 1 \mathrm{H}), 4.63(\mathrm{~d}$, $J=4.8 \mathrm{~Hz}, 1 \mathrm{H}), 4.22-4.15(\mathrm{~m}, 2 \mathrm{H}), 3.85(\mathrm{~s}, 3 \mathrm{H}), 3.78(\mathrm{dd}, J=9.0,4.1 \mathrm{~Hz}, 2 \mathrm{H}), 3.11-3.01(\mathrm{~m}, 2 \mathrm{H}), 2.19$ $(\mathrm{s}, 3 \mathrm{H})$. HRMS (m/z): $\left[\mathrm{M}-\mathrm{H}_{2} \mathrm{O}+\mathrm{H}\right]^{+}$calcd. for $\mathrm{C}_{20} \mathrm{H}_{21} \mathrm{O}_{4}{ }^{+}, 325.1434$; found, 325.1457.

(+)-26b: ${ }^{1} \mathrm{H}$ NMR $\left(600 \mathrm{MHz}\right.$, Acetone- $\left.d_{6}\right) \delta 8.13(\mathrm{~s}, 2 \mathrm{H}), 7.12(\mathrm{~s}, 2 \mathrm{H}), 7.03(\mathrm{~d}, J=8.2 \mathrm{~Hz}, 2 \mathrm{H}), 6.78(\mathrm{~d}, J=$ $8.2 \mathrm{~Hz}, 2 \mathrm{H}), 4.63(\mathrm{~d}, J=3.5 \mathrm{~Hz}, 2 \mathrm{H}), 4.19-4.15(\mathrm{~m}, 2 \mathrm{H}), 3.76(\mathrm{dd}, J=9.0,2.9 \mathrm{~Hz}, 2 \mathrm{H}), 3.07-3.03(\mathrm{~m}$, $2 \mathrm{H}), 2.19(\mathrm{~s}, 6 \mathrm{H})$. HRMS (m/z): $\left[\mathrm{M}-\mathrm{H}_{2} \mathrm{O}+\mathrm{H}\right]^{+}$calcd. for $\mathrm{C}_{20} \mathrm{H}_{21} \mathrm{O}_{3}{ }^{+}, 309.1485$; found, 309.1502.

2-ethoxy-4-((1S,3aR,4S,6aR)-4-(4-hydroxy-3-methoxyphenyl)tetrahydro-1H,3H-furo[3,4-c]furan-1yl)phenol, $(+)-20 b$

4,4'-((1S,3aR,4S,6aR)-tetrahydro-1H,3H-furo[3,4-c]furan-1,4-diyl)bis(2-ethoxyphenol), (+)-29b
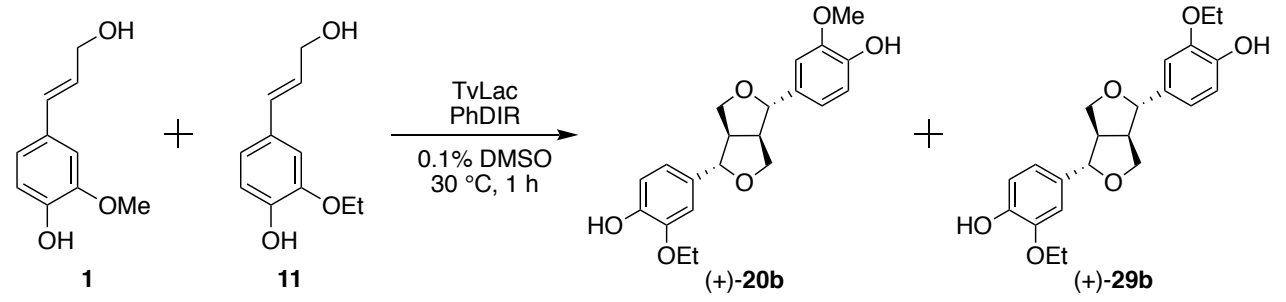

Enantioenriched coupling products were prepared by PhDIR-assisted TVLac oxidation reaction of $1 \mathrm{mM}$ equimolar mixture of $\mathbf{1}$ and $\mathbf{1 1}$ following similar protocols as (+)-2b. Following the ethyl acetate extraction, the concentrated residue was resuspended in minimal volume of methanol and acetonitrile for prep HPLC injection to give (+)-29b (9\% yield). The fraction containing (+)-20b and $29 \mathrm{c}$ was further purified by silica gel flash column chromatography with $30-40 \%$ EtOAc/hexanes followed by prep HPLC purification to give $(+)-20 b(4-9 \%$ yield). Absolute stereochemistry was assigned based on analogy with (+)-2b. This protocol was repeated twice, but the fraction containing (+)-29b in one of the trials was lost.

(+)-20b: ${ }^{1} \mathrm{H}$ NMR $\left(600 \mathrm{MHz}\right.$, Acetone- $\left.d_{6}\right) \delta 7.49(\mathrm{~s}, 1 \mathrm{H}), 7.47(\mathrm{~s}, 1 \mathrm{H}), 6.99(\mathrm{~d}, J=1.8 \mathrm{~Hz}, 1 \mathrm{H}), 6.97(\mathrm{~d}, J=$ $1.8 \mathrm{~Hz}, 1 \mathrm{H}), 6.86-6.80(\mathrm{~m}, 2 \mathrm{H}), 6.79(\mathrm{~d}, J=8.1 \mathrm{~Hz}, 2 \mathrm{H}), 4.69-4.64(\mathrm{~m}, 2 \mathrm{H}), 4.23-4.16(\mathrm{~m}, 2 \mathrm{H}), 4.10$ (q, $J=7.0 \mathrm{~Hz}, 2 \mathrm{H}$ ), 3.84 (s, 3H), 3.80 (dd, $J=9.1,3.4 \mathrm{~Hz}, 2 \mathrm{H}), 3.11-3.05(\mathrm{~m}, 2 \mathrm{H}), 1.37(\mathrm{t}, J=7.0 \mathrm{~Hz}, 3 \mathrm{H})$. ${ }^{13} \mathrm{C}$ NMR $\left(126 \mathrm{MHz}\right.$, Acetone- $\left.d_{6}\right) \delta 148.4,147.5,147.1,146.9,134.2,134.2,119.6,119.6,115.5,115.5$, 111.6, 110.6, 86.7, 86.6, 72.2, 72.2, 65.1, 56.3, 55.3, 49.8, 15.1. HRMS (m/z): $\left[\mathrm{M}-\mathrm{H}_{2} \mathrm{O}+\mathrm{H}\right]^{+}$calcd. for $\mathrm{C}_{21} \mathrm{H}_{23} \mathrm{O}_{5}{ }^{+}, 355.1540$; found, 355.1550.

(+)-29b: ${ }^{1} \mathrm{H}$ NMR (600 MHz, Acetone- $\left.d_{6}\right) \delta 7.46(\mathrm{~s}, 2 \mathrm{H}), 6.97(\mathrm{~d}, J=1.9 \mathrm{~Hz}, 2 \mathrm{H}), 6.83(\mathrm{dd}, J=8.1,1.9 \mathrm{~Hz}$, 2H), $6.78(\mathrm{~d}, J=8.1 \mathrm{~Hz}, 2 \mathrm{H}), 4.66(\mathrm{~d}, J=4.1 \mathrm{~Hz}, 2 \mathrm{H}), 4.22-4.16(\mathrm{~m}, 2 \mathrm{H}), 4.10(\mathrm{q}, J=7.0 \mathrm{~Hz}, 4 \mathrm{H}), 3.79$ $(\mathrm{dd}, J=9.1,3.6 \mathrm{~Hz}, 2 \mathrm{H}), 3.10-3.05(\mathrm{~m}, 2 \mathrm{H}), 1.36(\mathrm{t}, J=7.0 \mathrm{~Hz}, 6 \mathrm{H}) .{ }^{13} \mathrm{C}$ NMR $\left(151 \mathrm{MHz}\right.$, Acetone- $\left.d_{6}\right) \delta$ 
147.6, 147.1, 134.1 119.6, 115.5, 111.6, 86.7, 72.2, 65.1, 55.3, 15.1. HRMS (m/z): $\left[\mathrm{M}-\mathrm{H}_{2} \mathrm{O}+\mathrm{H}\right]^{+}$calcd. for $\mathrm{C}_{22} \mathrm{H}_{25} \mathrm{O}_{5}{ }^{+}, 369.1697$; found, 369.1698. 


\section{Supplementary Figures and Table}

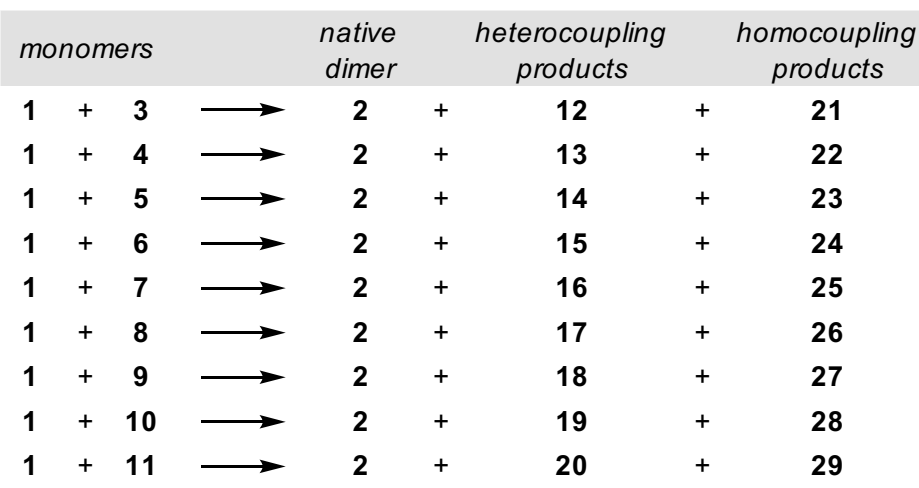

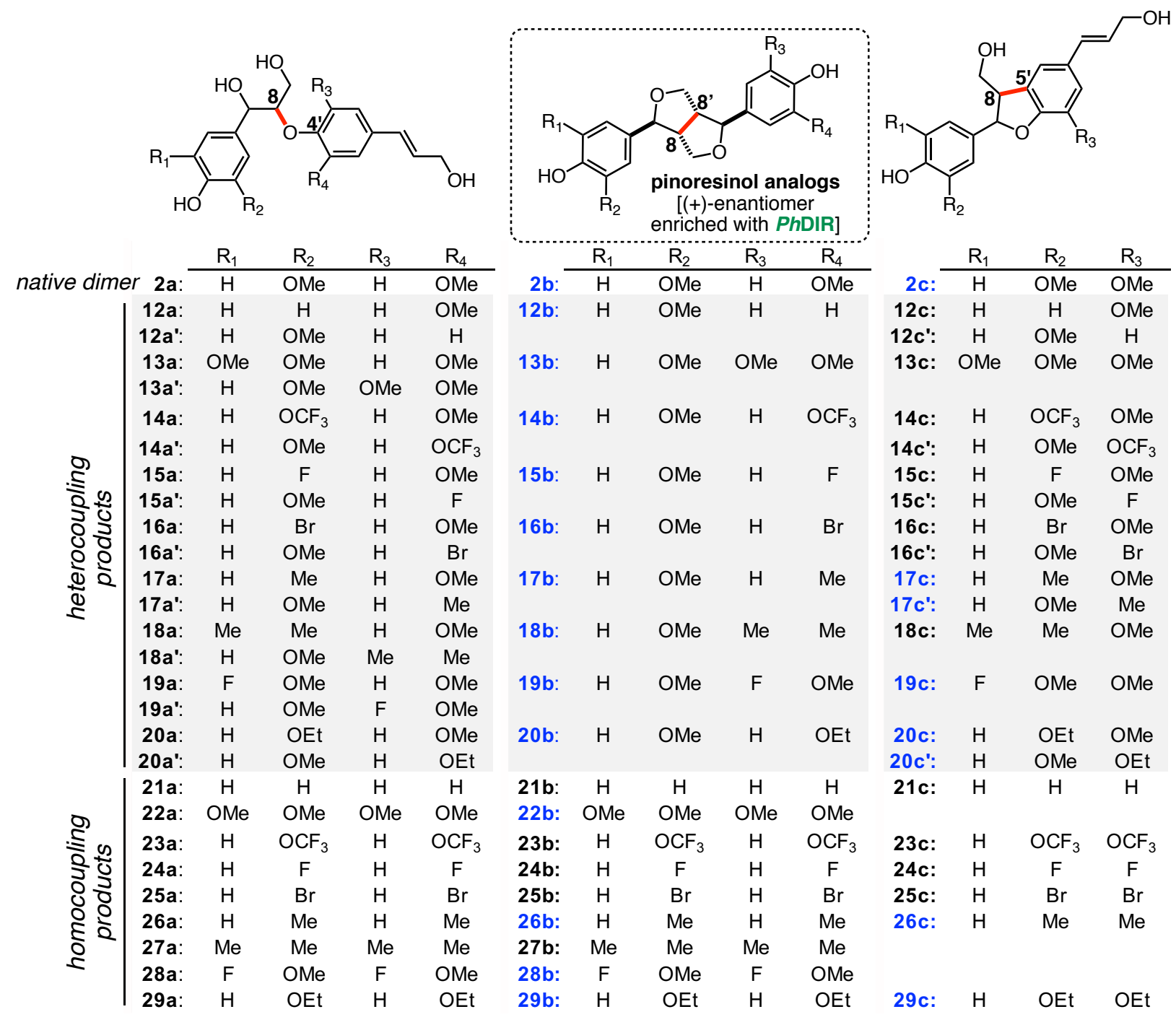

Figure S1. Chemical structure naming convention for all coupling products

Coupling products of the same monomer combination are collectively referred by their assigned compound numbers (table at the top), and specific coupling modes are denoted by lowercase letters: $\mathbf{a}, 8-\mathrm{O}-4^{\prime} ; \mathbf{b}, \mathbf{~}^{-}$ 8'; and c, 8-5' (table at the bottom).

Coupling products that have been structurally characterized in this study are highlighted in blue text. 
All coupling modes

tymn. 8-8' coupling mode
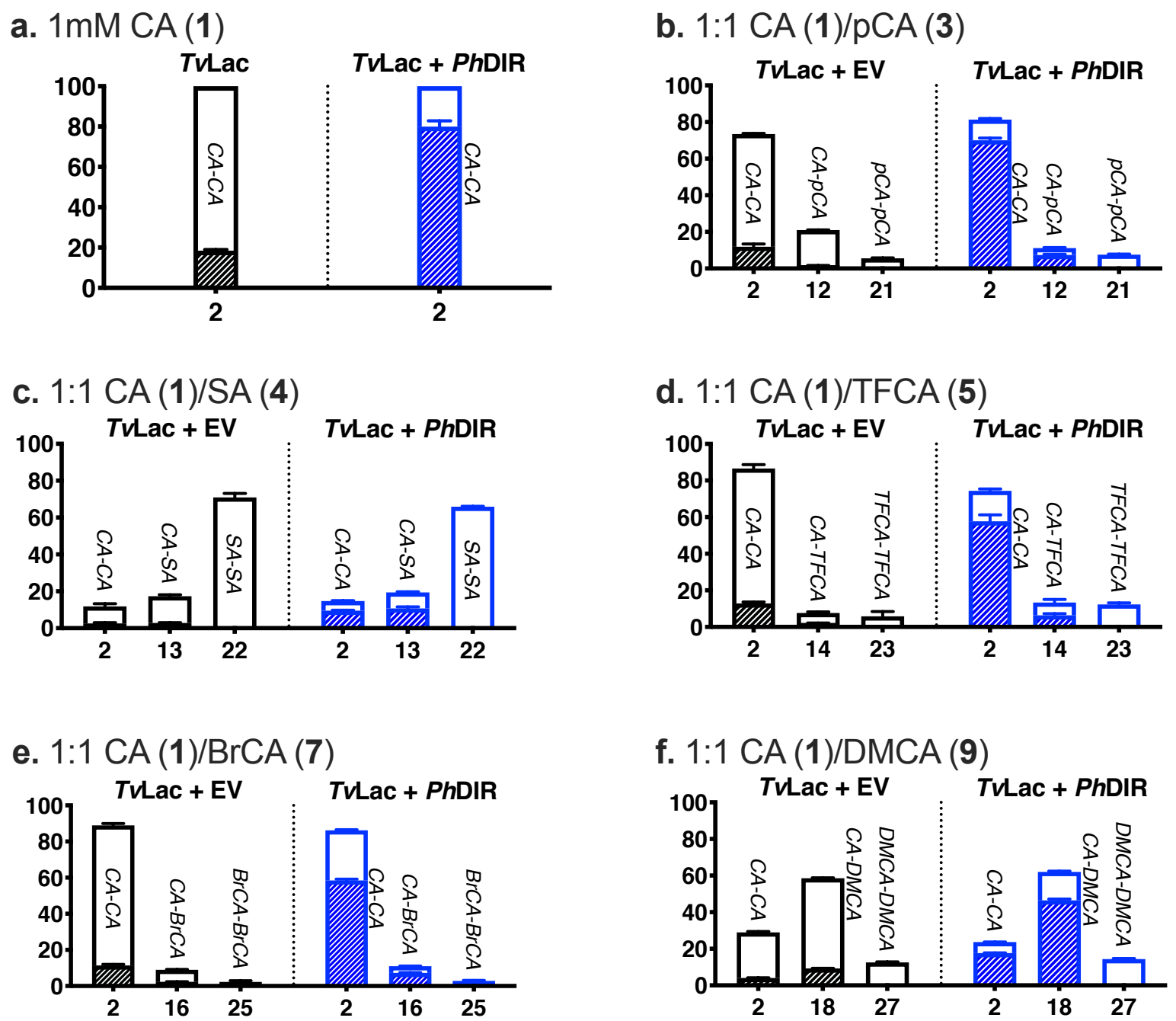

Figure S2. PhDIR mediates regioselective coupling of 1 and heterocoupling of 1 with $3,4,5,7$ and 9 .

Regioselectivity increase is observed in the case of 2 (natural function) but also for 12-18, as indicated by increased heights of filled, slash-pattern bars for 8-8' heterocoupling products. (See Synthetic Procedures for isolation and characterization of racemic 8-8' heterocoupling products from preparatory-scale TVLac reactions.) No regioselectivity increase was observed for homocoupling products of 3-9 (see Figures S3S9).

An equimolar substrate mixture was subjected to TVLac-EV (black) or TVLac-PhDIR (blue) reactions. Yaxis values are normalized the sum of total coupling product content per reaction as calculated by EIC integration of corresponding $\mathrm{m} / \mathrm{z}$ of $\left[\mathrm{M}-\mathrm{H}_{2} \mathrm{O}+\mathrm{H}\right]^{+}$, with $\mathrm{M}$ matching the exact mass of the 8-8' coupling product and its regioisomer, $8-5$ ' coupling product. Bar heights indicate the mean of triplicates, and error bars standard deviations. Full UV chromatograms for equimolar substrate reactions shown in Figures 2 and $\mathbf{S 2}$ are shown in Figure S18. 
a
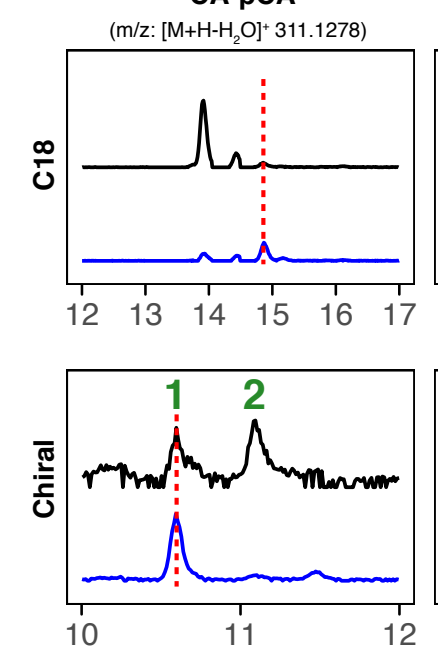

Retention time (min)

b
pCA-pCA

$\left(\mathrm{m} / \mathrm{z}:\left[\mathrm{M}+\mathrm{H}-\mathrm{H}_{2} \mathrm{O}\right]^{+}\right.$281.1172)
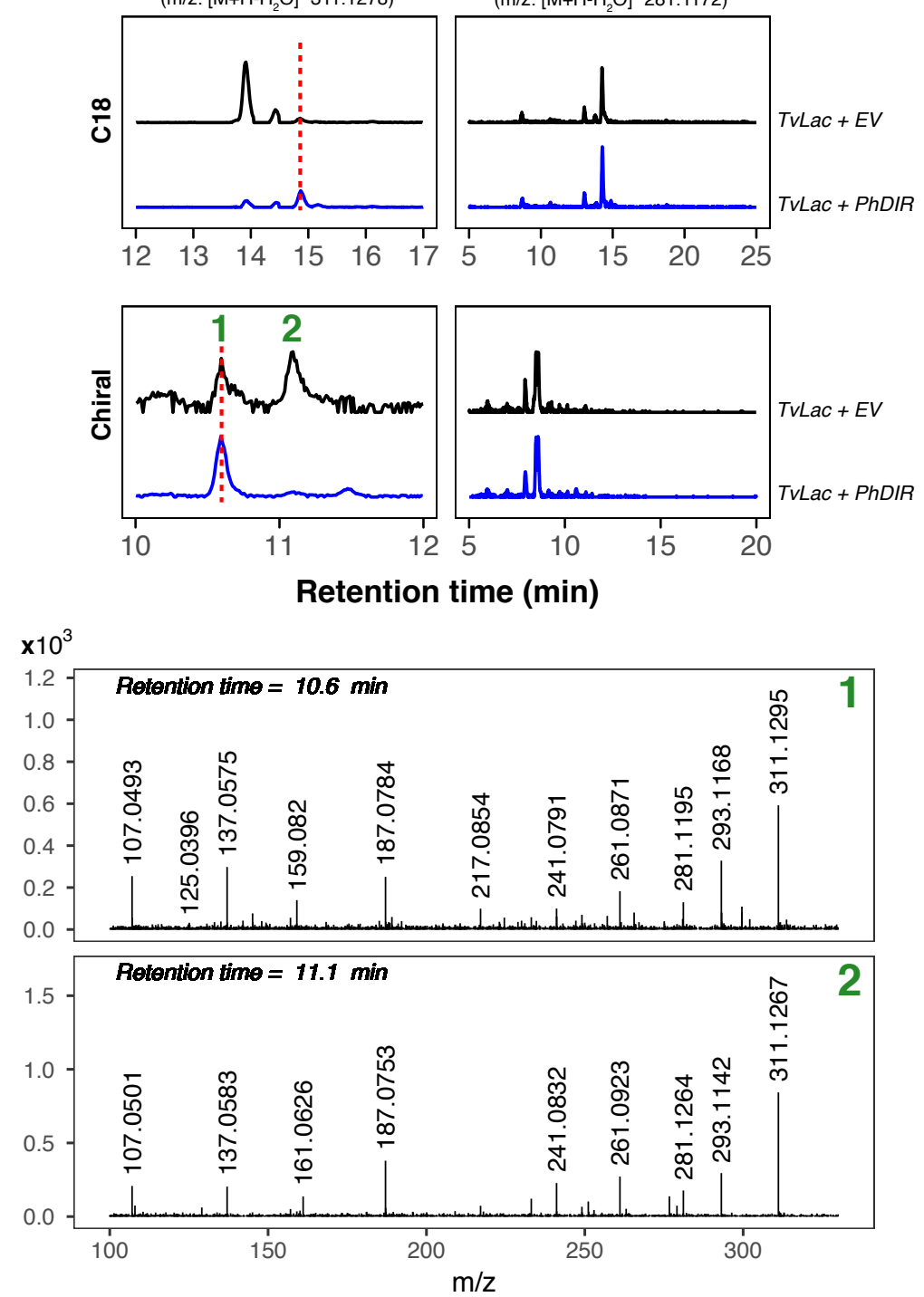

Figure S3. PhDIR directs regio- and stereo-selective heterocoupling of $\mathbf{1}$ and $\mathbf{3}$.

(a) EICs were taken at the corresponding $\mathrm{m} / \mathrm{z}$ values from $\mathrm{C} 18$ (top) and chiral (bottom) chromatographic separations. Black traces are enzymatic products from TVLac reaction supplemented with empty-vector expressing plant extracts, and blue from TVLac and PhDIR extract combination. Chromatographic separation of regioisomers and stereoisomers of heterocoupling products (CA-pCA, 12) is shown on left, and that of homocoupling products (pCA-pCA, 21) on right. In homocoupling, the effect of the presence of $P h D I R$ is indiscernible. The red dotted lines indicate the 8-8' heterocoupling product. C18 traces are to scale to each other, and chiral traces were normalized.

(b) MS/MS fragmentation patterns of the peaks 1 and 2 are indistinguishable, suggesting the two peaks are enantiomers of each other, not regioisomers. Fragmentation voltage of the shown plots is $10 \mathrm{~V}$. 
a
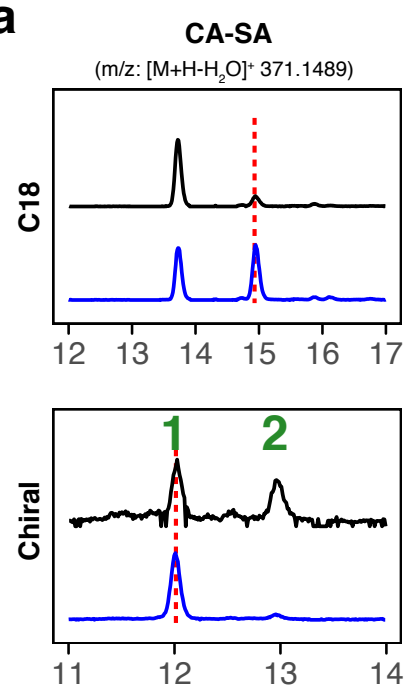

Retention time (min)
SA-SA

$\left(\mathrm{m} / \mathrm{z}:\left[\mathrm{M}+\mathrm{H}-\mathrm{H}_{2} \mathrm{O}\right]^{+} 401.1595\right)$
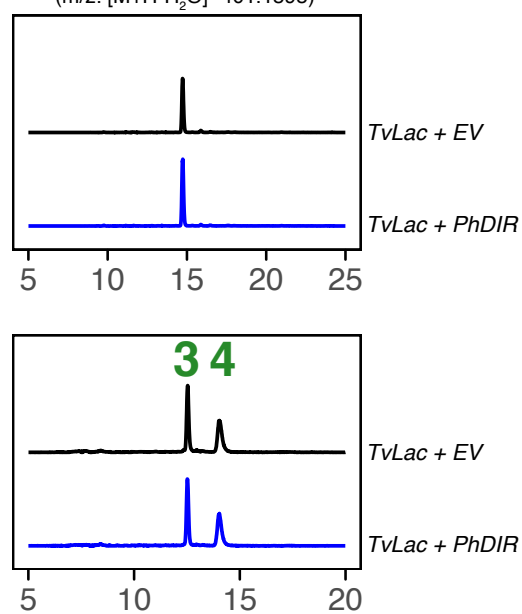

b
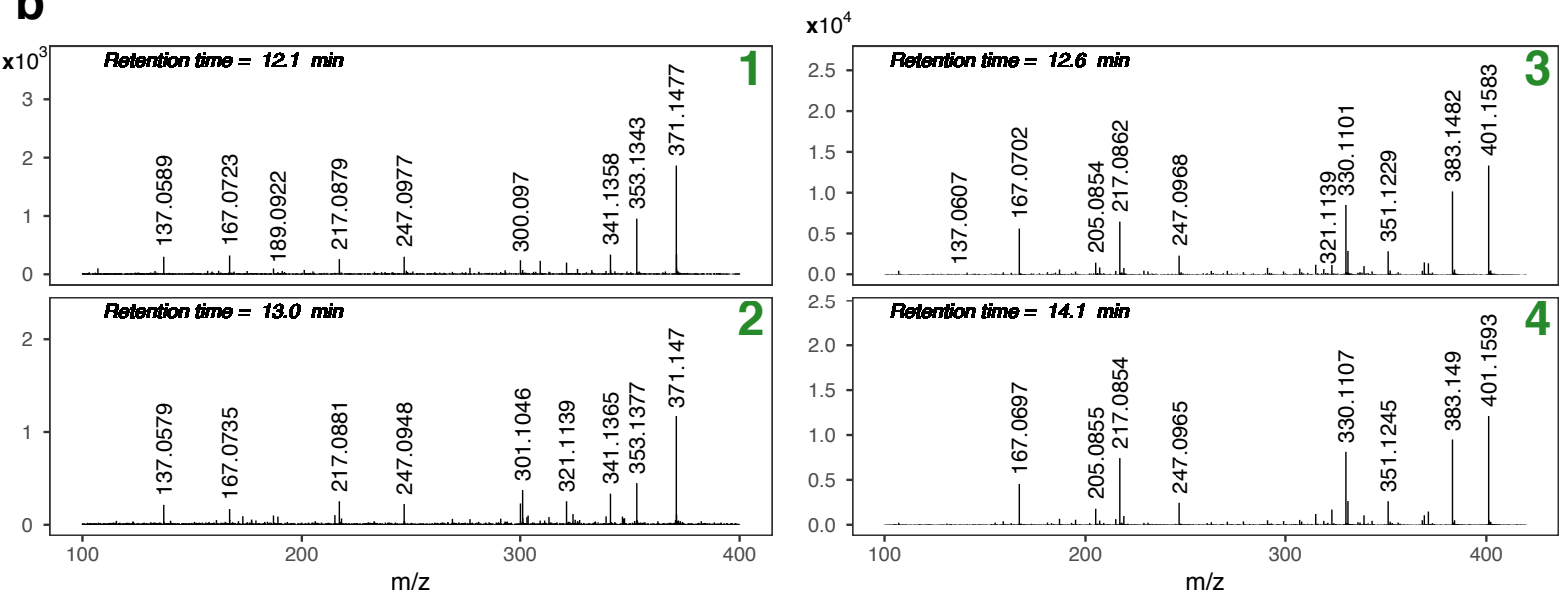

Figure S4. PhDIR directs regio- and stereo-selective heterocoupling of $\mathbf{1}$ and $\mathbf{4}$.

(a) EICs were taken at the corresponding $\mathrm{m} / \mathrm{z}$ values from $\mathrm{C} 18$ (top) and chiral (bottom) chromatographic separations. Black traces are enzymatic products from TVLac reaction supplemented with empty-vector expressing plant extracts, and blue from TVLac and PhDIR extract combination. Chromatographic separation of regioisomers and stereoisomers of heterocoupling products (CA-SA, 13) is shown on left, and that of homocoupling products (SA-SA, 22) on right. In homocoupling, the effect of the presence of PhDIR is indiscernible. Notably, the major homocoupling product shown is likely the 8-8' coupling product, as 8-5' homocoupling product of 4 cannot be formed due to the methoxy functional group at the 5' position of the monomer. However, no dirigent protein activity is observed; the enantiomeric separation profile of the homocoupling product is unchanged with the presence of PhDIR. The red dotted lines indicate the 8-8' heterocoupling product. C18 traces are to scale to each other, and chiral traces were normalized.

(b) MS/MS fragmentation patterns of the peaks 1 and 2 are indistinguishable, so are the peaks 3 and 4 , suggesting each of the pairs of the two peaks are enantiomers of each other. Peaks 1 and 2 are heterocoupled enantiomers, and 3 and 4 homocoupled enantiomers. Fragmentation voltage of the shown plots is $10 \mathrm{~V}$. 
a
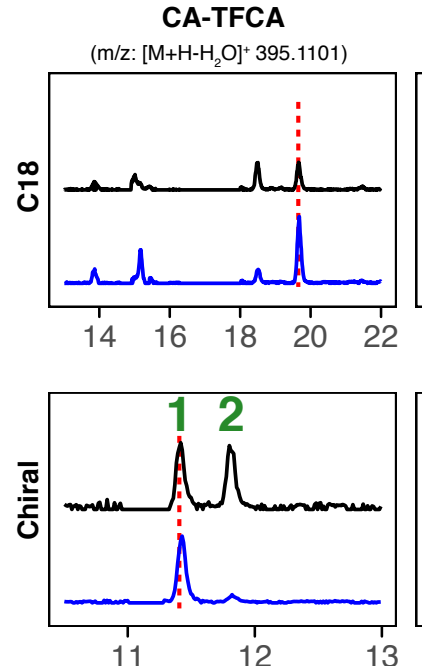

Retention time (min)

b
TFCA-TFCA

$\left(\mathrm{m} / \mathrm{z}:\left[\mathrm{M}+\mathrm{H}-\mathrm{H}_{2} \mathrm{O}\right]^{+} 449.0818\right)$
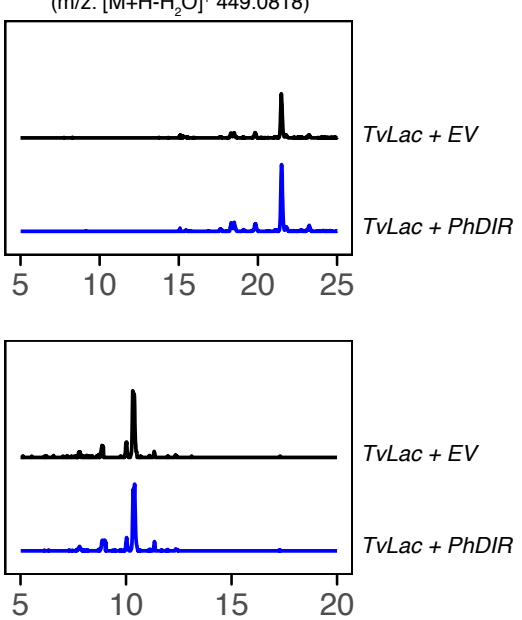

$\times 10^{3}$
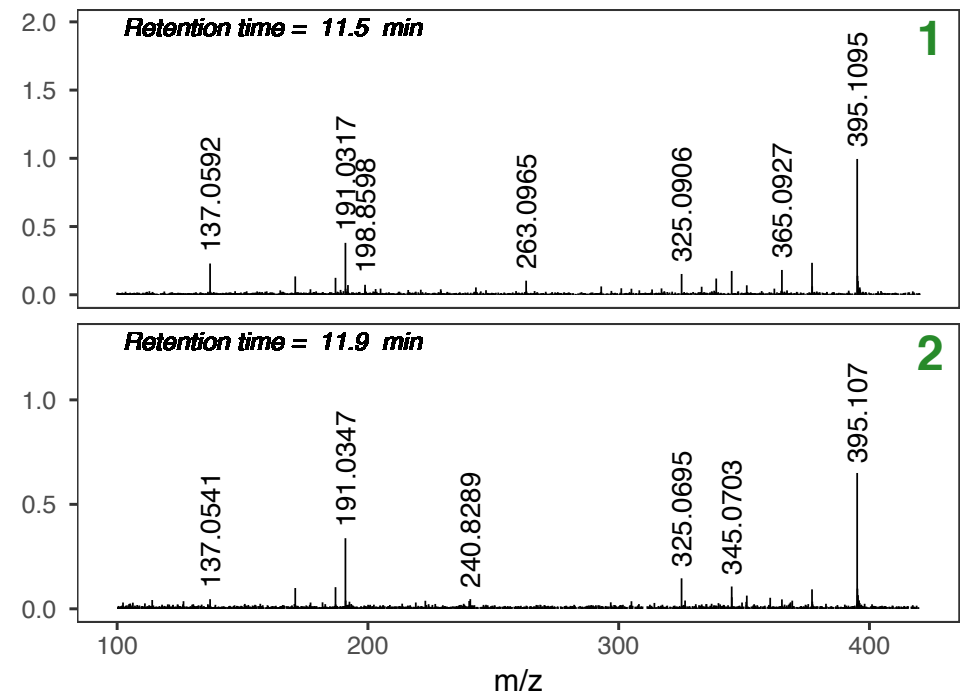

Figure S5. PhDIR directs regio- and stereo-selective heterocoupling of $\mathbf{1}$ and $\mathbf{5}$.

(a) EICs were taken at the corresponding $\mathrm{m} / \mathrm{z}$ values from $\mathrm{C} 18$ (top) and chiral (bottom) chromatographic separations. Black traces are enzymatic products from TVLac reaction supplemented with empty-vector expressing plant extracts, and blue from TVLac and PhDIR extract combination. Chromatographic separation of regioisomers and stereoisomers of heterocoupling products (CA-TFCA, 14) is shown on left, and that of homocoupling products (TFCA-TFCA, 23) on right. In homocoupling, the effect of the presence of $P h D I R$ is indiscernible. The red dotted lines indicate the 8-8' heterocoupling product. C18 traces are to scale to each other, and chiral traces were normalized.

(b) MS/MS fragmentation patterns of the peaks 1 and 2 are indistinguishable from each other, suggesting the two peaks are enantiomers of each other. Fragmentation voltage of the shown plots is $10 \mathrm{~V}$. 
a
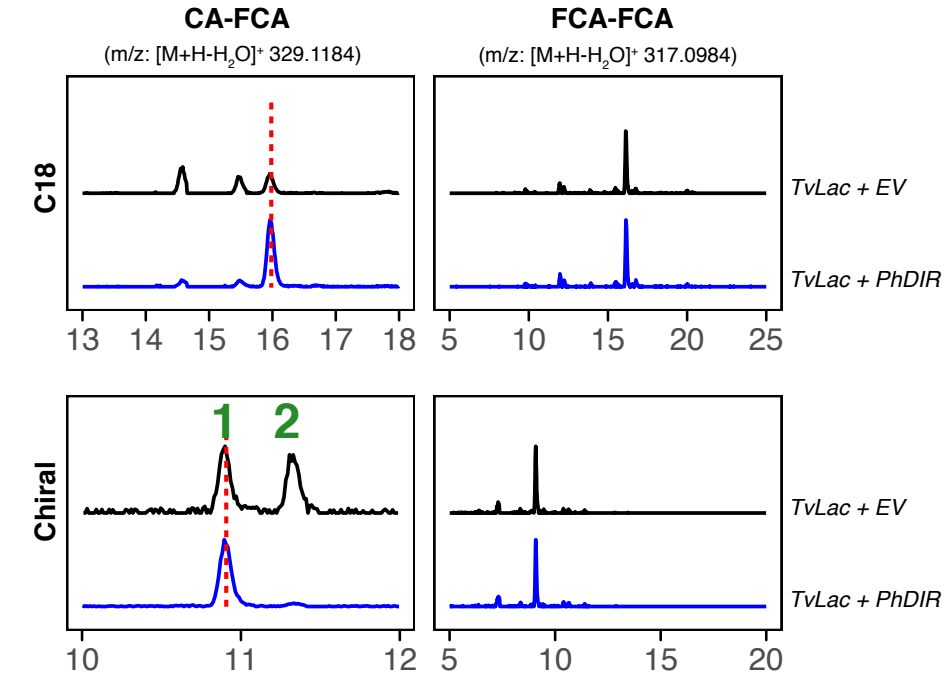

b

Retention time ( $\mathrm{min})$

$\mathbf{x} 10^{3}$
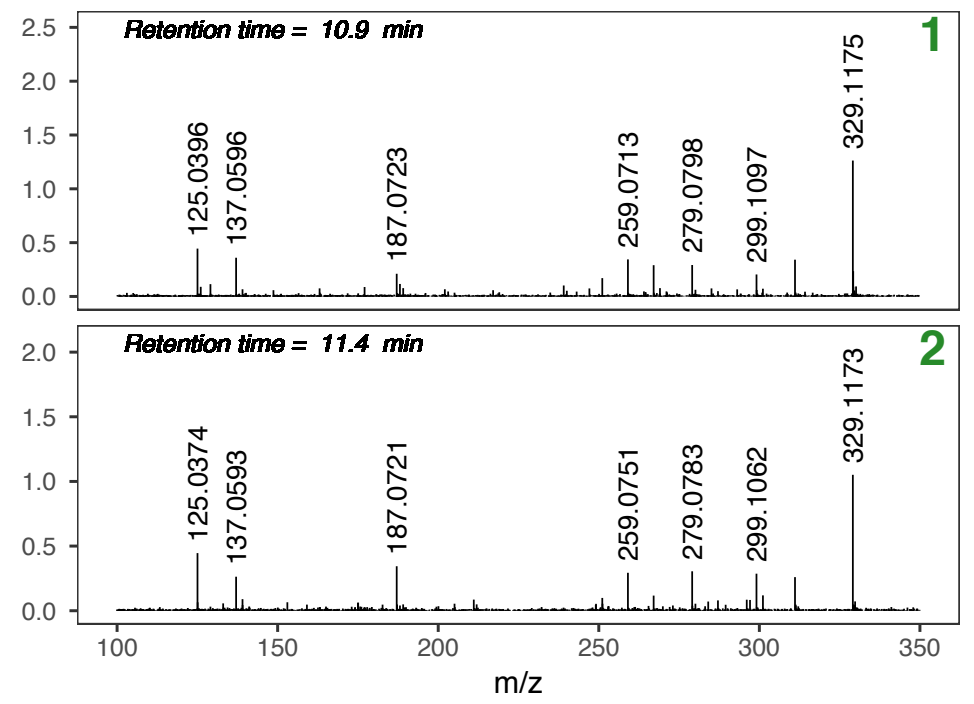

Figure S6. PhDIR directs regio- and stereo-selective heterocoupling of $\mathbf{1}$ and $\mathbf{6}$.

(a) EICs were taken at the corresponding $\mathrm{m} / \mathrm{z}$ values from $\mathrm{C} 18$ (top) and chiral (bottom) chromatographic separations. Black traces are enzymatic products from TVLac reaction supplemented with empty-vector expressing plant extracts, and blue from TVLac and PhDIR extract combination. Chromatographic separation of regioisomers and stereoisomers of heterocoupling products (CA-FCA, 15) is shown on left, and that of homocoupling products (FCA-FCA, 24) on right. In homocoupling, the effect of the presence of PhDIR is indiscernible. The red dotted line indicates the 8-8' heterocoupling product. C18 traces are to scale to each other, and chiral traces were normalized.

(b) MS/MS fragmentation patterns of the peaks 1 and 2 are indistinguishable from each other, suggesting the two peaks are enantiomers of each other. Fragmentation voltage of the shown plots is $10 \mathrm{~V}$. 
a
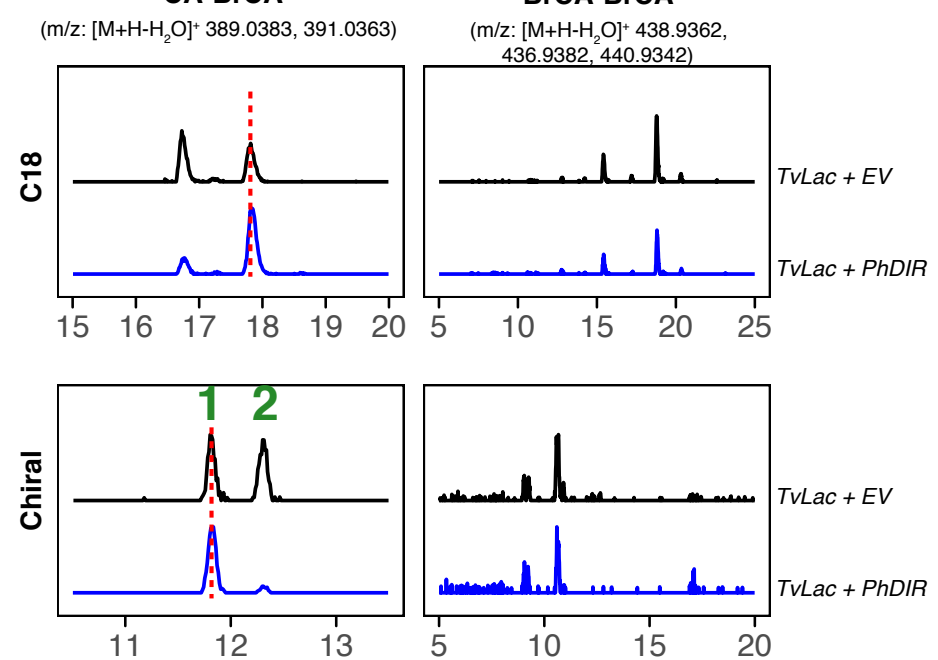

b
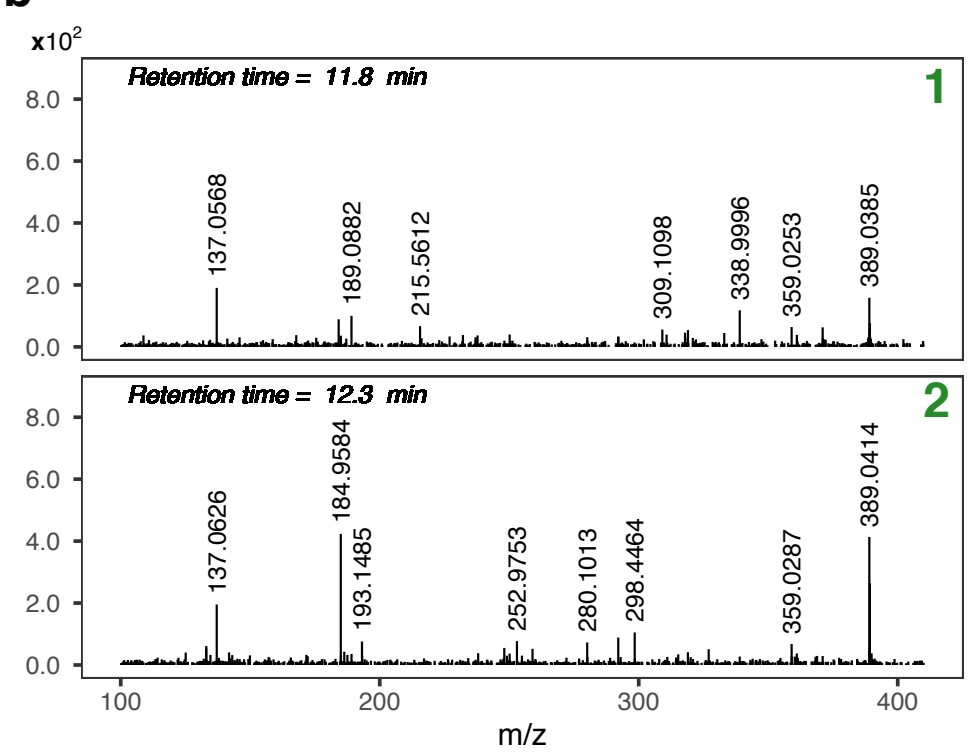

Figure S7. PhDIR directs regio- and stereo-selective heterocoupling of $\mathbf{1}$ and $\mathbf{7 .}$

(a) EICs were taken at the corresponding $\mathrm{m} / \mathrm{z}$ values from $\mathrm{C} 18$ (top) and chiral (bottom) chromatographic separations. Black traces are enzymatic products from TVLac reaction supplemented with empty-vector expressing plant extracts, and blue from TVLac and PhDIR extract combination. Chromatographic separation of regioisomers and stereoisomers of heterocoupling products (CA-BrCA, 16) is shown on left, and that of homocoupling products (BrCA-BrCA, 25) on right. In homocoupling, the effect of the presence of $P h D I R$ is indiscernible. The red dotted lines indicate the 8-8' heterocoupling product. $\mathrm{C} 18$ traces are to scale to each other, and chiral traces were normalized.

(b) MS/MS fragmentation patterns of the peaks 1 and 2 are indistinguishable from each other, suggesting the two peaks are enantiomers of each other. Fragmentation voltage of the shown plots is $10 \mathrm{~V}$. 


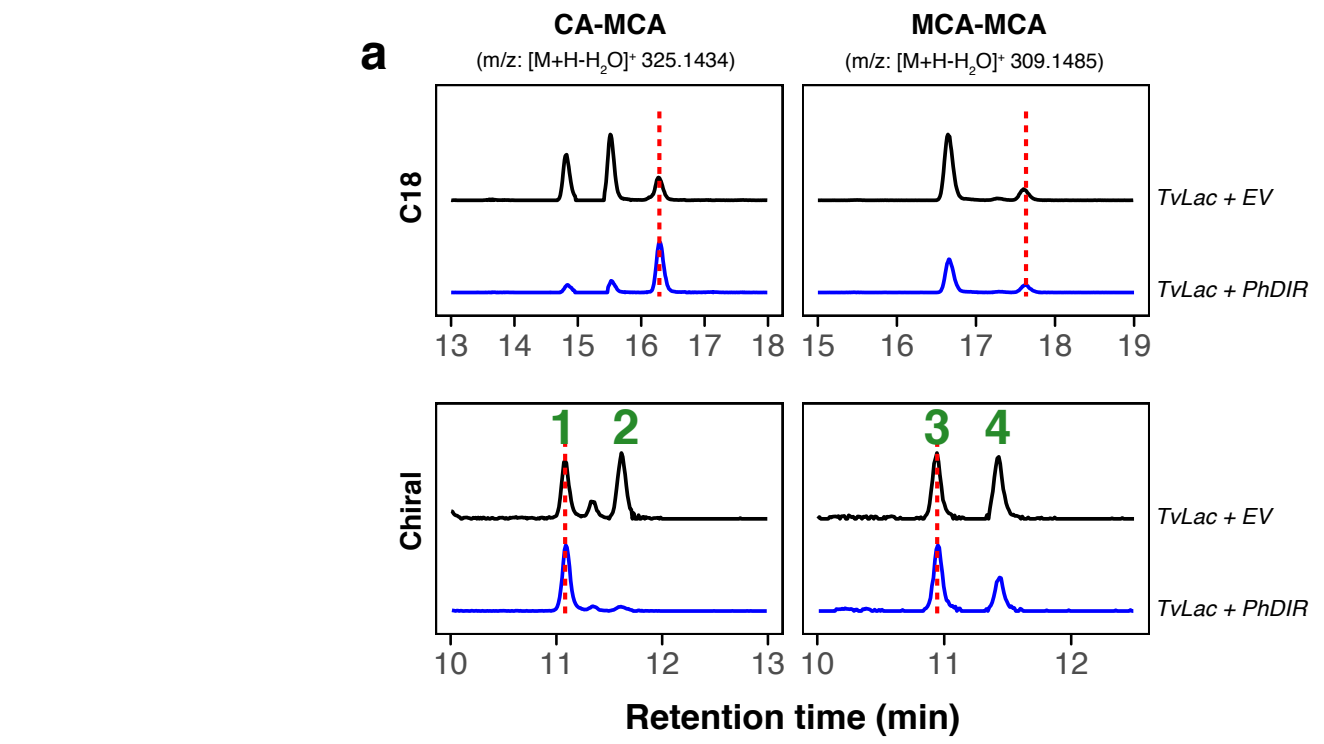

MCA-MCA

b
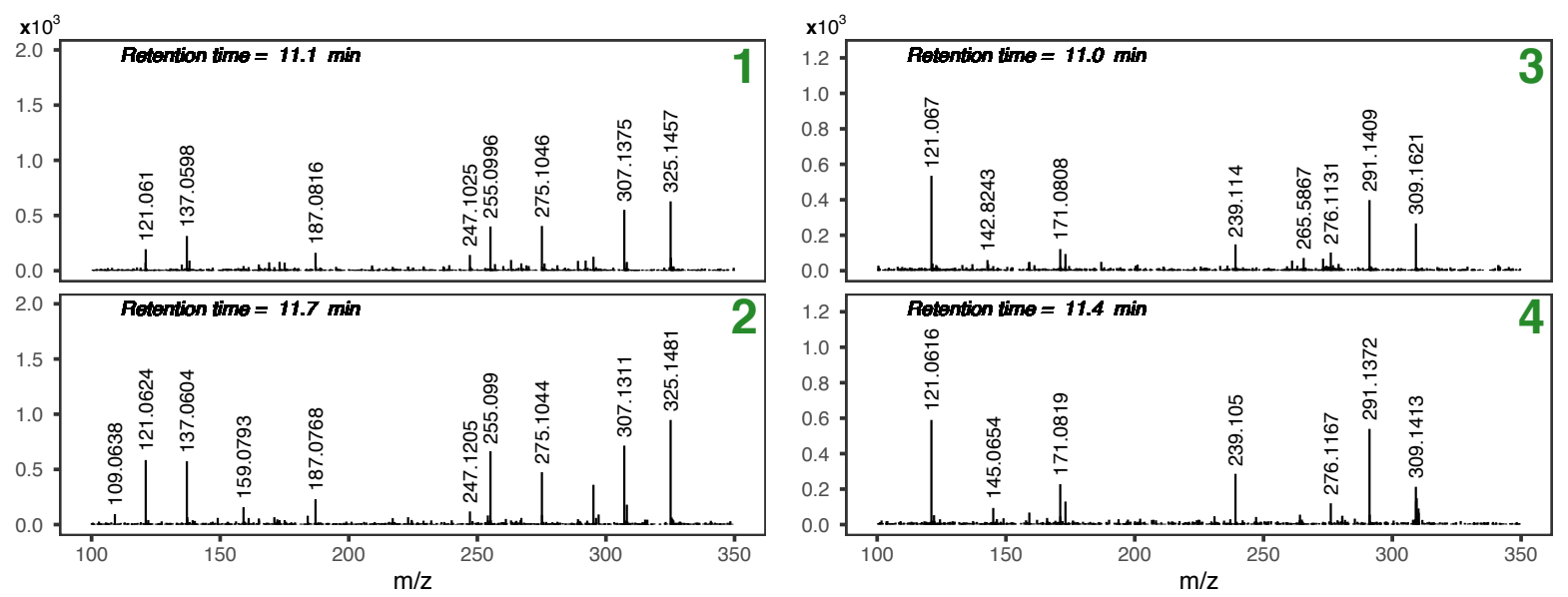

Figure S8. PhDIR directs regio- and stereo-selective heterocoupling of $\mathbf{1}$ and $\mathbf{8}$.

(a) EICs were taken at the corresponding $\mathrm{m} / \mathrm{z}$ values from $\mathrm{C} 18$ (top) and chiral (bottom) chromatographic separations. Black traces are enzymatic products from TVLac reaction supplemented with empty-vector expressing plant extracts, and blue from TVLac and PhDIR extract combination. Chromatographic separation of regioisomers and stereoisomers of heterocoupling products (CA-MCA, 17) is shown on left, and that of homocoupling products (MCA-MCA, 26) on right. In homocoupling, the effect of the presence of $P h D I R$ is indiscernible. The red dotted lines indicate the 8-8' heterocoupling product. C18 traces are to scale to each other, and chiral traces were normalized.

(b) MS/MS fragmentation patterns of the peaks 1 and 2 are indistinguishable, so are the peaks 3 and 4 , suggesting each of the pairs of the two peaks are enantiomers of each other. Peaks 1 and 2 are heterocoupled enantiomers, and 3 and 4 homocoupled enantiomers. Fragmentation voltage of the shown plots is $10 \mathrm{~V}$. 
a
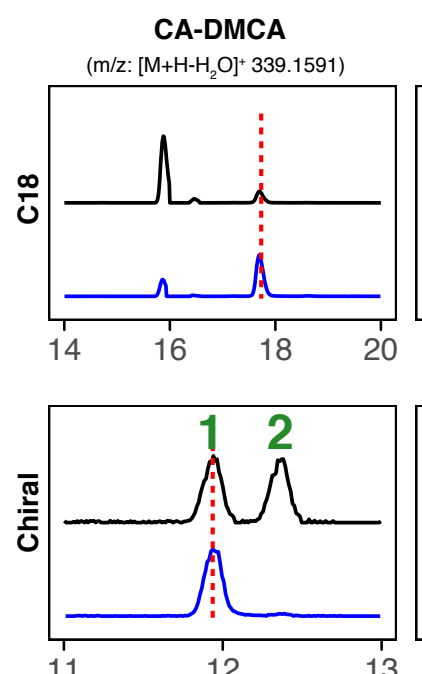

11

b

DMCA-DMCA

$\left(\mathrm{m} / \mathrm{z}:\left[\mathrm{M}+\mathrm{H}-\mathrm{H}_{2} \mathrm{O}\right]^{+} 337.1798\right)$
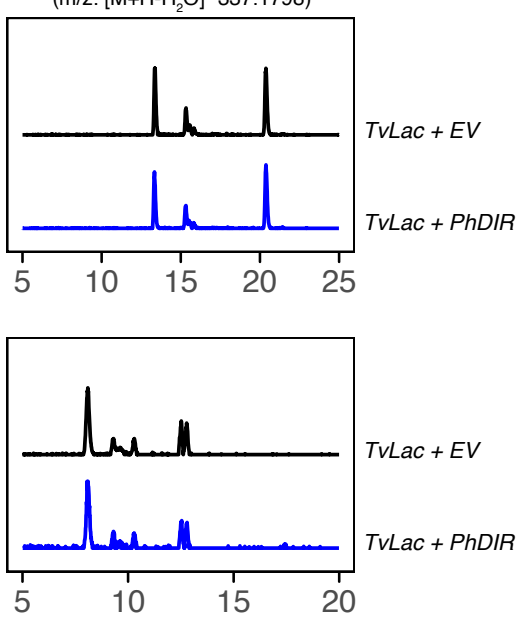

Retention time (min)

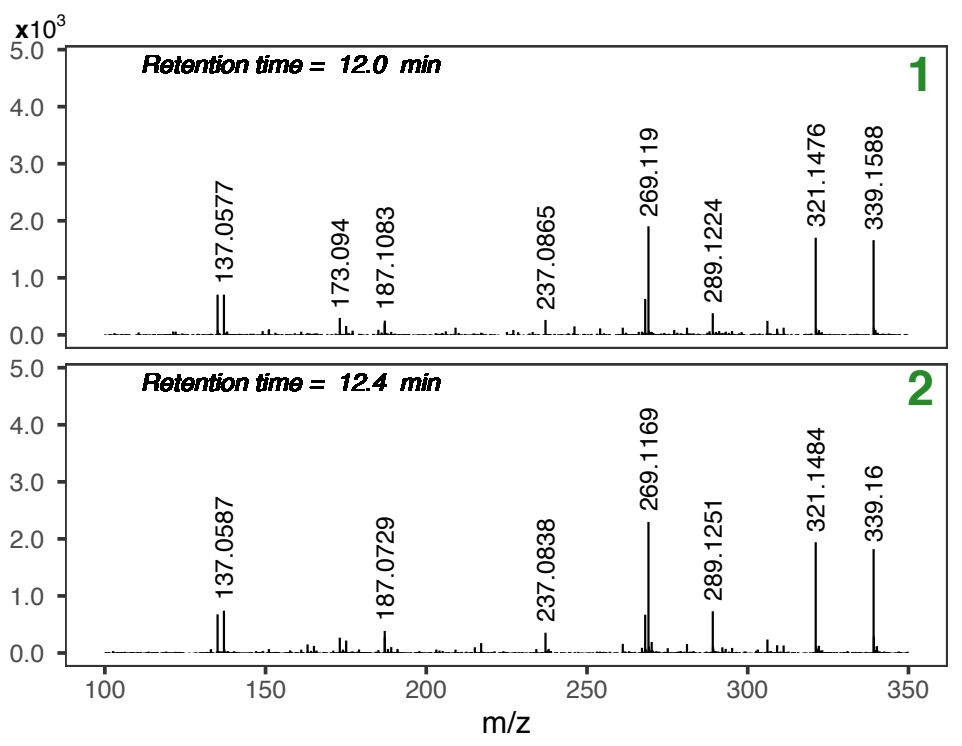

Figure S9. PhDIR directs regio- and stereo-selective heterocoupling of $\mathbf{1}$ and $\mathbf{9}$.

(a) EICs were taken at the corresponding $\mathrm{m} / \mathrm{z}$ values from $\mathrm{C} 18$ (top) and chiral (bottom) chromatographic separations. Black traces are enzymatic products from TVLac reaction supplemented with empty-vector expressing plant extracts, and blue from TULac and PhDIR extract combination. Chromatographic separation of regioisomers and stereoisomers of heterocoupling products (CA-DMCA, 18) is shown on left, and that of homocoupling products (DMCA-DMCA, 27) on right. In homocoupling, the effect of the presence of $P h D I R$ is indiscernible. The red dotted lines indicate the 8-8' heterocoupling product. $\mathrm{C} 18$ traces are to scale to each other, and chiral traces were normalized.

(b) MS/MS fragmentation patterns of the peaks 1 and 2 are indistinguishable from each other, suggesting the two peaks are enantiomers of each other. Fragmentation voltage of the shown plots is $10 \mathrm{~V}$. 

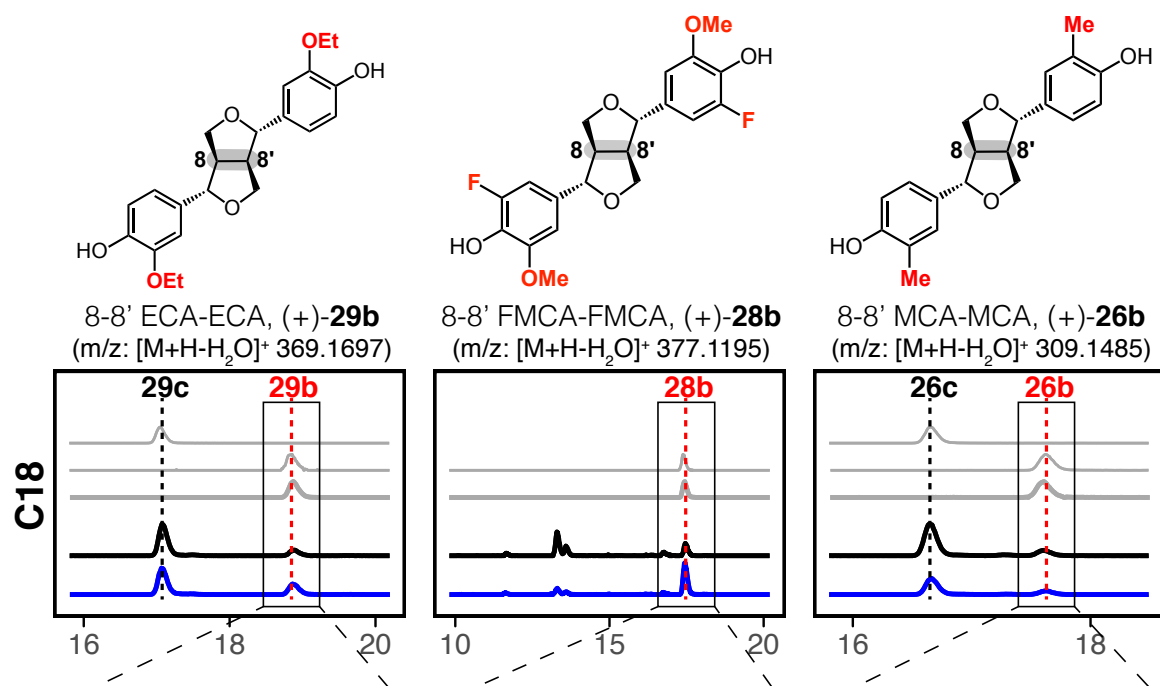

8-8' FMCA-FMCA, (+)-28b $\left(\mathrm{m} / \mathrm{z}:\left[\mathrm{M}+\mathrm{H}-\mathrm{H}_{2} \mathrm{O}\right]^{+} 377.1195\right)$

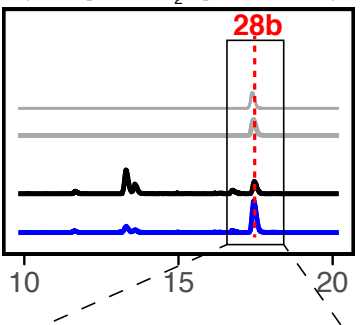

8-8' MCA-MCA (+)-26b $\left(\mathrm{m} / \mathrm{z}:\left[\mathrm{M}+\mathrm{H}-\mathrm{H}_{2} \mathrm{O}\right]^{+} 309.1485\right)$
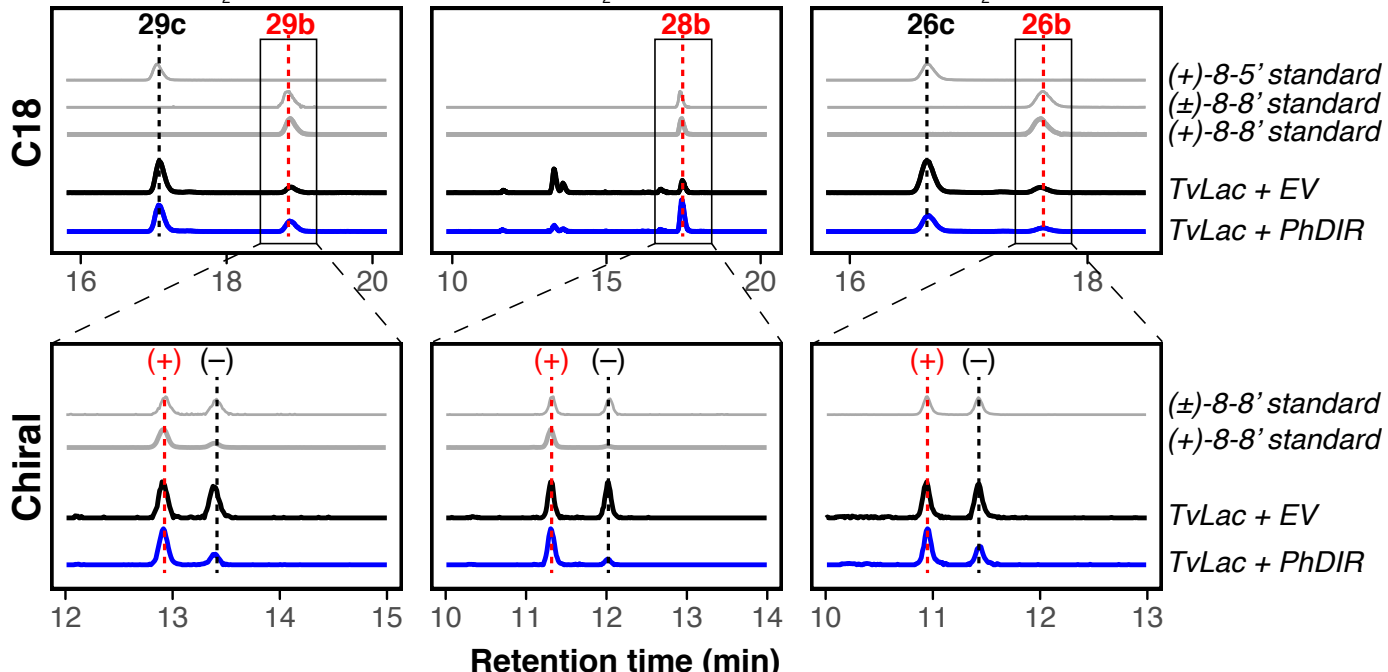

Figure S10. PhDIR mediates regio- and stereoselective homocoupling of $\mathbf{8 , 1 0}$, and $\mathbf{1 1 .}$

EICs at the corresponding $\mathrm{m} / \mathrm{z}$ values of the homocoupling products show regioisomeric separation on the C18 column (top) and stereoisomer separation on the chiral column (bottom). Enantiomeric and racemic standards (grey traces) were isolated from preparatory scale reactions. (See Synthetic Procedures) Black traces are enzymatic products of TVLac oxidation supplemented with empty-vector expressing plant extracts, and blue traces from TVLac and PhDIR extract combination reaction. Regio- and enantioselective product of the PhDIR-mediated coupling reaction is indicated by the red dotted lines. Chromatographic traces of enzymatic reactions on $\mathrm{C} 18$ are to scale to each other, and those on chiral column and standard peaks (shown in grey) are normalized. 
a. Enantiomers of hetero-ethoxy-pinoresinol (20b)

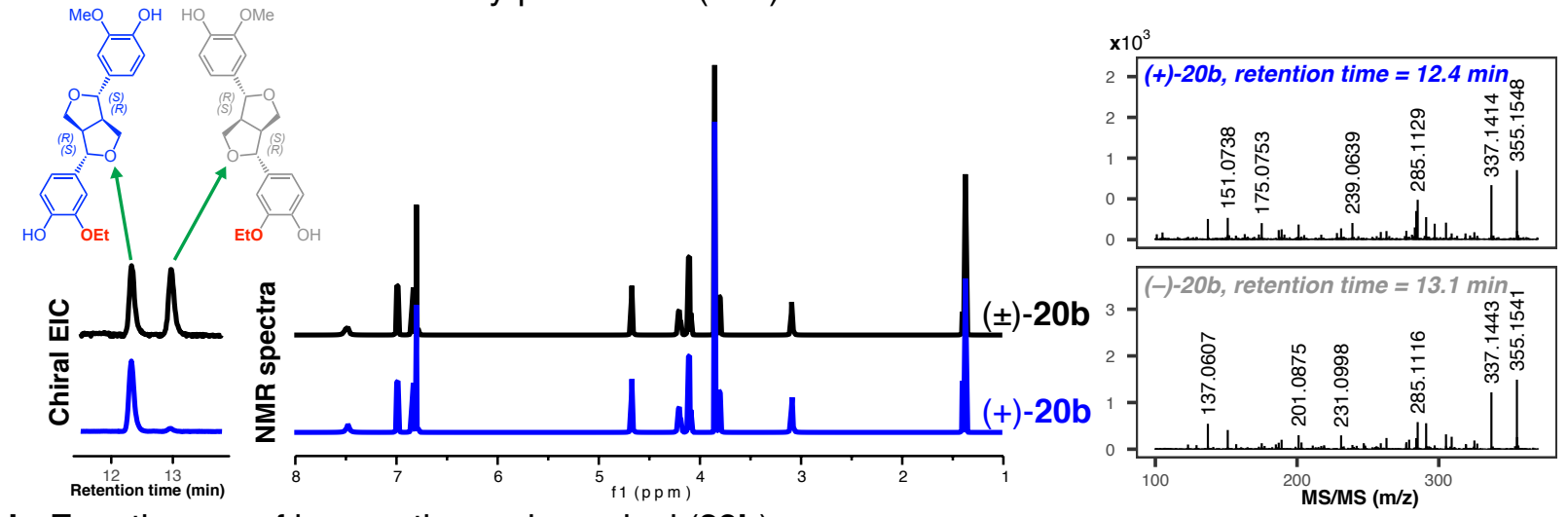

b. Enantiomers of homo-ethoxy-pinoresinol (29b)
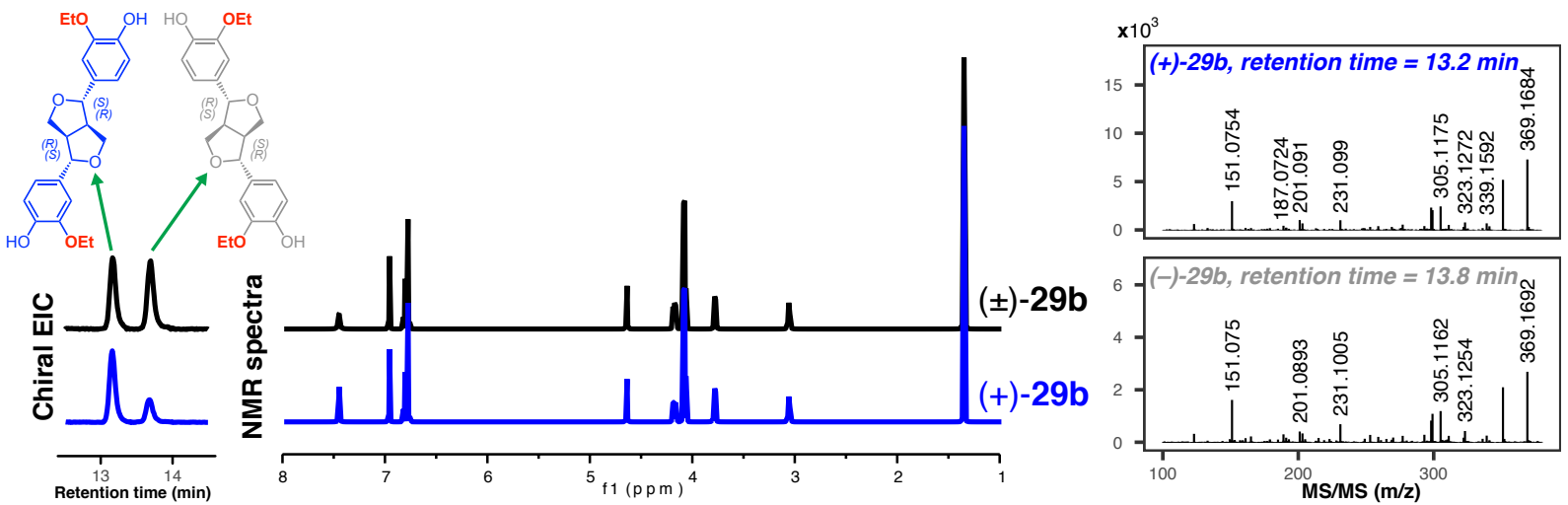

c. Enantiomers of hetero-fluoro-pinoresinol (19b)
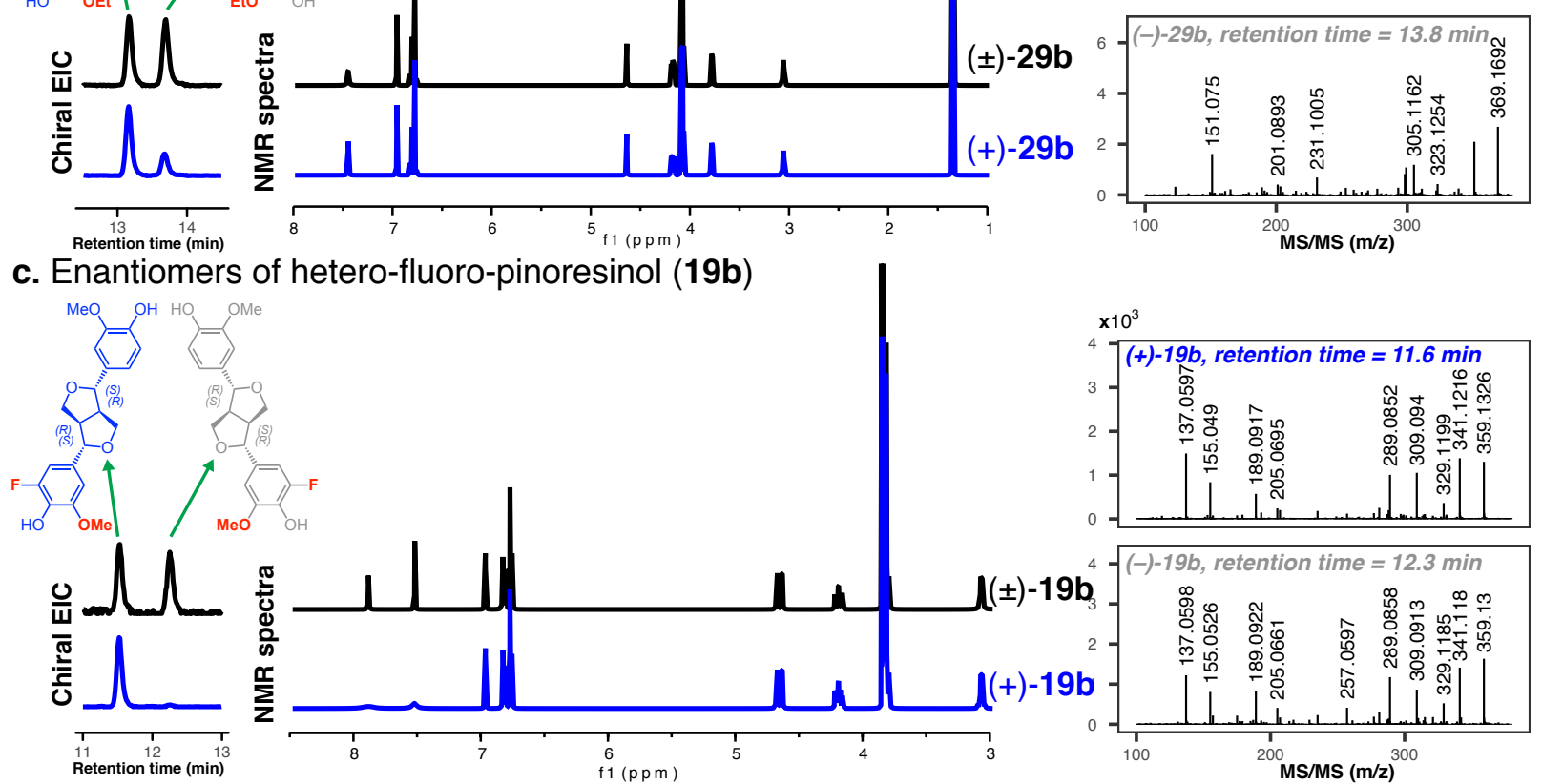

c. Enantiomers

d. Enantiomers of homo-fluoro-pinoresinol (28b)
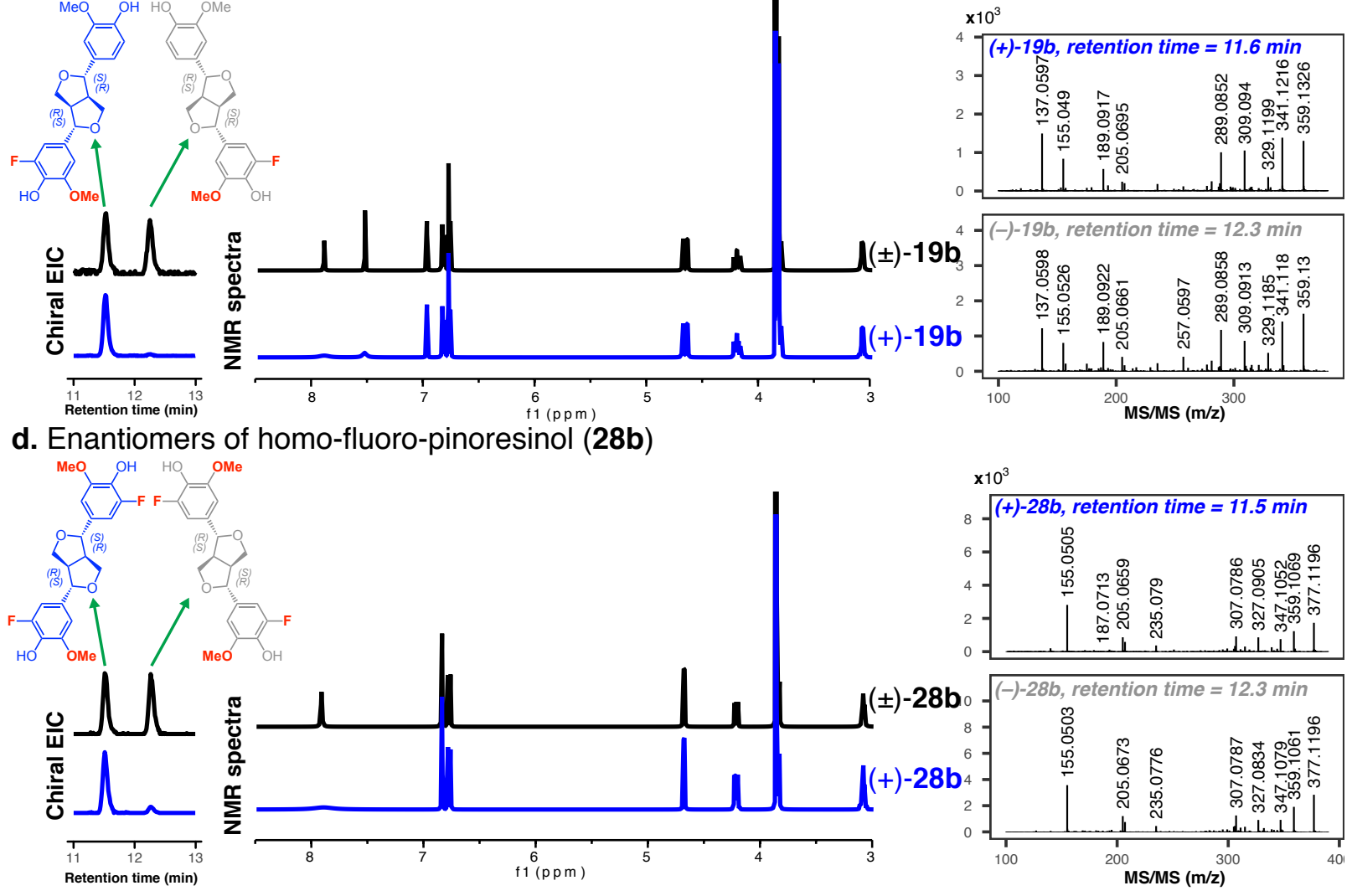

$\left.\right|_{3} ^{( \pm)-28 b}$
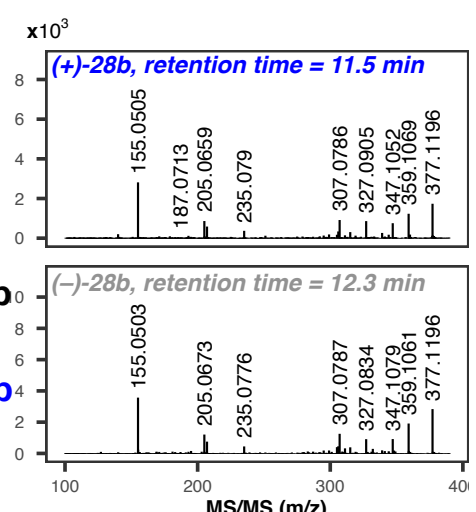
Figure S11. Two peaks separated by chiral chromatography are racemic.

EICs at $\mathrm{m} / \mathrm{z}$ of $\left[\mathrm{M}+\mathrm{H}_{2} \mathrm{O}-\mathrm{H}\right]^{+}$and NMR spectra of racemic (black) and enantiomeric (blue) samples were analyzed, and MS/MS fragmentations of the doublet peaks separated on the chiral column as indicated by green arrows pointing to the corresponding chemical structures were compared for (a) $20 \mathrm{~b}(\mathrm{~m} / \mathrm{z}=$ 355.1540), (b) $29 b(\mathrm{~m} / \mathrm{z}=369.1697)$, (c) $19 b(\mathrm{~m} / \mathrm{z}=359.1389)$, and (d) $28 b(\mathrm{~m} / \mathrm{z}=377.1195)$. Racemic samples were purified from preparatory-scale TVLac reactions, and enantiomeric samples from preparatory-scale TVLac-PhDIR reactions as described in Synthetic Procedures. In the NMR spectra shown here, solvent and impurity peaks are not shown; see the NMR Spectra section for raw spectral data. Equally sized peaks in the racemic sample observed on chiral chromatography suggests enantiomeric separation. The NMR spectra of racemic and enantio-enriched samples appear equivalent, as well as MS/MS fragmentation patterns of the two peaks separated by chiral chromatography, as both MS/MS fragmentation and NMR taken without a chiral shift reagent do not distinguish enantiomers. 
a. Homocoupling products of 1

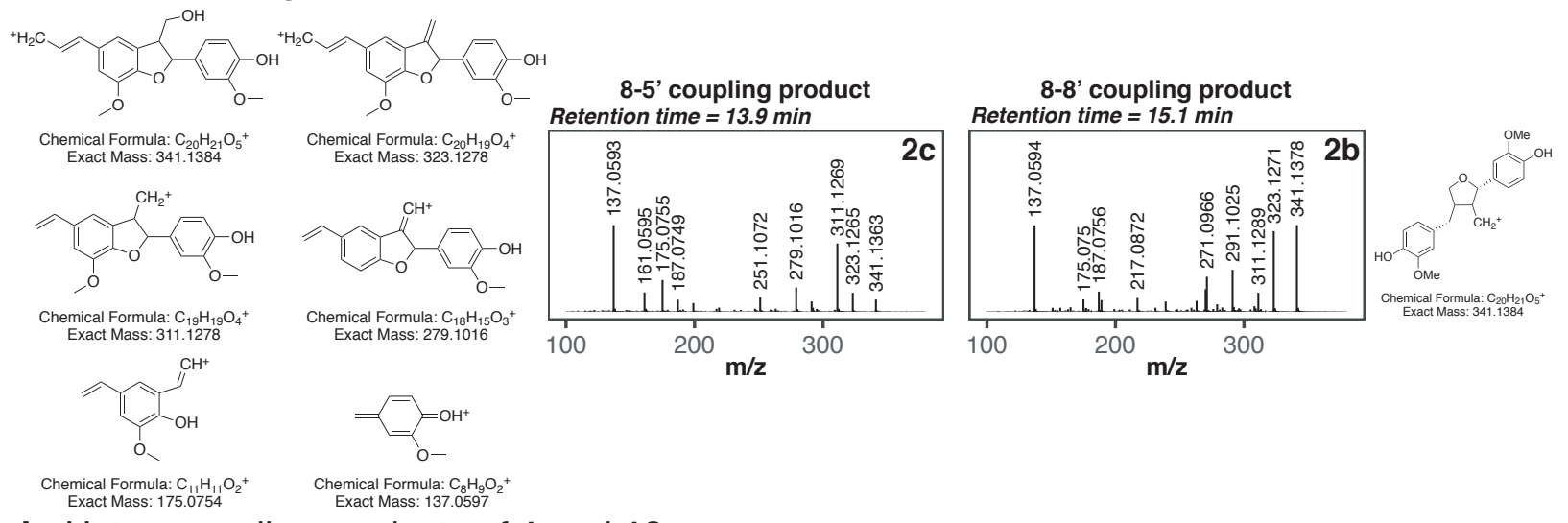

b. Heterocoupling products of $\mathbf{1}$ and $\mathbf{1 0}$

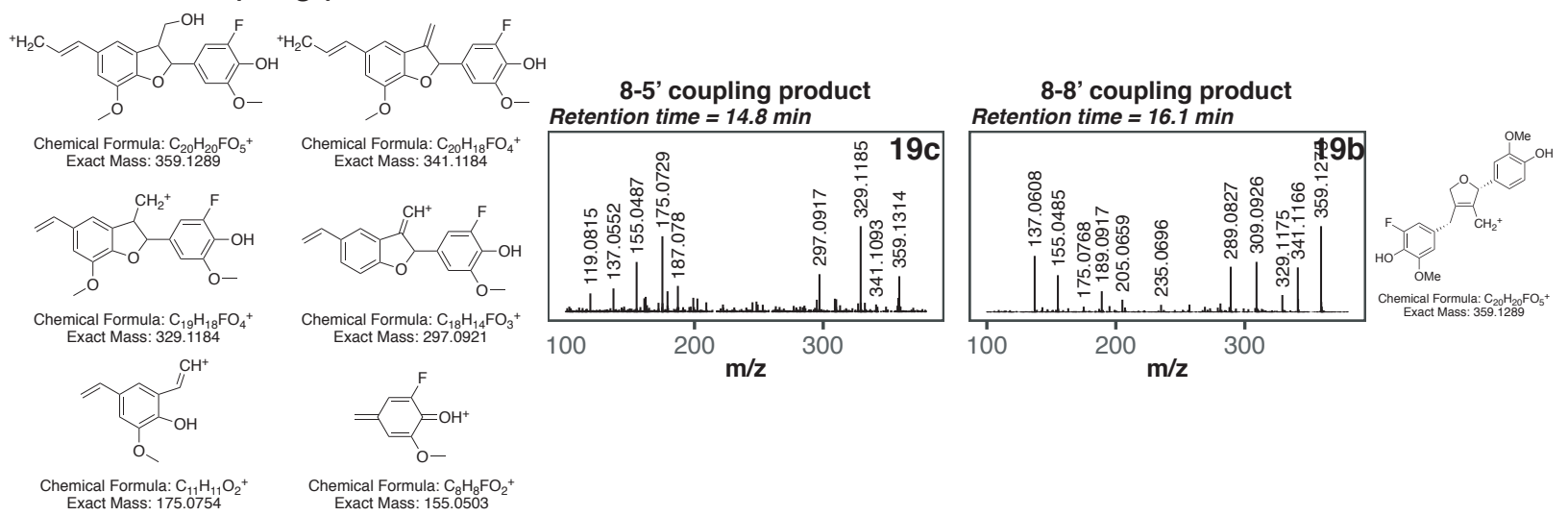

c. Heterocoupling products of $\mathbf{1}$ and $\mathbf{1 1}$

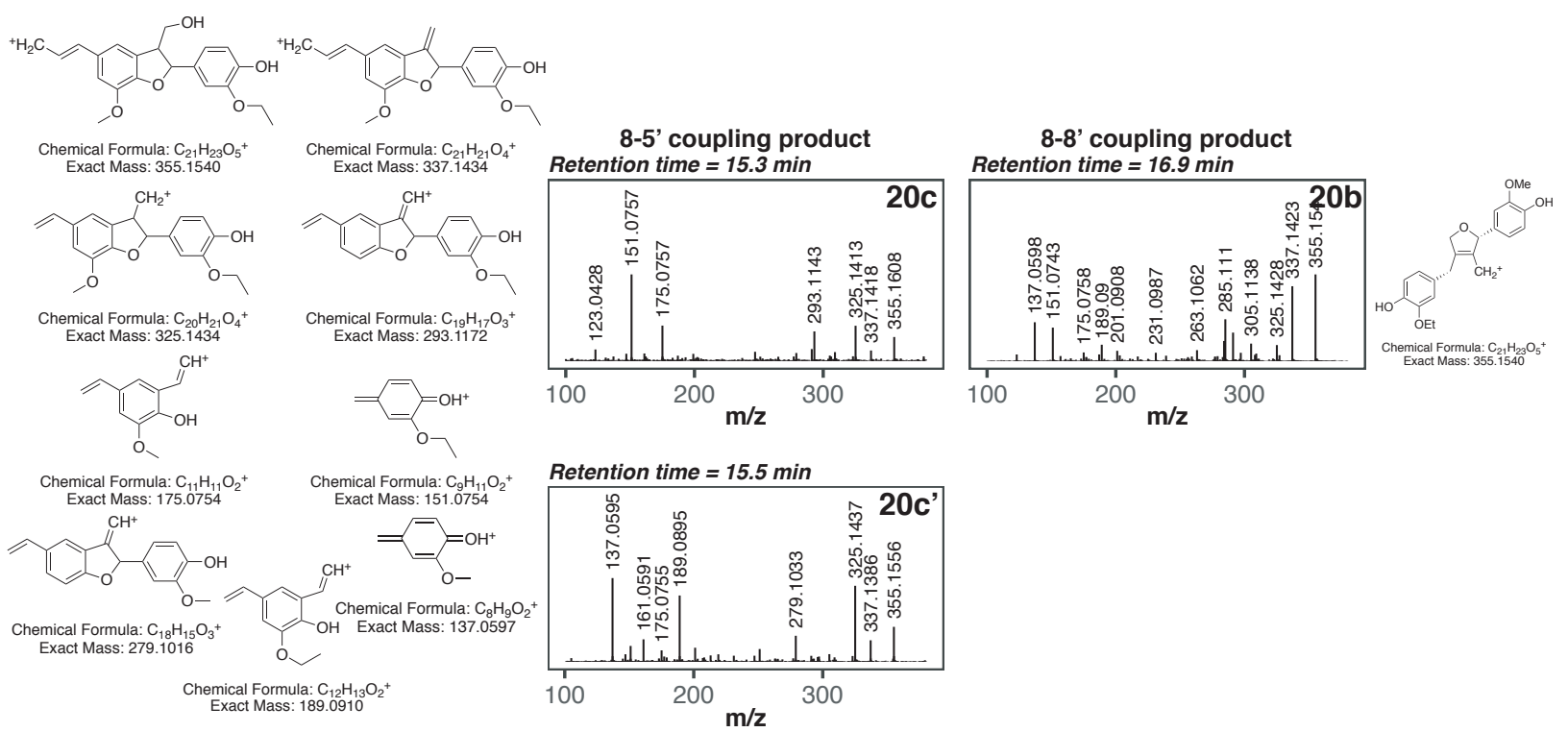

Figure S12. MS/MS fragmentation patterns support the structure assignment of 8-5' coupling products.

The precursor ions of the peaks designated as 8-5' and 8-8' coupling products as shown in Figure 3 were fragmented at the $\mathrm{m} / \mathrm{z}$ of $\left[\mathrm{M}-\mathrm{H}_{2} \mathrm{O}+\mathrm{H}\right]^{+}$for (a) 2, (b) 19, and (c) 20. Putative ion structures for the observed

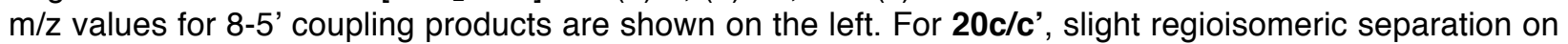
C18 chromatography allowed acquisition of two MS/MS spectra matching either regioisomer's expected fragmentation pattern. 
a
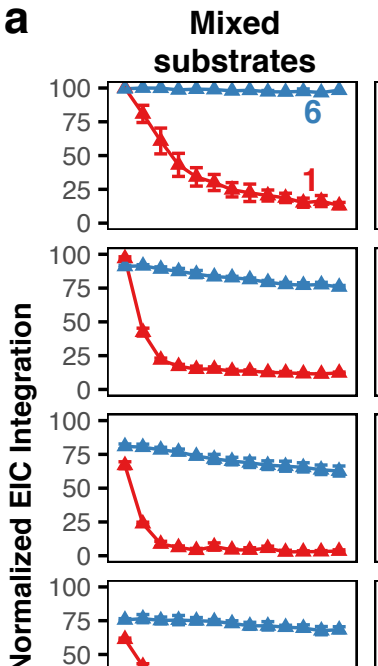

z
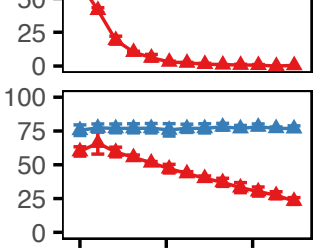

$$
0-
$$

Coupling products
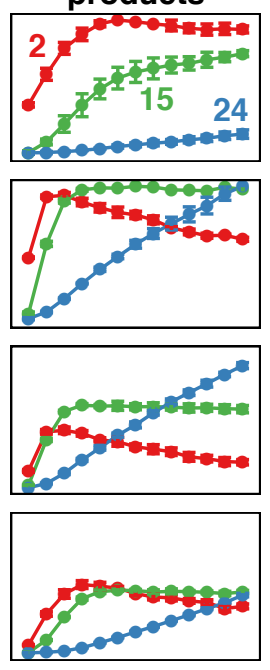

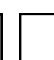

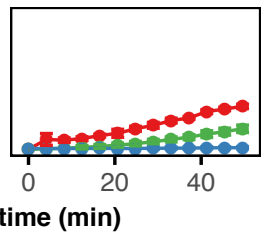

b

pH 2

pH 4

pH 5.5

pH 8.5

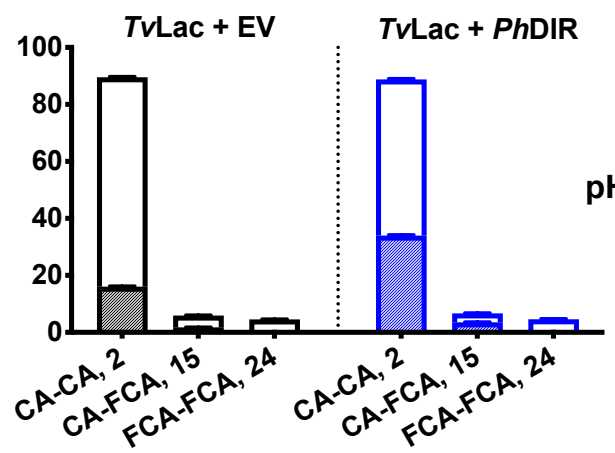

$\mathrm{pH} 4$

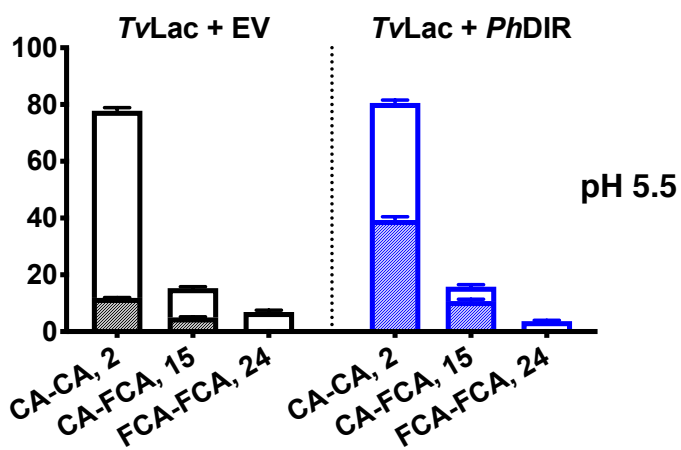

Figure S13. TULac oxidation of 1 and 6 (1:9 molar ratio) at various $\mathrm{pH}$ does not alter the relative oxidation rates.

(a) Time-course of TVLac oxidation of 1 and 6 at pH 2, 4, 5.5, 7, and 8.5. At any $\mathrm{pH}$ tested, the oxidation of 6 appears to be much slower than that of 1.

Data points indicate the mean of peak integrations for the corresponding chemical species ( $\mathrm{m} / \mathrm{z}$ of [M$\left.\mathrm{H}_{2} \mathrm{O}+\mathrm{H}\right]^{+}$) detected by LC-MS, normalized to the range of maximum and minimum peak integrations per chemical species across all reactions. Error bars show standard deviations of data from triplicate reactions. (b) End-point analysis of coupling of $\mathbf{1}$ and $\mathbf{6}$ with and without PhDIR. Regioselectivity improvement in the presence of $P h D I R$ is better at $\mathrm{pH} 5.5$ compared to $\mathrm{pH} 4$.

$\mathrm{Y}$-axis values are normalized the sum of total coupling product content per reaction as calculated by EIC integration of corresponding $\mathrm{m} / \mathrm{z}$ of $\left[\mathrm{M}-\mathrm{H}_{2} \mathrm{O}+\mathrm{H}\right]^{+}$, with $\mathrm{M}$ matching the exact mass of the 8-8' coupling product and its regioisomer, 8-5' coupling product. Filled, slash-patterned bars indicate the 8-8' coupling products ( $\mathbf{2 b}$ and $\mathbf{1 5 b}$ ). Bar heights indicate the mean of triplicates, and error bars standard deviations. 


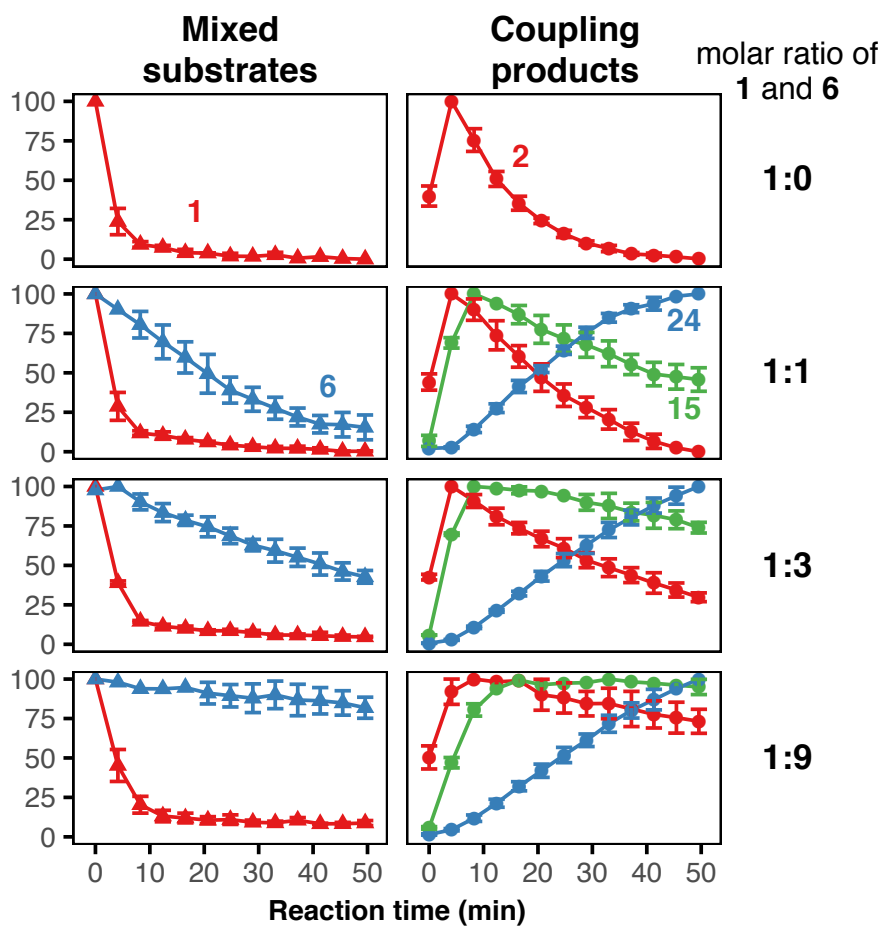

Figure S14. Time-course of TVLac oxidation of $\mathbf{1}$ and $\mathbf{6}$ at various ratios.

Data points indicate the mean of peak integrations for the corresponding chemical species ( $\mathrm{m} / \mathrm{z}$ of [M$\left.\mathrm{H}_{2} \mathrm{O}+\mathrm{H}\right]^{+}$) detected by LC-MS, normalized to the range of maximum level observed per reaction and minimum level (limit of detection) per chemical species across all reactions. Error bars show standard deviations of data from triplicate reactions. The data shown here are the same shown in Figure $\mathbf{4 b}$ with normalization per reaction to show the trends of substrate oxidation and product formation. 


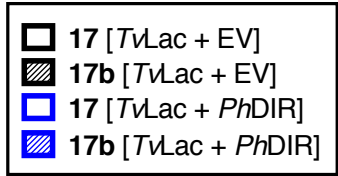

a

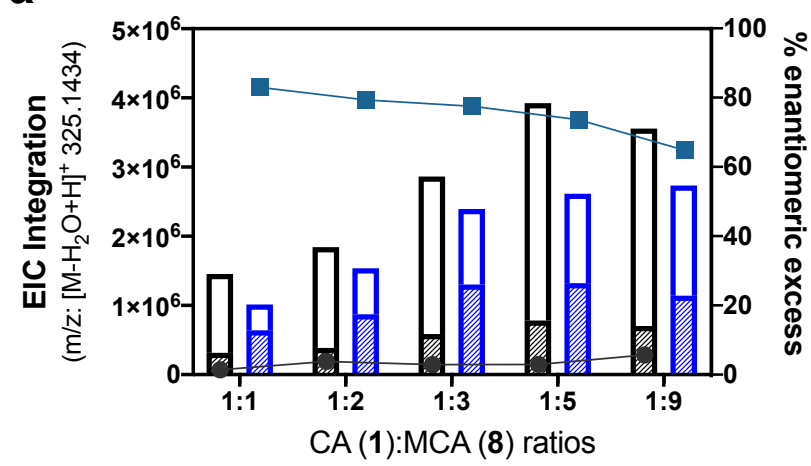

b

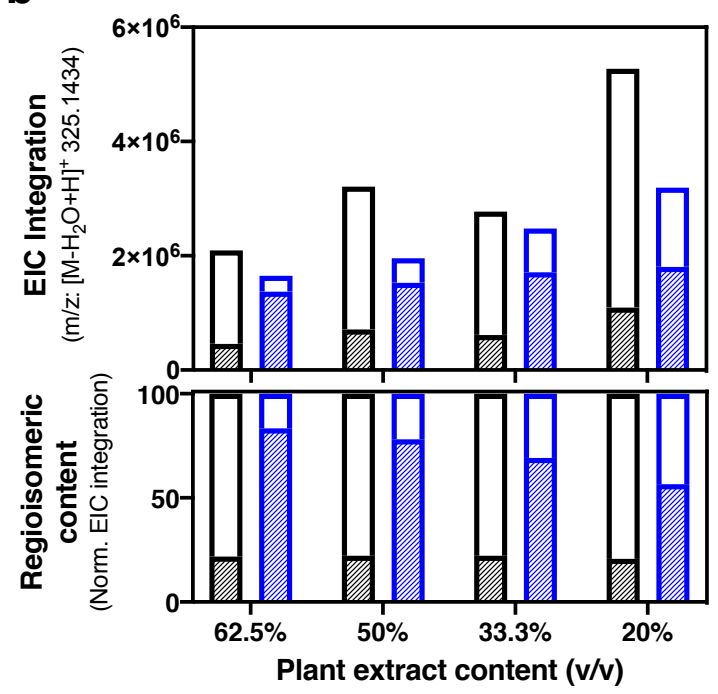

Figure S15. Optimization of PhDIR-mediated heterocoupling of 1 and 8

(a) With increasing amounts of 8 with respect to 1 (left to right on the x-axis), yield of the 8-8' heterocoupling product (17b) increases with a diminishing return (filled, slash-patterned bars), while regio- and enantioselectivity decrease due to the increased initial total substrate concentration.

Left $y$-axis values correspond to the bar heights indicating the total heterocoupling product (17) content per reaction based on the EIC integration on reverse-phase $\mathrm{C} 18$ chromatography, with $\mathrm{M}$ matching the exact mass of the 8-8' heterocoupling product and its regioisomer, 8-5' heterocoupling product. Filled, slashpatterned bars indicate the peak integration corresponding to the 8-8' heterocoupling product (17b).

Right $y$-axis values correspond to the data points indicating \% enantiomeric excess determined by chiral chromatography (circles: TVLac-EV, squares: TVLac- $P$ DDIR reactions).

(b) With increasing amounts of plant extracts expressing PhDIR (right to left on the x-axis, blue bars), regioselectivity increase is observed (relative content of the 8-8' heterocoupling product [17b] with respect to the total heterocoupling products 17), while the absolute yield (indicated by EIC integrations) of 17b remains unchanged. Some components present in the plant extracts (e.g. plant peroxidases, chloride concentration) might impact product accumulation.

A 1:3 (molar ratio) mixture of 1 and $\mathbf{8}$ was subjected to TVLac-EV (black) or TVLac-PhDIR (blue) reactions. $\mathrm{Y}$-axis values indicate the EIC integration of the heterocoupling products (17), with $\mathrm{M}$ matching the exact mass of the 8-8' heterocoupling product and its regioisomer, 8-5' heterocoupling product. Filled, slashpatterned bars indicate the peak integration corresponding to the 8-8' heterocoupling product (17b). 


\section{a. coupling of 1 and 10}

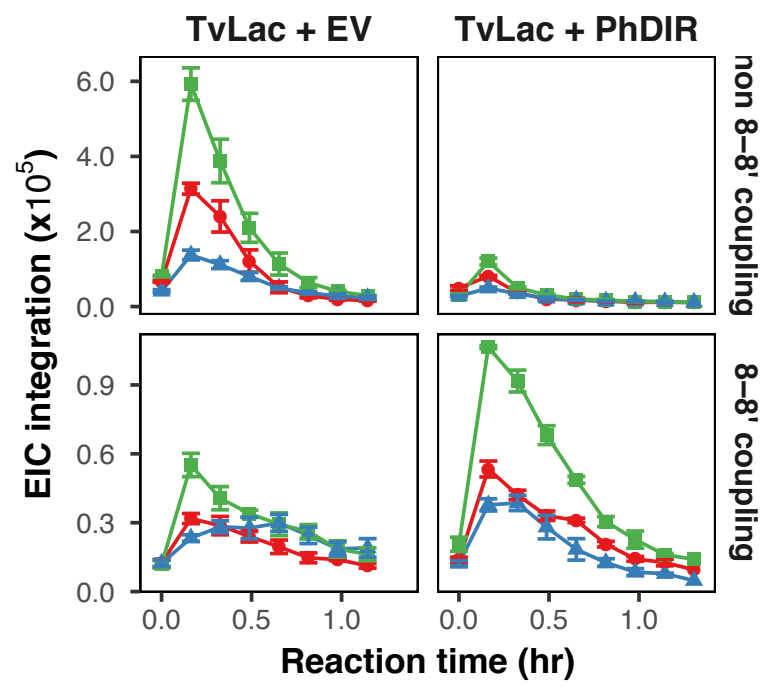

$-2-28-19$

\section{b. coupling of 1 and 11}

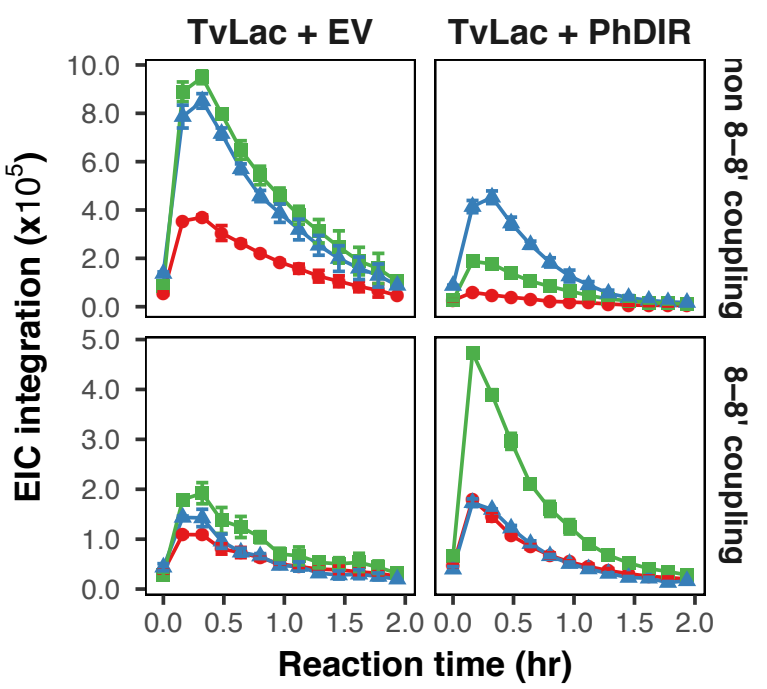

$-2-29-20$

Figure S16. Time-course of oxidative coupling reactions by TVLac in absence or presence of PhDIR The coupling products resulting from all coupling modes get further oxidized by TVLac (as indicated by decreasing trends). The TVLac-alone reaction was balanced with the plant extract from EV-expressing $N$. benthamiana leaves to account for undetermined levels of native oxidants and ion species (notably chlorides acting as a laccase inhibitor) present in the PhDIR extract.

$\mathrm{Y}$-axis values show coupling product levels estimated from integration of EIC peaks at $\mathrm{m} / \mathrm{z}$ of $\left[\mathrm{M}-\mathrm{H}_{2} \mathrm{O}+\mathrm{H}\right]^{+}$ of the corresponding product, with $\mathrm{M}$ matching the exact mass of an 8-8' coupling product and its regioisomer, 8-5' coupling product. Out of the three major coupling modes (8-0-4', 8-5', and 8-8'; see Scheme 1), 8-O-4' coupling product is heavier than the other two due to water addition. Thus, peaks observed at the ion mass of $\left[\mathrm{M}-\mathrm{H}_{2} \mathrm{O}+\mathrm{H}\right]^{+}$is mostly 8-8' and 8-5' coupling products and in-source fragmentation products of 8-O-4' coupling products (the latter two comprise the majority of "non 8-8' coupling" product levels). Data points indicate the mean of triplicates, and error bars standard deviations. 


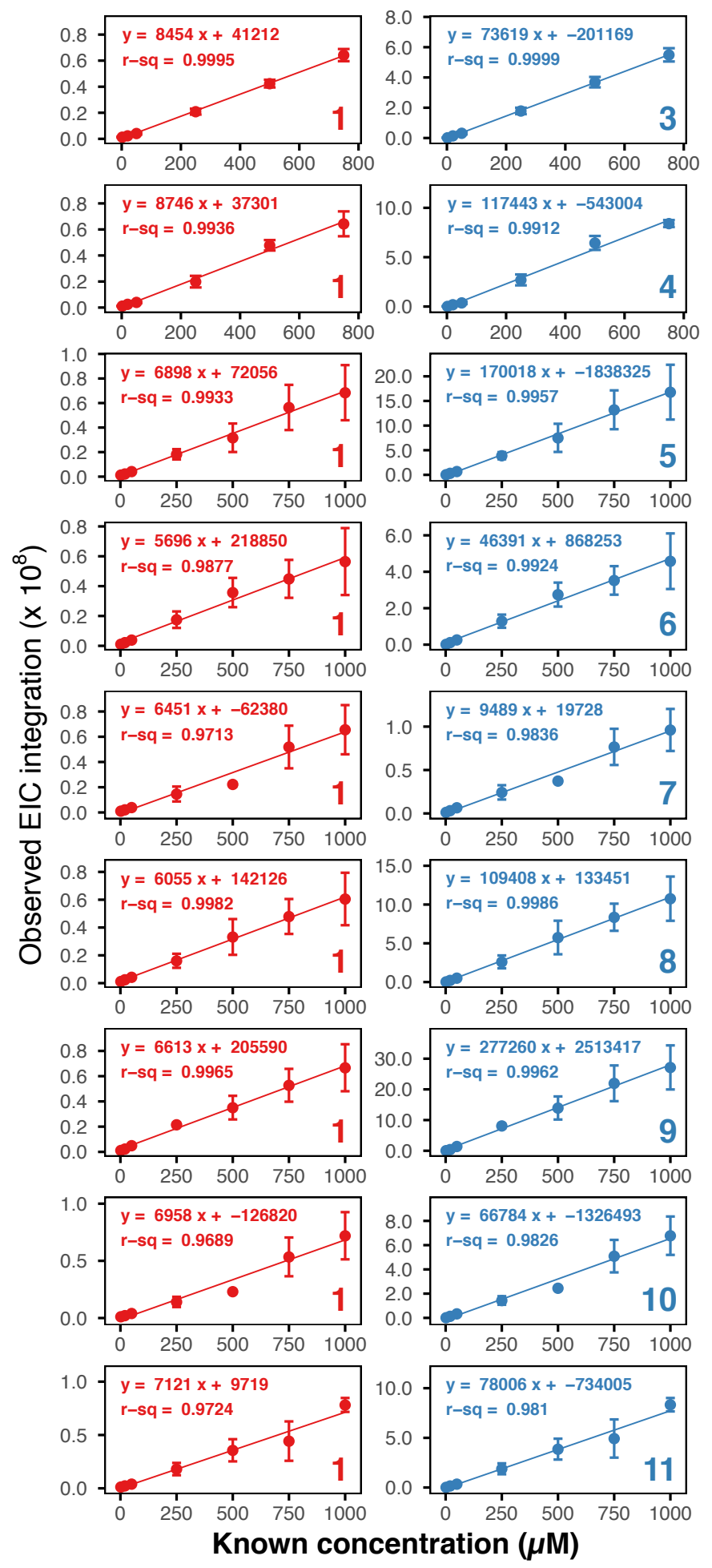

Figure S17. Standard curves of monolignols in TVLac oxidation assays

An equimolar mixture of 1 and an analog shows linear response detected by LC-MS (m/z of $\left.\left[\mathrm{M}-\mathrm{H}_{2} \mathrm{O}+\mathrm{H}\right]^{+}\right)$ over the concentration range of $5 \mu \mathrm{M}$ to 800 or $1000 \mu \mathrm{M}$. Each row depicts the equimolar mixture, mimicking the equimolar-substrate TVLac oxidation reaction conditions, with BSA supplemented to replace TVLac in MES buffer at pH 5.5. Data points indicate the mean integrations (area under the EIC curve) of triplicates, and error bars standard deviations. 
a

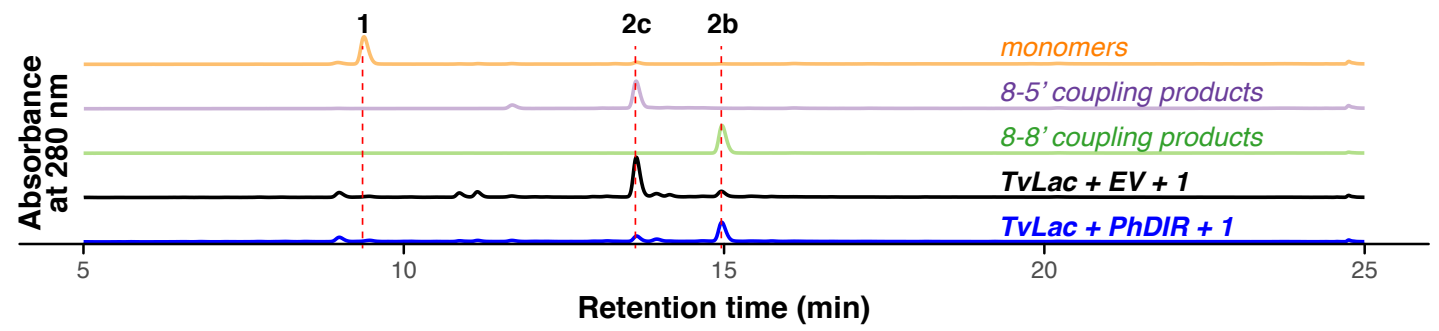

b

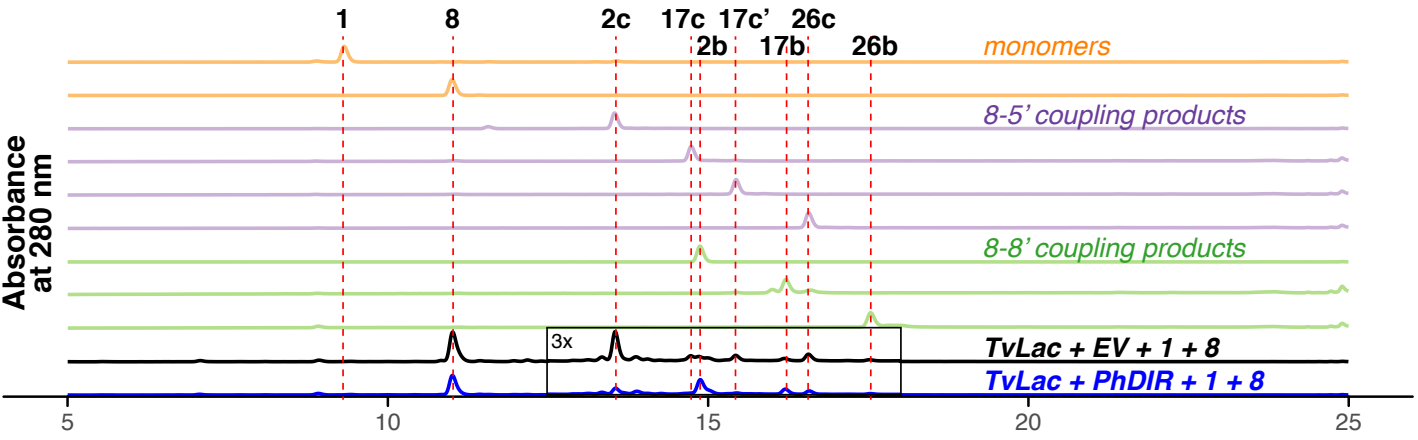

C

Retention time (min)

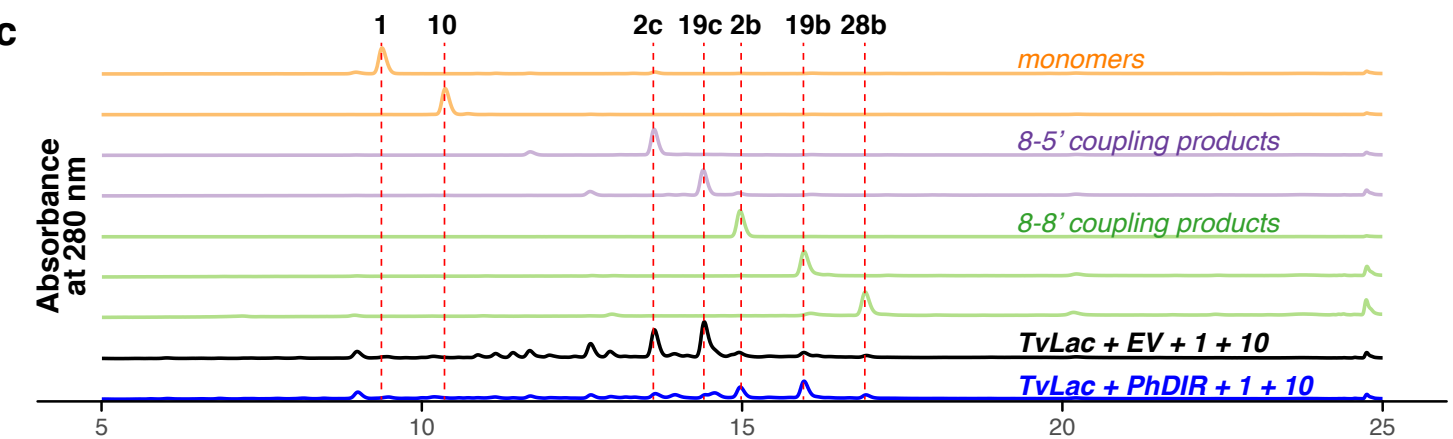

Retention time (min)

d

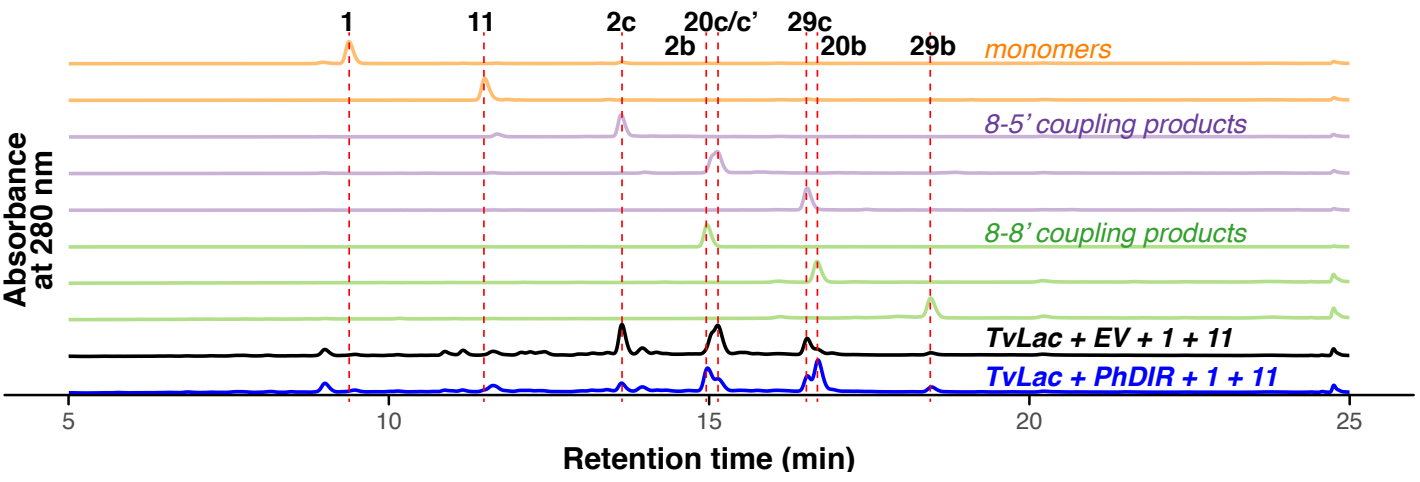



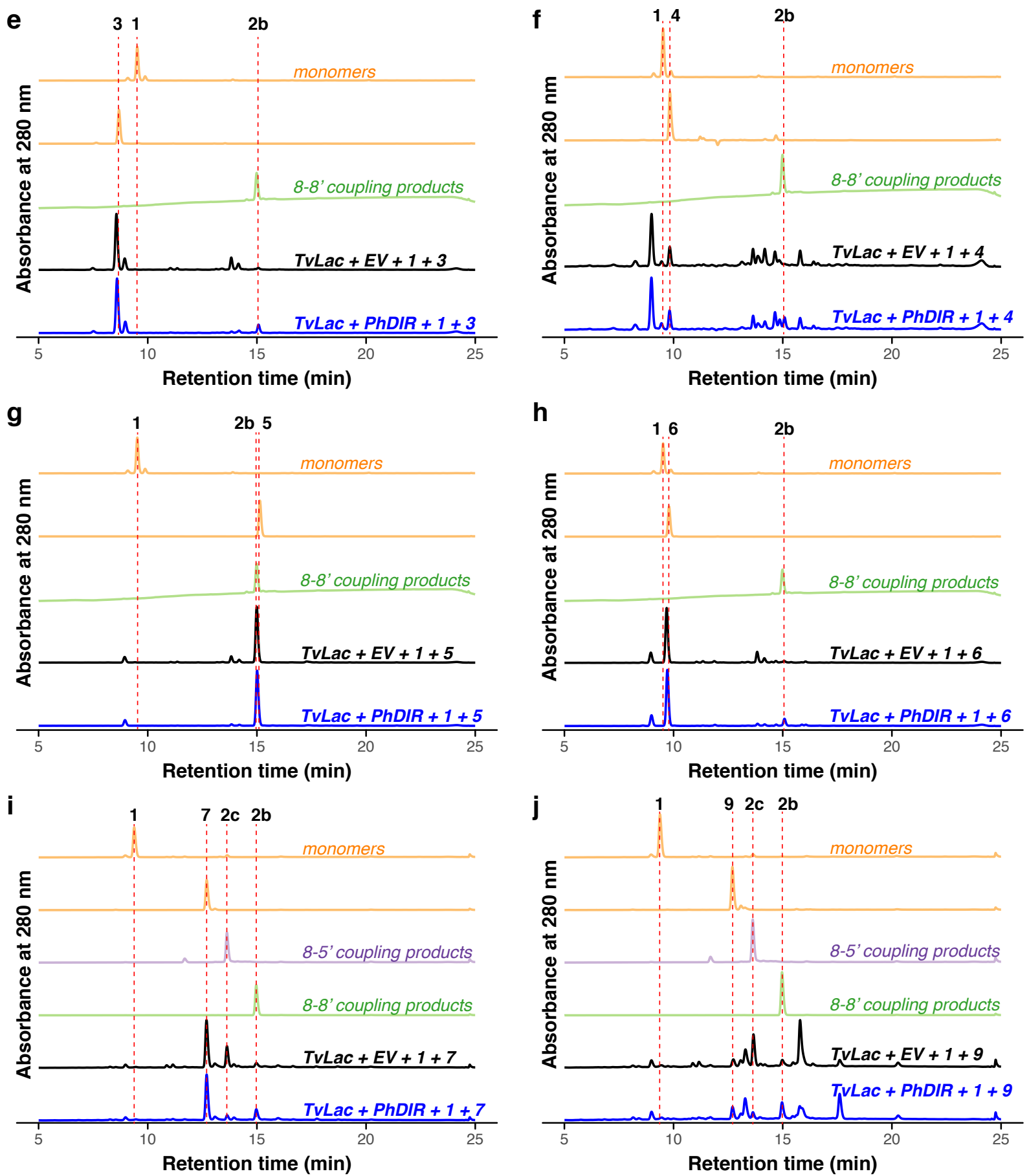

Figure S18. Representative full UV chromatograms of coupling reactions catalyzed by TVLac and mediated by $P h D I R$

Coupling product distributions for these reactions are reported in Figures $\mathbf{2}$ and S2. Compound $\mathbf{1}$ can be coupled with a) itself (native reaction), b) $\mathbf{8}$, c) 10, d) 11, e) 3, f) 4, g) 5, h) 6, i) $\mathbf{7}$, or j) $\mathbf{9}$. Reactions were quenched at the point of full consumption for at least one of the two substrates (20 min for coupling of 1 and 4, and $40 \mathrm{~min}$ for all else). Sample traces (black and blue) are to scale to each other (unless otherwise noted), and the authentic standard traces (orange: monomers, lavender: 8-5' coupling products, and green: 8-8' coupling products) are normalized to fit scale. 
Table S1. Slopes and y-intercepts of linear equations from the standard curves

\begin{tabular}{rcc}
\hline Compound & $\begin{array}{c}\text { slope } \\
(\text { EIC integration } \mu \mathrm{M})\end{array}$ & $\begin{array}{c}\text { y-intercept } \\
\text { (EIC integration) }\end{array}$ \\
\hline (+)-2b & 25655 & 89797 \\
2c & 58019 & -106748 \\
$(+)-19 b$ & 42637 & 298016 \\
$\mathbf{1 9 c}$ & 55179 & 54469 \\
$(+)-20 b$ & 97766 & 886987 \\
20c/c' & 81215 & 102701 \\
$(+)-28 b$ & 27296 & 155244 \\
$(+)-29 b$ & 169969 & 2616120 \\
29c & 152459 & -293581 \\
\hline
\end{tabular}

The homocoupling products of 11 (29b and 29c) showed notably higher slopes than heterocoupling products of 1 and 11 (20b and $20 c / c^{\prime}$ ) or $2 b$ and $2 c$, indicating greater ionization efficiency (thus higher sensitivity) for the ethoxy-moiety containing chemical species at a given concentration. 


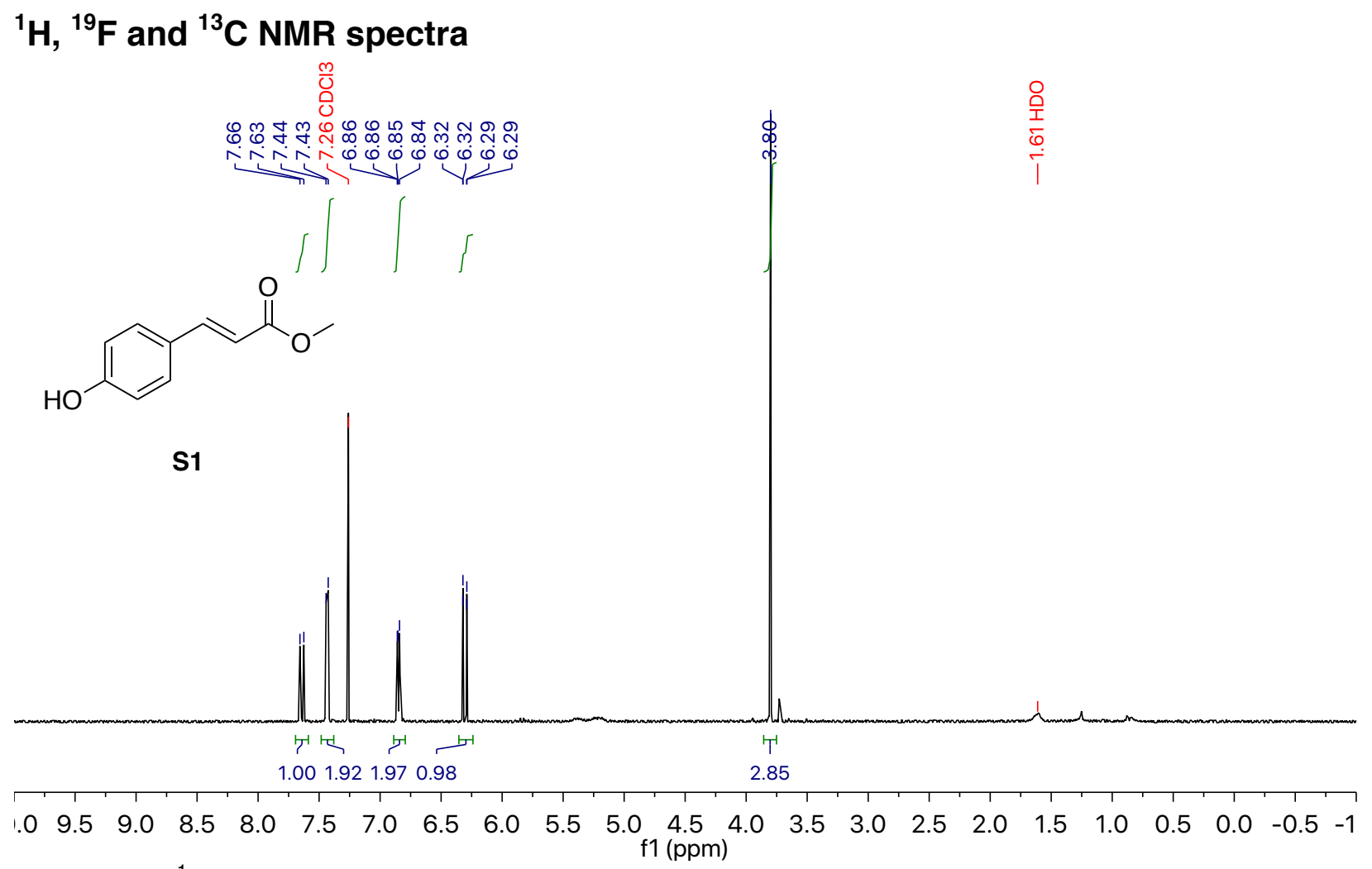

Figure S19. ${ }^{1} \mathrm{H}$ NMR spectrum of crude $\mathrm{S} 1$ in $\mathrm{CDCl}_{3}$ 

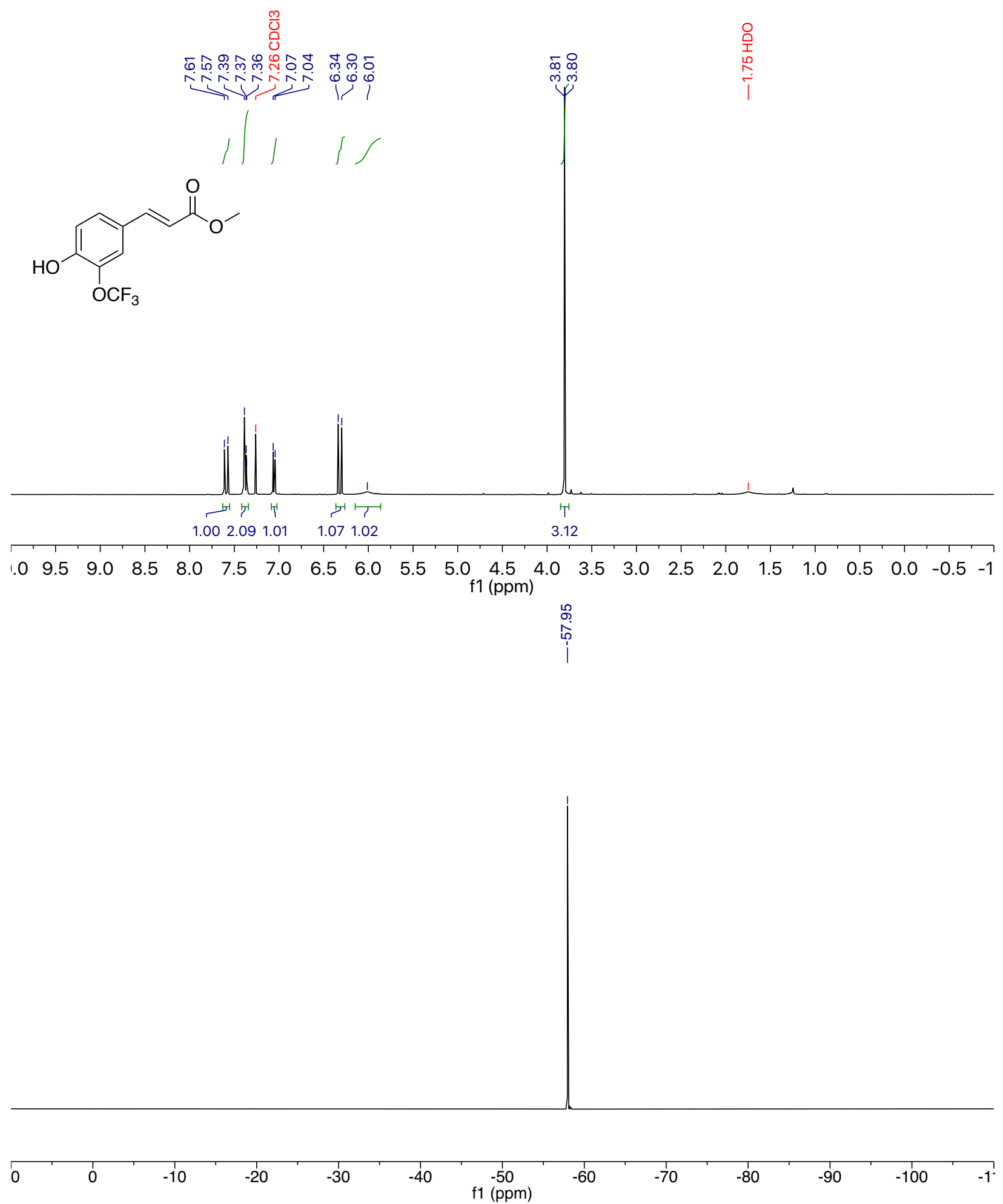


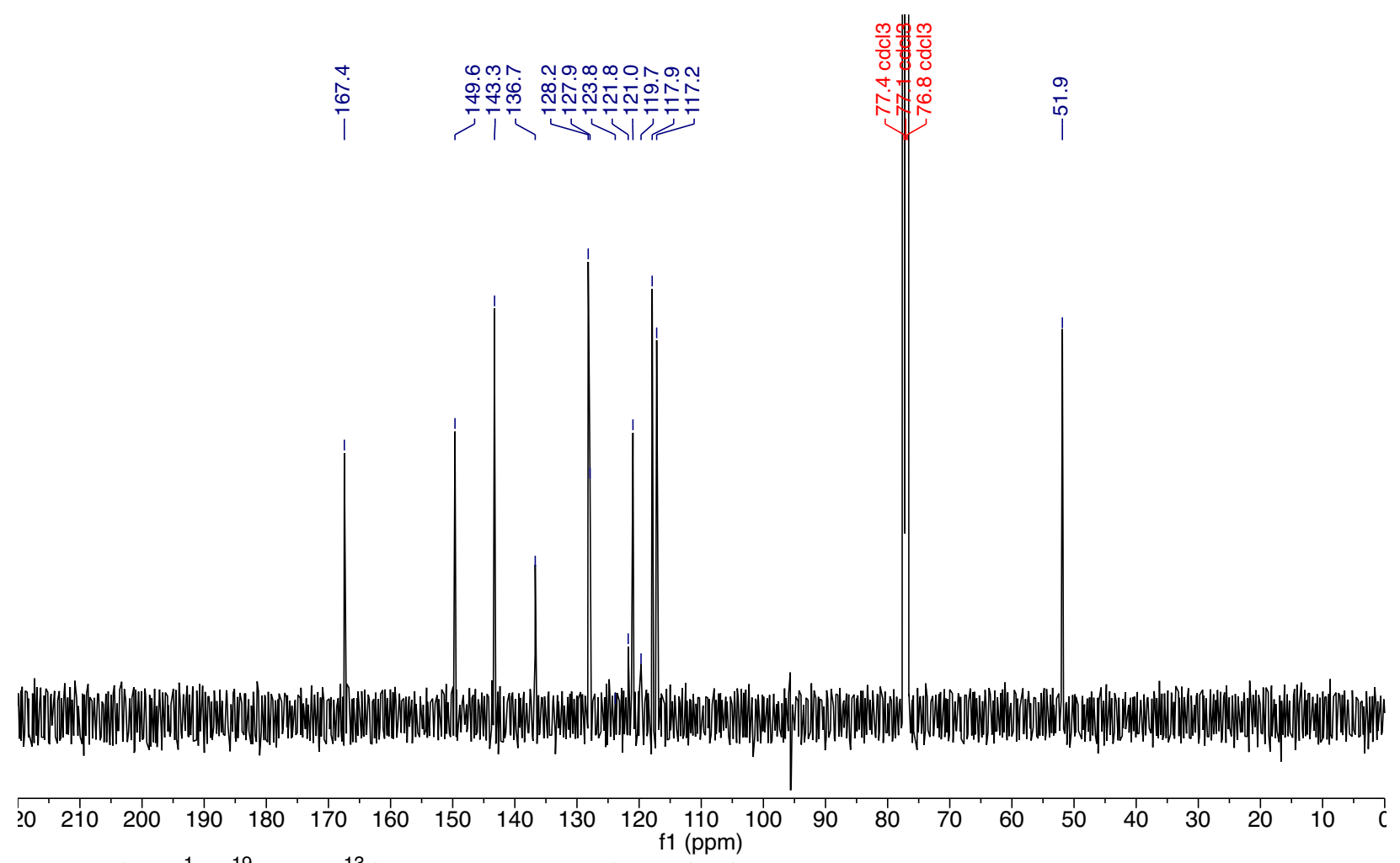

Figure S20. ${ }^{1} \mathrm{H},{ }^{19} \mathrm{~F}$, and ${ }^{13} \mathrm{C}$ NMR spectra of $\mathbf{S 2}$ in $\mathrm{CDCl}_{3}$ 


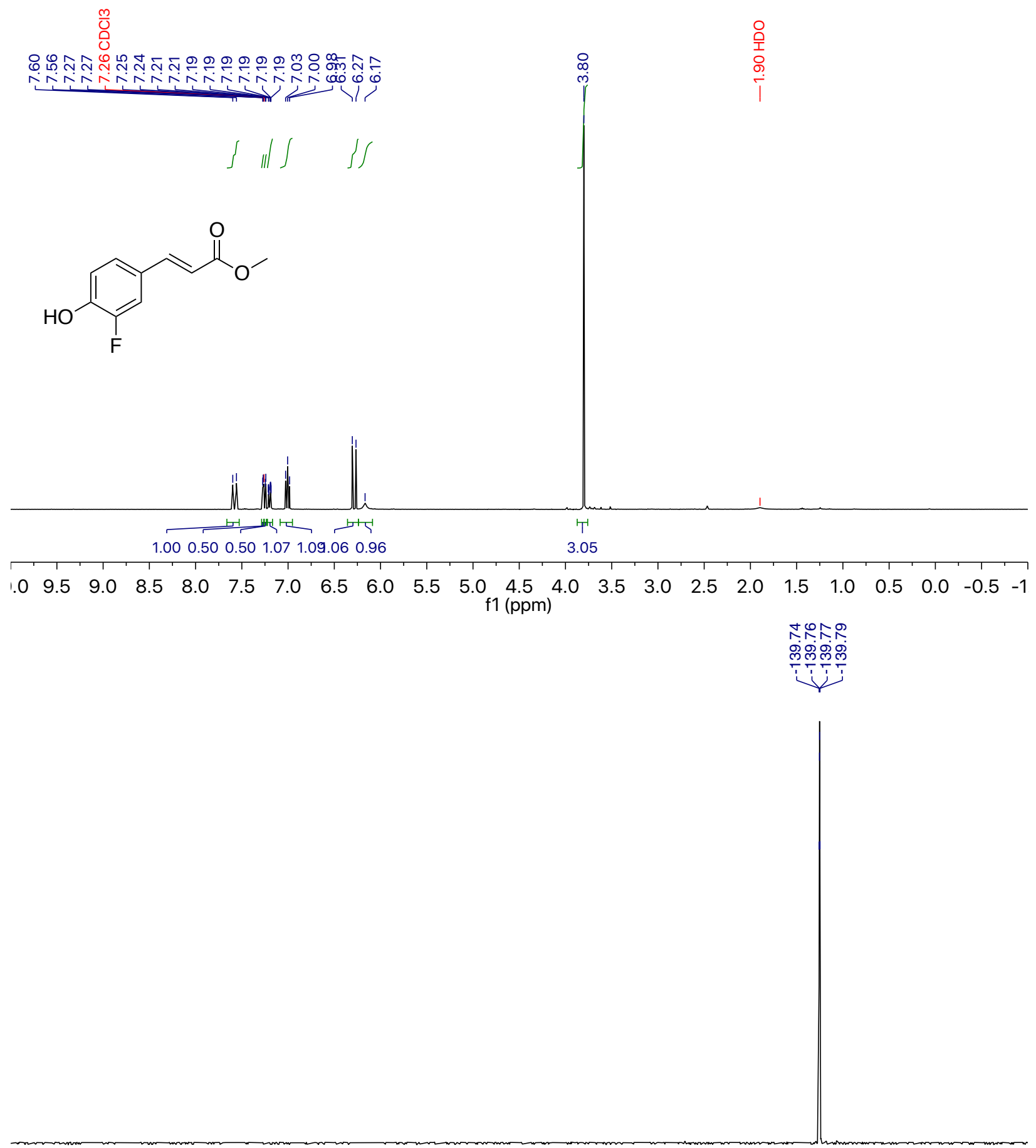

\begin{tabular}{|c|c|c|c|c|c|c|c|c|c|}
\hline 00 & -105 & -110 & -115 & -120 & $\begin{array}{c}-125 \\
\mathrm{f} 1(\mathrm{ppm})\end{array}$ & -130 & -135 & -140 & -145 \\
\hline
\end{tabular}




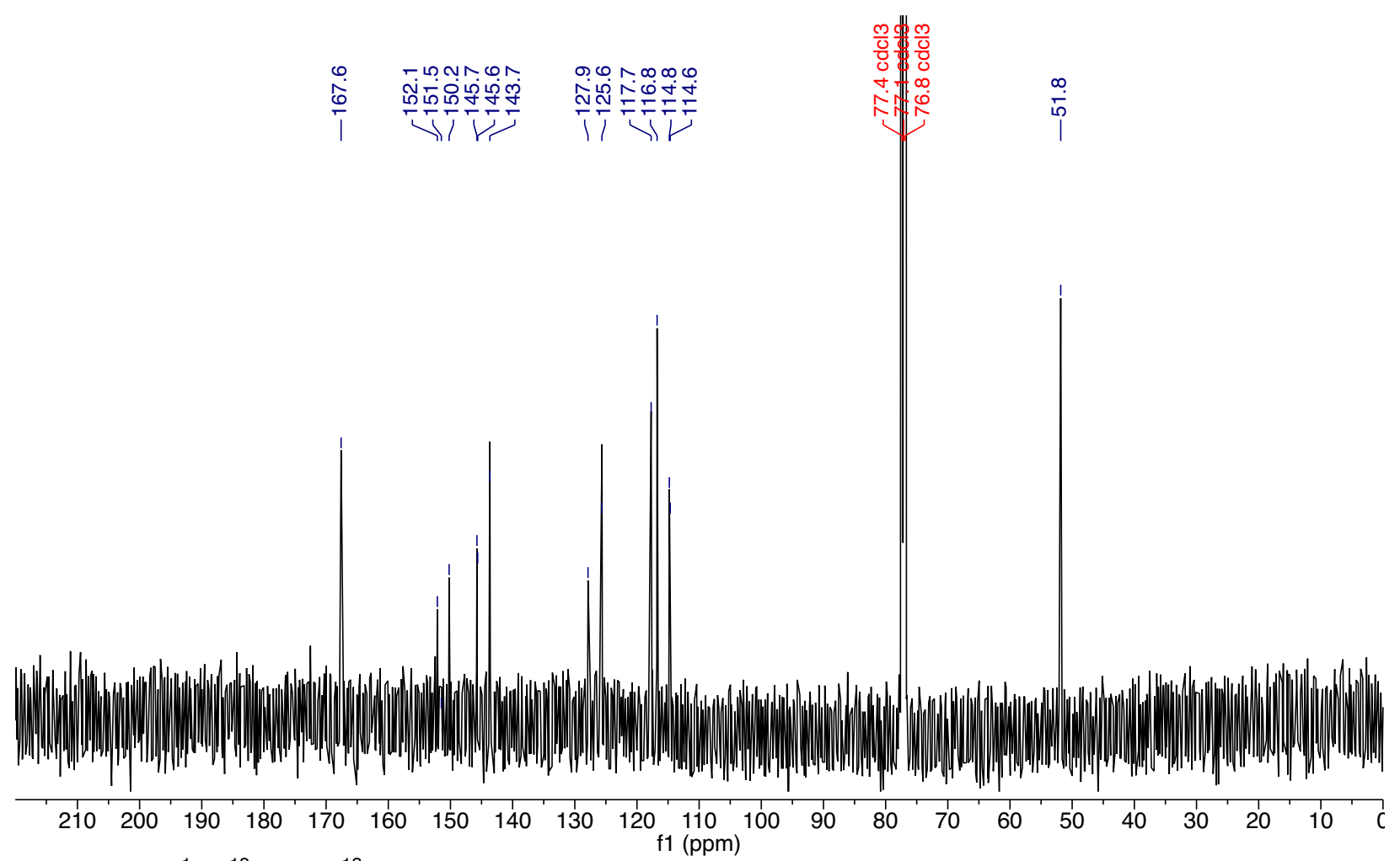

Figure $\mathbf{S 2 1} .{ }^{1} \mathrm{H},{ }^{19} \mathrm{~F}$, and ${ }^{13} \mathrm{C}$ NMR spectra of $\mathbf{S} 3$ in $\mathrm{CDCl}_{3}$ 


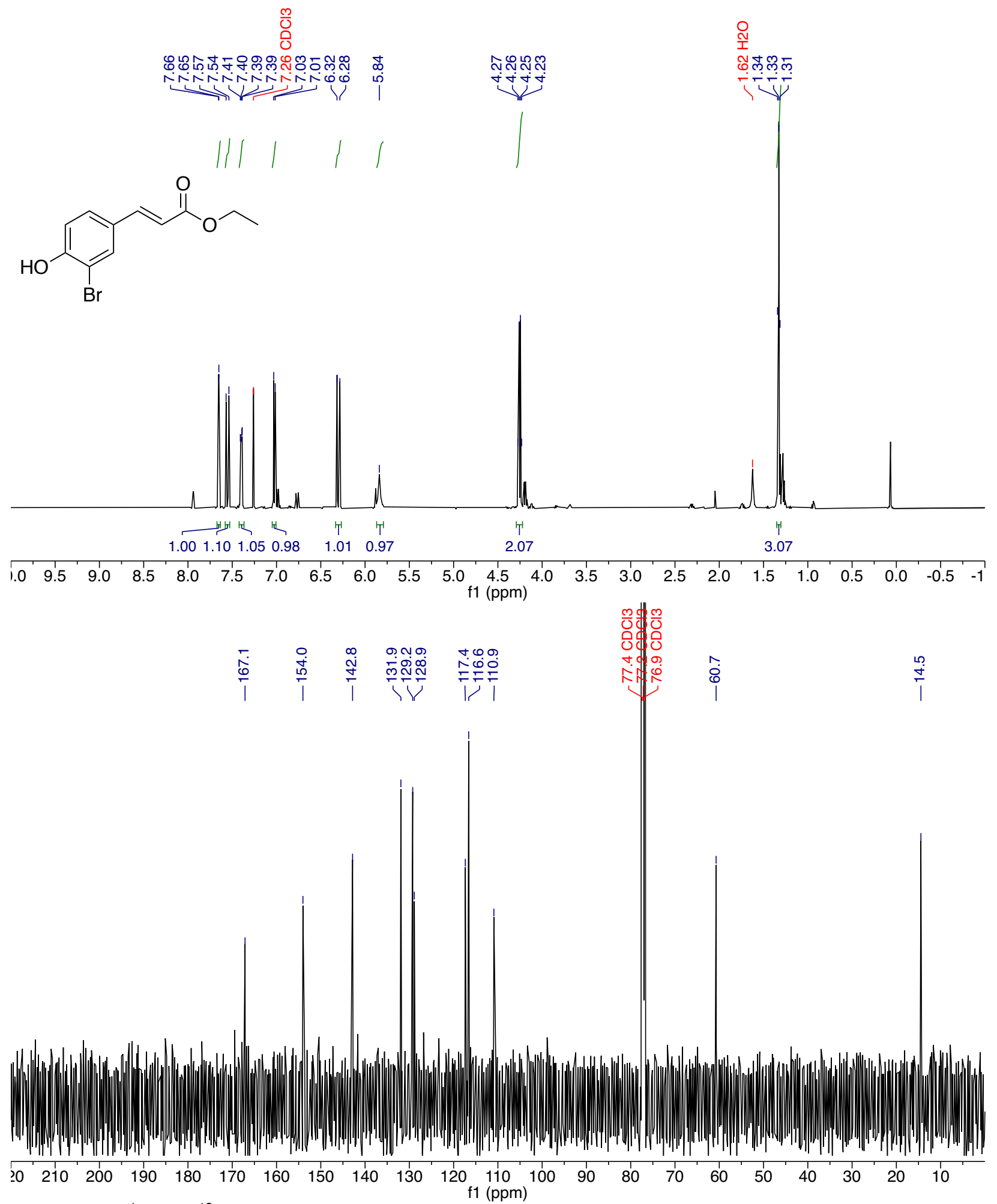

Figure S22. ${ }^{1} \mathrm{H}$ and ${ }^{13} \mathrm{C}$ NMR spectra of $\mathrm{S} 4 \mathrm{in} \mathrm{CDCl}_{3}$ 


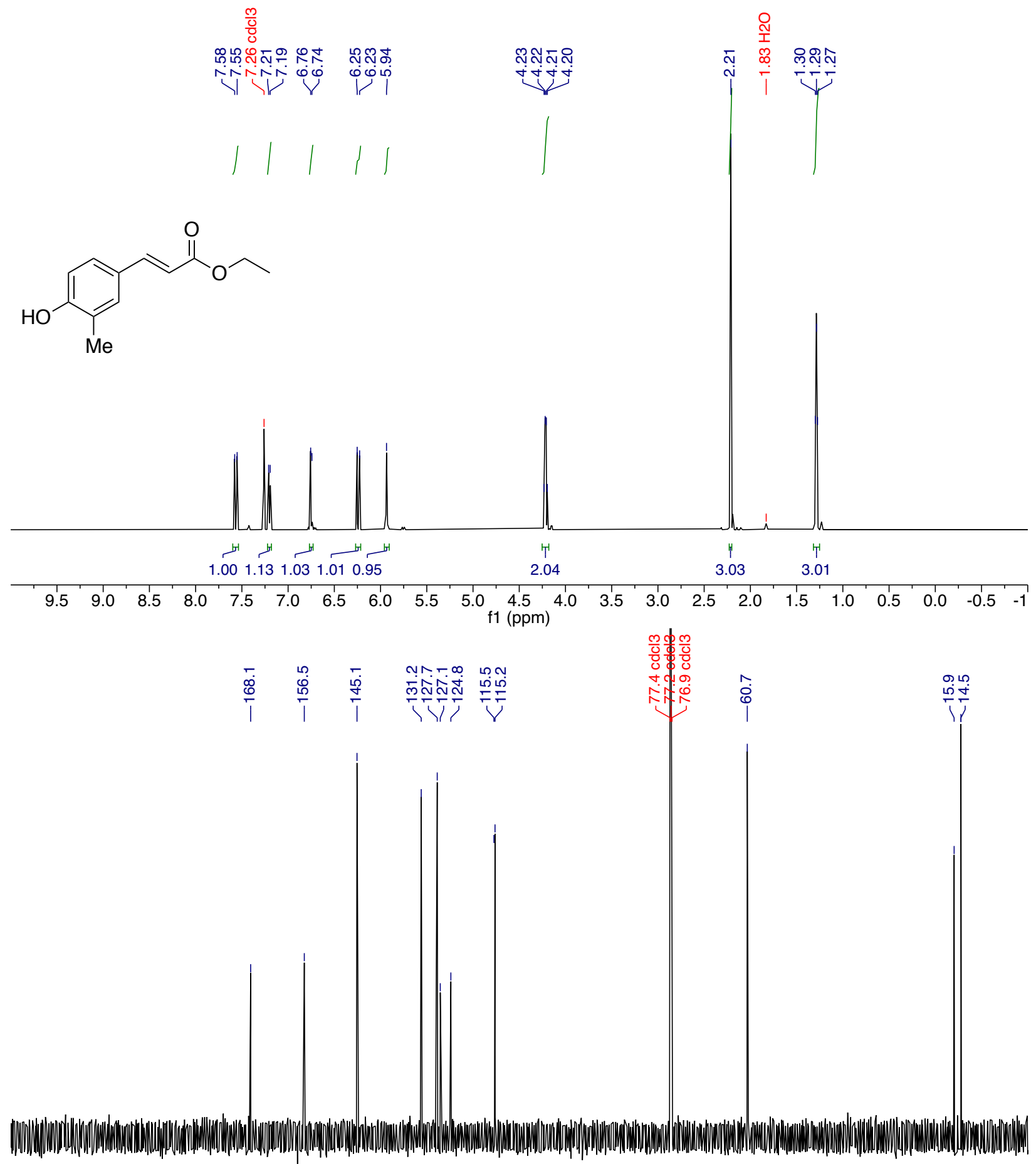

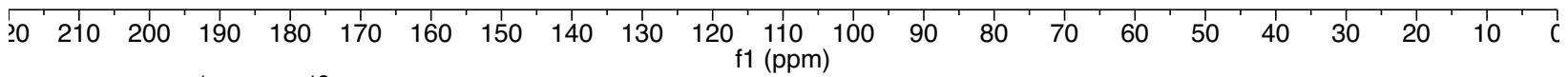

Figure S23. ${ }^{1} \mathrm{H}$ and ${ }^{13} \mathrm{C}$ NMR spectra of $\mathrm{S} 5$ in $\mathrm{CDCl}_{3}$ 


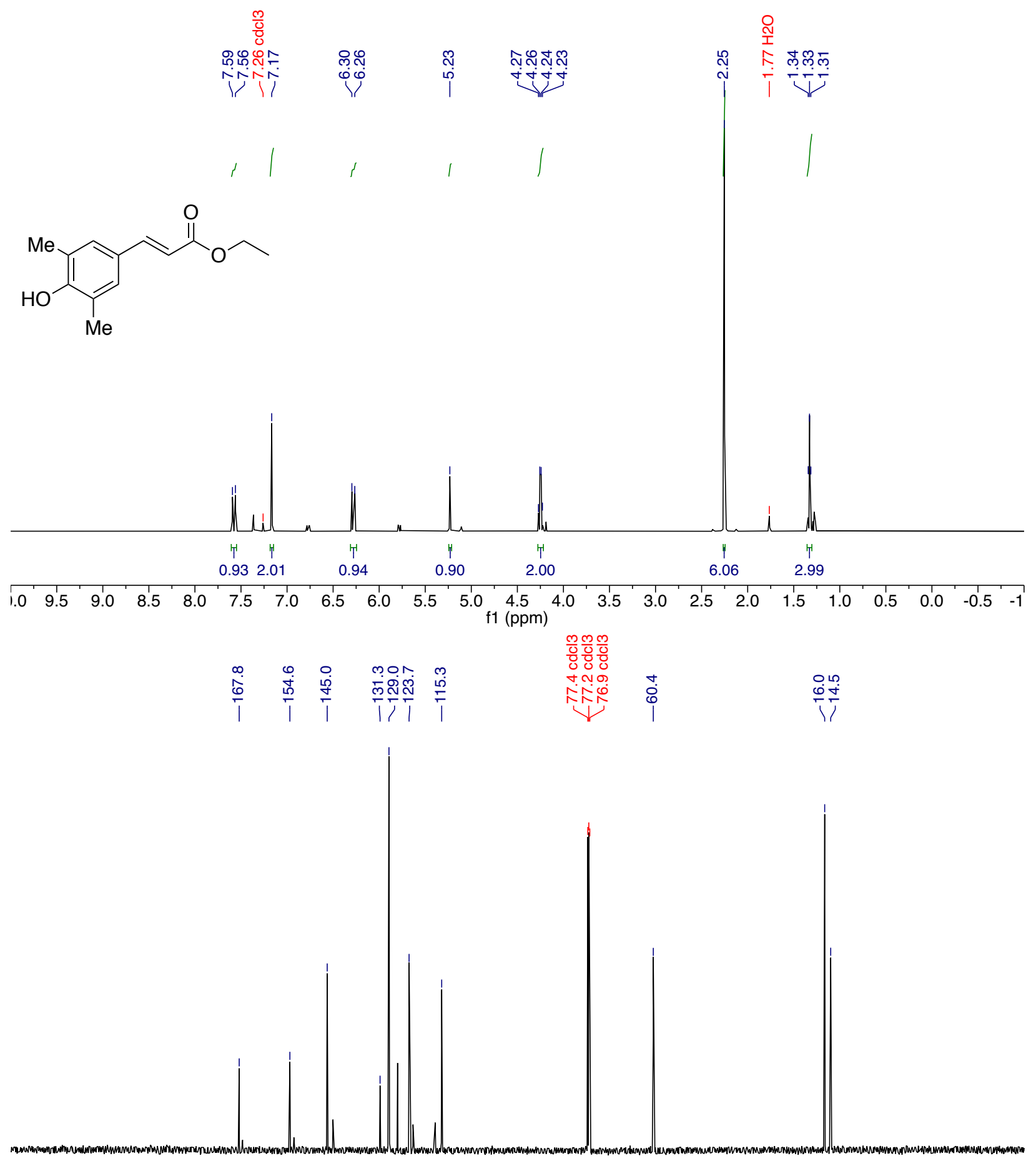

$\begin{array}{lllllllllllllllllllllllllllll}220 & 210 & 200 & 190 & 180 & 170 & 160 & 150 & 140 & 130 & 120 & 110 & 100 & 90 & 80 & 70 & 60 & 50 & 40 & 30 & 20 & 10 & 0 & -10 & -20 & -30\end{array}$

Figure S24. ${ }^{1} \mathrm{H}$ and ${ }^{13} \mathrm{C}$ NMR spectra of $\mathbf{S} 6$ in $\mathrm{CDCl}_{3}$ 


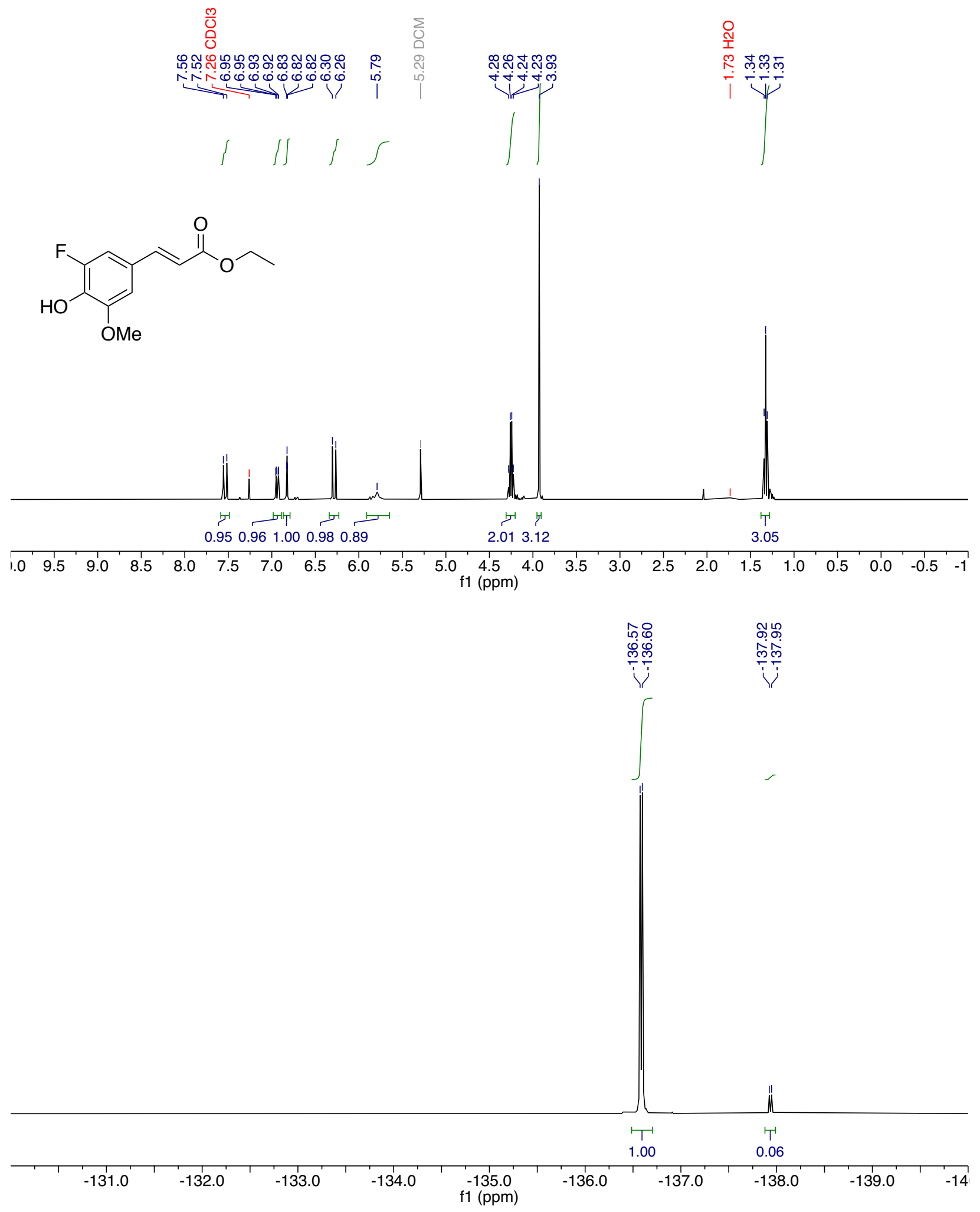




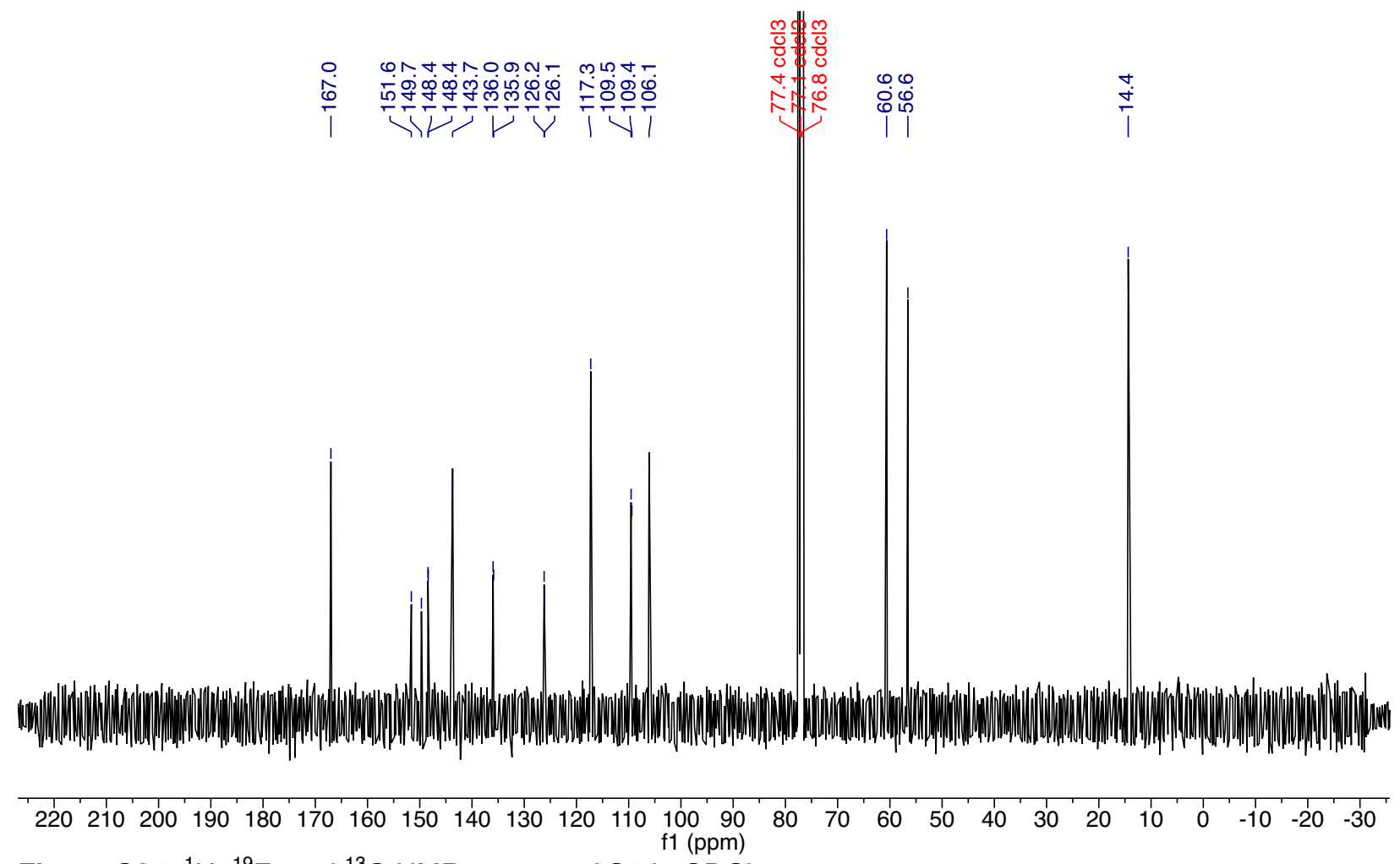

Figure S25. ${ }^{1} \mathrm{H},{ }^{19} \mathrm{~F}$, and ${ }^{13} \mathrm{C}$ NMR spectra of $\mathrm{S} 7 \mathrm{in} \mathrm{CDCl}_{3}$ 

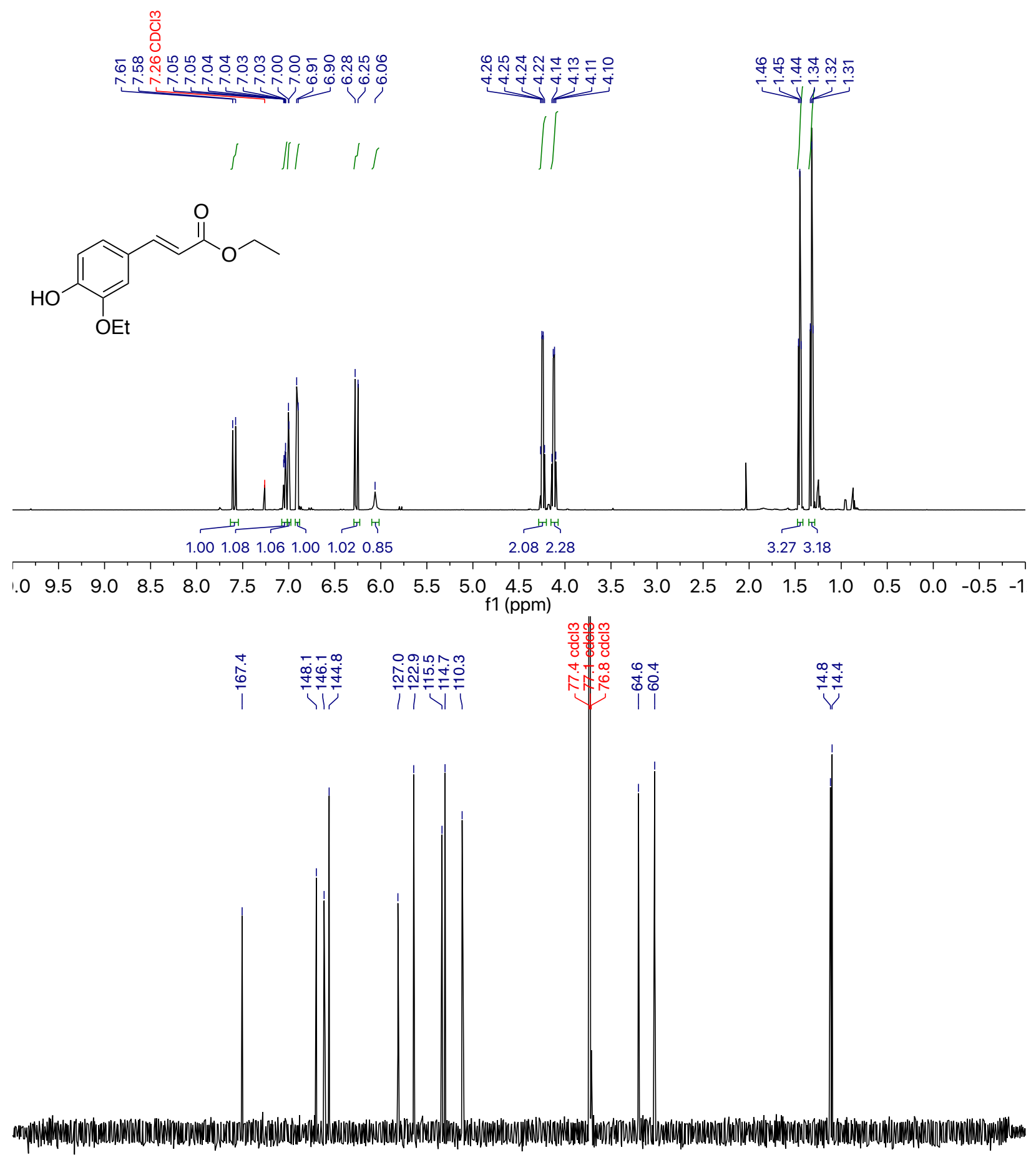

$\begin{array}{llllllllllllllllllllllllllllll}220 & 210 & 200 & 190 & 180 & 170 & 160 & 150 & 140 & 130 & 120 & 110 & 100 & 90 & 80 & 70 & 60 & 50 & 40 & 30 & 20 & 10 & 0 & -10 & -20 & -30\end{array}$

Figure S26. ${ }^{1} \mathrm{H}$ and ${ }^{13} \mathrm{C}$ NMR spectra of $\mathbf{S 8}$ in $\mathrm{CDCl}_{3}$ 


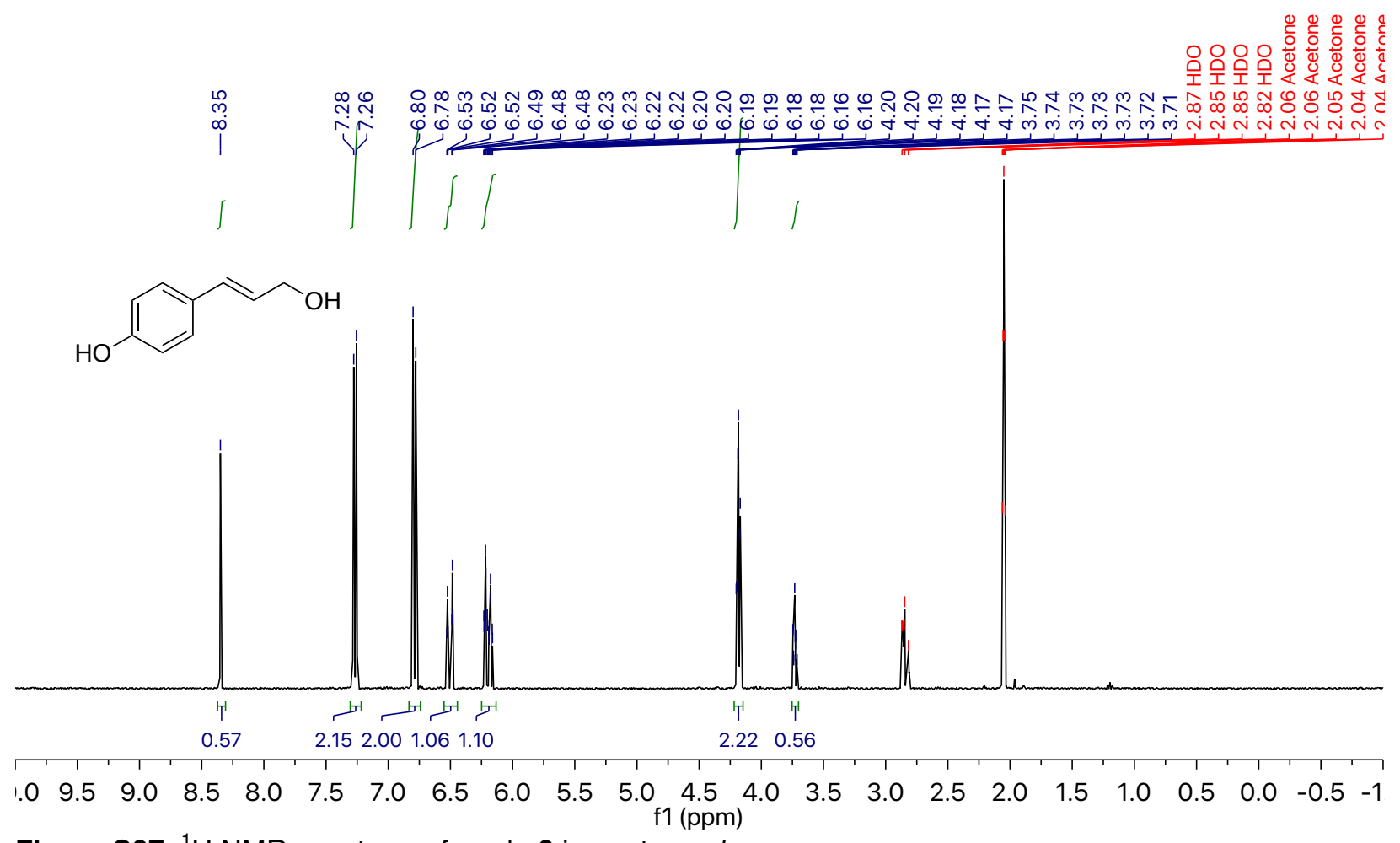

Figure S27. ${ }^{1} \mathrm{H}$ NMR spectrum of crude 3 in acetone- $d_{6}$ 

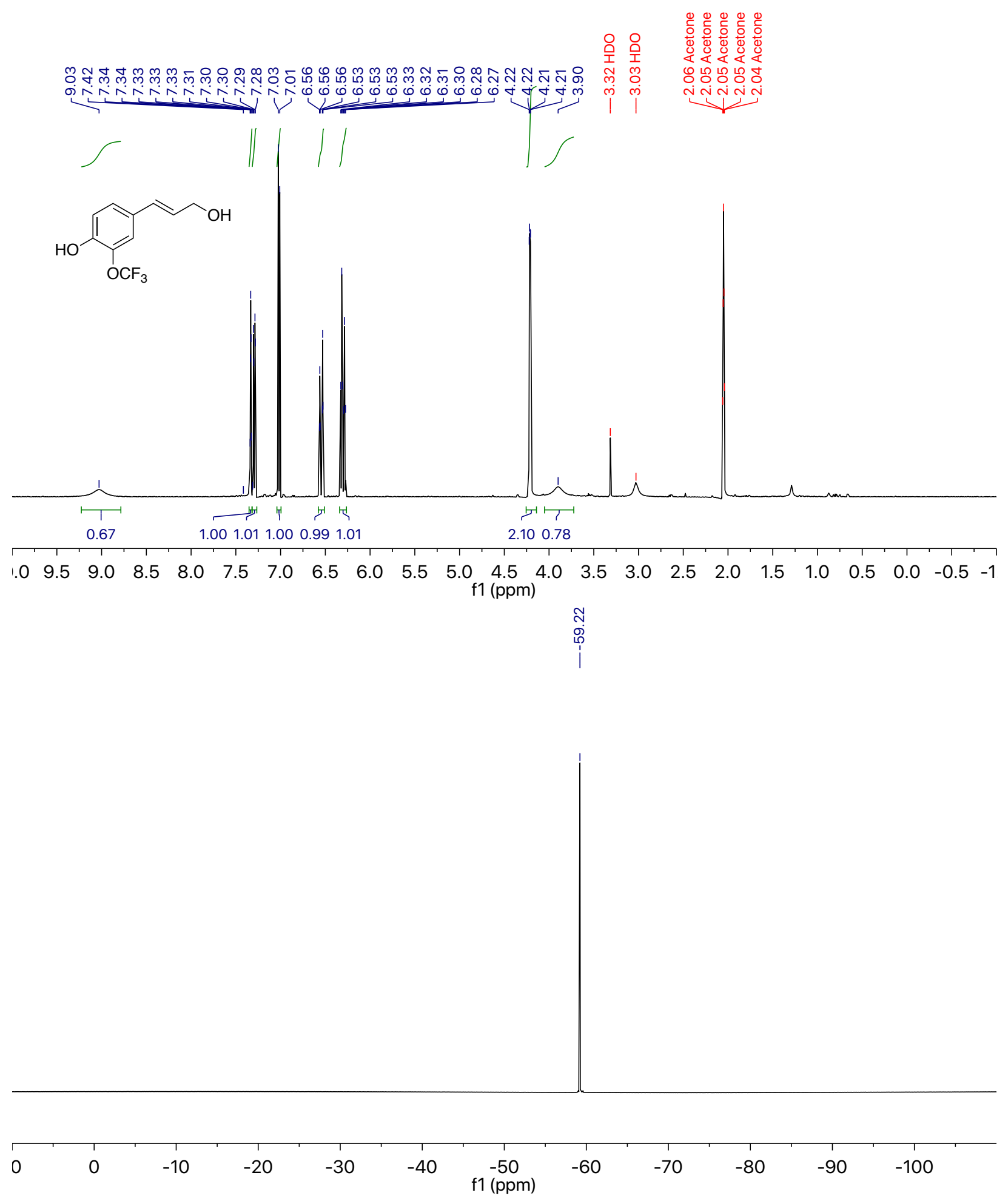

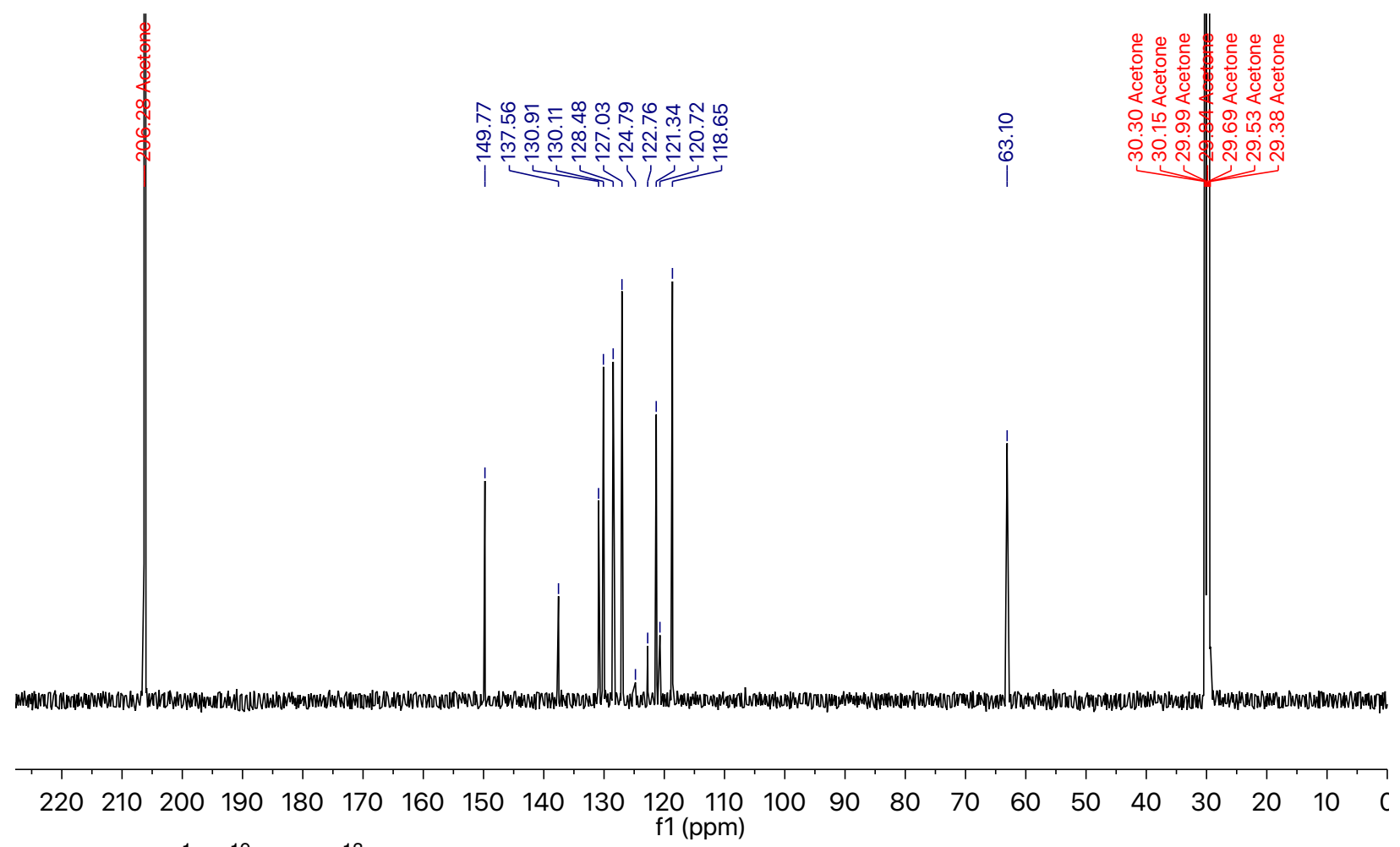

Figure S28. ${ }^{1} \mathrm{H},{ }^{19} \mathrm{~F}$, and ${ }^{13} \mathrm{C}$ NMR spectra of $\mathbf{5}$ in acetone- $d_{6}$ 


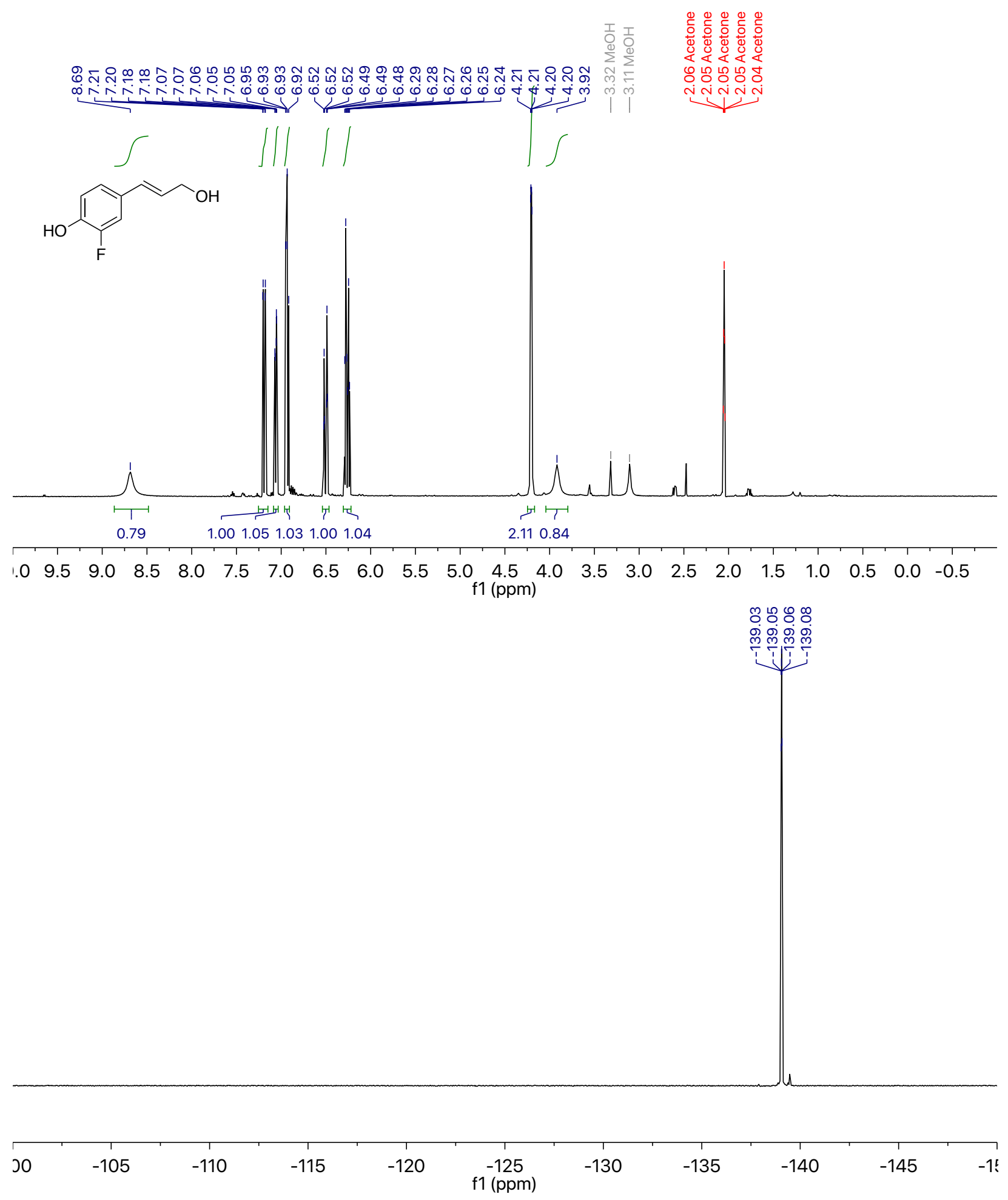



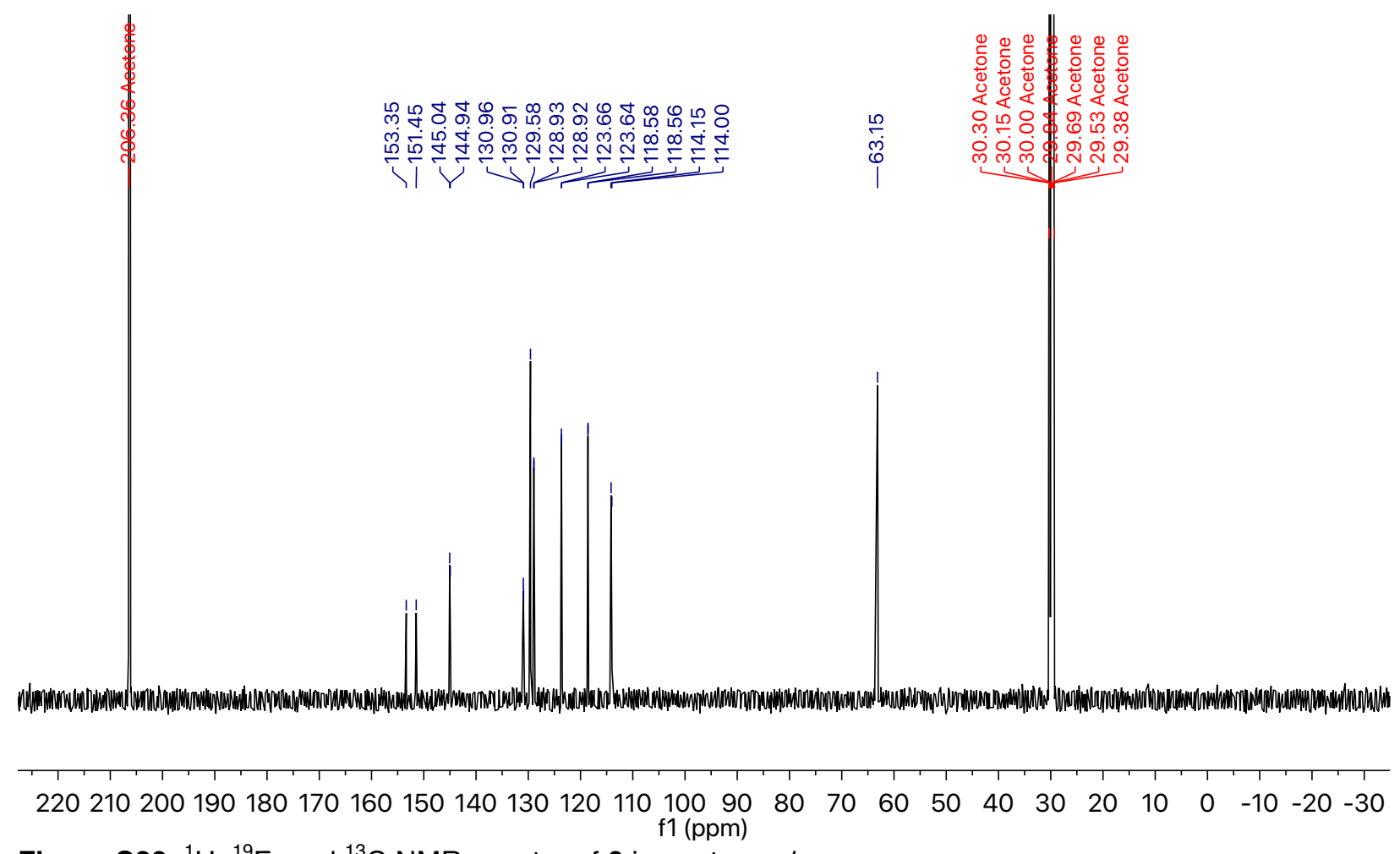

Figure S29. ${ }^{1} \mathrm{H},{ }^{19} \mathrm{~F}$, and ${ }^{13} \mathrm{C}$ NMR spectra of 6 in acetone- $d_{6}$ 


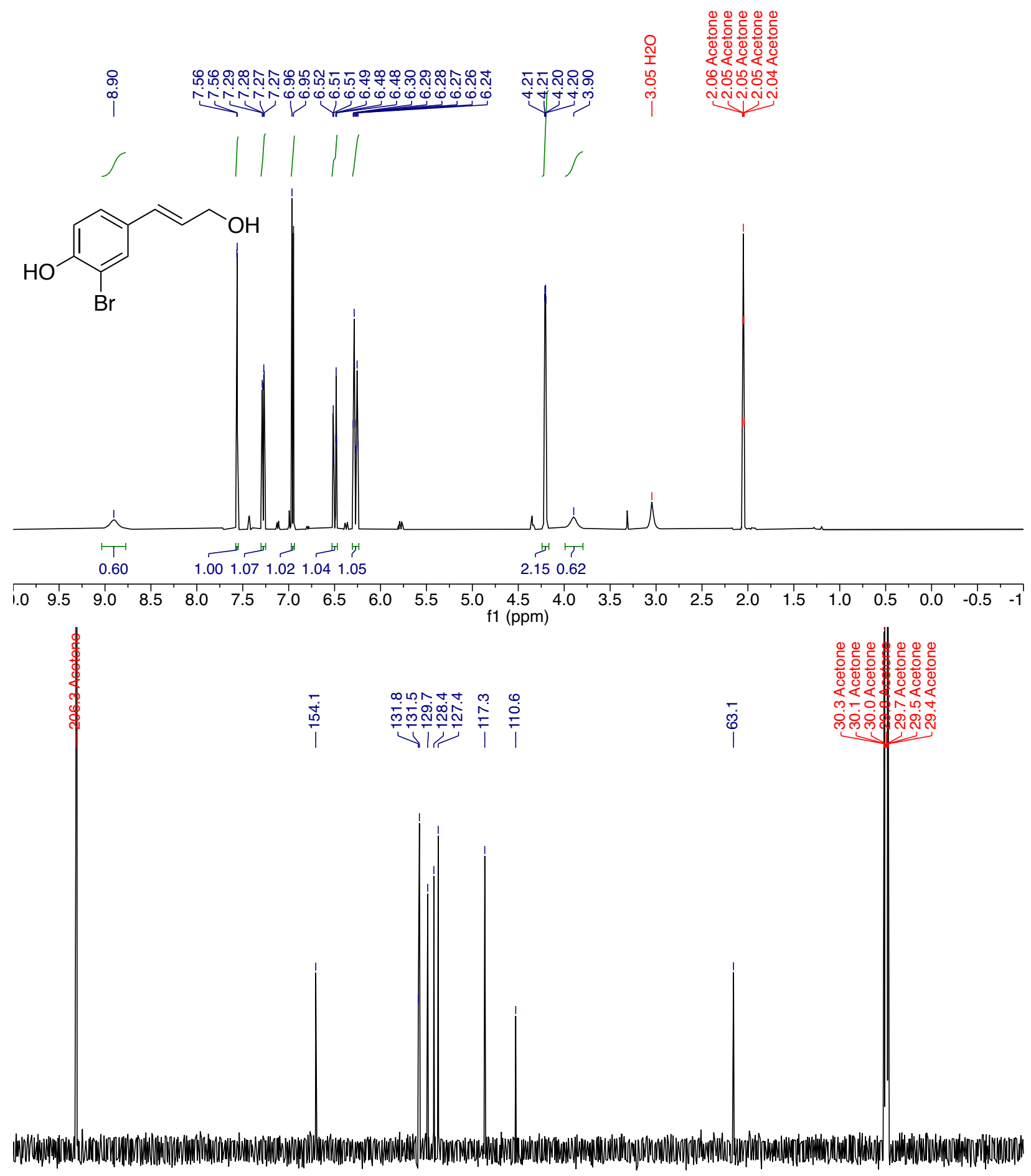

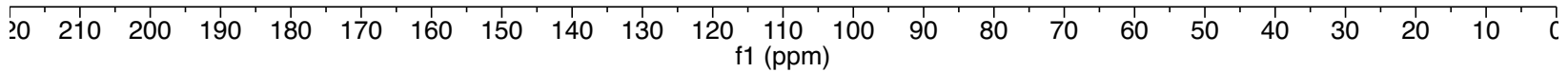

Figure S30. ${ }^{1} \mathrm{H}$ and ${ }^{13} \mathrm{C}$ NMR spectra of 7 in acetone- $d_{6}$ 

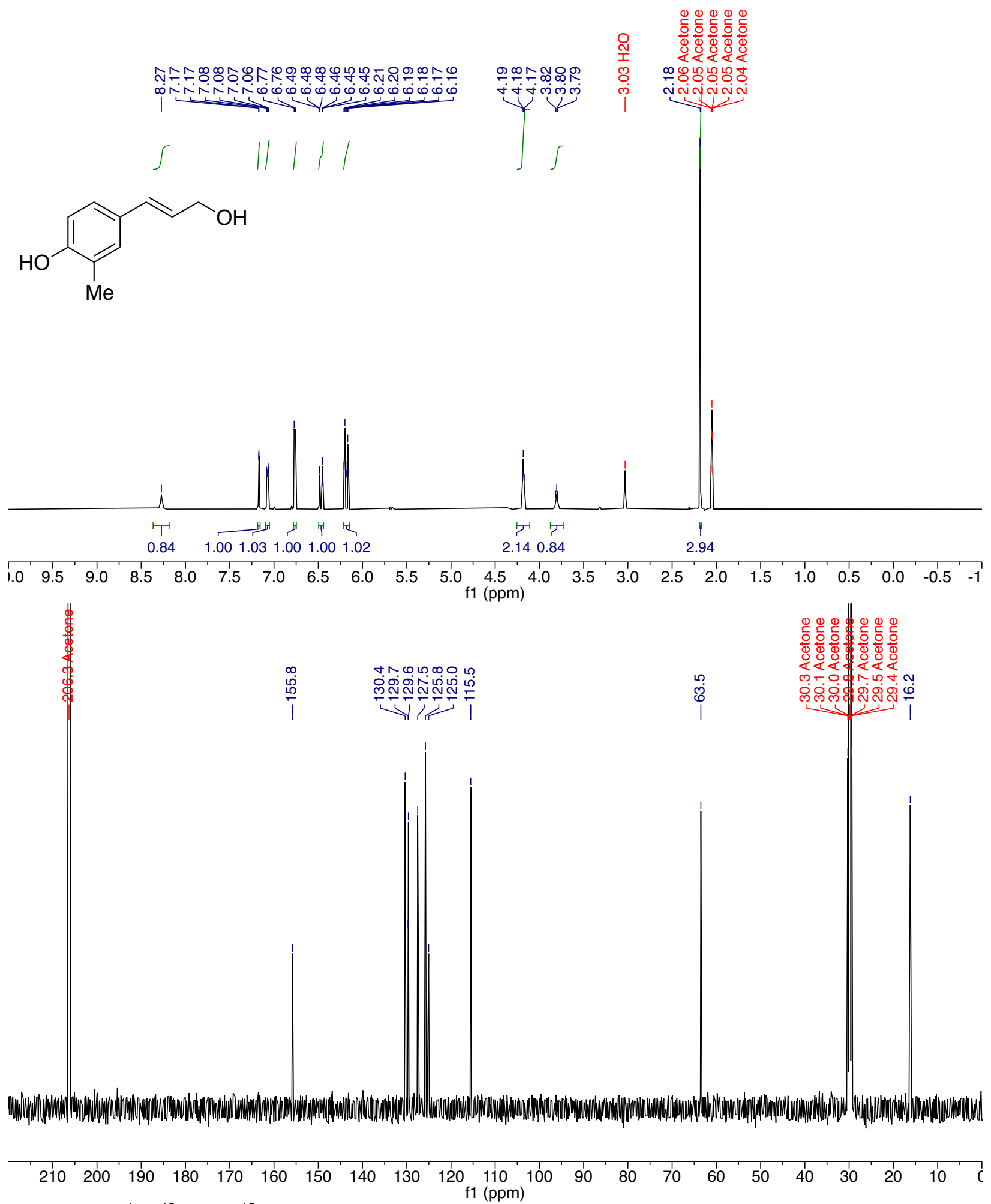

Figure S31. ${ }^{1} \mathrm{H},{ }^{19} \mathrm{~F}$, and ${ }^{13} \mathrm{C}$ NMR spectra of 8 in acetone- $d_{6}$ 


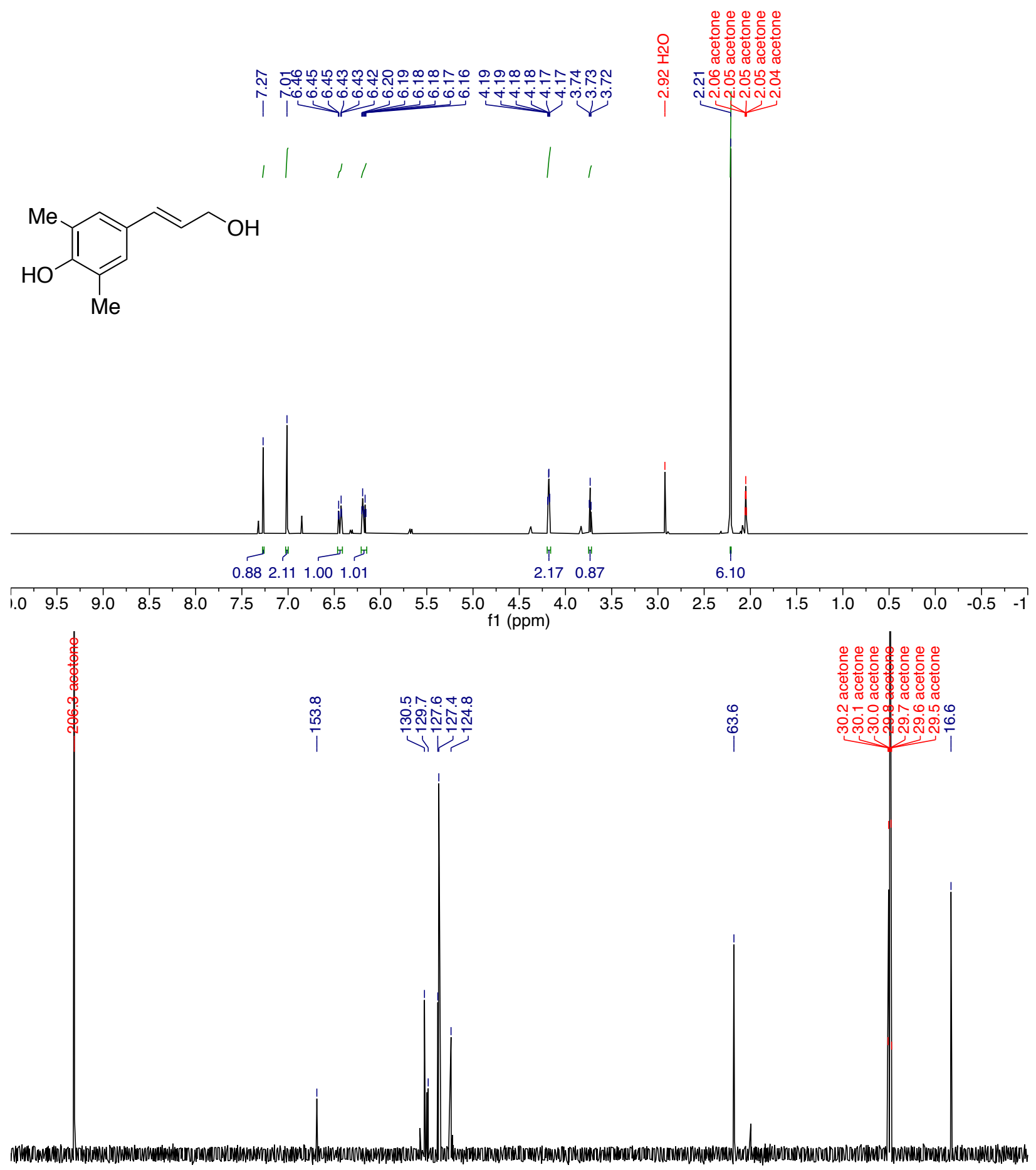

$\begin{array}{llllllllllllllllllllllllllllll}210 & 200 & 190 & 180 & 170 & 160 & 150 & 140 & 130 & 120 & 110 & 100 & 90 & 80 & 70 & 60 & 50 & 40 & 30 & 20 & 10 & \mathrm{C}\end{array}$

Figure S32. ${ }^{1} \mathrm{H},{ }^{19} \mathrm{~F}$, and ${ }^{13} \mathrm{C}$ NMR spectra of 9 in acetone- $d_{6}$ 


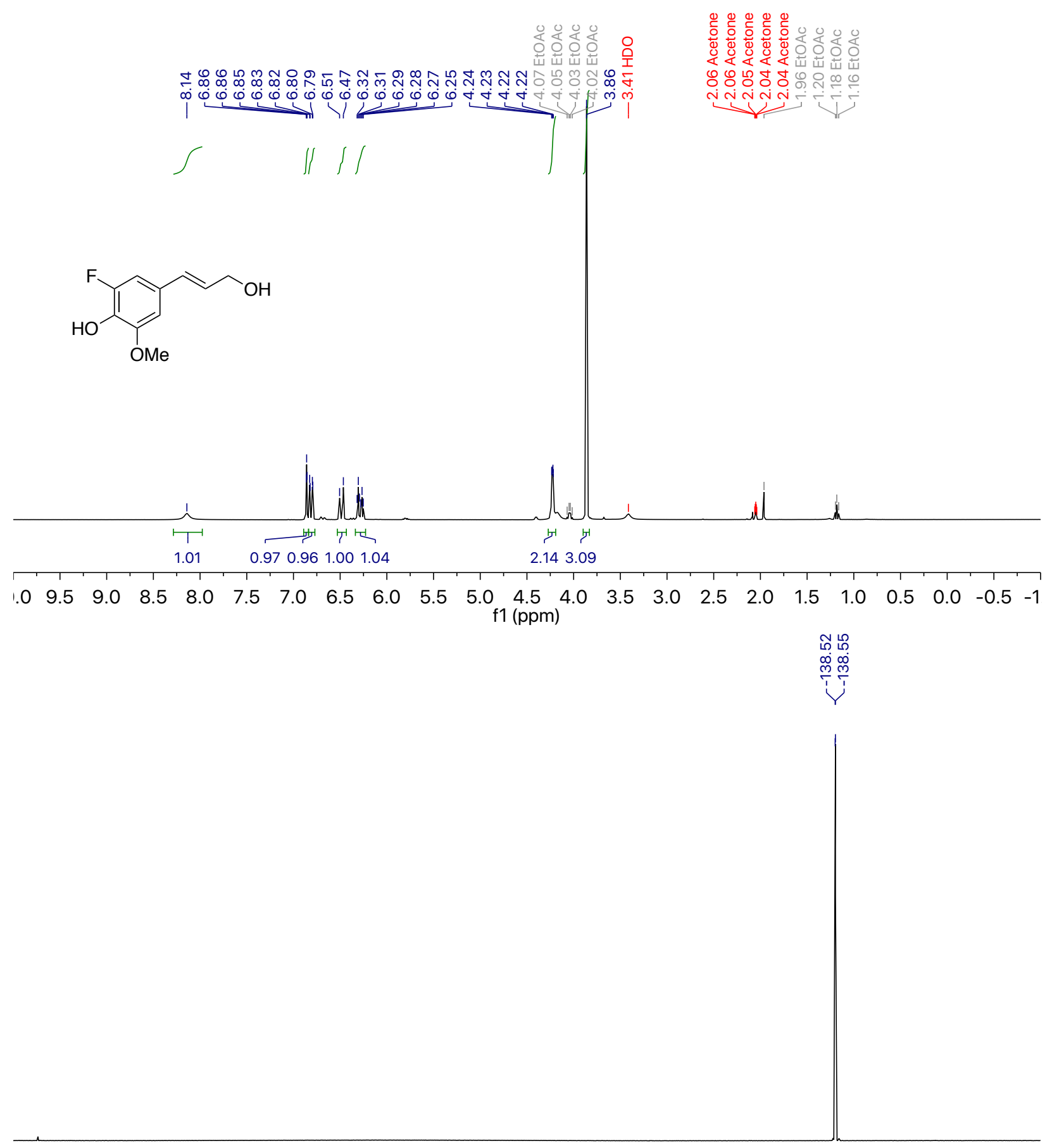

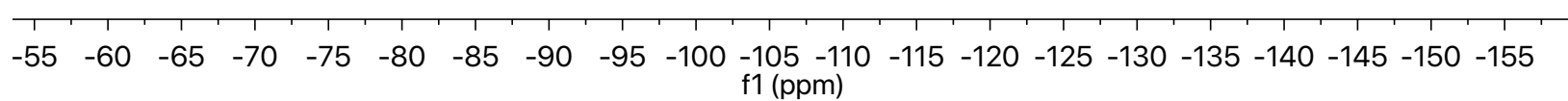




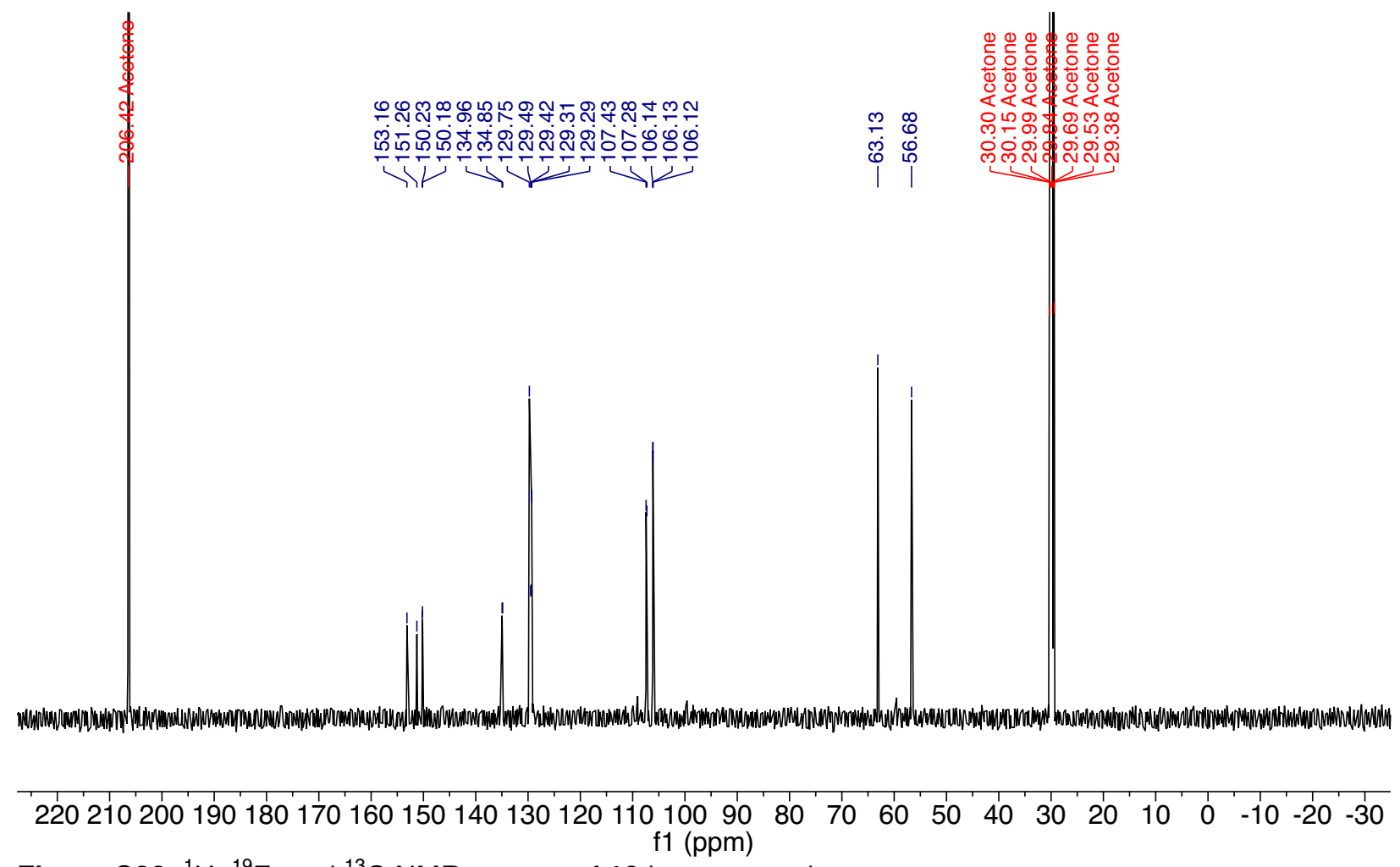

Figure S33. ${ }^{1} \mathrm{H},{ }^{19} \mathrm{~F}$, and ${ }^{13} \mathrm{C}$ NMR spectra of $\mathbf{1 0}$ in acetone- $d_{6}$ 

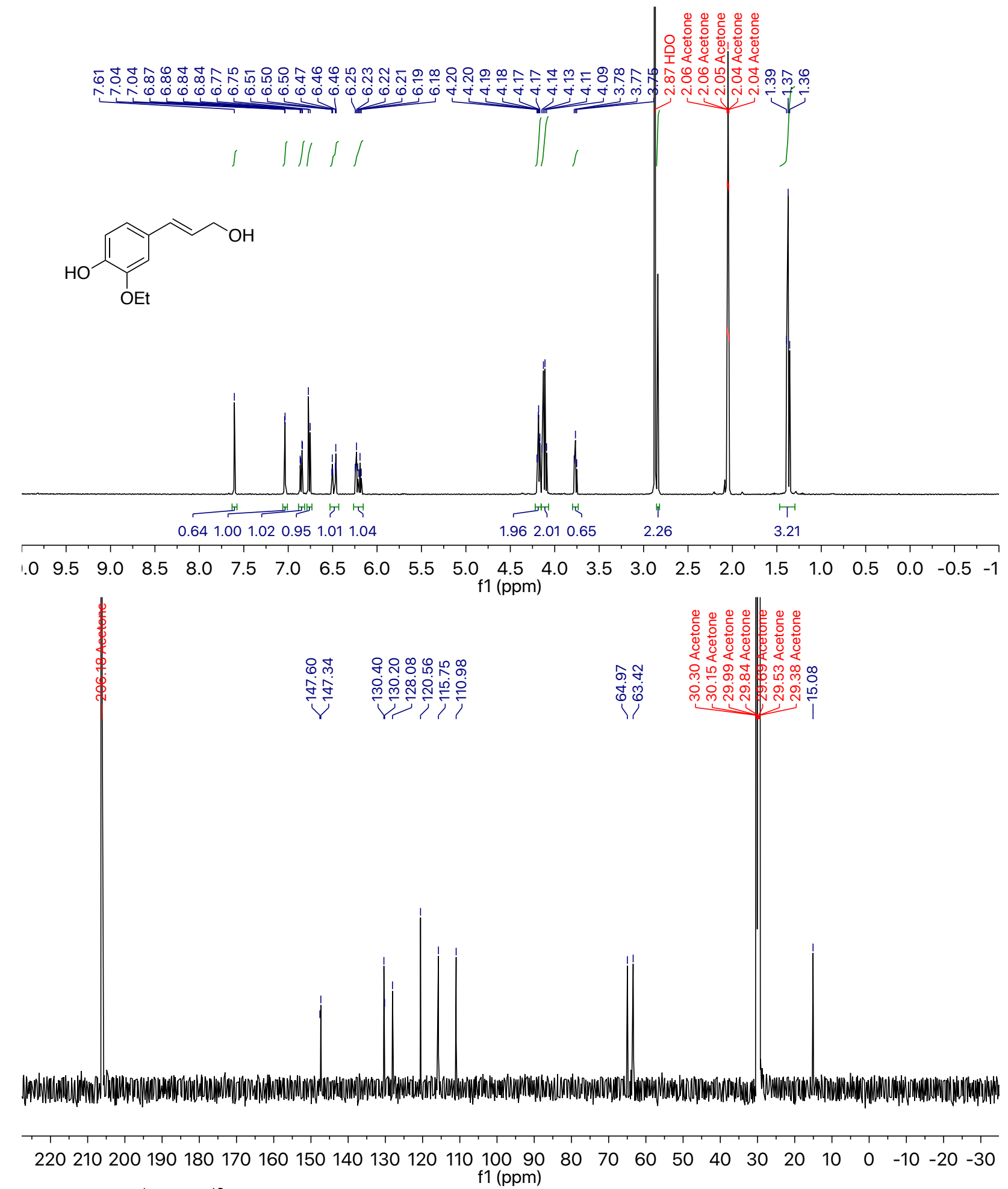

Figure S34. ${ }^{1} \mathrm{H}$ and ${ }^{13} \mathrm{C}$ NMR spectra of 11 in acetone- $d_{6}$ 

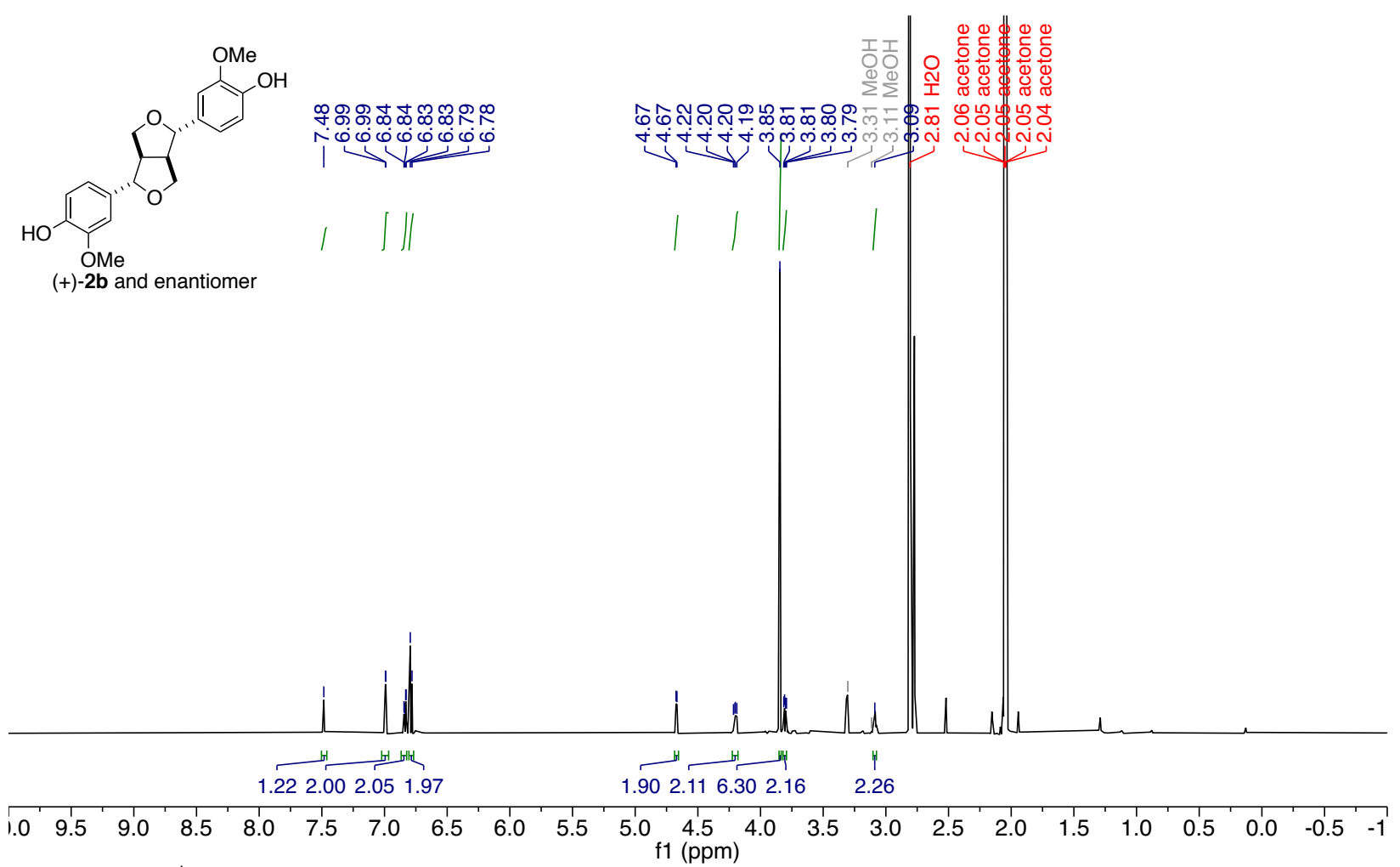

Figure S35. ${ }^{1} \mathrm{H}$ NMR spectrum of $\mathbf{2} \mathbf{b}$ in acetone- $d_{6}$ 


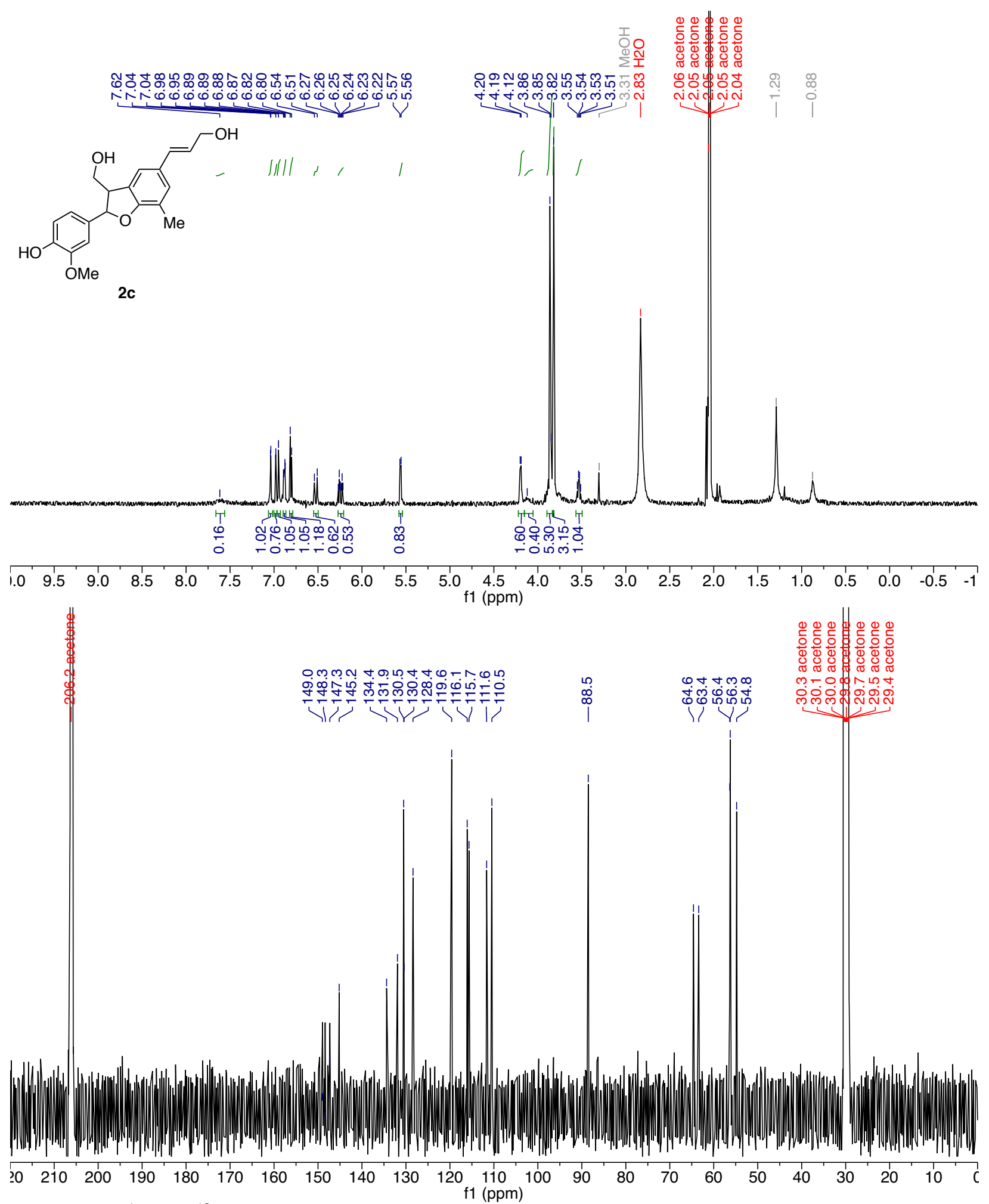

Figure S36. ${ }^{1} \mathrm{H}$ and ${ }^{13} \mathrm{C}$ NMR spectra of $2 \mathrm{c}$ in acetone- $d_{6}$ 


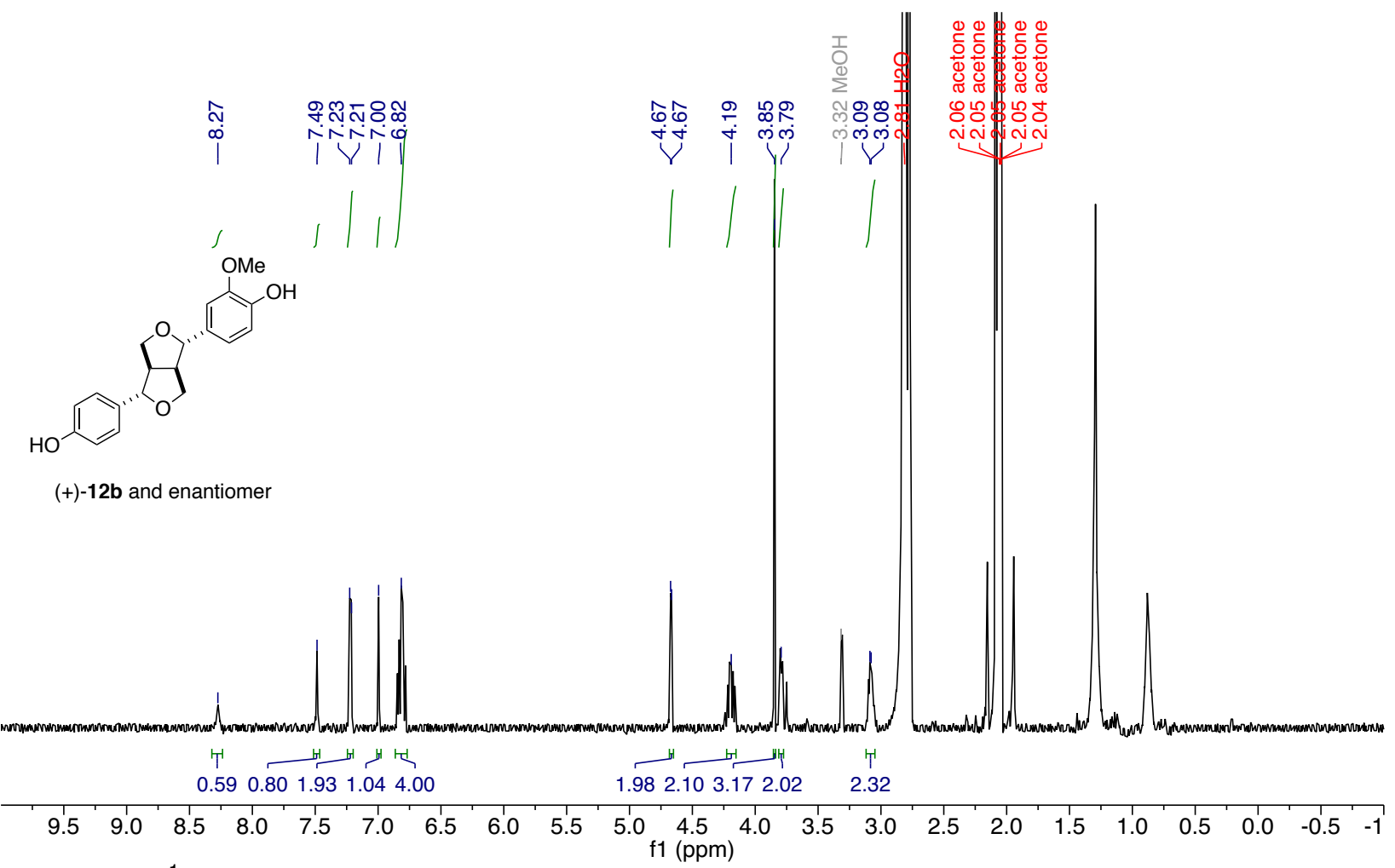

Figure S37. ${ }^{1} \mathrm{H}$ NMR spectrum of $12 \mathbf{b}$ in acetone- $d_{6}$ 

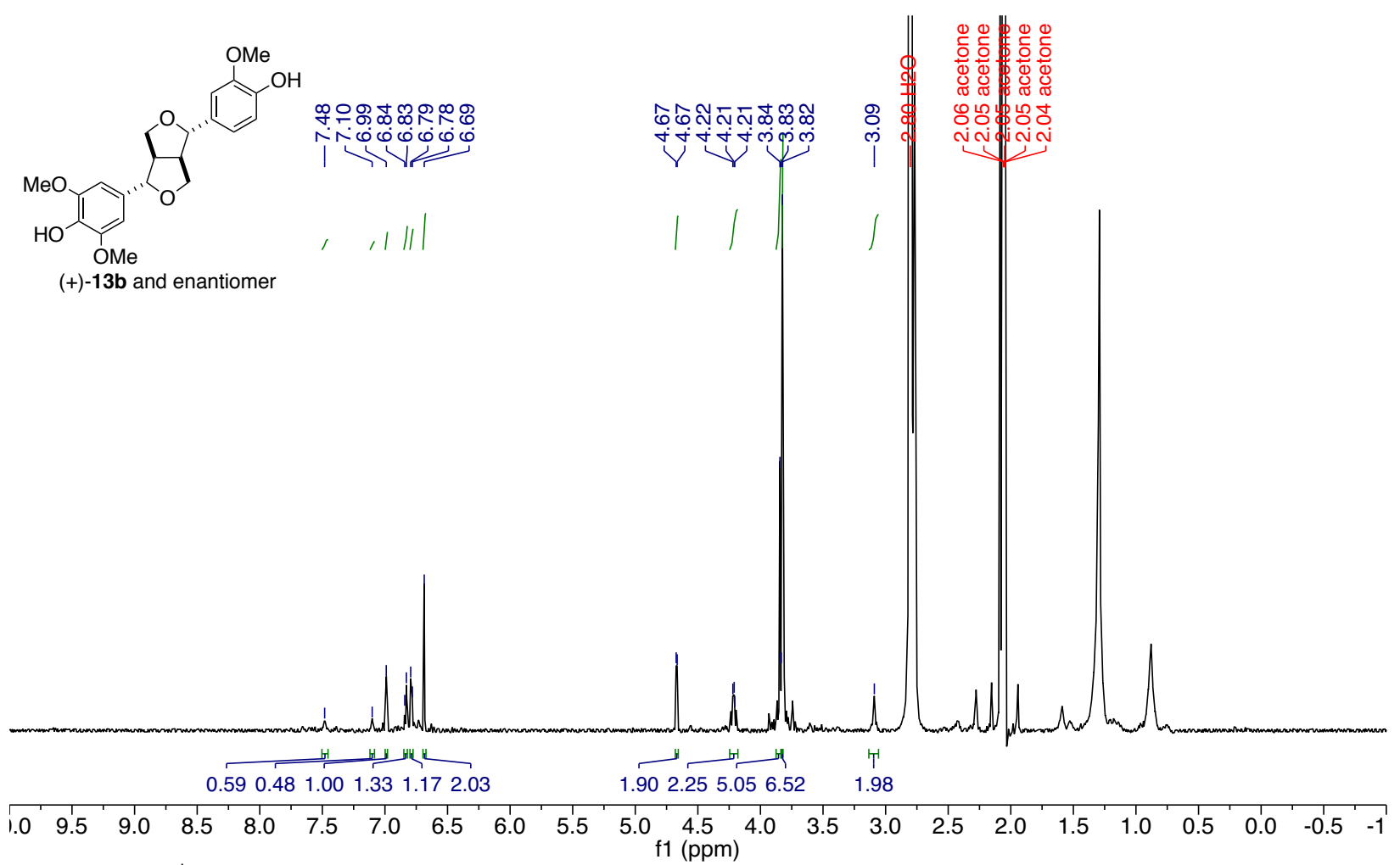

Figure S38. ${ }^{1} \mathrm{H}$ NMR spectrum of $\mathbf{1 3 b}$ in acetone- $d_{6}$ 

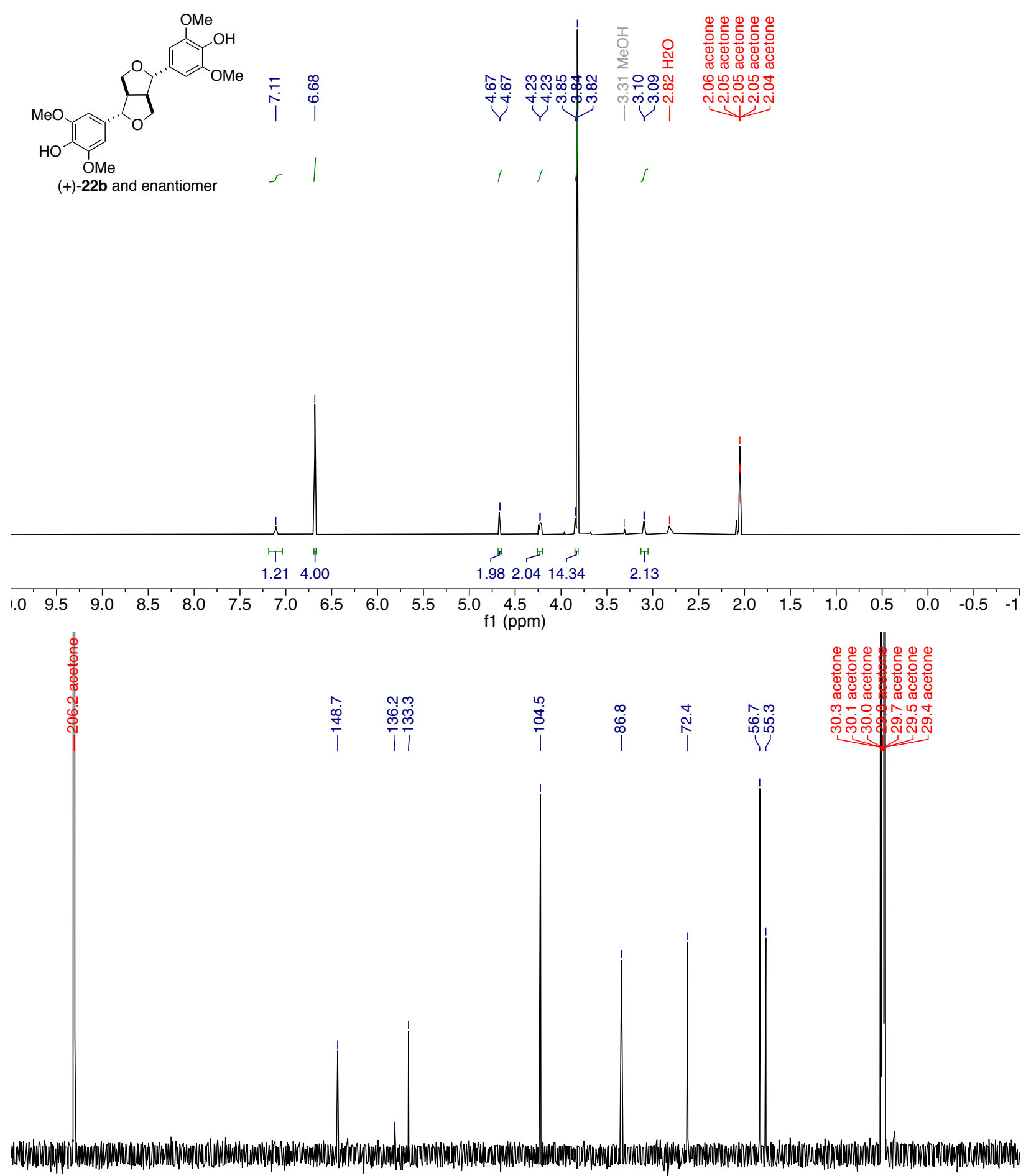

$\begin{array}{lllllllllllllllllllllll}20 & 210 & 200 & 190 & 180 & 170 & 160 & 150 & 140 & 130 & 120 & 110 & 100 & 90 & 80 & 70 & 60 & 50 & 40 & 30 & 20 & 10 & \mathrm{C}\end{array}$

Figure S39. ${ }^{1} \mathrm{H}$ and ${ }^{13} \mathrm{C}$ NMR spectra of $22 \mathrm{~b}$ in acetone- $d_{6}$ 

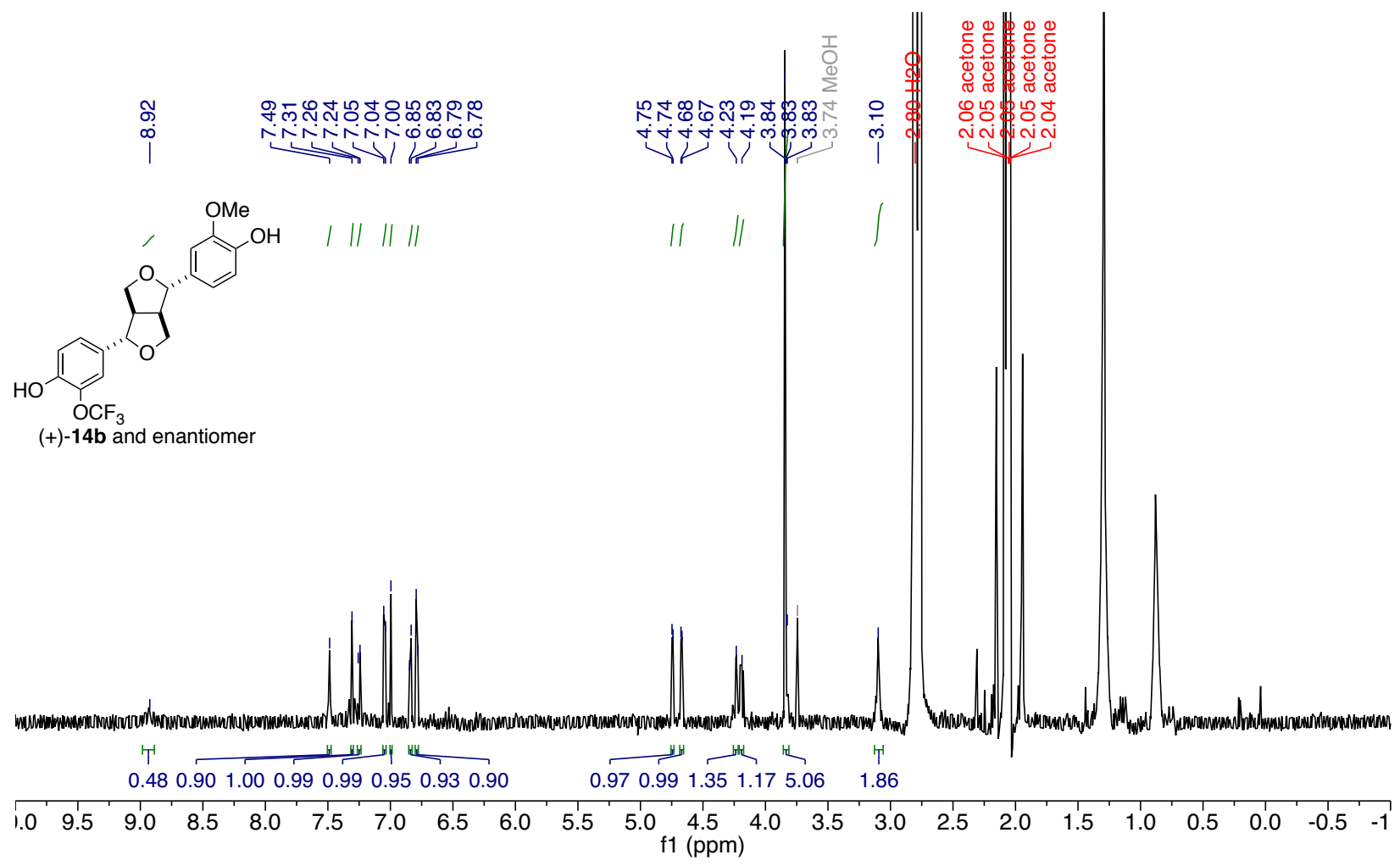

م

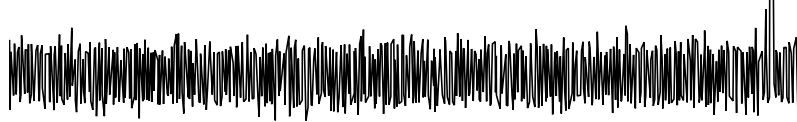

10 $-45$ $-50$ $-55$ $\mathrm{f1}(\mathrm{ppm})$ $-65$

Figure S40. ${ }^{1} \mathrm{H}$ and ${ }^{19} \mathrm{~F}$ NMR spectra of $14 \mathrm{~b}$ in acetone- $d_{6}$ 

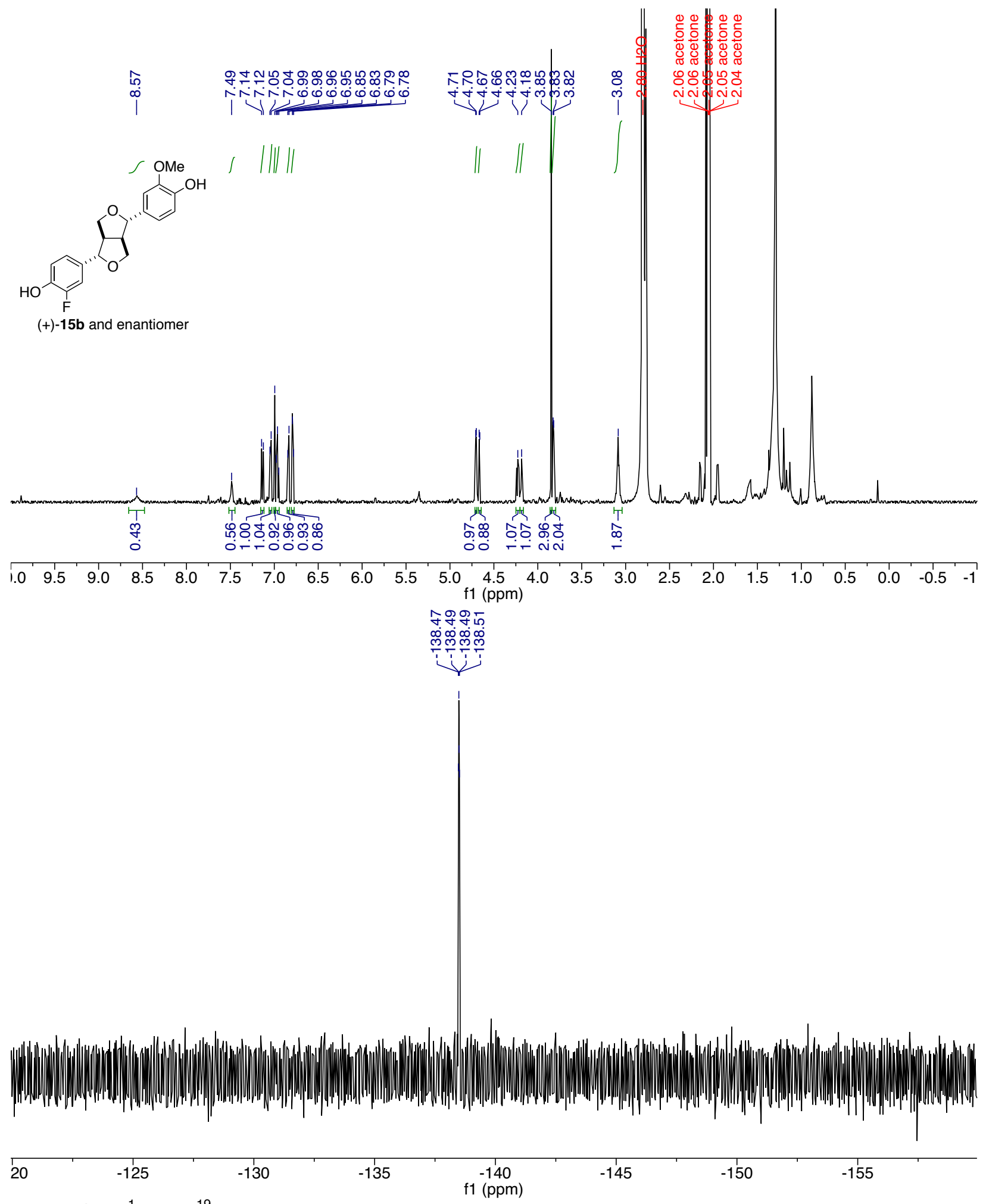

Figure S41. ${ }^{1} \mathrm{H}$ and ${ }^{19} \mathrm{~F}$ NMR spectra of $15 \mathrm{~b}$ in acetone- $d_{6}$ 

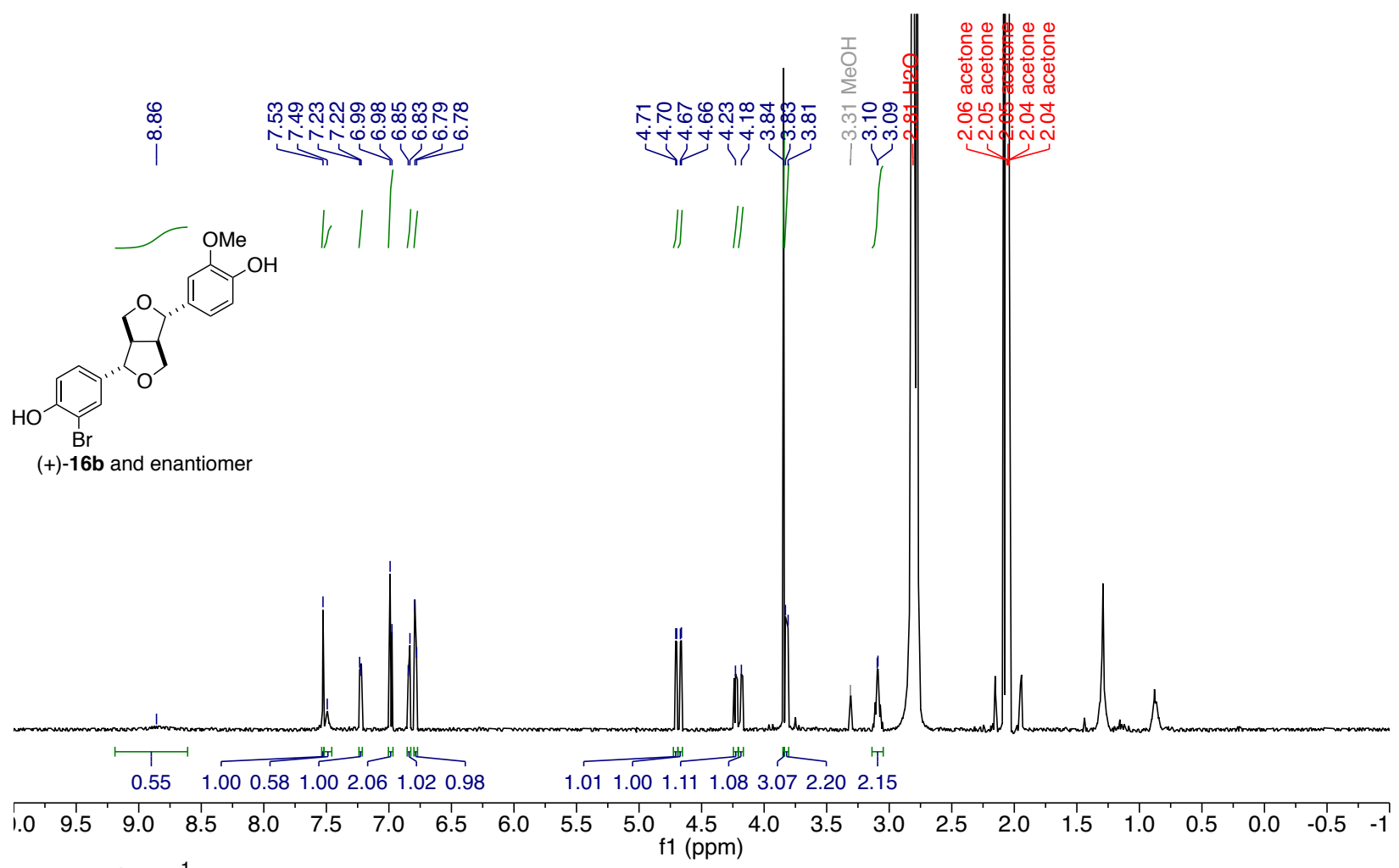

Figure S42. ${ }^{1} \mathrm{H}$ NMR spectrum of $16 \mathbf{b}$ in acetone- $d_{6}$ 


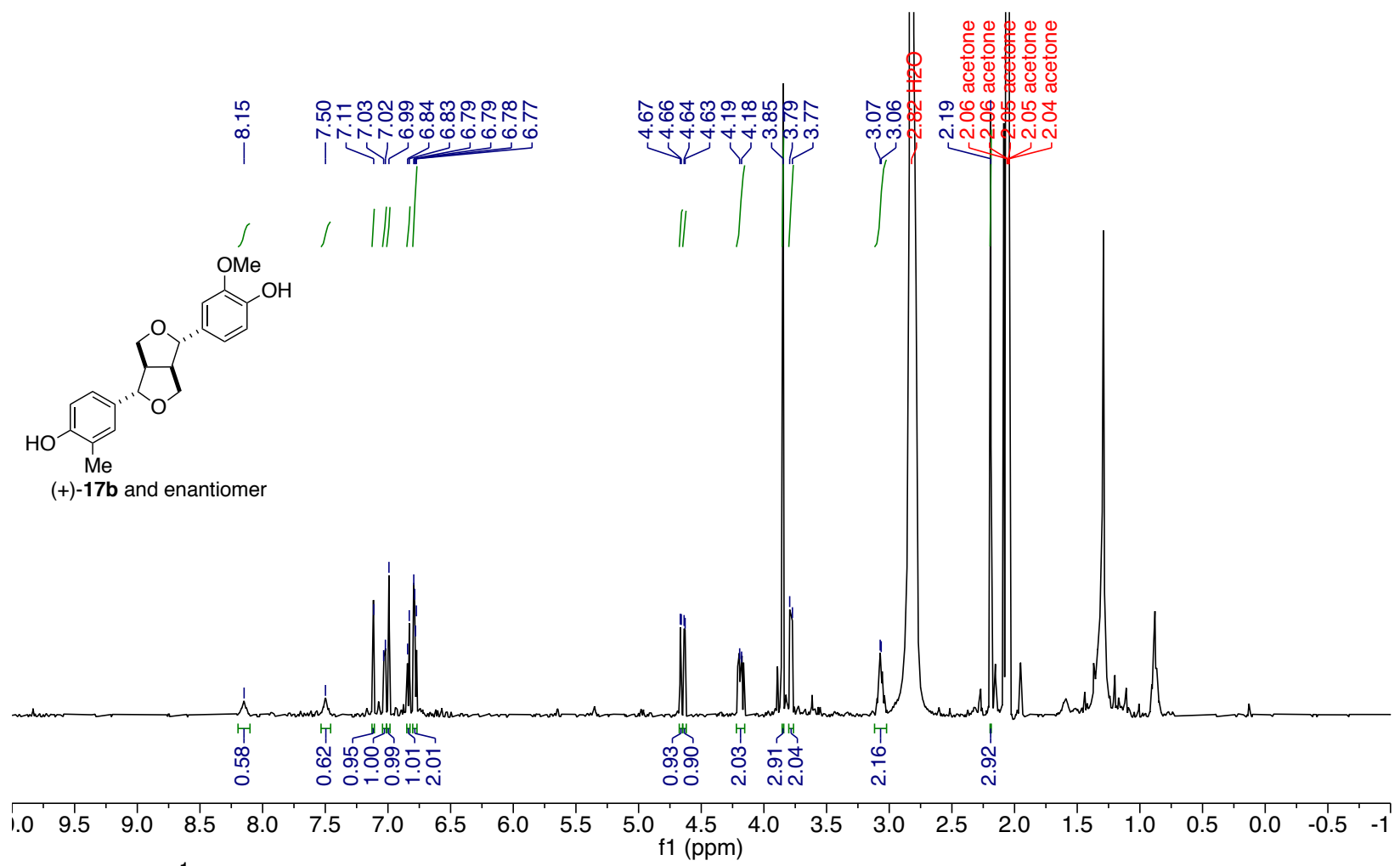

Figure S43. ${ }^{1} \mathrm{H}$ NMR spectrum of $17 \mathrm{~b}$ in acetone- $d_{6}$ 


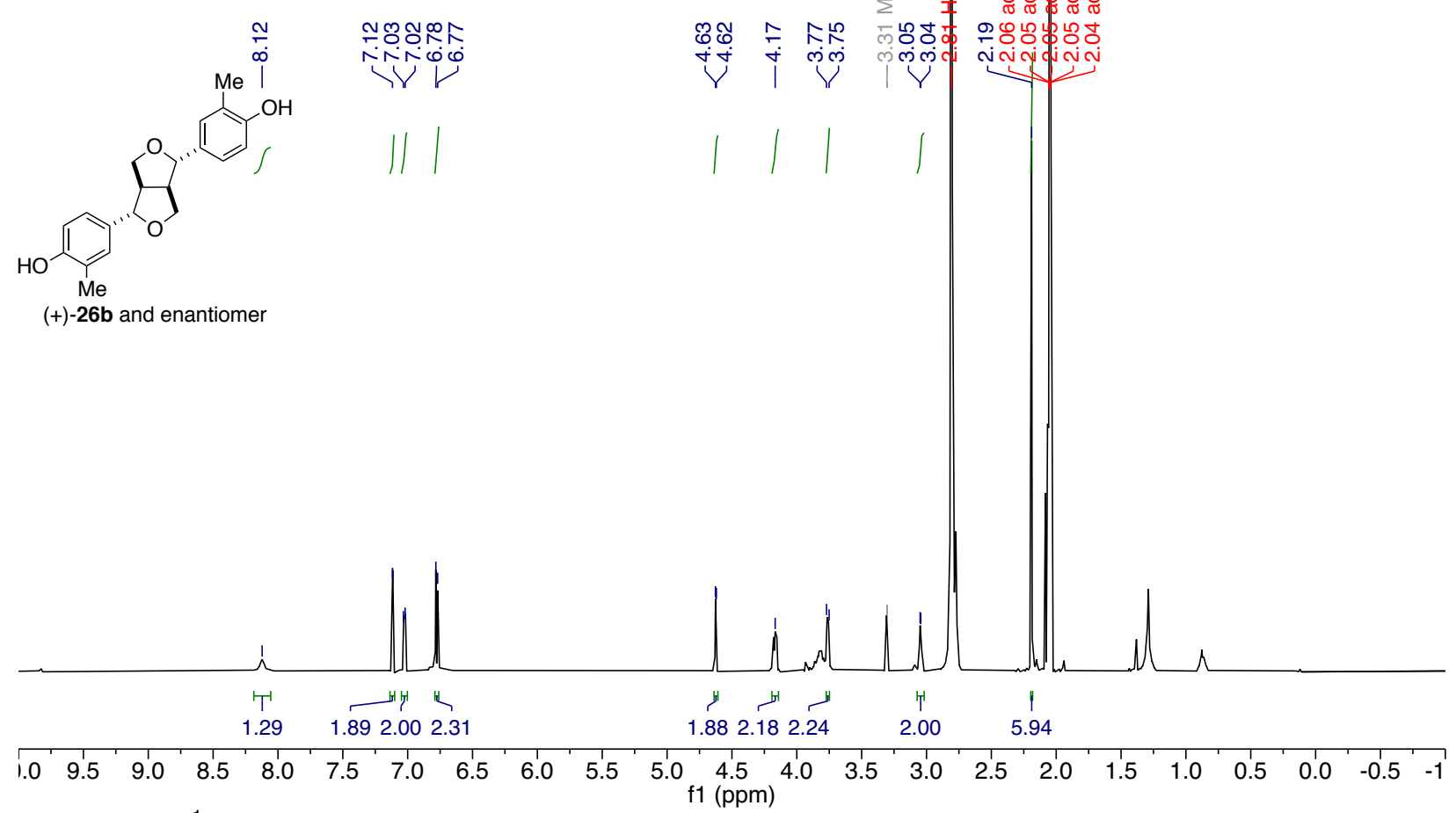

Figure S44. ${ }^{1} \mathrm{H}$ NMR spectrum of $\mathbf{2 6 b}$ in acetone- $d_{6}$ 

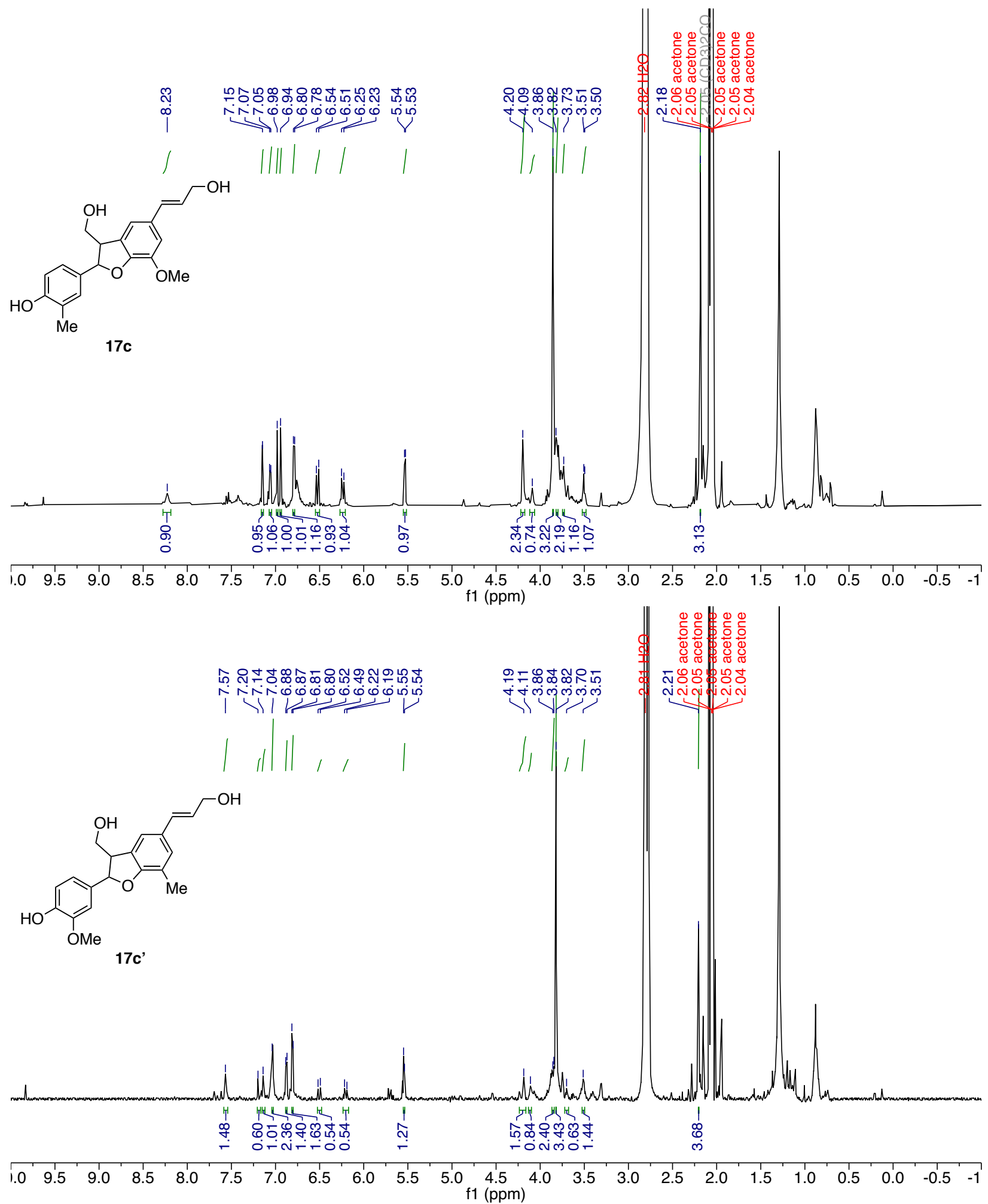

Figure S45. 'H NMR spectra of $17 \mathrm{c}$ and $17 \mathrm{c}$ ' in acetone- $d_{6}$ 


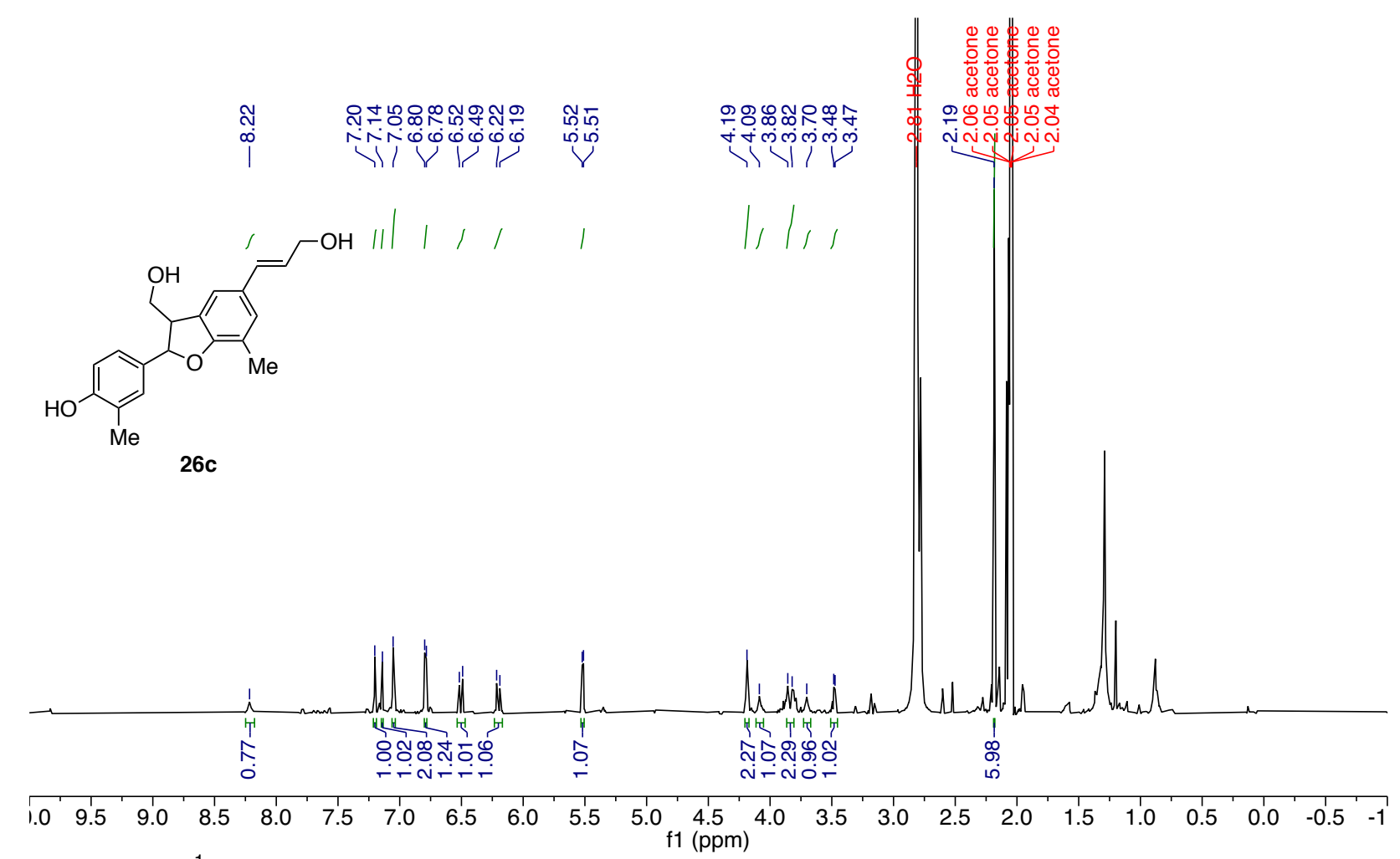

Figure S46. ${ }^{1} \mathrm{H}$ NMR spectrum of $26 \mathrm{c}$ in acetone- $d_{6}$ 

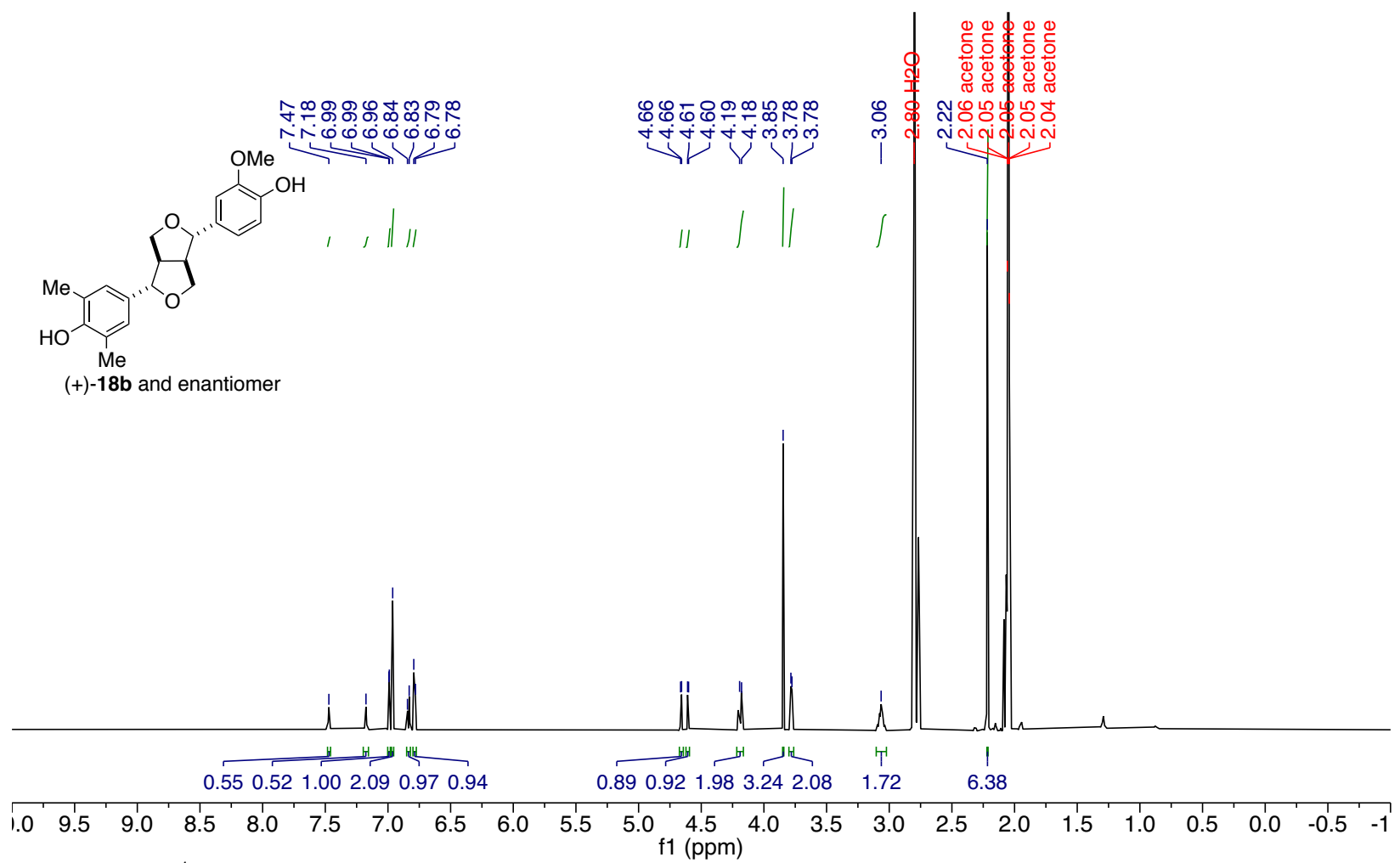

Figure S47. ${ }^{1} \mathrm{H}$ NMR spectrum of $18 \mathrm{~b}$ in acetone- $d_{6}$ 

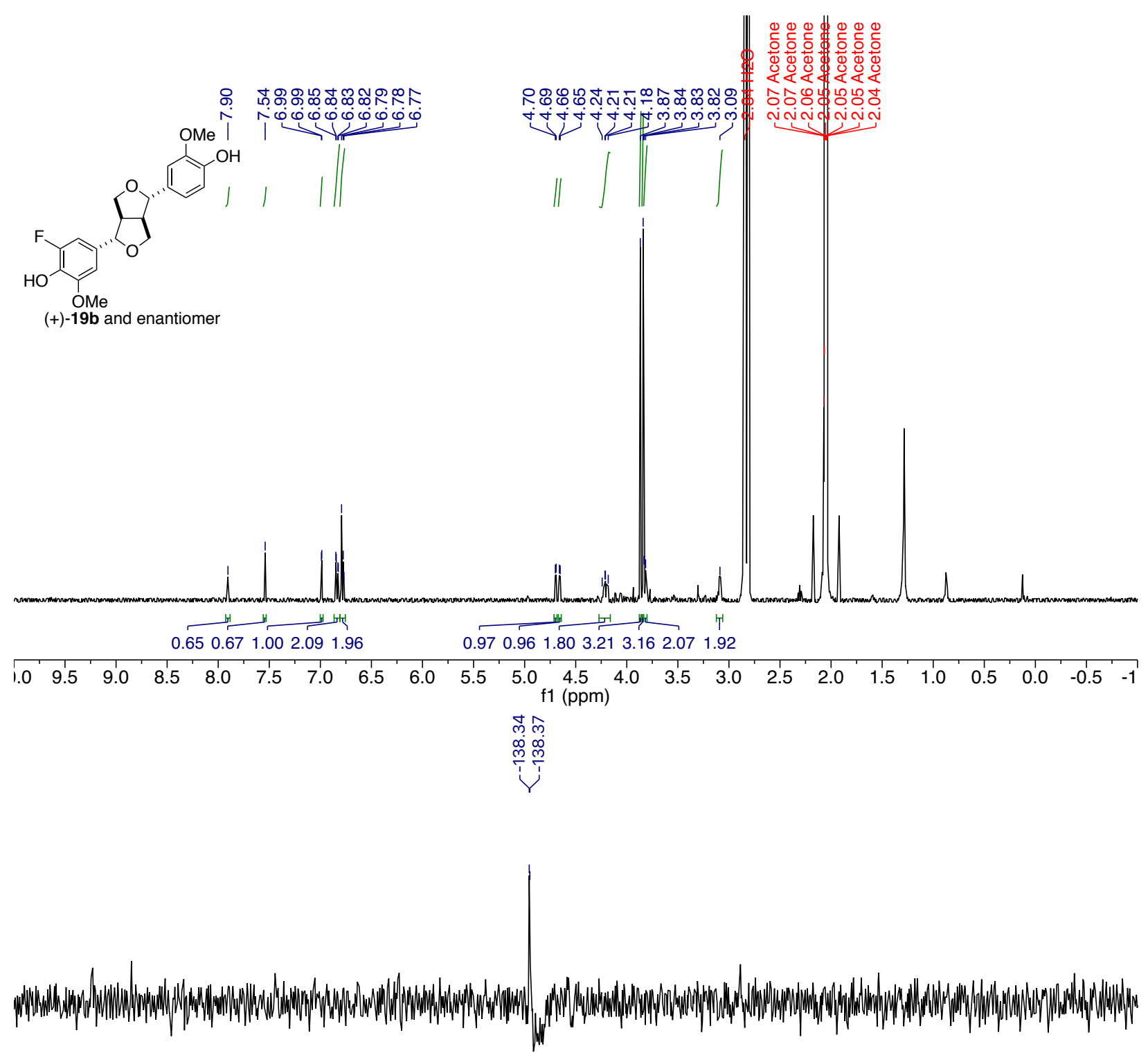

\begin{tabular}{|c|c|c|c|c|c|c|c|}
\hline 20 & -125 & -130 & -135 & $\begin{array}{c}-140 \\
\mathrm{f} 1(\mathrm{ppm})\end{array}$ & -145 & -150 & -155 \\
\hline
\end{tabular}

Figure S48. ${ }^{1} \mathrm{H}$ and ${ }^{19} \mathrm{~F}$ NMR spectra of $19 \mathrm{~b}$ in acetone- $d_{6}$ 

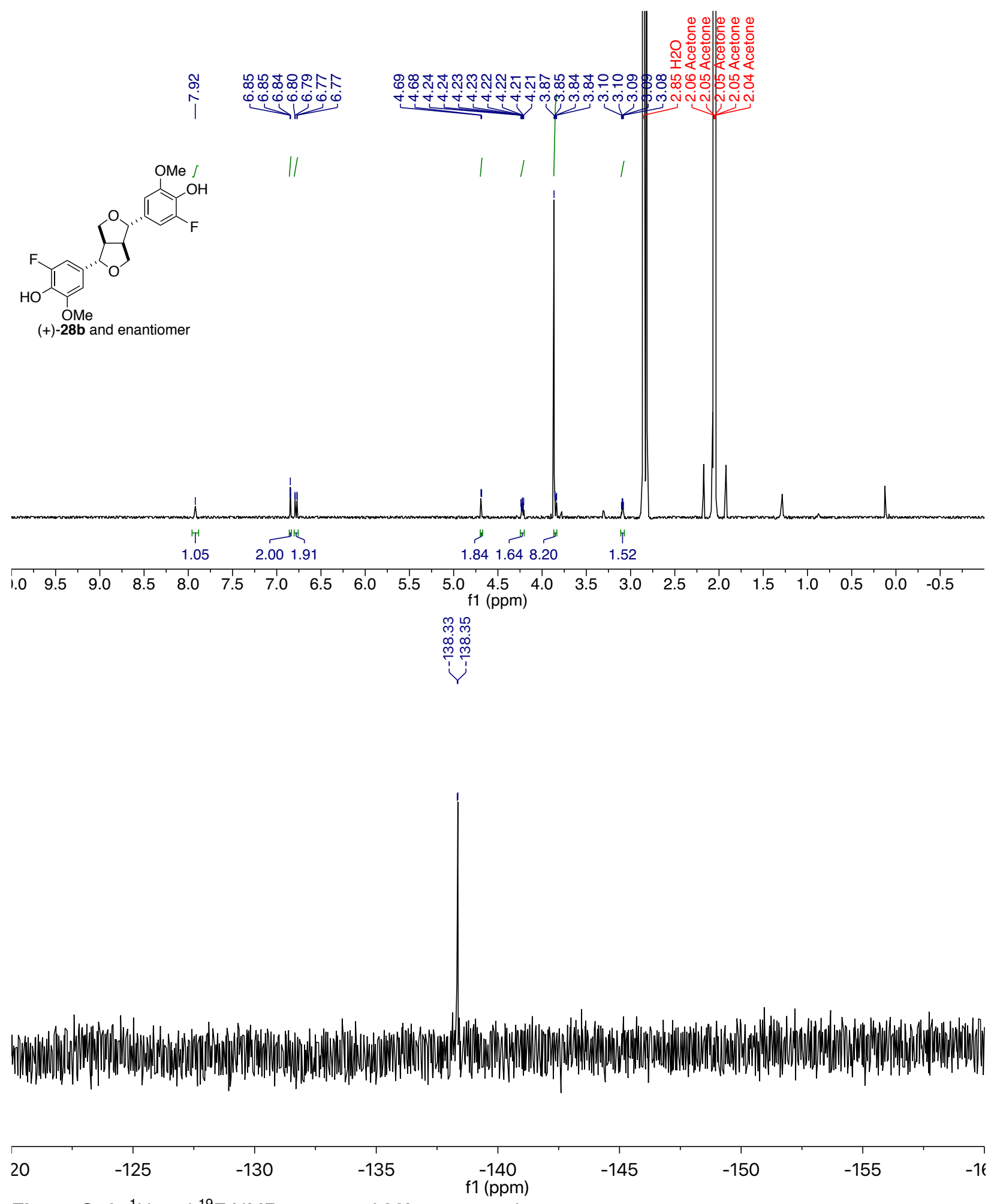

Figure S49. ${ }^{1} \mathrm{H}$ and ${ }^{19} \mathrm{~F}$ NMR spectra of $\mathbf{2 8} \mathbf{b}$ acetone- $d_{6}$ 


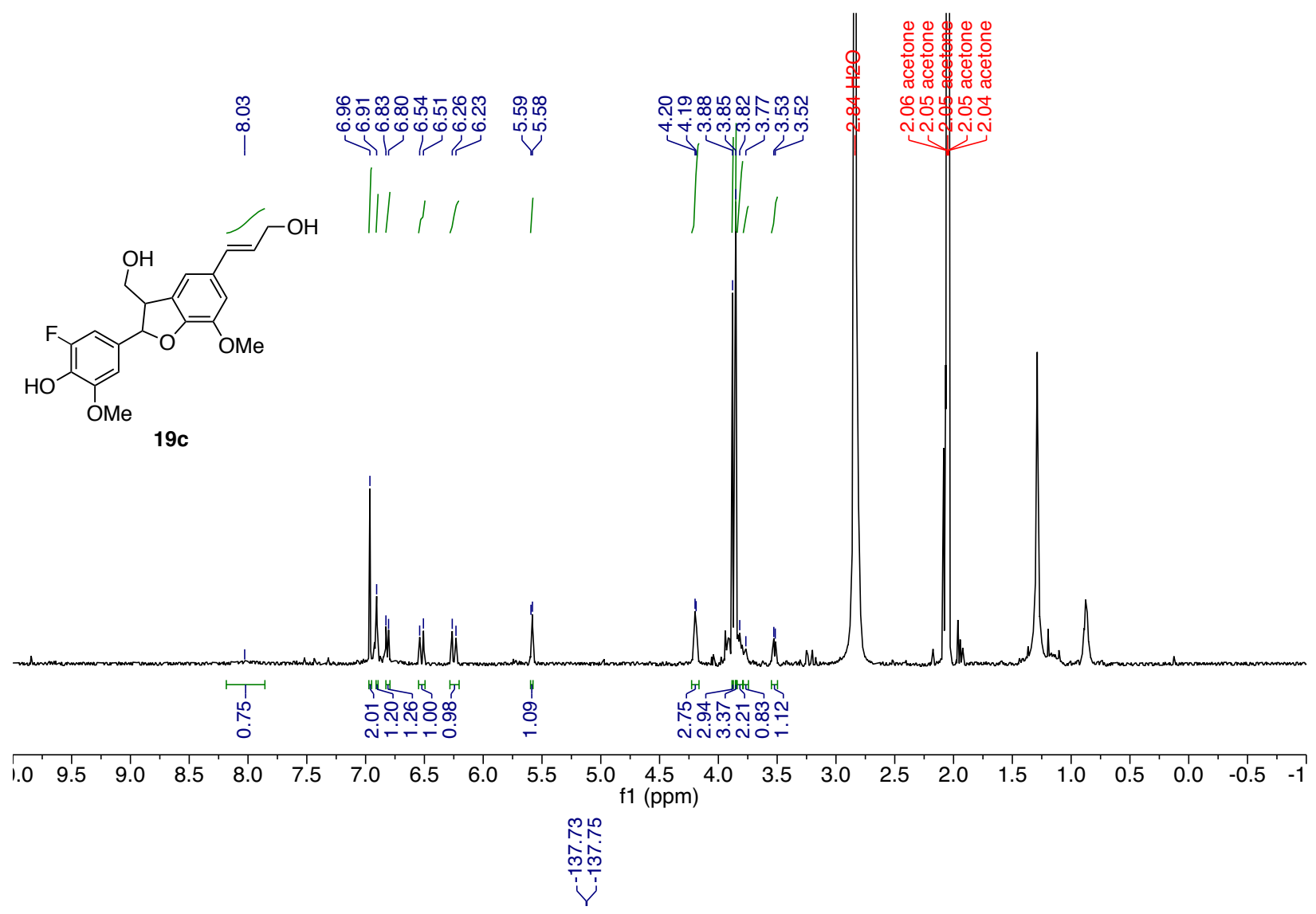

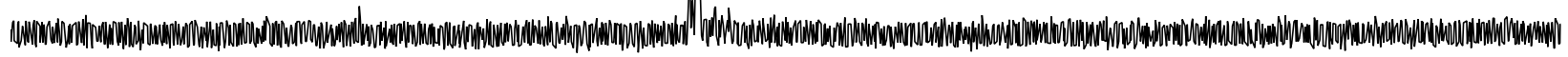

20

$-125$

$-130$

$-135$

f1 $\stackrel{-140}{(p p m)}$

$-145$

$-150$

$-155$

$-16$ 


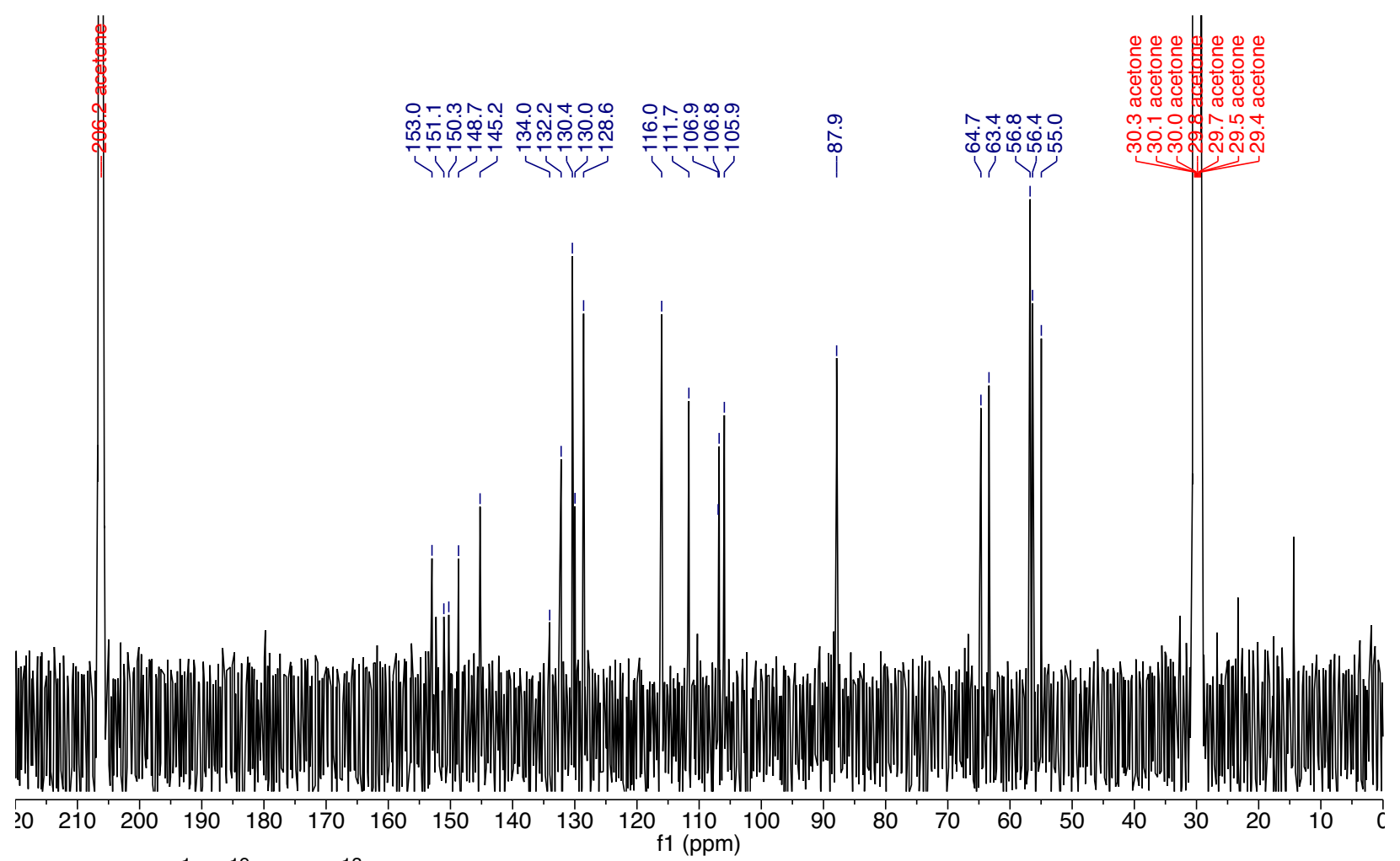

Figure S50. ${ }^{1} \mathrm{H},{ }^{19} \mathrm{~F}$, and ${ }^{13} \mathrm{C}$ NMR spectra of $19 \mathrm{c}$ acetone- $d_{6}$ 


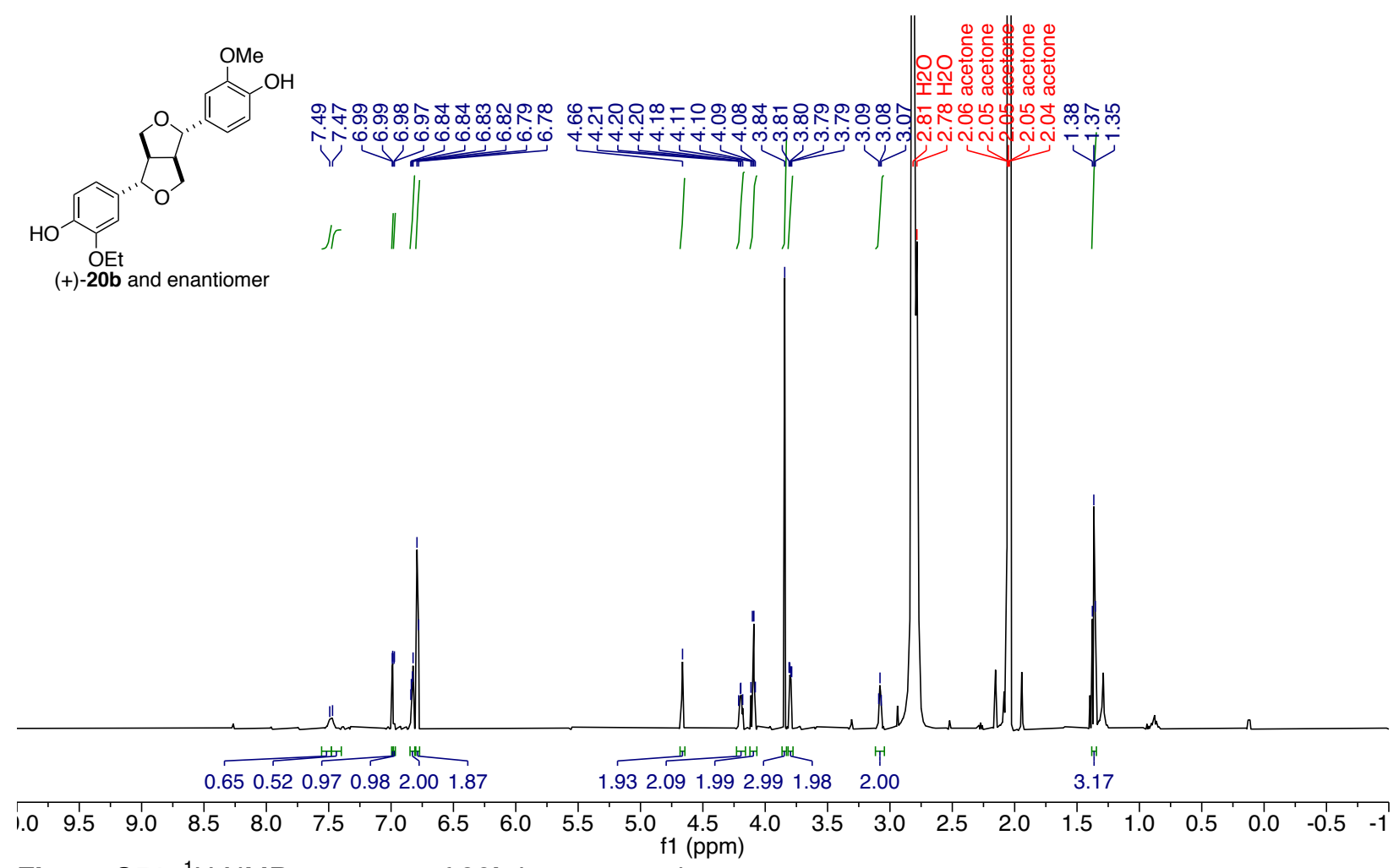

Figure S51. ${ }^{1} \mathrm{H}$ NMR spectrum of $20 \mathrm{~b}$ in acetone- $d_{6}$ 


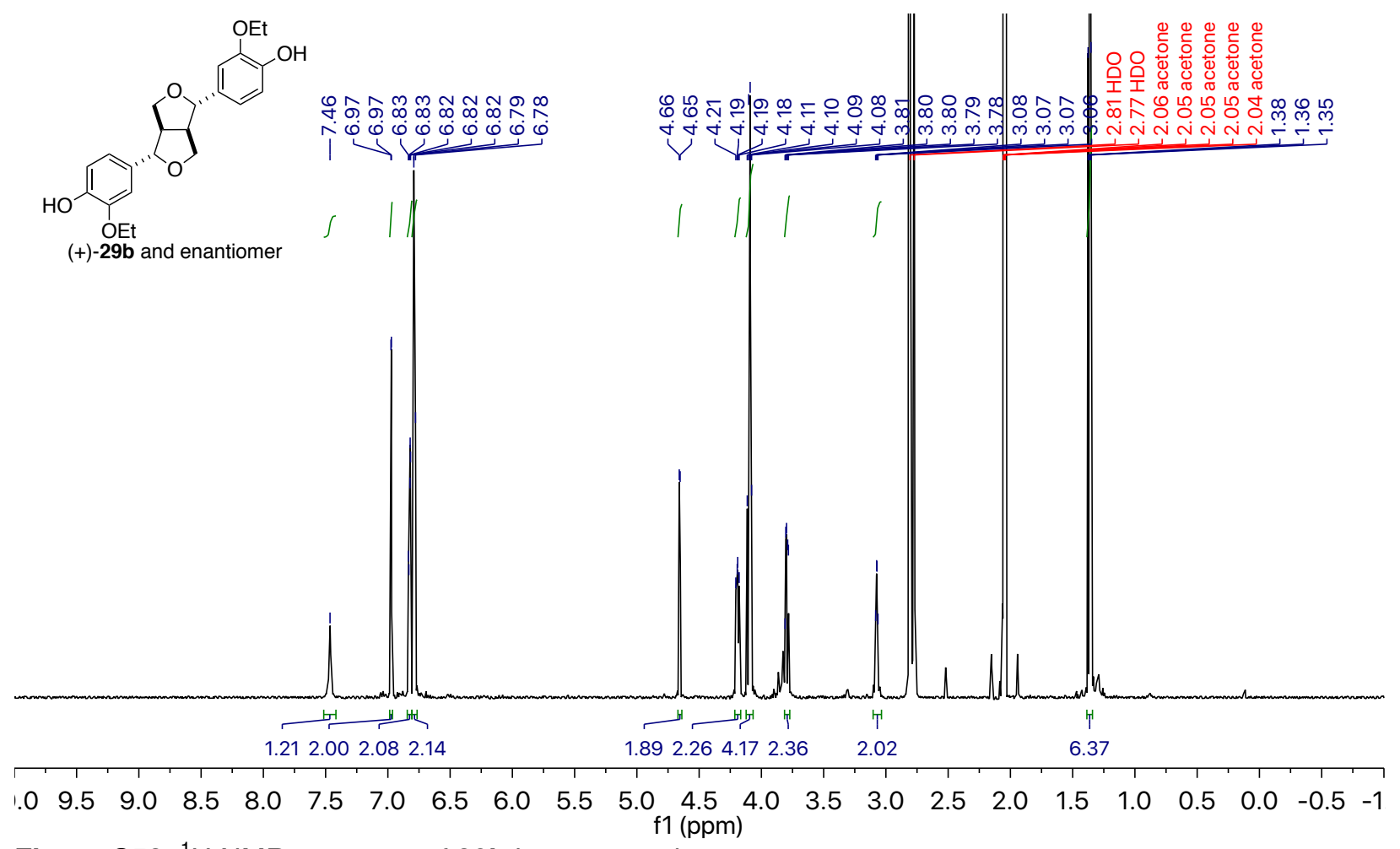

Figure S52. ${ }^{1} \mathrm{H}$ NMR spectrum of $29 \mathrm{~b}$ in acetone- $d_{6}$ 

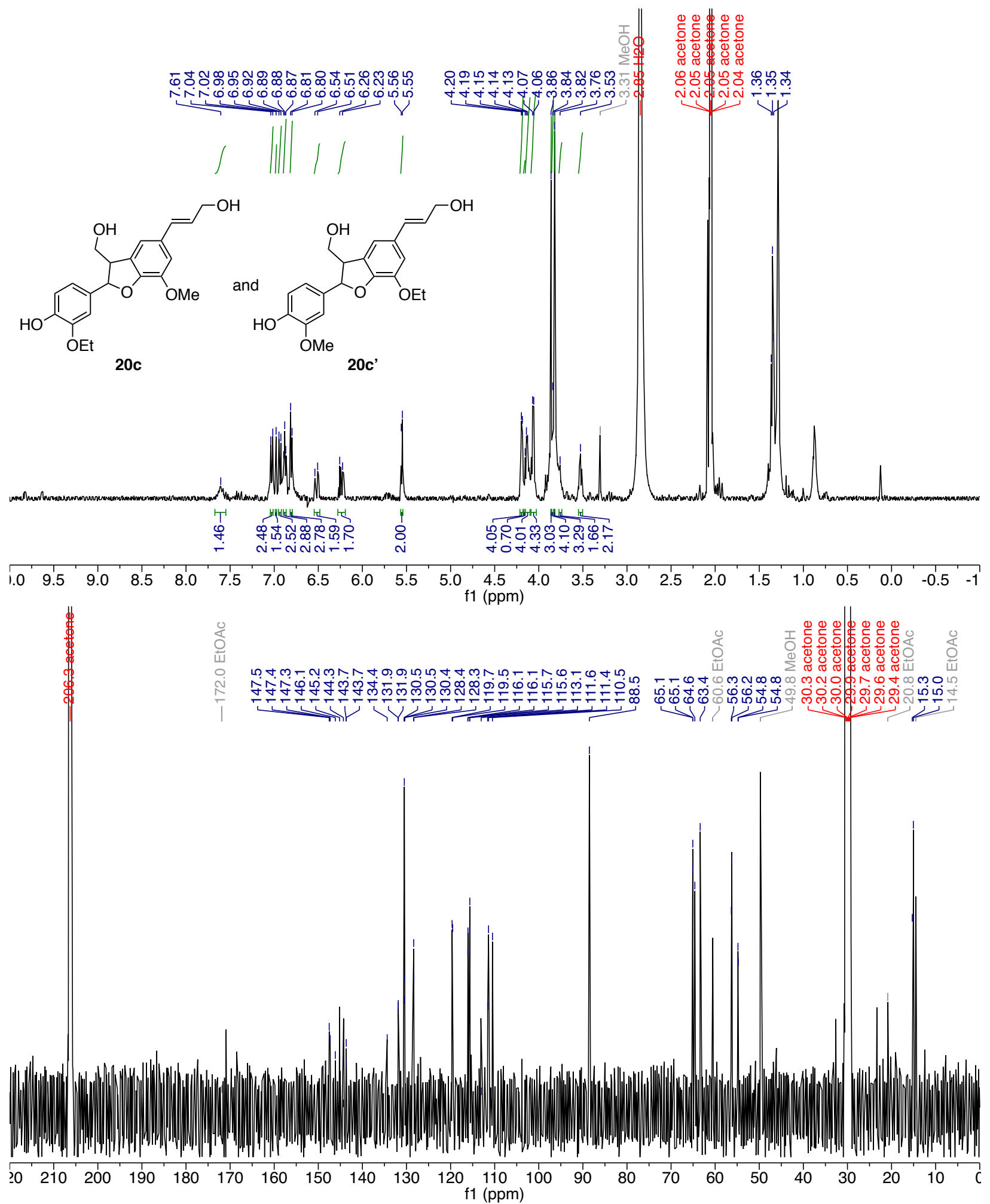

Figure S53. ${ }^{1} \mathrm{H}$ and ${ }^{13} \mathrm{C}$ NMR spectra of $20 \mathrm{c} / \mathbf{2 0 c}$ ' in acetone- $d_{6}$ 

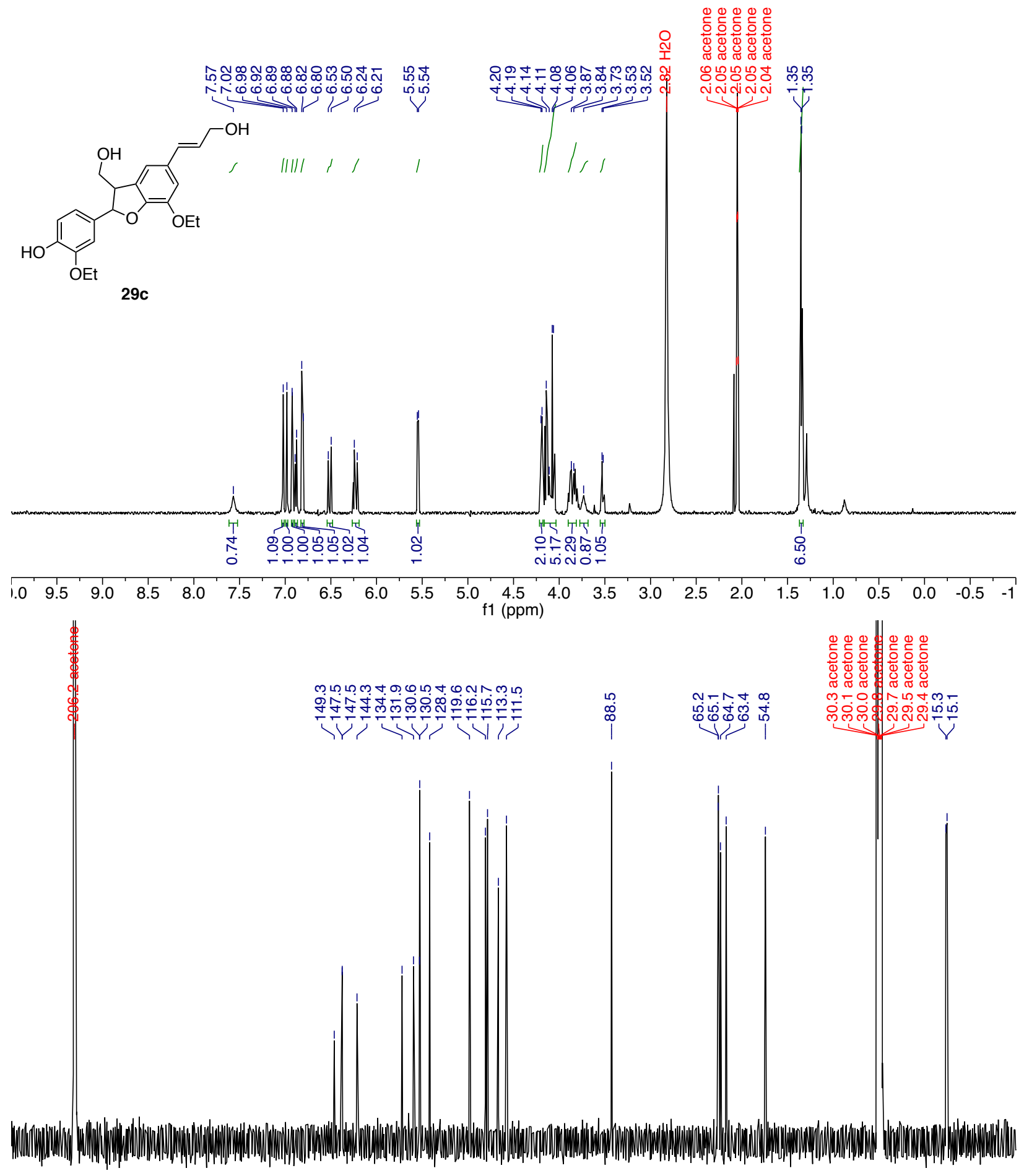

$\begin{array}{llllllllllllllllllllll}20 & 210 & 200 & 190 & 180 & 170 & 160 & 150 & 140 & 130 & 120 & \begin{array}{c}110 \\ \mathrm{f} 1 \\ (\mathrm{ppm})\end{array} & 100 & 90 & 80 & 70 & 60 & 50 & 40 & 30 & 20 & 10\end{array}$

Figure S54. ${ }^{1} \mathrm{H}$ and ${ }^{13} \mathrm{C}$ NMR spectra of $29 \mathrm{c}$ in acetone- $d_{6}$ 


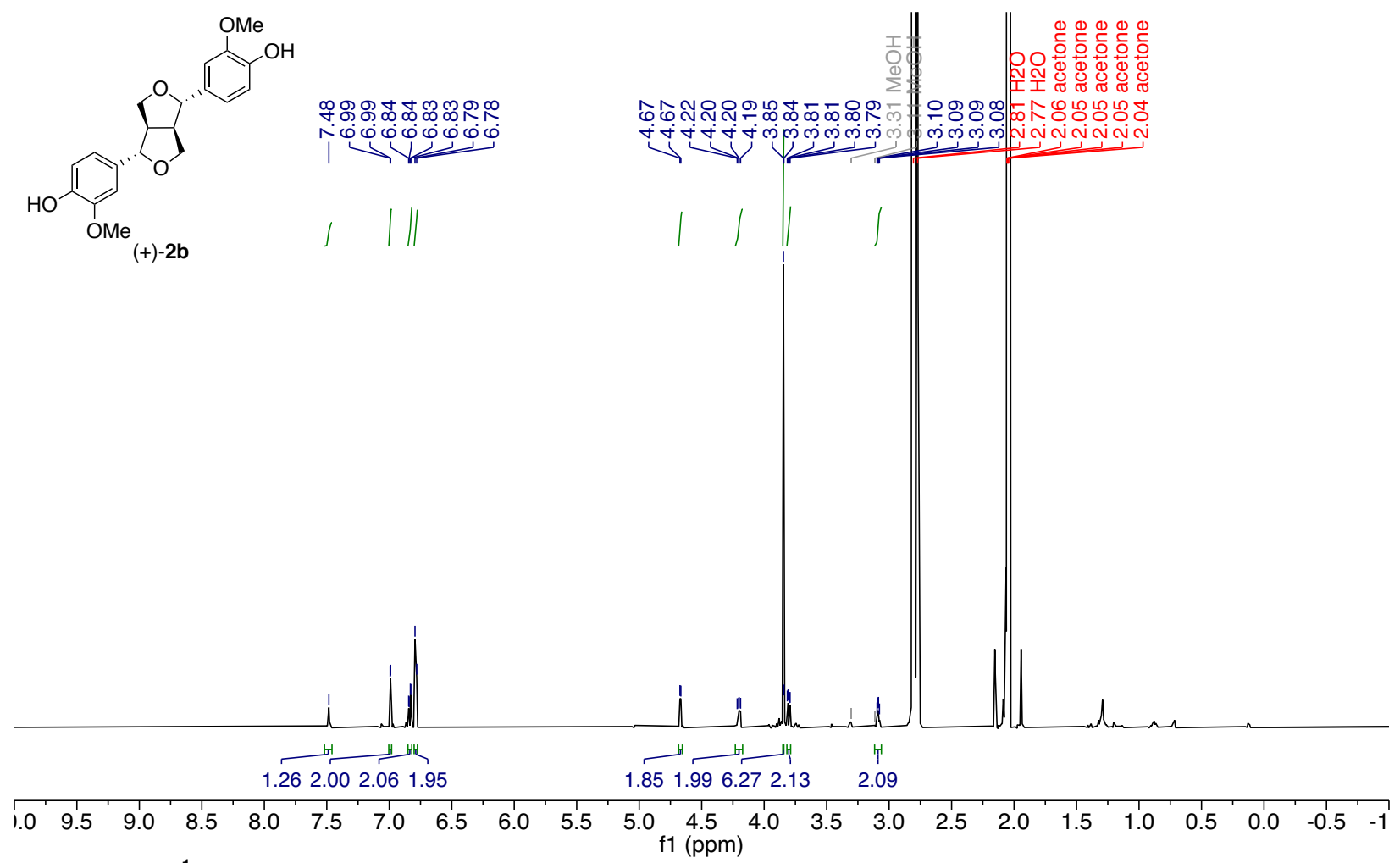

Figure S55. ${ }^{1} \mathrm{H}$ NMR spectrum of (+)-2b in acetone- $d_{6}$ 


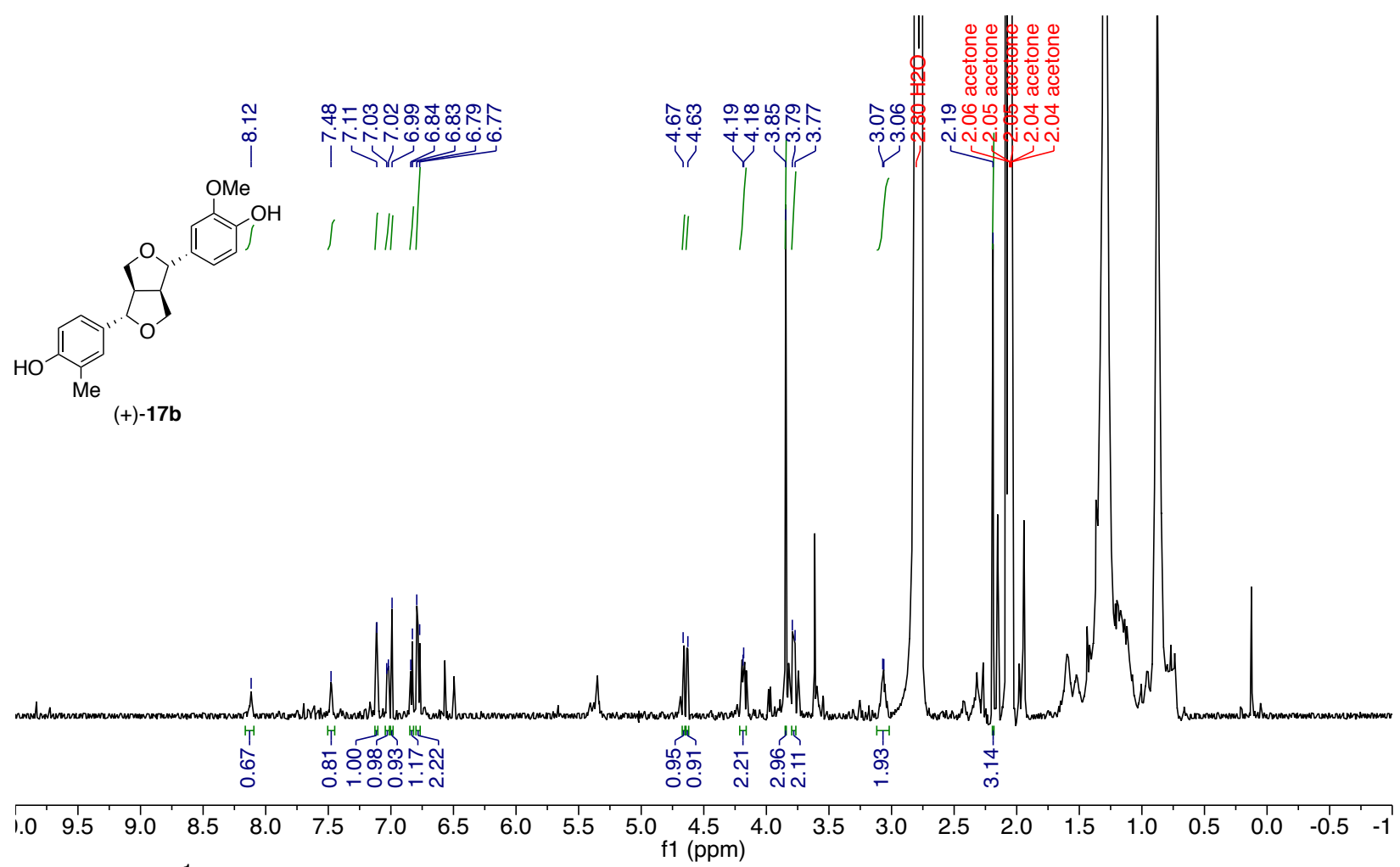

Figure S56. ${ }^{1} \mathrm{H}$ NMR spectrum of (+)-17b in acetone- $d_{6}$ 


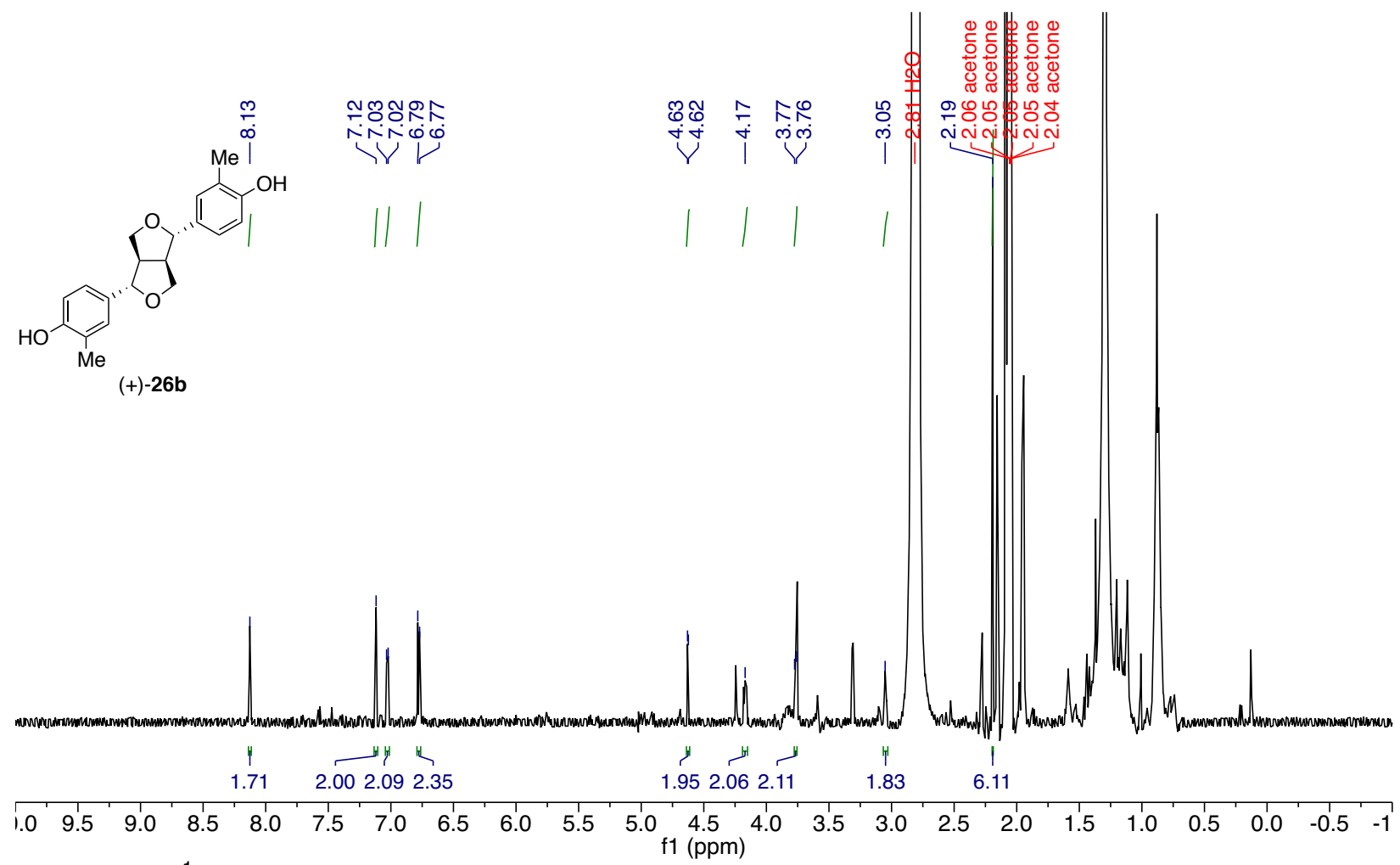

Figure S57. ${ }^{1} \mathrm{H}$ NMR spectrum of (+)-26b in acetone- $d_{6}$ 


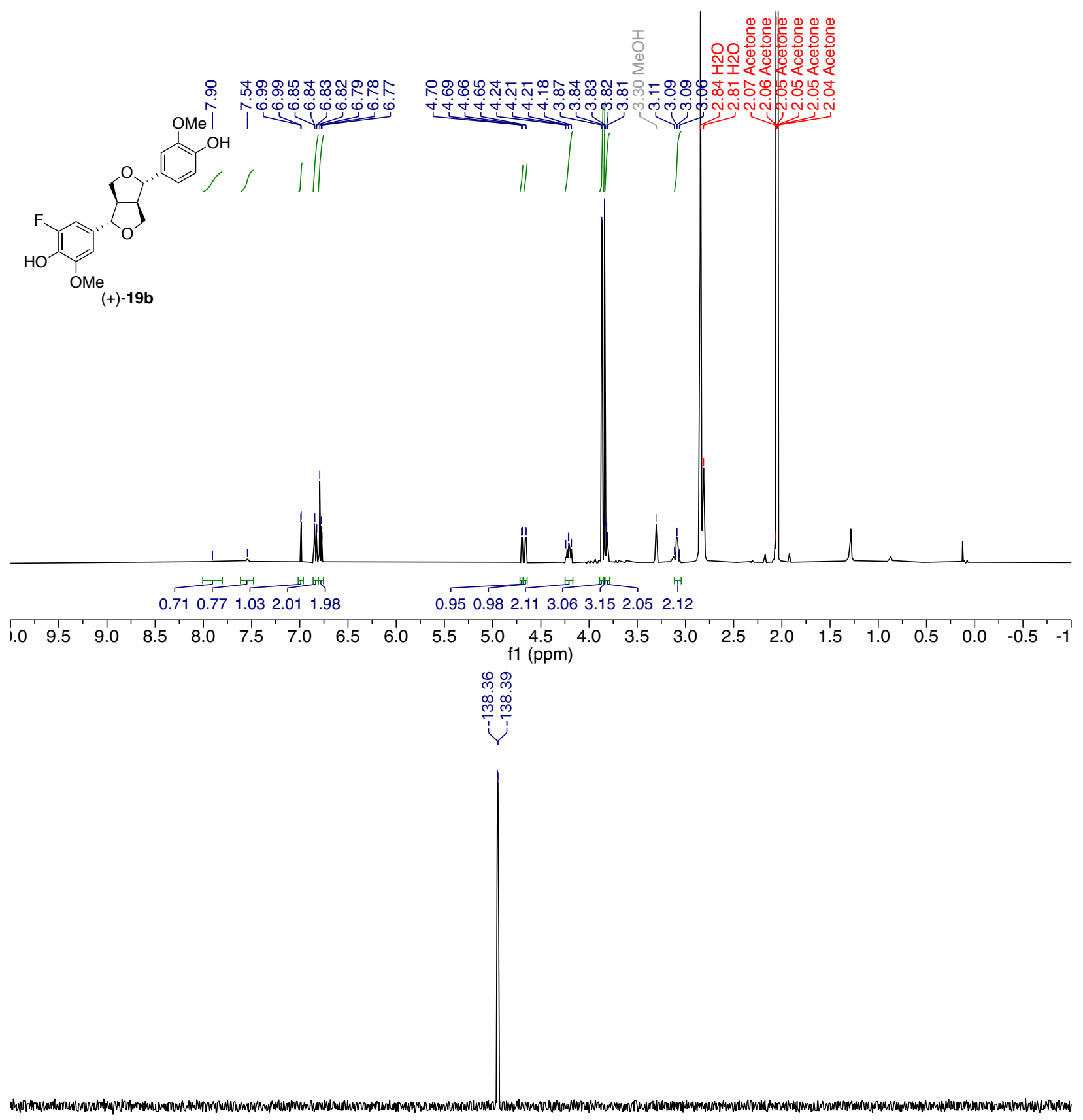

InW

\begin{tabular}{|c|c|c|c|c|c|c|c|c|}
\hline 20 & -125 & -130 & -135 & $\begin{array}{c}-140 \\
\mathrm{f} 1(\mathrm{ppm})\end{array}$ & -145 & -150 & -155 & -16 \\
\hline
\end{tabular}



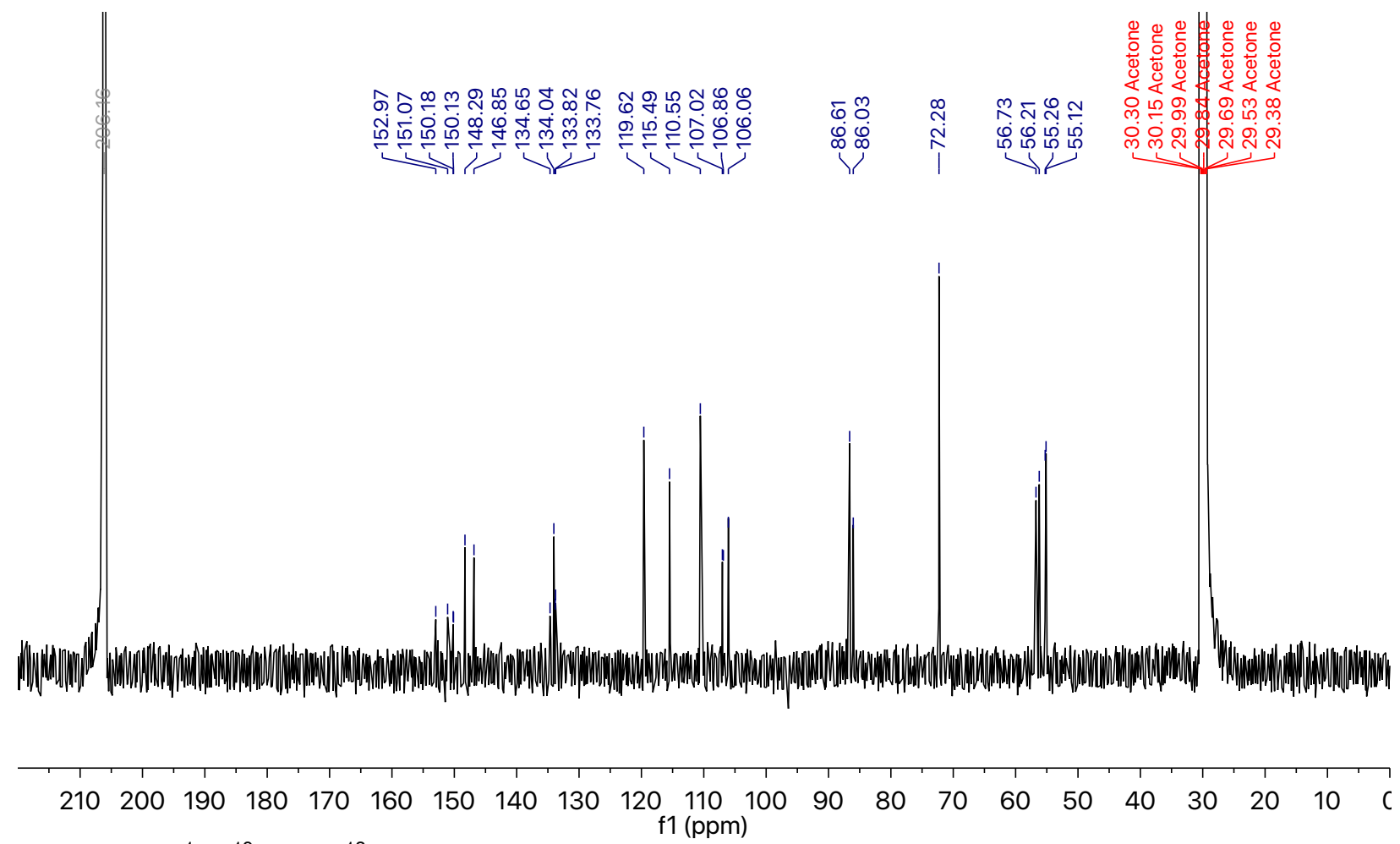

Figure S58. ${ }^{1} \mathrm{H},{ }^{19} \mathrm{~F}$, and ${ }^{13} \mathrm{C}$ NMR spectra of $(+)-19 \mathrm{~b}$ in acetone- $d_{6}$ 

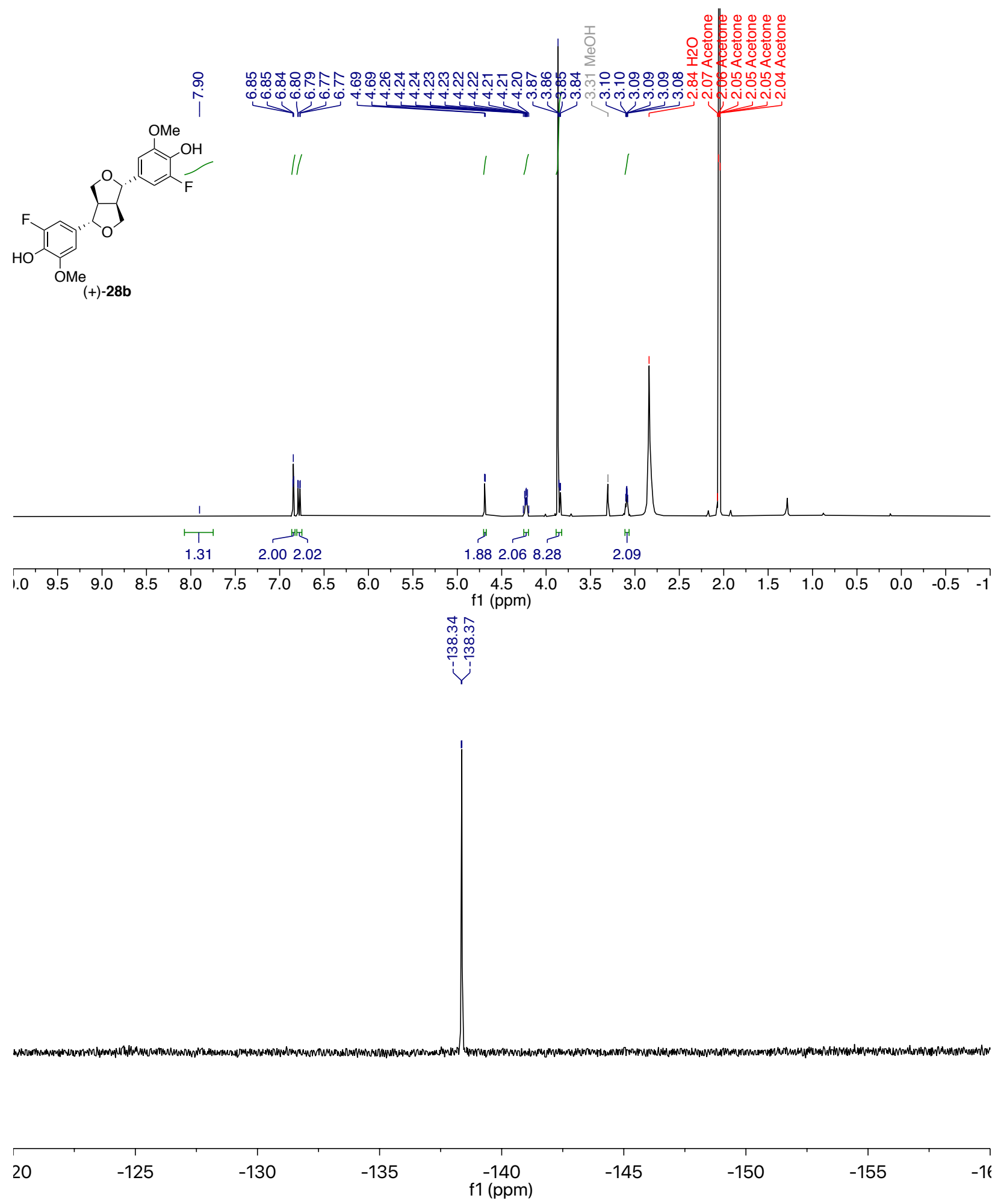

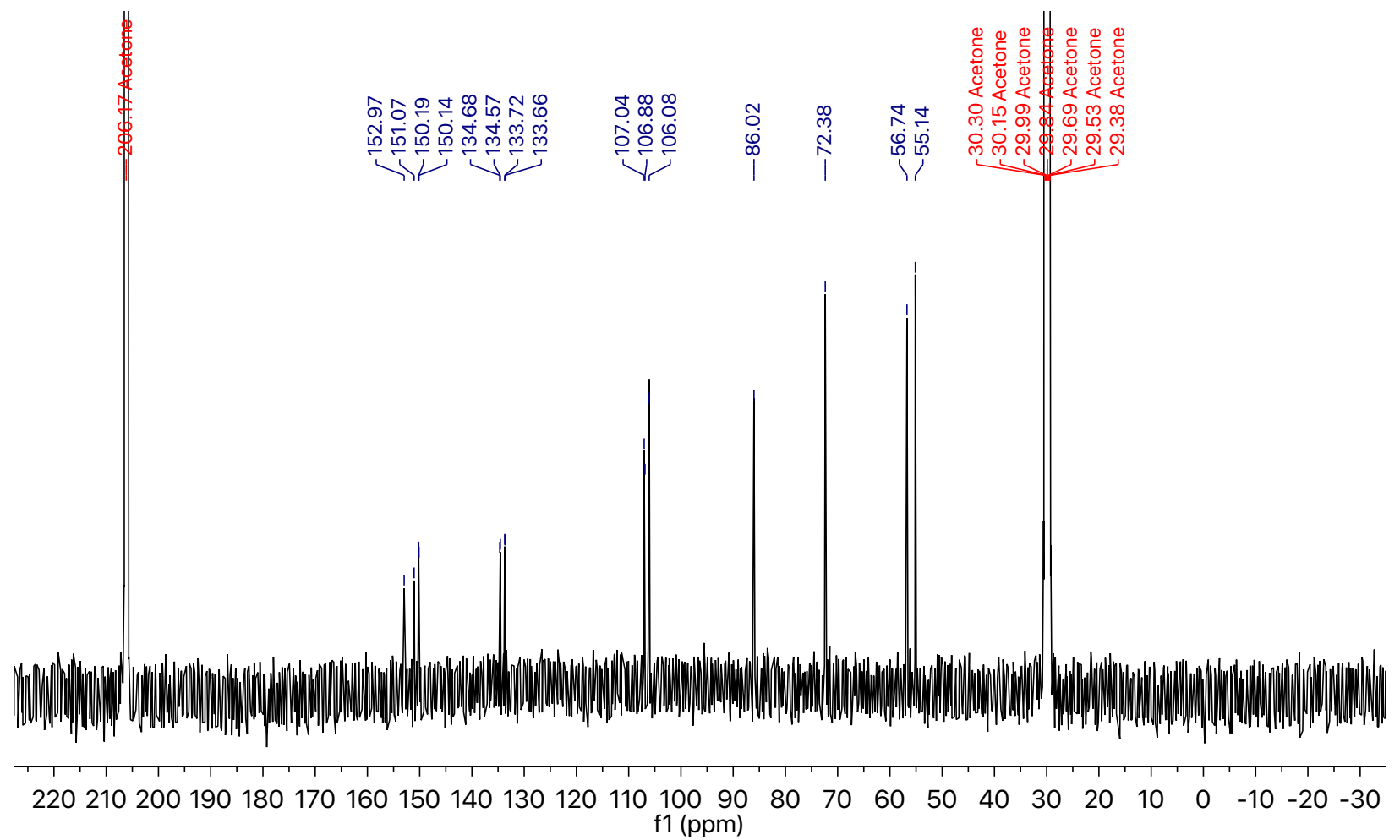

Figure S59. ${ }^{1} \mathrm{H},{ }^{19} \mathrm{~F}$, and ${ }^{13} \mathrm{C}$ NMR spectra of $(+)-\mathbf{2 8} \mathbf{b}$ in acetone- $d_{6}$ 

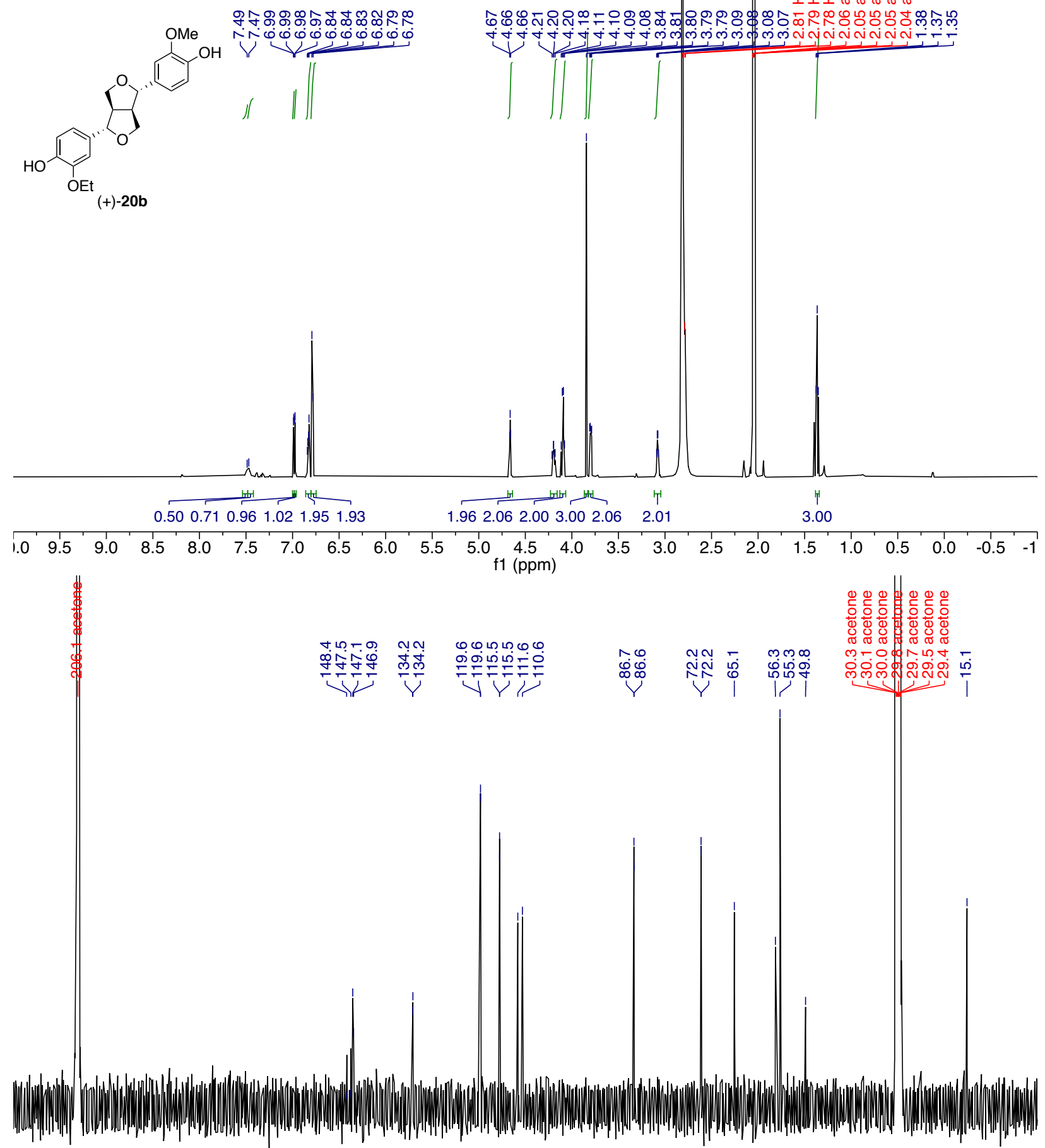

$\begin{array}{llllllllllllllllllllll}20 & 210 & 200 & 190 & 180 & 170 & 160 & 150 & 140 & 130 & 120 & 110 & 100 & 90 & 80 & 70 & 60 & 50 & 40 & 30 & 20 & 10\end{array}$

Figure S60. ${ }^{1} \mathrm{H}$ and ${ }^{13} \mathrm{C}$ NMR spectra of $(+)-20 \mathrm{~b}$ in acetone- $d_{6}$ 

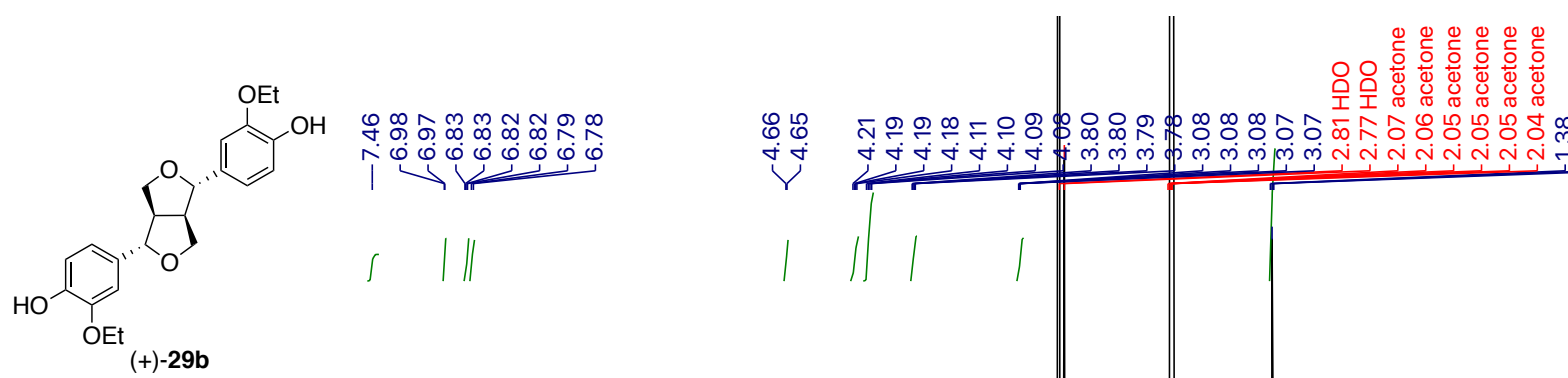

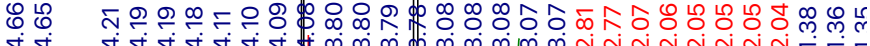

$+)-29 b$
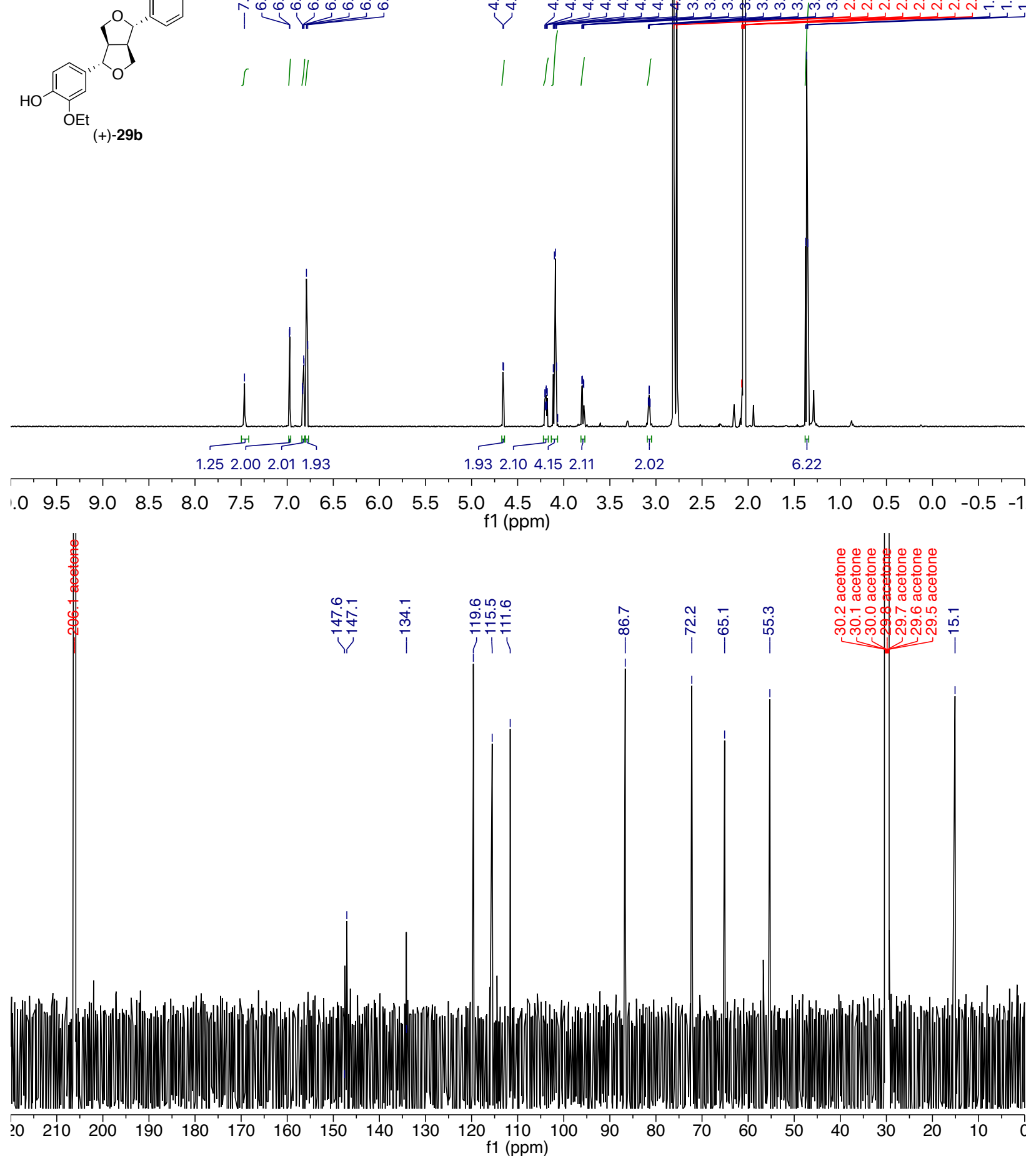

Figure S61. ${ }^{1} \mathrm{H}$ and ${ }^{13} \mathrm{C}$ NMR spectra of $(+)-29 \mathrm{~b}$ in acetone- $d_{6}$ 


\section{References}

1. Quideau, S.; Ralph, J., Facile Large-Scale Synthesis of Coniferyl, Sinapyl, and p-Coumaryl Alcohol. Journal of Agricultural and Food Chemistry 1992, 40 (7), 1108-1110.

2. Avó, J.; Cunha-Silva, L.; Lima, J. C.; Jorge Parola, A., Design and Synthesis of Photoactive lonic Liquids. Organic Letters 2014, 16 (10), 2582-2585.

3. Ramamoorthy, S. P.; Shen, Z.; Harrison, B. L. Tricyclic Compounds Useful as Serotonin Inhibitors and 5-HT1A Agonists and Antagonists. WO/2006/138549, 2006.

4. Romanov-Michailidis, F.; Viton, F.; Fumeaux, R.; Lévèques, A.; Actis-Goretta, L.; Rein, M.; Williamson, G.; Barron, D., Epicatechin B-Ring Conjugates: First Enantioselective Synthesis and Evidence for Their Occurrence in Human Biological Fluids. Organic Letters 2012, 14 (15), 3902-3905.

5. Kobayashi, T.; Taguchi, H.; Shigematsu, M.; Tanahashi, M., Substituent Effects of 3,5Disubstituted p-Coumaryl Alcohols on Their Oxidation Using Horseradish Peroxidase-H2O2 as the Oxidant. Journal of Wood Science 2005, 51 (6), 607-614.

6. $\quad$ Pickel, B.; Constantin, M.-A.; Pfannstiel, J.; Conrad, J.; Beifuss, U.; Schaller, A., An Enantiocomplementary Dirigent Protein for the Enantioselective Laccase-Catalyzed Oxidative Coupling of Phenols. Angewandte Chemie International Edition 2010, 49 (1), 202-204.

7. Wang, L. Q.; Zhao, Y. X.; Zhou, L.; Zhou, J., Lignans from Gnetum montanum Markgr. f. megalocarpua. Chemistry of Natural Compounds 2009, 45 (3), 424-426. 